\title{
Measurement of the Top Quark Pair Production Cross-Section in Dimuon Final States in Proton-Antiproton Collisions at $1.96 \mathrm{TeV}$
}

Dissertation zur Erlangung der Doktorwürde Vorgelegt von Jens-Peter Konrath

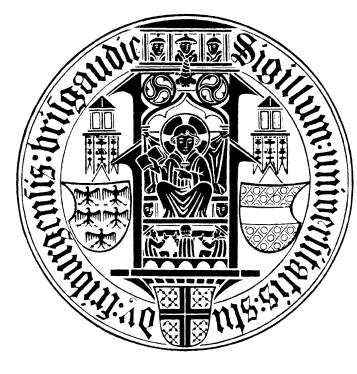


Dekan:

Prof. Dr. Kay Königsmann

Leiter der Arbeit:

Prof. Dr. Gregor Herten

Referent:

Prof. Dr. Gregor Herten

Koreferent:

Prof. Dr. Kay Königsmann

Datum der mündlichen Prüfung: 24. Oktober 2008 


\section{Contents}

\begin{tabular}{ll}
\hline 1. Introduction & 1
\end{tabular}

\begin{tabular}{ll}
\hline 2. Theoretical Aspects & 3
\end{tabular}

2.1. The Standard Model . . . . . . . . . . . . . . . . . . . . . . . . . . . . . . 3

2.1.1. Electroweak Interaction . . . . . . . . . . . . . . . . 4

2.1.2. The Higgs Mechanism . . . . . . . . . . . . . . . . . . . 6

2.1.3. Quantum Chromodynamics . . . . . . . . . . . . . . . . 7

2.2. Top Quark Production in Hadron Collisions . . . . . . . . . . . . . . . 7

2.2.1. Parton Distribution Functions . . . . . . . . . . . . . . . . . . 8

2.2.2. The Factorization Ansatz . . . . . . . . . . . . . . . . . . . 9

2.2.3. The Parton-Parton Cross Section for Top Quark Pair Production . 9

2.3. Top Quark Decay _ . . . . . . . . . . . . . . . . . . . . 15

2.4. Top Quark Spin Correlation . . . . . . . . . . . . . . . . . . . . . . . . . . 16

2.4.1. Polarized Top Quark Production . . . . . . . . . . . . . . . . . . 16

2.4.2. Constructing the Top Rest Frame . . . . . . . . . . . . . . . . . . . 20

2.4.3. Polarized Top Quark Decay . . . . . . . . . . . . . . . . . . . . . . 21

3. Experimental Apparatus 25

3.1. The Fermilab Accelerator Complex . . . . . . . . . . . . . . . . . . . . . . . . . . . . . . . 25

3.2. The DØDetector . . . . . . . . . . . . . . . . . . . . . 26

3.2.1. Tracking System . . . . . . . . . . . . . . . . . 26

3.2.2. Preshower Detectors . . . . . . . . . . . . . . . . . . . . . . . . . 29

3.2.3. Calorimeter System . . . . . . . . . . . . . . . . . . . . . . 29

3.2.4. Muon Spectrometer . . . . . . . . . . . . . . . . . . . . . . . . . . . . . . . . . . . . . . . . . . . 33

3.2.5. Luminosity Monitor . . . . . . . . . . . . . . . . . . . . . . 33

3.2.6. Forward Proton Detector . . . . . . . . . . . . . . . . . . . . . 34

3.2.7. Trigger and Data Acquisition . . . . . . . . . . . . . . 35

4. Event Reconstruction 39

4.1. Track Reconstruction . . . . . . . . . . . . . . . . . . . . . . . . . . . . . . 39

4.2. Vertexing . . . . . . . . . . . . . . . . . . . . . . . . 40

4.3. Muon Reconstruction and Identification . . . . . . . . . . . . . . . . . . 40

4.3.1. Muon Type and Quality . . . . . . . . . . . . . . . . . . . . . 40

4.3.2. Central Track Quality Definitions . . . . . . . . . . . . . . . . . . . 42

4.3.3. Muon Isolation . . . . . . . . . . . . . . . . . . . . . . . . . . . . . . . . . . . . . . . . 42

4.4. Electromagnetic Object Reconstruction . . . . . . . . . . . . . . . . . . . . 43

4.5. Jet Reconstruction and Identification . . . . . . . . . . . . . . . . . . . . . 44

4.5.1. Separation of Jets and Electromagnetic Objects . . . . . . . . . . 45

4.5.2. Jet Energy Scale Correction . . . . . . . . . . . . . . . . . . . . . . 45

4.6. Missing Transverse Energy $\left(E_{T}\right)$. . . . . . . . . . . . . . . . . . 50 
5. Data Sample and Monte Carlo Simulation 51

5.1. Data Sample. . . . . . . . . . . . . . . . . . . . . . . . . 51

5.1.1. Data Quality Criteria . . . . . . . . . . . . . . . . 51

$5.1 .2 . \quad$ Trigger Selection and Integrated Luminosity . . . . . . . . . . 52

5.2. Standard Model Monte Carlo Samples _ . . . . . . . . . . . . . . . . 55

5.2.1. Higher Order QCD corrections . . . . . . . . . . . . . . . 55

$5.2 .2 . \quad$ Event Generation $\ldots \ldots \ldots \ldots \ldots \ldots$

$5.2 .3 . \quad$ Modeling of Initial and Final State Radiation . . . . . . . . . 56

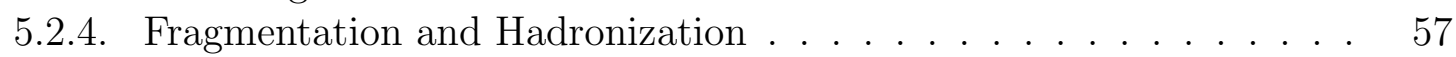

5.2 .5 . Detector Simulation and Event Reconstruction. . . . . . . . . . 57

5.2.6. Monte Carlo Corrections . . . . . . . . . . . . . . . . . . . 58

6. Measurement of the Top Quark Pair Production Cross Section in the Dimuon Final State $\quad 67$

6.1. Signal Monte Carlo . . . . . . . . . . . . . . . . . . . . . . . . . 67

6.2. Backgrounds . . . . . . . . . . . . . . . . . . . . 67

6.3. Dimuon Event Selection and Data/Monte Carlo Comparison . . . . . . . . 72

6.3.1. Object Identification . . . . . . . . . . . . . . . . . . . 72

6.3.2. Event Preselection and Corrections . . . . . . . . . . . . . 74

$6.3 .3 . \quad$ Background Rejection . . . . . . . . . . . . . . . . . 75

6.3.4. Missing Transverse Energy Significance . . . . . . . . . . . . . 75

6.3.5. Event Selection Summary and Cutflow . . . . . . . . . . . . . . 76

6.3.6. Fake Muon Background Estimation . . . . . . . . . . . . . . . . 90

6.4. Systematic Errors . . . . . . . . . . . . . . . . . . . . . . . . . 91

6.5. Results . . . . . . . . . . . . . . . . . . . . . . . . . . . . . 97

6.5.1. Combination with other Dilepton Cross Section Analyses . . . . . . 98

6.5.2. Determination of the Top Quark Mass from the Production Cross

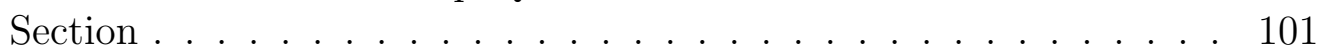

6.5.3. Charged Higgs Search . . . . . . . . . . . . . . 105

7. Monte Carlo Studies of the Top - Anti-Top Spin Correlation in the Dimuon Final State

7.1. Reconstruction of Dilepton Events . . . . . . . . . . . . . . . . . . . . . . . 109

7.1.1. Particle Level Corrections . . . . . . . . . . . . . . . . . . . . 109

7.1.2. Solving the Event Kinematics . . . . . . . . . . . . . . . . . . 113

7.2. Reweighting the PYTHIA Monte Carlo . . . . . . . . . . . . . . . . 114

7.3. Asymmetry Measurement . . . . . . . . . . . . . . . . . . . . . . 115

$\begin{array}{ll}\text { 8. Conclusion } & 125\end{array}$

\begin{tabular}{ll}
\hline 9. Acknowledgements & 127
\end{tabular}

A. Data - Monte Carlo Comparison in the Zero and Two Jet Inclusive Bin 129

B. $E_{\text {jet }}$ versus $E_{\text {parton }}$ Distributions and One Dimensional Profiles 137

C. Calibration Plots of the Spin Correlation Measurement for Various Input Scenarios and Ensemble Tests 
C.1. Calibration curves . . . . . . . . . . . . . . . . . . . . 143

C.2. Ensemble Tests . . . . . . . . . . . . . . . . . . 151

\begin{tabular}{ll}
\hline Bibliography & 166
\end{tabular} 


\section{List of Figures}

2.1. Parton densities for the up and down quark and the gluon in the proton according to CTEQ version 6.5 on the left hand side and MRST 2006 NNLO on the right hand side. $Q^{2}$ was set to $m_{t o p}^{2}=30625 \mathrm{GeV}^{2}$. . . . . . . . . 8

2.2. Top quark pair production through quark-antiquark annihilation . . . . . . 12

2.3. Top quark pair production through gluon fusion . . . . . . . . . . . . . . . 12

2.4. Dependence of the NLO-NLL ${ }^{\text {res }}$ and approx. NNLO top quark pair production cross-section on the top mass. The cross-section was parametrized as a polynomial of order 5 . The parameters can be found in [47]. . . . . . . . . 14

2.5. Top quark decay to a $b$ quark and $W^{+}$, which in turn decays to two light

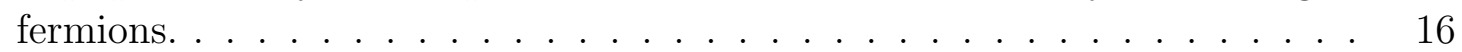

2.6. Spin configuration of top and antitop quark pairs produced via quarkantiquark-annihilation (left) and gluon fusion (right). For the quark-antiquarkannihilation, the likespin (top) and unlikespin (bottom) states of the ${ }^{3} S$ state

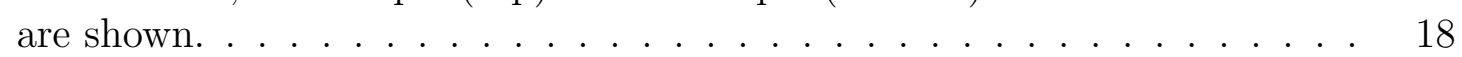

2.7. Spin alignment of top and antitop quark pairs at the production threshold (left) and well above (right). . . . . . . . . . . . . . . 18

2.8. Dependence of the spin analyzing power of the top quark decay products on the top quark mass. The analyzing power for the lepton and the down-type quark is constant for all top masses. . . . . . . . . . . . . . . . . 22

3.1. Map of the accelerator complex at FNAL (from Ref. [53]) . . . . . . . 26

3.2. Side view of the upgraded D $\varnothing$ detector (from Ref. [55]). . . . . . . . . 27

3.3. Schematic view of the DØ tracking system (from Ref. [55]). . . . . . . . . 28

3.4. The design of the Silicon Microstrip Tracker with its barrels and disks (from

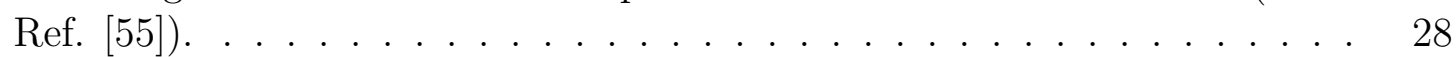

3.5. Isometric view of the calorimeter system (from Ref. [55]) . . . . . . . . 30

3.6. Schematic view of a portion of the DØ central end endcap calorimeter showing the segmentation pattern. The lines indicate pseudorapidity intervals from the center of the detector (from Ref. [55]). . . . . . . . . . . 31

3.7. Layout of the electromagnetic part of the endcap calorimeter. $M H$ indicates the middle hadronic section of the endcap. (from Ref. [55]). . . . . . . 32

3.8. Exploded view of the muon wire chambers (from Ref. [55]) . . . . . . . . . 34

3.9. Overview of the D $\varnothing$ trigger and data acquisition system (from Ref. [55]) . 35

3.10. Diagram of the Level 1 and Level 2 trigger systems. The data flow is indicated by the arrows. (from Ref. [55]) . . . . . . . . . . 36 ]

4.1. Muon identification efficiency in the $\eta_{d_{e t}}-\phi$ plane for muons with "loose" quality, as measured in data (taken from [59]). Muons identified in the "bottom hole" where the detector feet are, are rejected. . . . . . . . . . . . 41 
4.2. Efficiency for a "medium" track match as a function of $\eta_{C F T}$, as measured in data (taken from [59]). The efficiency is shown for for different ranges of track $\mathrm{z}$ values. . . . . . . . . . . . . . . . . . . . . . . . . . 42

4.3. Efficiency of muon isolation criteria versus $p_{T}$ measured in data. . . . . . . 43

4.4. Efficiency of muon isolation criteria versus $\eta$ measured in data. . . . . . . . 43

4.5. Estimated total jet offset energy (in $\mathrm{GeV}$ ) as a function of $\eta_{\text {jet }}^{\text {det }}$, for jets with $\mathcal{R}_{\text {cone }}=0.5$ [68]. The different lines show the prediction for noise and pile-up (NP) only $\left(n_{P V}=1\right)$, as well as NP and multiple interactions (MI;

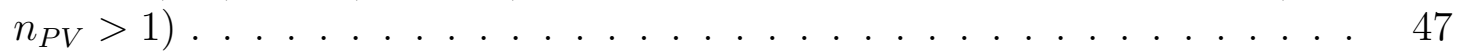

4.6. Examples of relative response correction derived with MPF method for jets in MC (top) and data (bottom) [68]. Shown are the corrections in $\eta_{\text {det }}<0.1$ (left), $0.2<\eta_{\text {det }}<0.3$ (middle) and $0.2<\eta_{\text {det }}<0.3$ (right) bins. . . . . . . 47

4.7. Absolute response correction derived with the MPF method for jets in MC

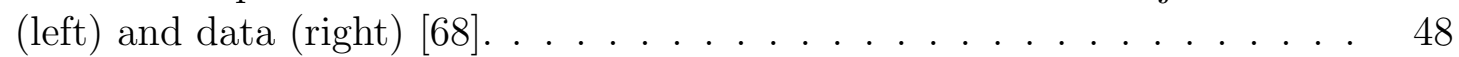

\begin{tabular}{llll}
\hline 4.8. Examples of the showering correction in MC (left) and data (right) [68] . . 48 & 48
\end{tabular}

4.9. Summary of all JES corrections as a function of uncorrected jet $p_{T}$ in MC (left) and data (right) [68] . . . . . . . . . . . . . . . . . . . . . . . . . 49

4.10. Summary of all JES corrections as a function of $\eta$ in MC (left) and data (right) for jets with an uncorrected transverse momentum of $50 \mathrm{GeV}$ [68].

5.1. Comparison of the instantaneous luminosity distribution in Run IIa data and the luminosity distribution of the overlaid zero bias event in $t t$ Monte Carlo before and after reweighting. The distributions were normalized to

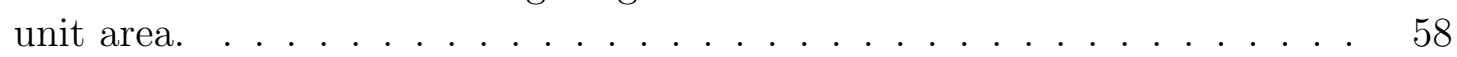

5.2. Relative muon identification efficiency correction [59] . . . . . . . . . . . . . 60

5.3. Relative track matching efficiency correction (as found in [59]). . . . . . . . 60

5.4. Comparison of the $Z$ peak in $Z \rightarrow \mu \bar{\mu}$ events in data and MC where both muons have SMT hits and $\left|\eta_{C F T}\right|<1.6$ (as found in [59]). In MC, the muon $p_{T}$ was smeared according to eq. 5.4 . . . . . . . . . . . . . 61

5.5. Comparison of data and smeared Monte Carlo for events where both muons have SMT hits and $\left|\eta_{C F T}\right|<1.6$ (as found in [59]). The two Monte Carlo distributions represent the $\pm 1 \sigma$ variation of the average smearing. The two data error bars represent different track qualities (medium and none). . . . 62

5.6. Efficiency of the logic OR of all single muon triggers in the triggerlists v13.2v13.99 (taken from [82]) for loose quality, medium track matched muons.

5.7. Distributions of $\Delta S$ for different ranges of $p_{T}^{\gamma}$ (taken from [83]). In the upper plots $\left(18<p_{T}^{\gamma}<23\right)$ fits by the product of an error function and a Gaussian are superimposed. The dashed curve corresponds to an extrapolation of the Gaussian part of the fit function in the region affected by the turn-on. In the bottom plots $\left(70<p_{T}^{\gamma}<75\right)$ the $\Delta S$ distribution is fitted by a Gaussian. The yellow bands indicate the statistical error. . . . . . . . . . . . . . 65

6.1. Top quark pair production (through quark-antiquark annihilation) and decay 67

$6.2 . W$ boson pair production and decay . . . . . . . . . . . . . 68

$6.3 . \quad W Z$ boson production and decay . . . . . . . . . . . . . . . . . . . . 68

$6.4 . \quad Z$ boson pair production and decay . . . . . . . . . . . . . . . . . . . . 68 
6.5. The $Z p_{T}$ distribution calculated from the muon pair momenta and jet multiplicity after $Z p_{T}$ reweighting. Points represent data. . . . . . . . . . 71

6.6. The $E_{T}$ significance distribution after cut $15 \mid$ of Section 6.3 .5 . . . . . . . . . 76

6.7. Distributions of $\mathbb{E}_{T}$ on the left and $\mathbb{E}_{T}$ significance on the right hand side illustrating the data and MC disagreement after the $E_{T}>\mathrm{GeV}$ cut. . . . . 79

6.8. Leading jet transverse momentum distribution. . . . . . . . . . . . . . . 84

6.9. Leading jet pseudo-rapidity distribution. . . . . . . . . . . . . . . . 84

6.10. Color code used in the plots shown on this page . . . . . . . . . . . . . 84

6.11. Next-to-leading jet transverse momentum distribution. . . . . . . . . . . . . 85

6.12. Next-to-leading jet pseudo-rapidity distribution. . . . . . . . . . . . . 85

6.13. Color code used in the plots shown on this page . . . . . . . . . . . . . 85

6.14. Leading muon transverse momentum distributions. . . . . . . . . . . . . . 86

6.15. Leading muon $\eta$ distributions. . . . . . . . . . . . . . . . . . . . . 86

6.16. Color code used in the plots shown on this page . . . . . . . . . . . . 86

6.17. Next-to-leading muon transverse momentum distributions. . . . . . . . . . 87

6.18. Next-to-leading muon $\eta$ distributions. . . . . . . . . . . . . . . 87

6.19. Color code used in the plots shown on this page . . . . . . . . . . . . . 87

6.20. Dimuon invariant mass distributions. . . . . . . . . . . . . . . . . . . . . . . . . . . . . . . . 88

6.21. $E_{T}$ distributions. . . . . . . . . . . . . . . . . 88

6.22. Color code used in the plots shown on this page . . . . . . . . . . . . 88

6.23. $E_{T}$ significance distributions. . . . . . . . . . . . . . . . 89

6.24. Color code used in the plots shown on this page . . . . . . . . . . . . . . 89

6.25. Dependence of the top quark pair production cross section on the top mass. 94

6.26. Dependence of the top quark pair production cross-section in the dimuon final state on the top mass. The single data point at $m_{t o p}=170 \mathrm{GeV}$ shows the measured value and its error. . . . . . . . . . . . . . . . . . 99

6.27. Dependence of the combined dilepton top quark pair production crosssection on the top mass. The single data point at $m_{t o p}=170 \mathrm{GeV}$ shows the combined cross-section and its error. . . . . . . . . . . . . . . . . . 102

6.28. The combined dilepton top quark production cross section overlaid with different theory predictions. The blue vertical lines indicate the uncertainty of $m_{t o p}$ induced by the theoretical uncertainty. The orange lines indicate the uncertainty of $m_{t o p}$ due to the combined statistical and systematic uncertainty of the cross-section measurement. . . . . . . . . . . . . . . . . 104

6.29. The branching ratio for top decays assuming that the only decay modes are $t \rightarrow W^{+} b$ and $t \rightarrow H^{+} b$ as calculated with CPsuperH [103] (as found in [7]). 105

6.30. The expected and observed limit on the branching ratio of $t \rightarrow H^{+} b \rightarrow \tau^{+} \nu b$ as a function of the charged Higgs mass in the tauonic model. The solid and dashed lines indicate predictions for $\operatorname{BR}\left(t \rightarrow H^{+} b\right)$ for various values of $\tan (\beta)$. The yellow band indicates the Feldman-Cousins $95 \%$ confidence level band. . . . . . . . . . . . . . . . . . . . . . . . 107

6.31. The observed and expected limit in the $m_{H^{+}}$vs. $\tan (\beta)$ plane. The hashed area indicates the region in this plane that is excluded by this search. . . . 107 
6.32. The expected and observed limit on the branching ratio of $t \rightarrow H^{+} b \rightarrow \tau^{+} \nu b$ as a function of the charged Higgs mass for simultaneous fit of $\mathrm{BR}(t \rightarrow$ $\left.H^{+} b\right)$ and $\sigma_{p \bar{p} \rightarrow t \bar{t}}$ in the tauonic model. The solid and dashed lines indicate predictions for $\mathrm{BR}\left(t \rightarrow H^{+} b\right)$ for various values of $\tan (\beta)$. The yellow band indicates the Feldman-Cousins $95 \%$ confidence level band. . . . . . . . . . 108

6.33. The observed and expected limit in the $m_{H^{+}}$vs. $\tan (\beta)$ plane derived with the simultaneous fit. The hashed area indicates the region in this plane that is excluded by this search. . . . . . . . . . . . . . . . . . 108

7.1. $E_{\text {jet }}$ versus $E_{\text {parton }}$ in the $0<|\eta|<0.5$ bin. . . . . . . . . . . . . . . 111

7.2. $\left\langle E_{\text {jet }}\right\rangle$ vs. $E_{\text {parton }}$ histogram derived from Fig. 7 7.1|| The solid line is the result of the fit in the shown region of $30 \mathrm{GeV}<\mathrm{E}_{\text {parton }}<400 \mathrm{GeV}$. . . . . 111

7.3. Invariant top mass distributions using the generated b quark, the reconstructed $b$ jet and the PLC corrected b jet. The Monte Carlo sample was generated with $m_{\text {top }}$ set to $170 \mathrm{GeV} . \ldots \ldots$. . . . . . . . . . . . 112

7.4. Examples of 2D $\cos \theta_{\ell}$ vs. $\cos \theta_{\bar{\ell}}$ distributions . . . . . . . . . . . . . . 115

7.5. Various $\cos \theta_{i} \times \cos \theta_{\bar{i}}$ distributions when $C_{\ell \bar{\ell}}$ is reweighed to one. Similar to the reweighting procedure, generated 4-momenta were employed to create the distributions. . . . . . . . . . . . . . . . . 117

7.6. Calibration curves derived by measuring the spin asymmetry at MC truth level for various $\left.C_{\ell \bar{\ell}}\right|_{g e n}$ values. . . . . . . . . . . . . . . . . . . . 118

7.7. Calibration plots for the measurement of $C_{\ell \bar{\ell}}$. The letters correspond to Tab. 7.2 indicating the type of inputs to the kinematic solver. (h) uses no MC truth information and hence resembles the measurement in data. . . . . . . 120

7.8. Calibration plots for the measurement of $C_{b \bar{b}}$. The letters correspond to Tab. 7.2 indicating the type of inputs to the kinematic solver. (h) uses no MC truth information and hence resembles the measurement in data. . . . 121

7.9. Ensemble tests for the measurement of $C_{\ell \bar{\ell}} .100$ ensembles with 8 events each were generated. The error bars in the calibration curve plots indicate

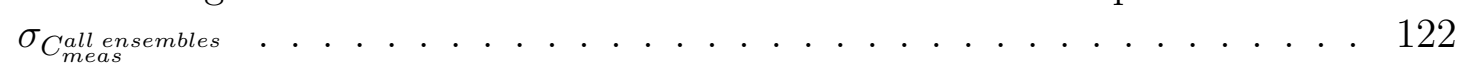

7.10. Ensemble tests for the measurement of $C_{b \bar{b}}$. 100 ensembles with 8 events each were generated. The error bars in the calibration curve plots indicate

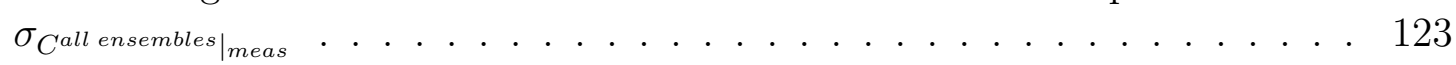

A.1. Leading jet transverse momentum distribution. . . . . . . . . . . . . . . . 130

A.2. Leading jet pseudo-rapidity distribution. . . . . . . . . . . . . . . . . . . . . . . . . . . . . 130

A.3. Color code used in the plots shown on this page . . . . . . . . . . . . . . . 130

A.4. Next to leading jet transverse momentum distribution. . . . . . . . . . . . 131

A.5. Next to leading jet pseudo-rapidity distribution. . . . . . . . . . . . . . . 131

A.6. Color code used in the plots shown on this page . . . . . . . . . . . . . . 131

A.7. Leading muon transverse momentum distributions. . . . . . . . . . . . . . 132

A.8. Leading muon $\eta$ distributions. . . . . . . . . . . . . . . . . . . . . . . . 132

A.9. Color code used in the plots shown on this page . . . . . . . . . . . . . . . 132

A.10.Next to leading muon transverse momentum distributions. . . . . . . . . . 133

A.11.Next to leading muon $\eta$ distributions. . . . . . . . . . . . . . . . . . 133

A.12.Color code used in the plots shown on this page . . . . . . . . . . . . . . . 133

A.13.Dimuon invariant mass distributions. . . . . . . . . . . . . . . . . . . . . . 134 


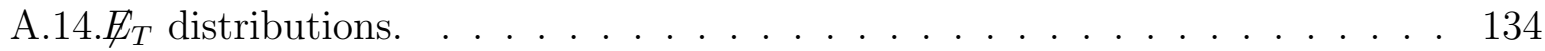

A.15.Color code used in the plots shown on this page . . . . . . . . . . . 134

A.16. $E_{T}$ significance distributions. . . . . . . . . . . . . . 135

A.17.Color code used in the plots shown on this page . . . . . . . . . . . 135

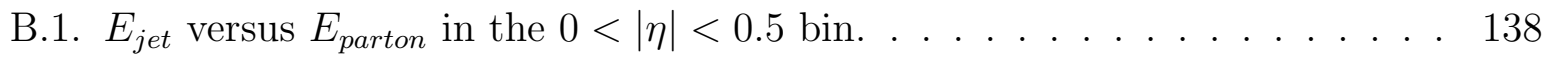

\begin{tabular}{|l|l||l|l|}
\hline B.2. $\left\langle E_{\text {jet }}\right\rangle$ vs. $E_{\text {parton }}$ histogram derived from Fig. & B.1 & The solid line is the
\end{tabular} result of the fit in the shown region of $30 \mathrm{GeV}<\mathrm{E}_{\text {parton }}<400 \mathrm{GeV} . \ldots 138$

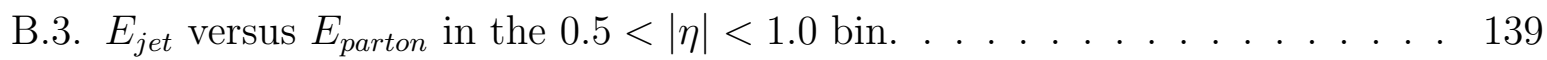

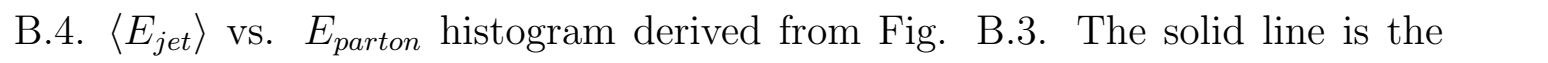
result of the fit in the shown region of $30 \mathrm{GeV}<\mathrm{E}_{\text {parton }}<400 \mathrm{GeV} . \quad \ldots \quad 139$

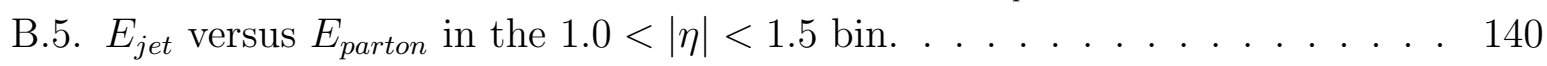

B.6. $\left\langle E_{\text {jet }}\right\rangle$ vs. $E_{\text {parton }}$ histogram derived from Fig. $\mid$ B. . result of the fit in the shown region of $30 \mathrm{GeV}<\mathrm{E}_{\text {parton }}<400 \mathrm{GeV} . \quad \ldots \quad 140$

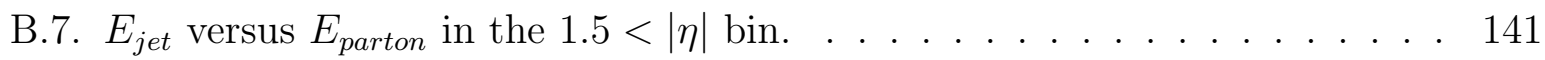

B.8. $\left\langle E_{\text {jet }}\right\rangle$ vs. $E_{\text {parton }}$ histogram derived from Fig. $\mid$ B.7| The solid line is the result of the fit in the shown region of $30 \mathrm{GeV}<\mathrm{E}_{\text {parton }}<400 \mathrm{GeV} . \quad \ldots \quad 141$

C.1. Calibration plots for the measurement of $C_{\ell b}$. The letters correspond to Tab.

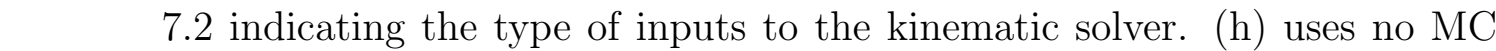
truth information and hence resembles the measurement in data. . . . . . . 144

C.2. Calibration plots for the measurement of $C_{\overline{\ell b}}$. The letters correspond to Tab. \begin{tabular}{|l|l|l|}
\hline 7.2 & indicating the type of inputs to the kinematic solver. (h) uses no MC \\
\hline
\end{tabular} truth information and hence resembles the measurement in data. . . . . . . 145

C.3. Calibration plots for the measurement of $C_{W^{+} W^{-}}$. The letters correspond to Tab. 7.2 indicating the type of inputs to the kinematic solver. (h) uses no MC truth information and hence resembles the measurement in data. . 146

C.4. Calibration plots for the measurement of $C_{W-\bar{\ell}}$. The letters correspond to Tab. 7.2 indicating the type of inputs to the kinematic solver. (h) uses no MC truth information and hence resembles the measurement in data. $\ldots$. . 147

C.5. Calibration plots for the measurement of $C_{W^{+\ell} \ell}$. The letters correspond to Tab. 7.2 indicating the type of inputs to the kinematic solver. (h) uses no MC truth information and hence resembles the measurement in data. . . . 148

C.6. Calibration plots for the measurement of $C_{W^{-} b}$. The letters correspond to Tab. 7.2 indicating the type of inputs to the kinematic solver. (h) uses no MC truth information and hence resembles the measurement in data. . . . 149

C.7. Calibration plots for the measurement of $C_{W+\bar{b}}$. The letters correspond to Tab. 7.2 indicating the type of inputs to the kinematic solver. (h) uses no MC truth information and hence resembles the measurement in data. . . . 150

C.8. Ensemble tests for the measurement of $C_{\ell b}$. 100 ensembles with 8 events each were generated. The error bars in the calibration curve plots indicate

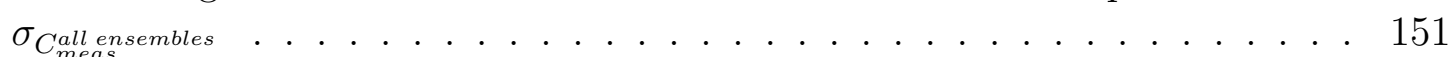

C.9. Ensemble tests for the measurement of $C_{\bar{b} \bar{\ell}}$. 100 ensembles with 8 events each were generated. The error bars in the calibration curve plots indicate

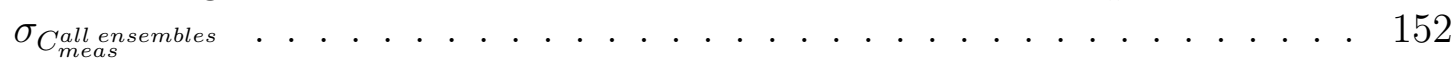


C.10.Ensemble tests for the measurement of $C_{W^{-} W^{+}} .100$ ensembles with 8 events each were generated. The error bars in the calibration curve plots indicate

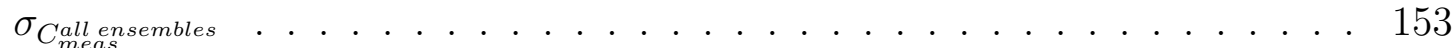

C.11.Ensemble tests for the measurement of $C_{W-\bar{\ell}} .100$ ensembles with 8 events each were generated. The error bars in the calibration curve plots indicate

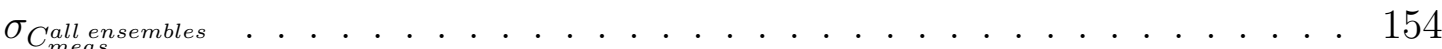

C.12.Ensemble tests for the measurement of $C_{W+\ell} .100$ ensembles with 8 events each were generated. The error bars in the calibration curve plots indicate

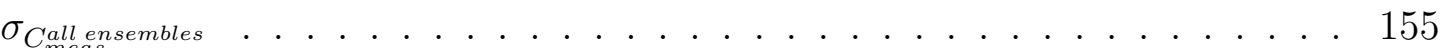

C.13.Ensemble tests for the measurement of $C_{W^{-} b}$. 100 ensembles with 8 events each were generated. The error bars in the calibration curve plots indicate

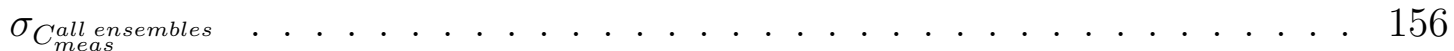

C.14.Ensemble tests for the measurement of $C_{W^{+} \bar{b}}$. 100 ensembles with 8 events each were generated. The error bars in the calibration curve plots indicate

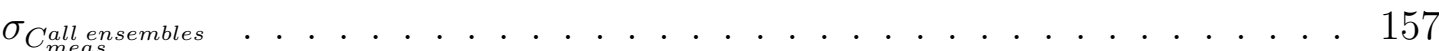




\section{List of Tables}

2.1. Overview of the Standard Model fermions and their masses [18]. . . . . . . 3

2.2. Overview of the Standard Model bosons and their masses. . . . . . . . . . 4

2.3. Weak isospin, hypercharge and charge of the left- and right-handed Standard

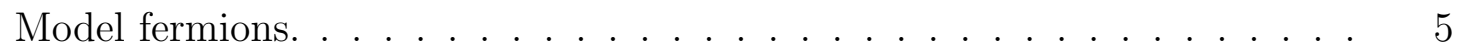

2.4. Comparison of different theory predictions found in the literature. $r_{\mu}$ denotes the variation of the renormalization and factorization scale, i.e. $\mu_{r}=\mu_{f}=r_{\mu} \times$

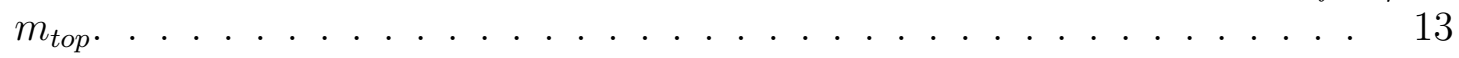

2.5. Branching fractions of the $t \bar{t}$ event signature categories as calculated with the $W$ boson decay widths found in [18], $\Gamma\left(W \rightarrow e \nu_{e}\right)=0.1075, \Gamma(W \rightarrow$ $\left.\mu \nu_{\mu}\right)=0.1057, \Gamma\left(W \rightarrow \tau \nu_{\tau}\right)=0.1125$ and $\left.\Gamma(W \rightarrow q \bar{q})=0.676.\right] . . . . \quad 16$

2.6. Analyzing power $\alpha$ for decay products of both semileptonic and hadronic top quark decays as a function of $\xi=m_{t}^{2} / m_{W}^{2} . \ldots \ldots 23$

5.1. Integrated luminosity collected with the OR of all single muon triggers and the trigger list version. . . . . . . . . . . . . . . 52

5.2. List of all single muon triggers available in the single muon trigger OR by triggerlist version. The trigger terms are explained in Tab. $5.3 . \mid \ldots . . .253$

5.3. Description of the trigger names. $p_{T}^{\text {local }}$ indicates that the muon momentum is measured in the muon detector. The isolation variables are calculated as the scalar sum of the energy or $p_{T}$ in a hollow cone with the given inner and outer radius in calorimeter or tracker, respectively. . . . . . . . . . . . . 54

6.1. LO and NLO (Diboson)/NNLO $(Z)$ cross sections for the various background processes [86], [87]. The K-factor of 1.33 for $Z$ production is derived in the central mass bin using the LO ALPGEN cross-section and the NNLO

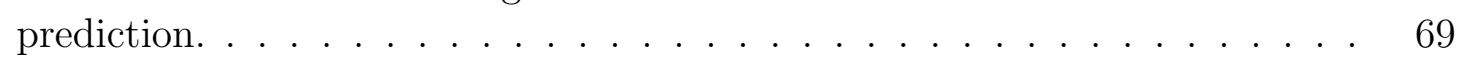

6.2. The number of events in ALPGEN $Z \rightarrow \mu \bar{\mu}$ MC samples before and after heavy flavor skimming, and their respective cross sections. See Sec. 5.2.2 for the explanation of $\mathrm{n} l p$, exclusive and inclusive. . . . . . . . . . . . . 70

6.3. The number of events in ALPGEN $Z \rightarrow \tau \bar{\tau}$ MC samples before and after heavy flavor skimming, and their respective cross sections. See Sec. 5.2 .2 for the explanation of $\mathrm{n} l p$, exclusive and inclusive. . . . . . . . . . . . . 72

6.4. Overview of the different muon types used in this analysis. . . . . . . . . . 73

6.5. Overview of the different electron types used in this analysis. . . . . . . . . 74

6.6. Selection efficiencies in $t \bar{t} \rightarrow \mu \bar{\mu}$ process. The numbers in parenthesis refer $\begin{array}{ll}\text { to the cut number in Section } \mid 6.3 .5 \text {. The quoted errors are statistical only. . } & 80\end{array}$

6.7. The measured and predicted event yield in $t \bar{t} \rightarrow \mu \bar{\mu}$ process. . . . . . . . 81

6.8. Event yield for the different $Z \rightarrow \mu \bar{\mu}$ samples. . . . . . . . . . . . . . . . 82

6.9 . Event yield for the different $Z \rightarrow \tau \bar{\tau}$ samples. . . . . . . . . . . . . . . . . . . 82

6.10. Event yield for the diboson samples. . . . . . . . . . . . . . . . . 82 
6.11. Selection efficiencies and related measured cross sections in $t \bar{t} \rightarrow \mu \bar{\mu}$ process for different top masses. The uncertainty on the selection efficiency are statistical only; The cross-section uncertainty shown includes both statistical and systematic ones. The cross-sections were extracted following the method in Sec. 6.5] . . . . . . . . . . . . . . . . . . . . . . . . 83

6.12. Vertex and opposite muon charge selection efficiencies in data and $t \bar{t} \mathrm{MC}$. The quoted errors are statistical only. . . . . . . . . . . . . . . . 83

6.13. The measured fake rates and signal efficiencies. All errors are statistical ones. 91

6.14. Systematic uncertainties by source and Monte-Carlo sample. Not shown is the statistic uncertainty on the QCD background, which is $102.53 \%$. No other sources contribute to the systematic uncertainty on the QCD back-

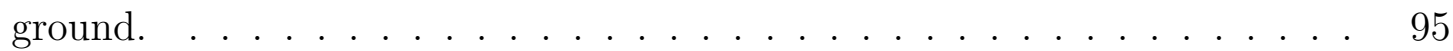

6.15. Summary of all sources of systematic uncertainties. . . . . . . . . . . . . 97

6.16. Expected background, observed and expected signal yield for $t \bar{t} \rightarrow \mu \mu$ channel. 98

7.1. Summary of the PLC parameters. The reconstructed jet energy $E_{\text {jet }}$ is

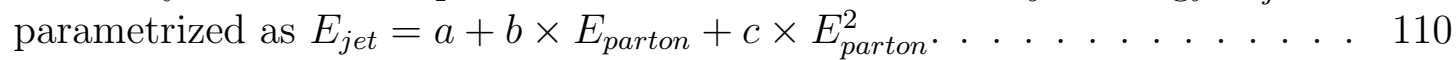

7.2. The efficiency to find at least one solution, the ratio of events with exactly two solutions to the number of events with at least one solution, the average number and RMS of solutions when at least one solution was found for different input scenarios. . . . . . . . . . . . . . . . . . . . . . 114 


\section{Introduction}

Particle physics deals with the fundamental building blocks of matter and their interactions. The vast number of subatomic particles can be reduced to twelve fundamental fermions, which interact by the exchange of spin-1 particles as described in the Standard Model (SM) of particle physics. The SM provides the best description of the subatomic world to date, despite the fact it does not include gravitation.

Following the relation $\lambda=h / p$, where $h$ is Planck's constant, for the examination of physics at subatomic scales with size $\lambda$ probes with high momenta $p$ are necessary. These high energies are accessible through particle colliders. Here, particles are accelerated and brought to collision at interaction points at which detectors are installed to record these particle collisions.

Until the anticipated start-up of the Large Hadron Collider at CERN, the Tevatron collider at Fermilab near Chicago is the highest energy collider operating in the world, colliding protons and anti-protons at a center-of-mass energy of $\sqrt{s}=1.96 \mathrm{TeV}$. Its two interaction points are covered by the multi purpose particle detectors D $\varnothing$ and CDF.

During the first data-taking period, known as Run I, the Tevatron operated at a centerof-mass energy of $1.8 \mathrm{TeV}$. This run period lasted from 1992 to 1996. During this period, the long-predicted top quark was discovered. From 1996 and 2001, the accelerator was upgraded to deliver higher instantaneous luminosities at its current center-of-mass energy. At the same time, the experiments were upgraded to take full advantage of the upgraded accelerator complex.

The Tevatron is currently the only accelerator in the world with a sufficient energy to produce top quarks. Studying top quark production, decay and properties is an important part of the D $\varnothing$ and CDF physics programs. Because of its large mass, the top quark is a unique probe of the Standard Model, and an interesting environment to search for new physics.

In this thesis, a measurement of the production cross-section of top quark pairs decaying to two muons is presented 1 . In addition, a Monte Carlo study of the top quark spin correlation measurement was carried out. This thesis is laid out as follows: chapter two gives a short overview over the Standard Model of particle physics and the theoretical aspects of unpolarized and polarized top quark production and decay, chapter three describes the accelerator complex and the DØ experiment whose data is used in this analysis. The Reconstruction of events recorded with the $\mathrm{D} \varnothing$ detector is explained in chapter four and the data and Monte Carlo samples used are presented in chapter five. Finally, the cross-section measurement is described in chapter six and the Monte Carlo study of top quark spin correlations in chapter seven.

\footnotetext{
${ }^{1}$ Preliminary results of this analysis have been reported in [1], 2], 3], 4], 5], 6], 7]. The final crosssection publication is in preparation.
} 


\section{Theoretical Aspects}

The first part of the following chapter gives an overview of the Standard Model $(S M)$ of particle physics. The second part describes in more detail the physics of top quarks within the SM. Throughout this thesis, we will set $\hbar=c=1$.

\subsection{The Standard Model}

The Standard Model [8] [9] [10] [11] [12] [13] [14] [15] [16] [17] sums up our current understanding of particle physics gathered over the last 40 years.

The particle content of the SM is divided into two groups according to their spin $s$ : bosons with spin 1 and fermions with spin $1 / 2$. An overview of the fundamental fermions is given in Table 2.1. Their interactions as described by the Standard Model are mediated by gauge bosons. An overview of the different gauge bosons is given in Table 2.2. There are four different known interactions: strong, weak, electromagnetic and gravitational. All but the latter are described through local gauge symmetries within the Standard Model, whereas gravitation is described by general relativity which is not part of the SM.

Fermions are divided further into two categories, leptons and quarks. Leptons only participate in the electroweak interactions, while quarks participate both in electroweak and strong interactions. Both leptons and quarks are divided into three generations. The first generation contains the electron (e), the electron-neutrino $\left(\nu_{e}\right)$ and the up- and down quark $(\mathrm{u}, \mathrm{d})$. The second generation contains the muon $(\mu)$, its neutrino $\left(\nu_{\mu}\right)$ and the charmand strange quark $(\mathrm{c}, \mathrm{s})$. Finally, the third generation consist of the tau $(\tau)$, the tau-neutrino $\left(\nu_{\tau}\right)$ and the bottom- and top quark $(\mathrm{b}, \mathrm{t})$. For each fermion exists an antifermion with identical mass, but opposite multiplicative quantum numbers. All but the lightest quarks and charged leptons are unstable and decay weakly into lighter fermions.

\begin{tabular}{|c||c|c||c|c|}
\hline \multicolumn{1}{|c||}{ Generation } & \multicolumn{2}{c||}{ Leptons } & \multicolumn{2}{c|}{ Quarks } \\
& Symbol & Mass $[\mathrm{MeV}]$ & Symbol & Mass $[\mathrm{MeV}]$ \\
\hline \hline \multirow{2}{*}{1 st } & $e^{-}$ & 0.511 & $\mathrm{~d}$ & 4 to 8 \\
& $\nu_{e}$ & $<3 \times 10^{-6}$ & $\mathrm{u}$ & 1.5 to 4 \\
\hline 2nd & $\mu^{-}$ & 105.7 & $\mathrm{~s}$ & 80 to 130 \\
& $\nu_{\mu}$ & $<2 \times 10^{-4}$ & $\mathrm{c}$ & $(1.15$ to 1.35$) \times 10^{3}$ \\
\hline 3rd & $\tau^{-}$ & 1777 & $\mathrm{~b}$ & $(4.6$ to 4.9$) \times 10^{3}$ \\
& $\nu_{\tau}$ & $<18.2$ & $\mathrm{t}$ & $172.4 \times 10^{3}$ \\
\hline
\end{tabular}

Table 2.1.: Overview of the Standard Model fermions and their masses [18]. 
Due to the small masses of the SM fermions and bosons, gravitation does not play a role at the current accessible energy scale. Quantum field theory $(Q F T)$ is the theoretical framework of the Standard Model, in which the gauge bosons are the quanta of the fields introduced to preserve local gauge symmetry. The gauge bosons mediate the interactions between the fermions.

\begin{tabular}{|c|c|c|c|c|}
\hline Name & Field & Interaction & Mass $[\mathrm{GeV}]$ & Charge [e] \\
\hline \hline Photon $\gamma$ & $A_{\mu}$ & electromagnetic & $<6 \times 10^{-26}$ & $<5 \times 10^{-30}$ \\
$Z$ Boson & $Z_{\mu}$ & electroweak & 91.19 & 0 \\
$W$ Bosons $W^{ \pm}$ & $W_{\mu}^{ \pm}$ & electroweak & 80.43 & \pm 1 \\
Gluon $g$ & $G_{\mu}^{a}$ & strong & 0 & 0 \\
\hline
\end{tabular}

Table 2.2.: Overview of the Standard Model bosons and their masses.

The Standard Model is a gauge theory based on the $S U(3)_{C} \times S U(2)_{L} \times U(1)_{Y}$ gauge group. The subgroup $S U(2)_{L} \times U(1)_{Y}$ represents the unified electromagnetic and weak interactions and is generated by the hypercharge $\mathrm{Y}$ and the weak isospin $\mathbf{T}$. The charge $\mathrm{Q}$ is then given by $T_{3}+Y / 2 . S U(3)_{C}$ is the gauge group of the strong interaction.

\subsubsection{Electroweak Interaction}

In quantum field theory quarks and lepton are represented by spinor fields $\Psi(x)$ which are functions of the continuous space-time coordinates $x_{\mu}$. As the weak interaction only couples to left-handed particles, it is necessary to introduce left- and right-handed fields through the projection operators $P_{L}=\frac{1}{2}\left(1-\gamma_{5}\right)$ and $P_{R}=\frac{1}{2}\left(1+\gamma_{5}\right)$,

$$
\begin{aligned}
& \Psi_{L}(x)=P_{L} \Psi(x) \text { and } \\
& \Psi_{R}(x)=P_{R} \Psi(x)
\end{aligned}
$$

The left-handed states of one generation are grouped into weak isospin doublets, the righthanded states form singlets. The isospin, hypercharge and charge assignments of the isospin doublets and singlets are shown in Table 2.3, assuming massless and therefore only left-handed neutrinos.

The Lagrangian of the electromagnetic and weak interactions can be derived by demanding that the free particle Lagrangian density

$$
\begin{aligned}
\mathcal{L}_{0} & =i \bar{\Psi} \gamma^{\mu} \partial_{\mu} \Psi \\
& =i \overline{\Psi_{L}(x)} \gamma^{\mu} \partial_{\mu} \Psi_{L}(x)+i \overline{\Psi_{R}(x)} \gamma^{\mu} \partial_{\mu} \Psi_{R}(x)
\end{aligned}
$$

be invariant under local phase transformations [12]:

$$
\Psi_{L} \longrightarrow e^{i g \alpha(x) \cdot \mathbf{T}+i g \prime \beta(x) Y} \Psi_{L} \quad \text { and } \quad \Psi_{R} \longrightarrow e^{i g \prime \beta(x) Y} \Psi_{R}
$$




\begin{tabular}{|c|c|c|c|c|c|c|c|}
\hline & 1st Generation & 2nd Generation & 3rd Generation & $\mathbf{T}$ & $T_{3}$ & $\mathrm{Y}$ & $\mathrm{Q}$ \\
\hline \hline \multirow{3}{*}{ Leptons } & $\left(\begin{array}{c}\nu_{e} \\
e\end{array}\right)_{L}$ & $\left(\begin{array}{c}\nu_{\mu} \\
\mu\end{array}\right)_{L}$ & $\left(\begin{array}{c}\nu_{\tau} \\
\tau\end{array}\right)_{L}$ & $\frac{1}{2}$ & $\frac{1}{2}$ & -1 & 0 \\
& $e_{R}$ & $\mu_{R}$ & $-\frac{1}{2}$ & -1 & -1 \\
& $\left(\begin{array}{c}u \\
\text { Quarks }\end{array}\right.$ & $\left(\begin{array}{c}c \\
d_{2}\end{array}\right)_{L}$ & $\left(\begin{array}{c}t \\
s_{2}\end{array}\right)_{L}$ & 0 & 0 & -2 & -1 \\
& $u_{R}$ & $c_{R}$ & $t_{R}$ & $\frac{1}{2}$ & $-\frac{1}{3}$ & $\frac{2}{3}$ \\
& $d_{R}$ & $s_{R}$ & $b_{R}$ & 0 & 0 & $-\frac{1}{3}$ & $-\frac{1}{3}$ \\
& $d_{R}$ & 0 & 0 & $-\frac{2}{3}$ & $-\frac{1}{3}$ \\
\hline
\end{tabular}

Table 2.3.: Weak isospin, hypercharge and charge of the left- and right-handed Standard Model fermions.

Here, the parameter $\alpha(x)$ is an arbitrary three-component vector and $\mathbf{T}=\left(T_{1}, T_{2}, T_{3}\right)$ is the weak isospin operator whose components are the generators of $\mathrm{SU}(2) . \quad \beta(x)$ is a one-dimensional function of $\mathrm{x}$ and $\mathrm{g}$ and g' are the coupling constants of the weak and electromagnetic force, respectively.

Demanding the Lagrangian $\mathcal{L}_{0}$ to be invariant requires the addition of terms to the free Lagrangian which involve four additional vector ( spin 1) fields, the isotriplet $\mathbf{W}_{\mu}=$ $\left(W_{1 \mu}, W_{2 \mu}, W_{3 \mu}\right)$ for $S U(2)_{L}$ and the singlet $\mathrm{B}_{\mu}$ for $U(1)_{Y}$. This is done by replacing the derivative $\partial_{\mu}$ in $\mathcal{L}_{0}$ by the covariant derivative

$$
D_{\mu}=\partial_{\mu}+i g \mathbf{W}_{\mu} \cdot \mathbf{T}+i g \prime \frac{1}{2} B_{\mu} Y
$$

and adding the kinetic terms of the gauge fields:

$$
\left[D_{\mu}, D_{\nu}\right]\left[D^{\mu}, D^{\nu}\right]=-\frac{1}{4} \mathbf{W}_{\mu \nu} \cdot \mathbf{W}^{\mu \nu}-\frac{1}{4} B_{\mu \nu} \cdot B^{\mu \nu}
$$

where $[\mathrm{A}, \mathrm{B}]$ is the usual commutator and

$$
\mathbf{W}_{\mu \nu}=\partial_{\mu} \mathbf{W}_{\nu}-\partial_{\nu} \mathbf{W}_{\mu}-g \cdot \mathbf{W}_{\mu} \times \mathbf{W}_{\nu} \quad B_{\mu \nu}=\partial_{\mu} B_{\nu}-\partial_{\nu} B_{\mu}
$$

For an electron-neutrino pair, for example, the resulting Lagrangian reads [12]:

$$
\begin{gathered}
\mathcal{L}_{\text {leptons }}=i \overline{\left(\begin{array}{c}
\nu_{e} \\
e
\end{array}\right)_{L}} \gamma^{\mu}\left[\partial_{\mu}+i g \mathbf{W}_{\mu} \cdot \mathbf{T}+i g \prime \frac{1}{2} B_{\mu} Y_{L}\right]\left(\begin{array}{c}
\nu_{e} \\
e
\end{array}\right)_{L}+ \\
+i \bar{e}_{R}\left[\partial_{\mu}+i g \prime \frac{1}{2} B_{\mu} Y_{R}\right] e_{R}+-\frac{1}{4} \mathbf{W}_{\mu \nu} \cdot \mathbf{W}^{\mu \nu}-\frac{1}{4} B_{\mu \nu} \cdot B^{\mu \nu}
\end{gathered}
$$

again assuming massless neutrinos. This can be recast more clearly as

$$
\begin{aligned}
& \mathcal{L}_{\text {leptons }}=i \overline{\left(\begin{array}{c}
\nu_{e} \\
e
\end{array}\right)_{L}} \gamma^{\mu} \partial_{\mu}\left(\begin{array}{c}
\nu_{e} \\
e
\end{array}\right)_{L}+i \bar{e}_{R} \gamma_{\mu} \partial_{\mu} e_{R}+i \overline{\left(\begin{array}{c}
\nu_{e} \\
e
\end{array}\right)_{L}}\left[i g \mathbf{W}_{\mu} \cdot \mathbf{T}+i g / \frac{1}{2} B_{\mu} Y_{L}\right]\left(\begin{array}{c}
\nu_{e} \\
e
\end{array}\right)_{L}+ \\
& +i \bar{e}_{R}\left[i g \prime \frac{1}{2} B_{\mu} Y_{R}\right] e_{R}+-\frac{1}{4} \mathbf{W}_{\mu \nu} \cdot \mathbf{W}^{\mu \nu}-\frac{1}{4} B_{\mu \nu} \cdot B^{\mu}(2.10)
\end{aligned}
$$

where the first two terms describe the propagation of the free electron and neutrino, the third and fourth term the interaction of the left- and right-handed fermion fields with the gauge fields B and $\mathbf{W}$. The last two terms describe the propagation of the gauge fields. 
This model developed by Glashow, Weinberg and Salam in the 1960s [19] [20] [21] allows to describe electromagnetic and weak interactions in one framework. One therefore refers to it as unified electroweak theory.

The quark Lagrangian is similar to the lepton Lagrangian $\mathcal{L}_{l}$ eptons, the mass eigenstates of $\mathrm{d}, \mathrm{s}$ and $\mathrm{b}$ quarks being different from their eigenstates in weak interactions $d \prime, s /$ and bı. The transformation is done by the Cabibbo-Kobayashi-Maskawa (CKM) matrix.

$B_{\mu}$ cannot be identified with the $\gamma$, since the Lagrangian $\mathcal{L}_{\text {leptons }}$ would describe a coupling of the neutral neutrino with the photon. Therefore, the fields $\mathbf{W}_{\mu}$ and $B_{\mu}$ cannot be identified with the experimentally observed gauge bosons $\gamma, Z$ and $W^{ \pm}$. Instead, the following combinations have to be constructed to describe the gauge boson fields realized in nature:

$$
\begin{array}{r}
\mathbf{W}_{\mu}^{ \pm}=\frac{1}{\sqrt{2}}\left(W_{1 \mu} \pm i W_{2 \mu}\right) \\
\left(\begin{array}{c}
A_{\mu} \\
Z_{\mu}
\end{array}\right)=\left(\begin{array}{cc}
\cos \theta_{W} & \sin \theta_{W} \\
-\sin \theta_{W} & \cos \theta_{W}
\end{array}\right)\left(\begin{array}{c}
B_{\mu} \\
W_{3 \mu}
\end{array}\right)
\end{array}
$$

$\theta_{W}$ is the weak mixing angle, which is determined by the electromagnetic and weak coupling constants $g^{\prime}$ and $g$ :

$$
\cos \theta_{W}=\frac{g}{\sqrt{g^{2}+g^{\prime 2}}}
$$

The coupling constants are related to the electric charge by

$$
e=g \sin \theta_{W}=g^{\prime} \cos \theta_{W}
$$

The Lagrangian $\mathcal{L}_{l}$ eptons does not contain any mass terms for the fermion or gauge boson fields. In the SM, mass terms are introduced through spontaneous symmetry breaking.

\subsubsection{The Higgs Mechanism}

Mass terms such as $\frac{1}{2} M^{2} B_{\mu} B^{\mu}$ or $-m \bar{\Psi} \Psi$ are not gauge invariant and can not be added. To include massive particles into the model in a gauge invariant way the Higgs mechanism is used.

Four scalar fields $\Phi=\left(\phi^{+}, \phi^{0}\right)=\frac{1}{\sqrt{2}}\left(\phi_{1}^{+}+i \phi_{2}^{+}, \phi_{1}^{0}+i \phi_{2}^{0}\right)$ are added to the theory, and the term $\mathcal{L}_{H}=\left|D_{\mu} \Phi\right|^{2}-V\left(\Phi^{\dagger} \Phi\right)$ is added to $\mathcal{L}_{1}$. The Higgs potential has the form $V\left(\Phi^{\dagger} \Phi\right)=-\mu^{2}|\Phi|^{2}+\lambda|\Phi|^{4}$. By choosing $\mu^{2}<0$, the potential $V(\Phi)$ has a continuous minimum at $\pm \sqrt{\mu^{2} / 2 \lambda}$

$$
|\Phi|=\sqrt{\left(\phi^{+}\right)^{2}+\left(\phi^{0}\right)^{2}}=\sqrt{\mu^{2} / 2 \lambda}=v
$$

The ground state no longer shares the symmetry of the Lagrangian, hence the term spontaneous symmetry breaking. The vacuum expectation value of the field $\Phi$ is chosen to be

$$
\left(\begin{array}{l}
\phi^{+} \\
\phi^{0}
\end{array}\right)=\left(\begin{array}{l}
0 \\
v
\end{array}\right)
$$

to ensure the conservation of electromagnetic charge and a massless photon. 


\subsubsection{Quantum Chromodynamics}

Quantum chromodynamics (QCD) is the gauge field theory that describes the strong interactions of quarks and gluons. Its gauge group is $S U(3)_{C}$, the index $\mathrm{C}$ denoting the fact that quarks and gluons carry an additional charge called color. At tree level, the QCD Lagrangian consists of the following terms:

$$
\mathcal{L}_{Q C D}=\mathcal{L}_{\text {quark }}+\mathcal{L}_{\text {glue }}+\mathcal{L}_{\text {int }}
$$

The piece $\mathcal{L}_{\text {quark }}$ describes the propagation of free quarks,

$$
\mathcal{L}_{\text {quark }}=\sum_{q=u, d, s, \ldots} \frac{1}{2} \bar{\psi}_{q j}(x) \gamma^{\mu} \psi_{q}^{j}(x)
$$

$\mathcal{L}_{\text {glue }}$ describes the dynamics of the gluon fields and $\mathcal{L}_{\text {int }}$ the interaction between quarks and gluons. As quarks are spin $1 / 2$ particles, $\mathcal{L}_{\text {quark }}$ is the Dirac equation for each quark with given flavor and color, and is not invariant under local $S U(3)_{C}$ gauge transformations. To compensate for this, 8 'compensating' spin 1 fields are introduced which account for the gluons interacting with the quarks,

$$
\mathcal{L}_{\text {int }}=g_{s} \sum_{q=u, d, s, \ldots} \frac{1}{2} \bar{\psi}_{q j}(x)\left(\lambda^{a}\right)_{k}^{j} \gamma^{\mu} \psi_{q}^{k}(x) A_{\mu}^{a}(x),
$$

$G_{\mu}^{a}$ with $\mathrm{a}=1 \ldots 8$ denotes the gluon fields, $\lambda^{a}$ the generators of $\mathrm{SU}(3)$ and $g_{s}$ the coupling constant. The indices $\mathrm{k}$ and $\mathrm{j}$ denote the quark color and $\mathrm{q}$ the quark flavor. Like the group $\mathrm{SU}(2)$ of the electroweak interaction, $\mathrm{SU}(3)$ is a non-abelian group. The term $\mathcal{L}_{\text {glue }}$ describing the propagation of the color field thus is similar to the kinetic term of the fields $\mathbf{G}_{\mu}$,

$$
\mathcal{L}_{\text {glue }}=-\frac{1}{4} G_{\mu \nu}^{a} G^{a \mu \nu}
$$

with

$$
G_{\mu \nu}^{a}=\partial_{\mu} G_{\nu}^{a}-\partial_{\nu} G_{\mu}^{a}+g_{s} f^{a b c} G_{\mu}^{b} A^{c} \nu
$$

$f^{a b c}$ are the structure constants of $\mathrm{SU}(3)$ and $G_{\mu \nu}^{a}$ the gluon field-strength tensor. The gluon fields themselves carry color charge and interact with themselves, coupling gluons to gluons. The interaction of quarks and gluons change the color of a quark, thus the gluon color can be identified with a superposition of quark and anti quark colors. The QCD Lagrangian at tree level is given by

$$
\mathcal{L}_{Q C D}=\sum_{q=u, d, s, \ldots} \bar{\psi}_{q}\left(i D_{\mu} \gamma^{\mu}-m_{q}\right) \psi_{q}-\frac{1}{4} G_{\mu \nu}^{a} G^{a \mu \nu}
$$

with the covariant derivative $D_{\mu}=\partial_{\mu}-i g_{s} \frac{\lambda^{a}}{2} G_{\mu}^{a}$.

\subsection{Top Quark Production in Hadron Collisions}

In this section we discuss the production of top quarks in hadron collisions, limiting ourselves to Standard Model processes. 


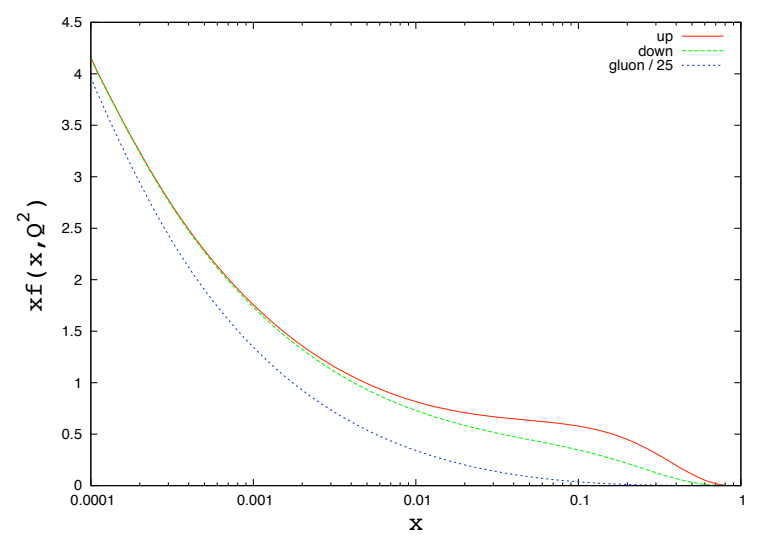

(a) Up and down quark and gluon PDF

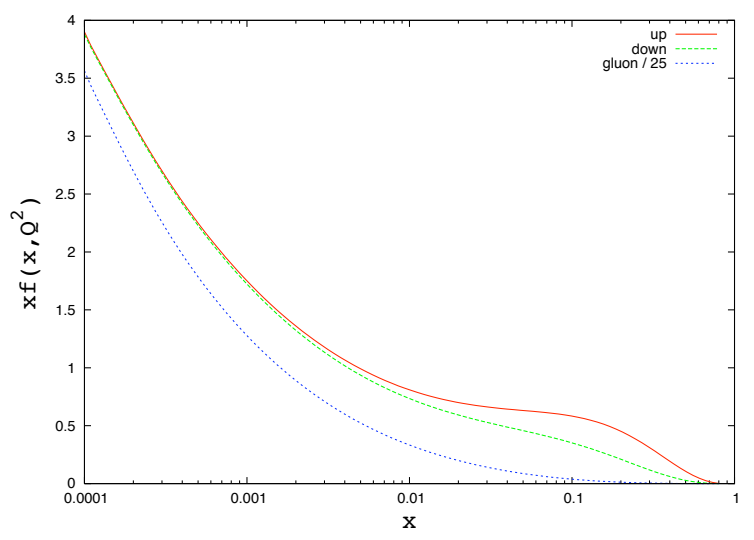

(b) Up and down quark and gluon PDF

Figure 2.1.: Parton densities for the up and down quark and the gluon in the proton according to CTEQ version 6.5 on the left hand side and MRST 2006 NNLO on the right hand side. $Q^{2}$ was set to $m_{\text {top }}^{2}=30625 \mathrm{GeV}^{2}$.

The two basic production processes at hadron colliders are the production of $t \bar{t}$ pairs which is dominated by the strong interaction, and the production of single top quarks due to electroweak interactions. The underlying theoretical framework is the parton model in which a high-energy hadron is regarded as a composition of quasi-free quarks and gluons that share the longitudinal hadron momentum. The cross section calculation is based on the factorization theorem stating that the cross section of a specific process is given by the convolution of the parton distribution functions (PDF) for the colliding hadrons and the hard parton-parton cross section for the process under consideration.

\subsubsection{Parton Distribution Functions}

The parton distribution functions are mostly determined from measurements of deep inelastic scattering experiments where either electrons, positrons or neutrinos collide with nucleons. However, input from fixed target experiments is still important. The PDFs $f_{i}\left(x_{i}, Q^{2}\right)$ are parametrized by the longitudinal momentum fraction $x_{i}=p_{i} / p_{A}$ of parton i in hadron $\mathrm{A}$ and the momentum transfer $Q^{2}$ in the interaction. The quantity $f_{i}\left(x_{i}, Q^{2}\right) d x$ is the probability that the parton $i$ carries a momentum fraction between $x$ and $x+d x$ of the hadron momentum, depending on $Q^{2}$.

The measured parton distribution functions span a wide region in $x$ and $Q^{2}$. In the high $x$ region, the valence quark densities are probed, whereas at low $x$ the PDFs are mostly sensitive to the gluon and sea quark densities. Several parametrizations of proton PDFs have been extracted from the experimental data by different groups of physicists, the most prominent being the ones derived by the CTEQ [22] [23] [24] [25] [26] [27] and MRST [28] 29] 30] 31] [32] 33] groups. Fig 2.1 compares density functions for the up and down quark and the gluon in the proton using two recent PDF sets, CTEQ 6.5 and MRST 2006. 


\subsubsection{The Factorization Ansatz}

To calculate the cross-section of the process $p \bar{p} \rightarrow t \bar{t}$ for the colliding protons $p$ and antiprotons $\bar{p}$, one convolutes the PDFs $f_{i, p}$ and $f_{j, \bar{p}}$ with the hard parton-parton cross section $\hat{\sigma}_{i j}, 34$

$$
\sigma(p \bar{p} \rightarrow t \bar{t})=\sum_{i, j=q, \bar{q}, g} \int f_{i, p}\left(x_{i}, \mu_{f}^{2}\right) f_{j, \bar{p}}\left(x_{j}, \mu_{f}^{2}\right) \cdot \hat{\sigma}_{i j}\left(i j \rightarrow t \bar{t} ; \hat{s} ; \alpha s\left(\mu_{r}^{2}\right)\right)
$$

The variable $\hat{s}$ denotes the square of the center-of-mass energy of the colliding partons, $\hat{s}=\left(p_{i}+p_{j}\right)^{2}=\left(x_{i} p_{A}+x_{j} p_{B}\right)^{2}$. At the Tevatron, where $p_{p}=p_{\bar{p}}=p$, we have $\hat{s}=$ $4 x_{i} x_{j} p^{2}=x_{i} x_{j} s$ with the Run II center-of-mass energy $\sqrt{s}=1.96 \mathrm{TeV}$ and $m_{p} \approx 0$. In analogy to QED, the fine structure constant $\alpha_{s}$ of the strong interaction is defined as [1]

$$
\alpha_{s}=\frac{g_{s}^{2}}{4 \pi}
$$

It is dependent on the energy scale of the partonic process, the so-called renormalization scale $\mu_{r}$. This scale has to be introduced to regulate divergent terms in the perturbation series used to calculate $\hat{\sigma}_{i j}$. This procedure introduces a dependence of the strength of the strong coupling on $\mu_{r},[12]$

$$
\alpha_{s}\left(\mu_{r}\right)=\frac{\alpha_{s}\left(\mu_{0}\right)}{1+\frac{\alpha_{s}\left(\mu_{0}\right)}{4 \pi}\left(11-\frac{2}{3} n_{f}\right)\left(\log \left(\mu_{r}^{2} / \mu_{0}^{2}\right)\right)}
$$

where $n_{f}$ is the number of quark flavors with a mass less than the energy scale $\mu_{0}$ at which $\alpha_{s}$ was determined. Finally, the factorization scale $\mu_{f}$ is associated with the separation of the hard scatter cross-section into a partonic part and the contributions from the proton and antiproton constituents. To some extend, $\mu_{r}$ and $\mu_{f}$ are arbitrary parameters, and most authors use only one scale $\mu=\mu_{f}=\mu_{r}$ in their calculations. For calculating heavy quark production, this scale is usually set to be on the order of the mass of the heavy quark, here $\mu=M_{\text {top }}$.

\subsubsection{The Parton-Parton Cross Section for Top Quark Pair Production}

The cross section of the process $i j \rightarrow t \bar{t}$, with partons $\mathrm{i}$ and $\mathrm{j}$ of the proton and antiproton, can be calculated with perturbative QCD. The leading order processes are quarkantiquark annihilation, t-channel top exchange and gluon-gluon-fusion. The leading order (Born) cross sections for heavy quark production have been calculated in the late 1970s [35] [36] [37]. The cross section for the process $q \bar{q} \rightarrow t \bar{t}$ is given by [38]

$$
\hat{\sigma}^{q \bar{q} \rightarrow t \bar{t}}(\hat{s})=\frac{8 \pi \alpha_{s}^{2}}{27 \hat{s}} \beta\left[1+\frac{\rho}{2}\right] \simeq \frac{4 \pi \alpha_{s}^{2}}{9 \hat{s}} \beta
$$

and similar for top pair production through gluon fusion,

$$
\hat{\sigma}^{g g \rightarrow t}\left(\hat{t}(\hat{s})=\frac{4 \pi \alpha_{s}^{2}}{12 \hat{s}}\left[\left(1+\rho+\frac{\rho^{2}}{16}\right) \ln \frac{1+\beta}{1-\beta}-\beta\left(\frac{7}{4}+\frac{31}{16} \rho\right)\right] \simeq \frac{7}{48} \frac{\pi \alpha_{s}^{2}}{\hat{s}} \beta\right.
$$


where $\rho=4 m_{t}^{2} / \hat{s} \leq 1$ and $\beta=\sqrt{1-\rho}$ is the velocity of the top quarks in the $t \bar{t}$ centerof-mass frame. Figs. 2.2 and 2.3 depict the leading order Feynman diagrams. The quarkantiquark annihilation diagram and the leftmost gluon fusion diagram correspond to schannel top quark pair production, where the momentum transfer from the initial to the final state is given by the Mandelstam-Variable $s, s=\left(p_{q}+p_{\bar{q}}\right)^{2}=\left(p_{t}+p_{\bar{t}}\right)^{2}$ or $s=$ $\left(p_{g_{1}}+p_{g_{2}}\right)^{2}=\left(p_{t}+p_{\bar{t}}\right)^{2}$ in the case of s-channel gluon fusion. The middle and rightmost gluon fusion diagram are the t-channel and u-channel diagram, respectively. In the tchannel the momentum transfer is given by $t=\left(p_{g_{2}}-p_{t}\right)^{2}=\left(p_{g_{1}}-p_{\bar{t}}\right)^{2}$, and finally the momentum transfer in the u-channel reads $u=\left(p_{g_{1}}-p_{t}\right)^{2}=\left(p_{g_{2}}-p_{\bar{t}}\right)^{2}$.

The threshold for top quark pair production occurs when $\beta \rightarrow 0$, or equally, $1-\rho \rightarrow 0$. The Tevatron operates near but not at the threshold for top pair production. Assuming both partons carry equal momentum $x_{i} \approx x_{j} \approx x_{\text {threshold }}$ where $x_{\text {threshold }} \approx 2 m_{t} / \sqrt{s}$, the center-of-mass energy of $\sqrt{s}=1.96 \mathrm{TeV}$ would lead to $x_{\text {threshold }} \approx 0.18$. For this fractional momentum, the quark parton distribution functions are significantly higher than the gluon distribution function. Thus, the process $q \bar{q} \rightarrow t \bar{t}$ is the dominant source of top quark pair production at the Tevatron, and $\hat{\sigma}^{q \bar{q} \rightarrow t \bar{t}}>\hat{\sigma}^{g g \rightarrow t \bar{t}}$.

Full calculations of the next-to-leading order (NLO) corrections to the inclusive partonparton cross section for $t \bar{t}$ production have been carried out [38]. Unlike the LO crosssection, the higher order corrections do not vanish near threshold. These and higher order corrections are predominantly due to soft gluon radiation that does not suffer the phase space suppression hard gluons do at threshold.

Theoretical calculations using standard perturbative techniques can predict the $t \bar{t}$ production cross-section up to NLO. However, higher order logarithmic corrections due to soft gluon emissions can be included through a technique called resummation [39] [40] [41] [42] [43]. These corrections can be expressed in a kinematic variable that represents the distance to the threshold, $x^{\text {th }}$, the order $\mathrm{n}$ of the QCD coupling constant and the exponent of the logarithm, l. The last two parameters describe the expansion of the resummed cross section in powers of $\alpha_{s}$. At threshold $x^{\text {th }} \rightarrow 0$, and the soft gluon corrections [44]

$$
\mathcal{D}_{l}\left(x^{t h}\right)=\left[\frac{\ln ^{l} x^{t h}}{x^{t h}}\right]_{+}
$$

become singular. At NLO, $\mathrm{n}=1$, we have leading $\mathcal{D}_{1}$ and next-to-leading $\mathcal{D}_{0}$ logarithms. At NNLO, there are leading $\mathcal{D}_{3}$, next-to-leading $\mathcal{D}_{2}$, next-to-next-to-leading $\mathcal{D}_{1}$ and nextto-next-to-next-to-leading $\mathcal{D}_{0}$ logarithms (NNNLL).

In principle, the soft logarithms can be resummed to all orders of $\alpha_{s}$, and the dependence of the result on the kinematics chosen would vanish at next-to-next-to-leading logarithmic level. Thus, the resummed NLO+NLL cross-section in [40] and the resummed NLO+NNLL cross-section in [43] do not depend on the kinematics chosen to estimate the distance from the $t \bar{t}$ production threshold.

However, expanding the resummed cross-section $\sigma^{\text {res }}$ to a fixed order in $\alpha_{s}$ while including subleading logarithms, leads to a reduced dependency on the renormalization scale $\mu_{r}$. However, the choice of kinematics is introduced as an additional uncertainty. In [42], $\sigma^{\text {res }}$ was expanded to $\mathcal{O}\left(\alpha_{s}^{4}\right)$ and including NNNLL.

Two kinematics schemes are examined, single particle inclusive (1PI) and pair-invariant 
mass (PIM) [42],

$$
\begin{aligned}
1 P I: i\left(p_{a}\right)+j\left(p_{b}\right) & \rightarrow t\left(p_{1}\right)+X\left(p_{2}\right) \\
P I M: i\left(p_{a}\right)+j\left(p_{b}\right) & \rightarrow t \bar{t}(M)+X(k)
\end{aligned}
$$

Here $i j=q \bar{q}$ or $i j=g g$. For the choice of 1 PI kinematics, $x^{t h}=s_{4}$, where

$$
s_{4}=s+t_{1}+u_{1}
$$

and

$$
\begin{aligned}
s & =\left(p_{a}+p_{b}\right)^{2} \\
t_{1} & =\left(p_{b}-p_{1}\right)^{2}-m_{t}^{2} \\
u_{1} & =\left(p_{a}-p_{1}\right)^{2}-m_{t}^{2}
\end{aligned}
$$

Thus,

$$
\begin{aligned}
s_{4} & =\left(p_{a}+p_{b}\right)^{2}+\left(p_{b}-p_{1}\right)^{2}-m_{t}^{2}+\left(p_{a}-p_{1}\right)^{2}-m_{t}^{2} \\
& =2 p_{a} p_{b}-2 p_{b} p_{1}-2 p_{a} p_{1}
\end{aligned}
$$

where we assumed the incoming quarks to be massless. The terms $p_{a} p_{1}$ and $p_{b} p_{1}$ go to zero with vanishing top quark momentum $p_{1}$.

When choosing PIM kinematics, $x^{t h}=1-M^{2} / s$, where $\mathrm{M}$ is the invariant mass of the top-antitop quark pair.

Recent NLO and soft gluon correction enhanced calculations using PDFs extracted by the CTEQ and MRST collaborations are available for top masses between 170 and 180 $\mathrm{GeV}$, with recent calculations using $165<m_{\text {top }}<180 \mathrm{GeV}$ [42] [45] 444] [43] [46].

The calculated top quark pair production cross section is given with two errors. The first error is determined by varying the factorization and renormalization scale to estimate the effect of uncalculated higher orders. It is common practice to estimate this scale dependences by taking $\mu_{f}=\mu_{r}=m_{t o p} / 2$ and $\mu_{f}=\mu_{r}=2 m_{t o p}$. The second error is due to the uncertainties of the data used to carry out the PDF fits. These errors are treated in a systematic way by a family of $n_{P D F}$ pairs of PDFs, one pair for each parameter used in the fit. Then, the uncertainty for the observable $O$ under consideration is estimated by

$$
\Delta O=\frac{1}{2} \sqrt{\sum_{k}\left(O_{k+}-O_{k-}\right)^{2}}
$$

where $O_{k \pm}$ are obtained using the parton distribution functions obtained by a statistical $\pm 1 \sigma$-variation of the $k$ th fit parameter, after diagonalizing the correlation matrix. To give an estimate of the total uncertainty, the uncertainty coming from the scale variation has to be combined with the uncertainty due to the PDF set chosen. Given their different origins - in one case the uncertainty due to missing higher orders, in the other case traces of the experimental uncertainty - the errors are combined linearly instead of adding them in quadrature to give a conservative estimate.

The most recent NLO-NLL and the approximate NNLO theory calculations are depicted in Fig. 2.4. 


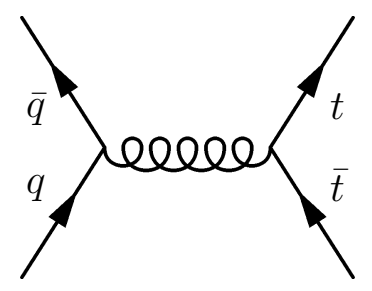

Figure 2.2.: Top quark pair production through quark-antiquark annihilation
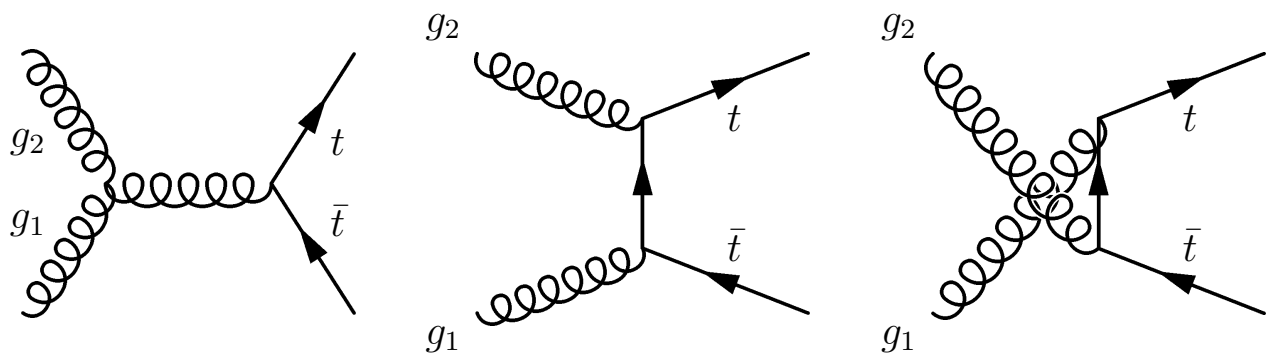

Figure 2.3.: Top quark pair production through gluon fusion 


\begin{tabular}{|c|c|c|c|c|}
\hline$m_{\text {top }} / \mathrm{GeV}$ & $r_{\mu}$ & $\sigma / p b$ & PDF used & $\begin{array}{l}\text { Order of expansion in } \alpha_{s} \\
\text { and logarithms taken into account }\end{array}$ \\
\hline & & & & NLL $^{\text {res }}$ calculations \\
\hline 170 & 0.5 & 7.97 & CTEQ6M & $\mathrm{NLO}+\mathrm{NLL}^{\text {res }}$ [45] \\
\hline 170 & 1.0 & 7.83 & CTEQ6M & NLO+NLL ${ }^{\text {res }} 45$ \\
\hline 170 & 2.0 & 7.29 & CTEQ6M & $\mathrm{NLO}+\mathrm{NLL}^{\text {res }}$ [45] \\
\hline 170 & 0.5 & 8.05 & MRST2002 & $\mathrm{NLO}+\mathrm{NLL}^{\text {res }} 45$ \\
\hline 170 & 1.0 & 7.91 & MRST2002 & $\mathrm{NLO}+\mathrm{NLL}^{\text {res }}$ 45] \\
\hline \multirow[t]{2}{*}{170} & 2.0 & 7.35 & MRST2002 & $\mathrm{NLO}+\mathrm{NLL}^{\text {res }}$ [45] \\
\hline & & & & Expansions of $\sigma^{\text {res }}$ beyond NLL to fixed order in $\alpha_{s}$ \\
\hline 175 & 0.5 & 7.01 & CTEQ6M & NNLO-NNNLL 1PI [42] \\
\hline 175 & 1.0 & 7.21 & CTEQ6M & NNLO-NNNLL 1PI [42] \\
\hline 175 & 2.0 & 7.04 & CTEQ6M & NNLO-NNNLL 1PI [42] \\
\hline 175 & 0.5 & 7.00 & MRST2002 & NNLO-NNNLL 1PI [42] \\
\hline 175 & 1.0 & 7.17 & MRST2002 & NNLO-NNNLL 1PI 42 ] \\
\hline 175 & 2.0 & 6.99 & MRST2002 & NNLO-NNNLL 1PI [42] \\
\hline 175 & 0.5 & 6.08 & CTEQ6M & NNLO-NNNLL PIM 42 \\
\hline 175 & 1.0 & 6.33 & CTEQ6M & NNLO-NNNLL PIM [42] \\
\hline 175 & 2.0 & 6.29 & CTEQ6M & NNLO-NNNLL PIM [42] \\
\hline 175 & 0.5 & 6.14 & MRST2002 & NNLO-NNNLL PIM [42] \\
\hline 175 & 1.0 & 6.35 & MRST2002 & NNLO-NNNLL PIM [42] \\
\hline \multirow[t]{2}{*}{175} & 2.0 & 6.28 & MRST2002 & NNLO-NNNLL PIM [42] \\
\hline & & & & Updated NLL Ues $^{\text {and NNLO approx. calculations }}$ \\
\hline 171 & 0.5 & 6.87 & CTEQ6.5 & NLO+NLL ${ }^{\text {res }}$ (Result in [40] as updated in [43]) \\
\hline 171 & 1.0 & 7.53 & CTEQ6.5 & $\mathrm{NLO}+\mathrm{NLL}^{\text {res }}$ (Result in [40] as updated in [43]) \\
\hline 171 & 2.0 & 7.78 & CTEQ6.5 & $\mathrm{NLO}+\mathrm{NLL}^{\text {res }}$ (Result in [40] as updated in [43]) \\
\hline 171 & 0.5 & 7.04 & MSRT2006 & $\mathrm{NLO}+\mathrm{NLL}^{\text {res }}$ (Result in [40] as updated in [43]) \\
\hline 171 & 1.0 & 7.79 & MSRT2006 & $\mathrm{NLO}+\mathrm{NLL}^{\text {res }}$ (Result in [40] as updated in [43]) \\
\hline 171 & 2.0 & 8.14 & MSRT2006 & $\mathrm{NLO}+\mathrm{NLL}^{\text {res }}$ (Result in [40] as updated in [43]) \\
\hline 171 & 0.5 & 7.65 & CTEQ6.5 & NNLO approx. [43] \\
\hline 171 & 1.0 & 7.93 & CTEQ6.5 & NNLO approx. [43] \\
\hline 171 & 2.0 & 7.99 & CTEQ6.5 & NNLO approx. 43] \\
\hline 171 & 0.5 & 7.90 & MSRT2006 & NNLO approx. [43] \\
\hline 171 & 1.0 & 8.23 & MSRT2006 & NNLO approx. 43] \\
\hline 171 & 2.0 & 8.31 & MSRT2006 & NNLO approx. 43 \\
\hline
\end{tabular}

Table 2.4.: Comparison of different theory predictions found in the literature. $r_{\mu}$ denotes the variation of the renormalization and factorization scale, i.e. $\mu_{r}=\mu_{f}=r_{\mu} \times$ $m_{\text {top }}$. 


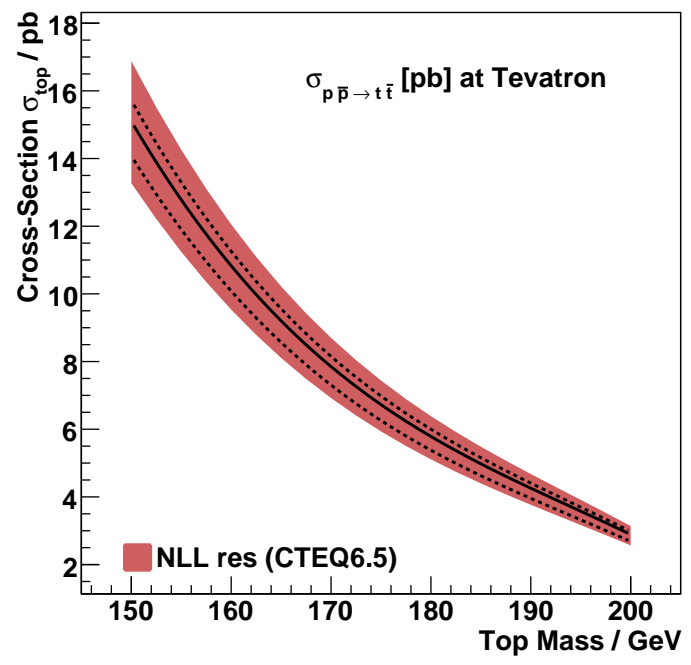

(a) Cacciari et. al. (CTEQ6.5)

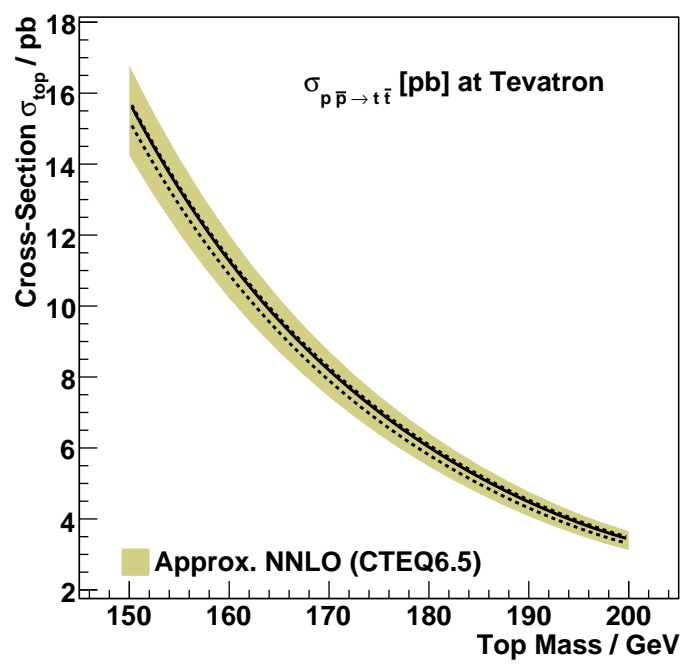

(c) Moch et. al. (CTEQ6.5)

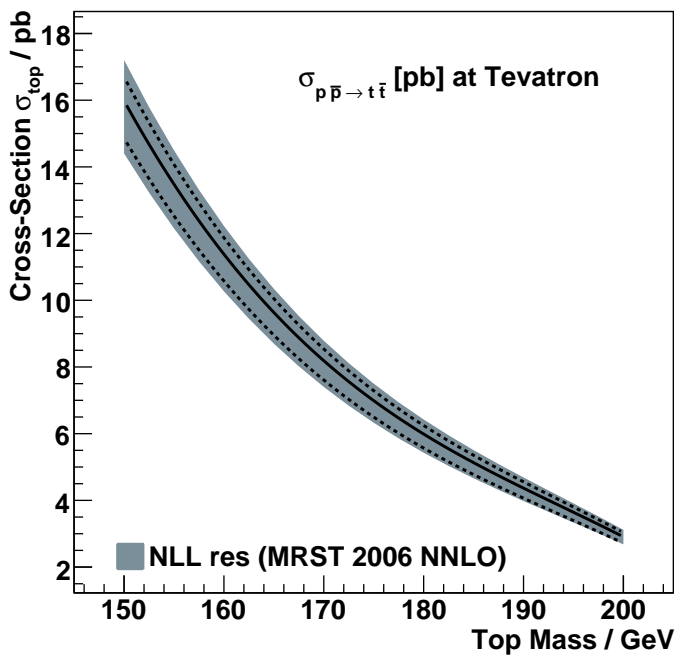

(b) Cacciari et. al. (MRST 2006 NNLO)

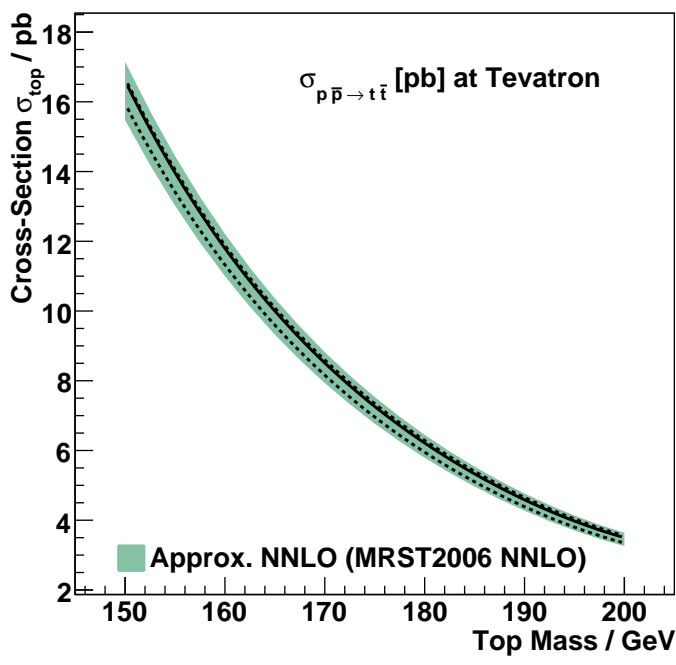

(d) Moch et. al. (MRST 2006 NNLO)

Figure 2.4.: Dependence of the NLO-NLL ${ }^{\text {res }}$ and approx. NNLO top quark pair production cross-section on the top mass. The cross-section was parametrized as a polynomial of order 5 . The parameters can be found in [47]. 


\subsection{Top Quark Decay}

In the SM top quarks decay dominantly into a $b$ quark and a $W$ boson, as depicted in Fig. 2.5. The decays to $d$ or $s$ quarks and a $W$ boson are suppressed relatively to $t \rightarrow b+W^{+}$ by factors of $\left|V_{t d}\right|^{2}$ and $\left|V_{t s}\right|^{2}$. The values of the CKM matrix elements $V_{t d}$ and $V_{t s}$ can be inferred from other measured matrix elements if we assume the CKM matrix to be unitary, $0.0048<V_{t d}<0.014$ and $0.037<V_{t s}<0.043$. From the available measurements of the CKM matrix elements, and again assuming the unitarity of the CKM matrix, we can infer that $V_{t b} \approx 1$. Thus we will only consider the decay $t \rightarrow b+W^{+}$in this and the following sections.

The different decay modes of the $W$ boson define the final states observed. There are three leptonic modes and six decay modes into quarks of different flavor. We distinguish four $t \bar{t}$ event signatures:

i. Both $W$ bosons decay into light leptons $(e, \mu)$ which can be directly seen in the detector. This channel is called dilepton channel.

ii. One $W$ decays into $e \nu_{e}$ or $\mu \nu_{\mu}$, the second decays into quarks. This category is called lepton-plus-jets.

iii. Both $W$ bosons decay into quarks. We refer to this as the all hadronic channel.

iv. At least one $W$ boson decays into a tau lepton, $\tau \nu_{\text {tau }}$ which itself decays leptonically. Depending on the decay of the 2 nd $W$ boson, the event is either classified as a dilepton event or lepton-plus-jets event.

v. At least one $W$ boson decays into a tau lepton, $\tau \nu_{\text {tau }}$ which itself decays hadronically into quarks. Depending on the decay of the 2nd $W$ boson, the event is either classified as a lepton+jets event or a hadronic event.

In good approximation we can neglect the lepton masses w.r.t. the $W$ mass, thus the decay width $\Gamma$ of the $W$ boson is equal for all leptons, $\Gamma_{W}^{0} \equiv \Gamma\left(W \rightarrow e \nu_{e}\right)=\Gamma\left(W \rightarrow \mu \nu_{\mu}\right)=$ $\Gamma\left(W \rightarrow \tau \nu_{\tau}\right)$. At lowest order in perturbation theory, the $W$ decay into $q \bar{q} \prime$ is given by the rate into leptons multiplied by the square of the CKM matrix element and enhanced by a color factor of $3, \Gamma(W \rightarrow q \bar{q} \prime)=3\left|V_{q q \prime}\right|^{2} \Gamma_{W}^{2}$. Since only $V_{u d}$ and $V_{c s}$ are of order one, the hadronic decay width of the $W$ is dominated by the decays $W \rightarrow u d$ and $W \rightarrow c s$ and one obtains $\Gamma_{\text {had }}=6 \Gamma_{W}^{0}$. Each leptonic channel has a branching fraction of $1 / 9$, while the hadronic channel into two quarks has a branching ratio of $6 / 9$. For the $t \bar{t}$ decay modes, we get thus the probabilities listed in table 2.5. At LO, the matrix element $\mathcal{M}$ of the decay $t \rightarrow b+W^{+}$is given by 34

$$
\mathcal{M}(t \rightarrow b+W)=\frac{i g}{\sqrt{2}} \bar{b} \notin^{W} \frac{1-\gamma_{5}}{5} t
$$

Neglecting terms of order $m_{b}^{2} / m_{t}^{2}, \alpha_{s}$ and $\left(\alpha_{s} / \pi\right) m_{W}^{2} / m_{t}^{2}$, the decay width in the SM is

$$
\Gamma_{t}=\frac{G_{F} m_{t}^{3}}{8 \pi \sqrt{2}}(1-y)^{2}(1+2 y) \times\left[1-\frac{2 \alpha_{s}}{3 \pi} f\right]
$$

with $y=\left(m_{W} / m_{t}\right)^{2}$ and $f=2 \pi^{2} / 3-5 / 2$. Taking into account first order QCD corrections, $f$ becomes dependent on $y, f(y)=2 \pi^{2} / 3-5 / 2-3 y+4.5 y^{2}-3 y^{2} \ln y$. The decay width increases from $1.07 \mathrm{GeV}$ at $m_{t}=160 \mathrm{GeV}$ to $1.53 \mathrm{GeV}$ at $m_{t}=180 \mathrm{GeV}$. 


\begin{tabular}{c||cccc} 
W decays & $e \nu_{e}$ & $\mu \nu_{\mu}$ & $\tau \nu_{\tau}$ & $q \bar{q}$ \\
\hline \hline$e \nu_{e}$ & $1.16^{-2}$ & $1.14^{-2}$ & $1.21^{-2}$ & $7.27^{-2}$ \\
$\mu \nu_{\mu}$ & $1.14^{-2}$ & $1.12^{-2}$ & $1.19^{-2}$ & $7.15^{-2}$ \\
$\tau \nu_{\tau}$ & $1.21^{-2}$ & $1.18^{-2}$ & $1.27^{-2}$ & $7.61^{-2}$ \\
$q \bar{q}$ & $7.27^{-2}$ & $7.14^{-2}$ & $7.61^{-2}$ & $4.57^{-1}$
\end{tabular}

Table 2.5.: Branching fractions of the $t \bar{t}$ event signature categories as calculated with the $W$ boson decay widths found in [18], $\Gamma\left(W \rightarrow e \nu_{e}\right)=0.1075, \Gamma\left(W \rightarrow \mu \nu_{\mu}\right)=$ $0.1057, \Gamma\left(W \rightarrow \tau \nu_{\tau}\right)=0.1125$ and $\Gamma(W \rightarrow q \bar{q})=0.676$.

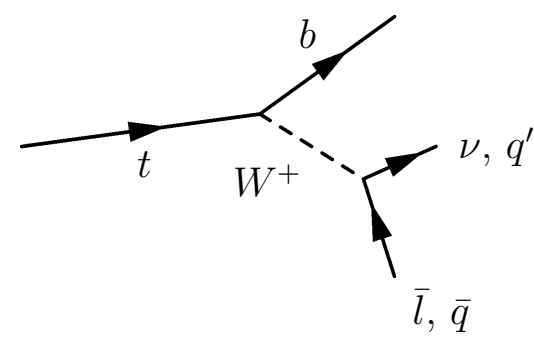

Figure 2.5.: Top quark decay to a $b$ quark and $W^{+}$, which in turn decays to two light fermions.

The large top decay rate implies a very short lifetime of $\tau_{t}=1 / \Gamma_{t} \approx 4 \cdot 10^{-25} s$ which is smaller than the characteristic formation time of hadrons, $\tau_{\text {form }} \approx 1 / \Lambda_{Q C D} \approx 2 \cdot 10^{-24}$. The small life time of the top quark neither allows for $t \bar{t}$ bound states nor for top-flavored hadrons to be formed. Therefore, we can assume top quarks being produced and decaying like free quarks. As a result, the top quark is the only quark where the polarization information is transferred to its decay products without being diluted by fragmentation effects.

\subsection{Top Quark Spin Correlation}

\subsubsection{Polarized Top Quark Production}

In unpolarized hadron collisions, the top and antitop quarks are produced with small angular correlations when summed over all events. As a result, measuring the opening angle of both leptons in dileptonic top quark pair decays, $\phi$, one gets an almost flat distribution,

$$
\frac{1}{\sigma} \frac{d \sigma}{d \cos \phi}=\frac{1-D \cos \phi}{2}
$$

with $\mathrm{D}=0.213$ at the Tevatron [48]. Though the net polarization is small, there is a large asymmetry in the rate for producing like-spin versus unlike-spin top quark pairs for the proper choice of spin quantization axis.

We define the correlation $C$ between the top and anti-top quark spin as

$$
C=4 \alpha_{i} \alpha_{i i}\left\langle\left(\hat{a} \cdot S_{t}\right)\left(\hat{b} \cdot S_{\bar{t}}\right)\right\rangle
$$


where $\hat{a}$ and $\hat{b}$ denote the spin quantization axis and $S_{t}$ and $S_{\bar{t}}$ the $t$ and $\bar{t}$ spin operators,

$$
S_{t}=\frac{1}{2}(\tau \otimes \mathbb{1})
$$

and

$$
S_{\bar{t}}=\frac{1}{2}(\mathbb{1} \otimes \tau)
$$

Here, $\tau$ is the spin operator acting on a single spin- $1 / 2$ particle, $\mathbb{1}$ is the unit operator and $\otimes$ is the tensor product. Thus, the operators $S_{t}$ and $S_{\bar{t}}$ act each on a spin-1/2 particle of a coupled two particle system. The factor $\alpha_{i}\left(\alpha_{i i}\right)$ is the spin analyzing power of the $i$ th top (antitop) decay product (see below). Choosing the helicity basis, where $\hat{a}$ and $\hat{b}$ are the top and antitop momentum vector, respectively, and defining a right-handed top quark to have its spin parallel to its velocity vector, one has for the double spin asymmetry

$$
4\left\langle\left(\hat{a} \cdot S_{t}\right)\left(\hat{b} \cdot S_{\bar{t}}\right)\right\rangle=\frac{\sigma\left(t_{R} \bar{t}_{R}\right)+\sigma\left(t_{L} \bar{t}_{L}\right)-\sigma\left(t_{R} \bar{t}_{L}\right)-\sigma\left(t_{L} \bar{t}_{R}\right)}{\sigma\left(t_{R} \bar{t}_{R}\right)+\sigma\left(t_{L} \bar{t}_{L}\right)+\sigma\left(t_{R} \bar{t}_{L}\right)+\sigma\left(t_{L} \bar{t}_{R}\right)}
$$

In general, denoting $\hat{a}$ parallel $s_{t}$ (and $\hat{b}$ parallel $s_{\bar{t}}$ ) with $\uparrow$ and $\hat{a}$ antiparallel $s_{t}$ (and $\hat{b}$ antiparallel $s_{\bar{t}}$ ) with $\downarrow$, the double spin asymmetry reads

$$
4\left\langle\left(\hat{a} \cdot S_{t}\right)\left(\hat{b} \cdot S_{\bar{t}}\right)\right\rangle=\frac{\sigma\left(t_{\uparrow} \bar{t}_{\uparrow}\right)+\sigma\left(t_{\downarrow} \bar{t}_{\downarrow}\right)-\sigma\left(t_{\uparrow} \bar{t}_{\downarrow}\right)-\sigma\left(t_{\downarrow} \bar{t}_{\uparrow}\right)}{\sigma\left(t_{\uparrow} \bar{t}_{\uparrow}\right)+\sigma\left(t_{\downarrow} \bar{t}_{\downarrow}\right)+\sigma\left(t_{\uparrow} \bar{t}_{\downarrow}\right)+\sigma\left(t_{\downarrow} \bar{t}_{\uparrow}\right)}
$$

In quark-antiquark annihilation the $t \bar{t}$ quark pair is produced via a $J=1$ s-channel gluon. Near the production threshold, the $t \bar{t}$ pair has zero angular momentum, so the $t \bar{t}$ pair is in a triplet $\left({ }^{3} S_{1}\right)$ state, with the spin eigenstates

$$
\begin{gathered}
\mid++> \\
\frac{1}{\sqrt{2}}[|+->+|-+>] \\
\mid-->
\end{gathered}
$$

as depicted on the left-hand side of Fig. 2.6. The ${ }^{1} S_{0}$ state with $J=0$ is forbidden due to angular momentum conservation. Thus $2 / 3$ of the top quark pairs produced have like spin and opposite helicity. The helicity of a particle is given by its spin projected onto its momentum three-vector. Far above threshold, helicity conservation at high energy ensures that the top and anti-top quarks are produced with opposite helicity, or same spin. The relation [49]

$$
\frac{\sigma\left(t_{R} \bar{t}_{L}+t_{L} \bar{t}_{R}\right)}{\sigma\left(t_{R} \bar{t}_{R}+t_{L} \bar{t}_{L}\right)}=2 \frac{M_{t \bar{t}}^{2}}{4 m_{t}^{2}}
$$

interpolates between the two regimes. Fig 2.7 illustrates the spin alignment at $t \bar{t}$ production threshold and far above. On the other hand, top quark pairs produced via gluon fusion near threshold are in a ${ }^{1} S_{0}$ state (as shown on the right-hand side of Fig. 2.6.)

$$
\frac{1}{\sqrt{2}}[|+->-|-+>]
$$

and are of unlike spin with a correlation of 100\%. In QCD, parity and CP are conserved, and therefore the relations $\sigma\left(t_{R} \bar{t}_{L}\right)=\sigma\left(t_{L} \bar{t}_{R}\right)$ and $\sigma\left(t_{R} \bar{t}_{R}\right)=\sigma\left(t_{L} \bar{t}_{L}\right)$ hold. 

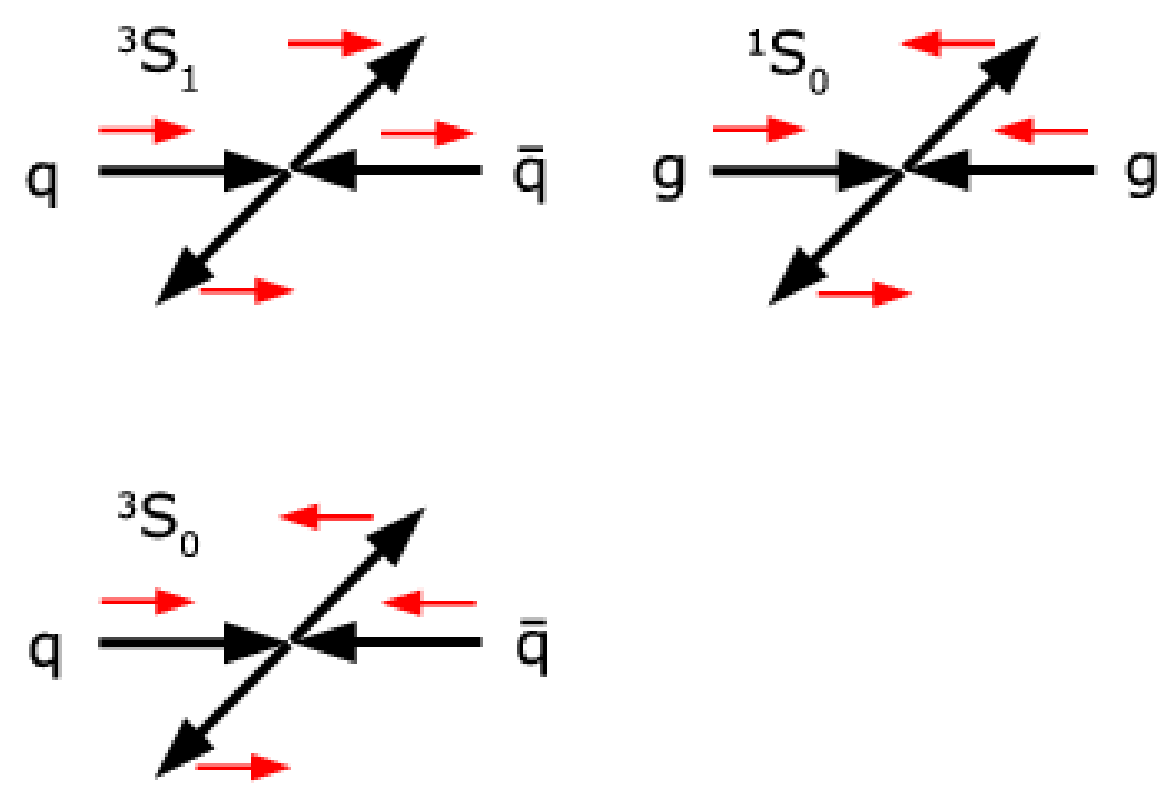

Figure 2.6.: Spin configuration of top and antitop quark pairs produced via quarkantiquark-annihilation (left) and gluon fusion (right). For the quark-antiquarkannihilation, the likespin (top) and unlikespin (bottom) states of the ${ }^{3} S$ state are shown.
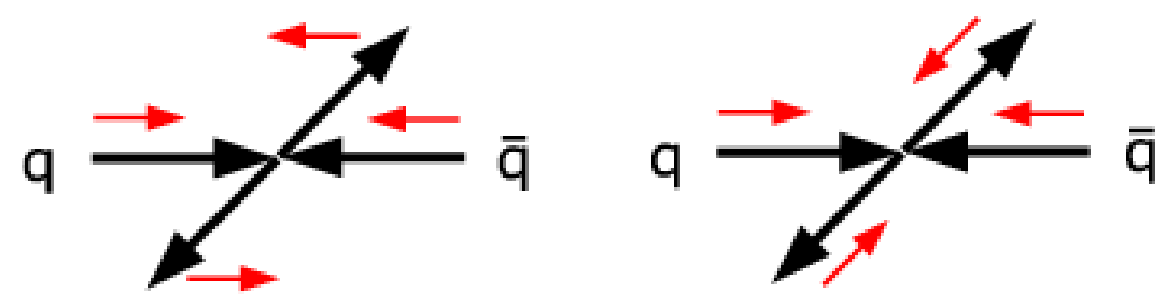

Figure 2.7.: Spin alignment of top and antitop quark pairs at the production threshold (left) and well above (right). 
Representing the particle momentum by its symbol and decomposing the top and antitop quark momentum into a sum of two massless momenta $\left(t=t_{1}+t_{2}\right.$ and $\left.\bar{t}=\bar{t}_{1}+\bar{t}_{2}\right)$, such that in the rest frame of the top (anti-top) the spatial momentum of $t_{1}\left(\bar{t}_{1}\right)$ defines the spin axis for the top (anti-top) quark, the squared helicity amplitudes for $q \bar{q} \rightarrow t \bar{t}$ and $g g \rightarrow t \bar{t} \operatorname{read}([50]$, [51])

$$
\begin{aligned}
& \sum_{\uparrow \uparrow, \downarrow \downarrow}|\mathcal{M}(q \bar{q} \rightarrow t \bar{t})|^{2}=\frac{16 g^{4}}{(2 q \cdot \bar{q})^{2}} \times \\
& {\left[\left(2 q \cdot t_{1}\right)\left(2 \bar{q} \cdot \bar{t}_{2}\right)+\left(2 q \cdot \bar{t}_{1}\right)\left(2 \bar{q} \cdot t_{2}\right)+\frac{1}{m_{t}^{2}} \operatorname{Tr}\left(q t_{1} t_{2} \bar{q} \bar{t}_{2} \bar{t}_{1}\right)\right]+(q \leftrightarrow \bar{q})} \\
& \sum_{\uparrow \downarrow, \downarrow \uparrow}|\mathcal{M}(q \bar{q} \rightarrow t \bar{t})|^{2}=\frac{16 g^{4}}{(2 q \cdot \bar{q})^{2}} \times \\
& {\left[\left(2 q \cdot t_{1}\right)\left(2 \bar{q} \cdot \bar{t}_{1}\right)+\left(2 q \cdot \bar{t}_{2}\right)\left(2 \bar{q} \cdot t_{2}\right)+\frac{1}{m_{t}^{2}} \operatorname{Tr}\left(q t_{1} t_{2} \bar{q}_{1} \bar{t}_{2}\right)\right]+(q \leftrightarrow \bar{q})} \\
& \sum_{\uparrow \uparrow, \downarrow \downarrow}|\mathcal{M}(g g \rightarrow t \bar{t})|^{2}= \\
& \frac{4}{3} g^{4}\left\{\frac{4}{\left(t \cdot g_{1}\right)^{2}}-\frac{1}{\left(t \cdot g_{1}\right)\left(t \cdot g_{2}\right)}+\frac{4}{\left(t \cdot g_{2}\right)^{2}}\right\}\left\{m_{t}^{2}\left[\left(2 t_{1} \cdot \bar{t}_{1}\right)+\left(2 t_{2} \bar{t}_{2}\right)\right]-\frac{\operatorname{Tr}\left(g_{1} t g_{2} \bar{t}\right)}{\left(2 g_{1} \cdot g_{2}\right)^{2}} \times\right. \\
& \left.\left[\left(2 g_{1} \cdot t_{1}\right)\left(2 g_{2} \cdot \bar{t}_{2}\right)+\left(2 g_{1} \cdot \bar{t}_{1}\right)\left(2 g_{2} \cdot t_{2}\right)+\frac{1}{m_{t}^{2}} \operatorname{Tr}\left(g_{1} t_{1} t_{2} g_{2} \bar{t}_{2} \bar{t}_{1}\right)\right]\right\}+\left(g_{1} \leftrightarrow g_{2}\right) \\
& \sum_{\uparrow \downarrow, \downarrow \uparrow}|\mathcal{M}(g g \rightarrow t \bar{t})|^{2}= \\
& \frac{4}{3} g^{4}\left\{\frac{4}{\left(t \cdot g_{1}\right)^{2}}-\frac{1}{\left(t \cdot g_{1}\right)\left(t \cdot g_{2}\right)}+\frac{4}{\left(t \cdot g_{2}\right)^{2}}\right\}\left\{m_{t}^{2}\left[\left(2 t_{1} \cdot \bar{t}_{2}\right)+\left(2 \bar{t}_{1} t_{2}\right)\right]-\frac{\operatorname{Tr}\left(g_{1} t g_{2} \bar{t}\right)}{\left(2 g_{1} \cdot g_{2}\right)^{2}} \times\right. \\
& \left.\left[\left(2 g_{1} \cdot t_{1}\right)\left(2 g_{2} \cdot \bar{t}_{1}\right)+\left(2 g_{1} \cdot \bar{t}_{2}\right)\left(2 g_{2} \cdot t_{2}\right)+\frac{1}{m_{t}^{2}} \operatorname{Tr}\left(g_{1} t_{1} t_{2} g_{2} \bar{t}_{2} \bar{t}_{2}\right)\right]\right\}+\left(g_{1} \leftrightarrow g_{2}\right)
\end{aligned}
$$

In the above equations, $\uparrow \uparrow$ denotes parallel top and antitop quark up spins, $\uparrow \downarrow$ the spin up top quark and spin down anti-quark configuration, $\downarrow \uparrow$ the spin down top quark and spin up anti-quark configuration and $\downarrow$ is an abbreviation for parallel top and antitop quark down spins.

Though Eqns. 2.50 - 2.52 hold for arbitrary decompositions of $t$ into $t_{1}$ and $t_{2}$ and $\bar{t}$ into $\bar{t}_{1}$ and $\bar{t}_{2}$, each of the helicity amplitudes is dependent on the choice of spin axis $t_{1}\left(\bar{t}_{1}\right)$. Therefore the ratio of like-spin to unlike-spin production rate is dependent on the decomposition of $t(\bar{t})$, and not all decompositions are equally effective for extracting the spin correlation. At the Tevatron, the optimal spin axis is the so-called "off-diagonal" axis that makes use of the zero momentum frame of the incoming partons [52]. However, the angle between the incoming partons and the produced top quarks is not accessible at hadron colliders, where the direction of the beamline $\hat{p}$ is a sensible choice for the spin axis, $\hat{a}=\hat{b}=\hat{p}$. Compared to the off-diagonal axis, with which a spin correlation $\mathrm{C}$ of 0.937 
is predicted by theory with the above LO matrix elements, choosing the beamline basis a spin correlation $\mathrm{C}$ of 0.928 is predicted. In this basis, the helicity amplitudes $2.50-2.52$ become ([50], [51])

$$
\begin{aligned}
& \sum_{\uparrow \uparrow, \downarrow \downarrow}|\mathcal{M}(q \bar{q} \rightarrow t \bar{t})|^{2}=8 g^{4} \frac{\beta^{2}\left(1-\beta^{2}\right) \sin ^{2} \theta^{*}}{\left(1-\beta \cos \theta^{*}\right)^{2}} \\
& \sum_{\uparrow \downarrow, \downarrow \uparrow}|\mathcal{M}(q \bar{q} \rightarrow t \bar{t})|^{2}=8 g^{4}\left[1+\frac{\left(1-\beta \cos \theta^{*}-\beta^{2} \sin ^{2} \theta^{*}\right)^{2}}{\left(1-\beta \cos \theta^{*}\right)^{2}}\right] \\
& \sum_{\uparrow \uparrow, \downarrow \downarrow}|\mathcal{M}(g g \rightarrow t \bar{t})|^{2}= \\
& \quad \frac{16}{3} g^{4} \mathcal{Y}\left(\beta, \cos \theta^{*}\right)\left(1-\beta^{2}\right)\left[1+\beta^{2} \cos ^{2} \theta^{*}+2 \beta^{3} \sin ^{2} \theta^{*} \frac{\beta-\cos \theta^{*}}{\left(1-\beta \cos \theta^{*}\right)^{2}}\right] \\
& \sum_{\uparrow \downarrow, \downarrow \uparrow}|\mathcal{M}(g g \rightarrow t \bar{t})|^{2}= \\
& \frac{16}{3} g^{4} \mathcal{Y}\left(\beta, \cos \theta^{*}\right) \beta^{2} \sin ^{2} \theta^{*}\left[1+\frac{\left(1-\beta^{2}\right)^{2}+\left(1-\beta \cos \theta^{*}-\beta^{2} \sin ^{2} \theta^{*}\right)^{2}}{\left(1-\beta \cos \theta^{*}\right)^{2}}\right]
\end{aligned}
$$

with

$$
\mathcal{Y}\left(\beta, \cos \theta^{*}\right)=\frac{7+9 \beta^{2} \cos ^{2} \theta^{*}}{\left(1-\beta^{2} \cos ^{2} \theta^{*}\right)^{2}}
$$

and the angle $\theta^{*}$ between the top quark and the left-moving beam. While the like-spin pair quark annihilation amplitude is suppressed for both small and large $\beta$ by $\beta^{2}\left(1-\beta^{2}\right)$, the unlike-spin quark annihilation amplitude contains a contribution that is independent of $\beta$. In the helicity basis, where the direction of flight of the top quark is the spin quantization axis, a spin correlation $\mathrm{C}$ of -0.471 is predicted by theory for measurements performed at the Tevatron. However, it is the best choice for a quantization axis at LHC energies. In this basis, the helicity amplitudes read ([50], [51])

$$
\begin{aligned}
\sum_{L L, R R}|\mathcal{M}(q \bar{q} \rightarrow t \bar{t})|^{2} & =8 g^{4}\left(1-\beta^{2}\right) \sin ^{2} \theta^{*} \\
\sum_{L R, R L}|\mathcal{M}(q \bar{q} \rightarrow t \bar{t})|^{2} & =8 g^{4}\left(1+\cos ^{2} \theta^{*}\right) \\
\sum_{L L, R R}|\mathcal{M}(g g \rightarrow t \bar{t})|^{2} & =\frac{16}{3} g^{4} \mathcal{Y}\left(\beta, \cos \theta^{*}\right)\left(1-\beta^{2}\right)\left(1+\beta^{2}+\beta^{2} \sin ^{4} \theta^{*}\right) \\
\sum_{L L, R R}|\mathcal{M}(g g \rightarrow t \bar{t})|^{2} & =\frac{16}{3} g^{4} \mathcal{Y}\left(\beta, \cos \theta^{*}\right) \beta^{2} \sin ^{2} \theta^{*}\left(1+\cos ^{2} \theta^{*}\right)
\end{aligned}
$$

with $\mathcal{Y}$ and $\theta^{*}$ as defined above.

\subsubsection{Constructing the Top Rest Frame}

To measure the direction of the top quark spin, the direction of the $i$ th decay product of the top (antitop) in the top (antitop) rest frame has to be compared with the chosen quantization axis. Therefore, we first describe hot to construct this rest frame before discussing the decay of polarized top quarks. 
A prescription how to construct the top and anti-top rest frames in a soft and collinear safe way has to be given. Moreover, the specific prescription has to be unique.

An obvious frame to start with is the center-of-mass frame of the incoming partons. However, this frame can only be constructed if the four-momenta of all final state particles and jets is known. In particular, at NLO QCD, one needs to know in addition to the top and anti-top momenta the momentum of the hard gluon emitted in real radiation. This information cannot be obtained if the gluon is collinear to one of the initial partons. Thus the center-of-mass frame is not collinear safe and cannot be applied beyond leading order. This holds also for other observables involving the four-momenta of the top and anti-top defined in the center-of-mass frame of the initial state partons.

A suitable choice is the zero momentum frame $(Z M F)$ of the $t \bar{t}$ quarks. In this frame,

$$
(t+\bar{t})^{\mu}=\left(\sqrt{(t+\bar{t})^{2}}, 0,0,0\right) .
$$

In principle, this frame is only defined up to a rotation. This can be resolved by choosing the axis of the incoming hadron beams as z-axis and one orthogonal direction as the $\mathrm{x}$-axis. After having measured the top and anti-top momenta in this laboratory frame, the $t \bar{t}$-ZMF can be defined unambiguously by a rotation-free Lorentz boost. Now the rest frames of the top and anti-top can be defined by a rotation-free Lorentz boost from the $t \bar{t}$-ZMF.

\subsubsection{Polarized Top Quark Decay}

Because of the extremely short top quark lifetime, the top quark decays before hadronization, passing on its spin information to its decay products. The squared matrix element of the decay chain is rather simple, considering the three-body final state. Again, we decompose the top quark momentum into two massless momenta $t_{1}$ and $t_{2}$ in a way the spatial momentum of $t_{1}$ defines the spin axis in the top rest frame. For a top quark $t$ decaying into a $b$ quark, a positron $\bar{e}$ and a neutrino $\nu$, the matrix elements for the decay of spin up and spin down top quarks read [51]

$$
\begin{aligned}
\left|\mathcal{M}_{\uparrow}\left(t \rightarrow b \bar{e} \nu_{e}\right)\right|^{2} & =\frac{g_{W}^{4}(2 \nu \cdot b)\left(2 \bar{e} \cdot t_{2}\right)}{\left(2 \nu \cdot \bar{e}-M_{W}^{2}\right)^{2}+M_{W}^{2} \Gamma_{W}^{2}}, \\
\left|\mathcal{M}_{\downarrow}\left(t \rightarrow b \bar{e} \nu_{e}\right)\right|^{2} & =\frac{g_{W}^{4}(2 \nu \cdot b)\left(2 \bar{e} \cdot t_{1}\right)}{\left(2 \nu \cdot \bar{e}-M_{W}^{2}\right)^{2}+M_{W}^{2} \Gamma_{W}^{2}}
\end{aligned}
$$

For the decay $t \rightarrow b \bar{d} u$, one replaces $\bar{e}$ with $\bar{d}$ and $\nu$ with $\mathrm{u}$ in the above expressions. With the above LO matrix elements, the differential decay rate may be computed, parametrized in terms of the angle between the chosen spin axis and the direction of flight of the $i$ th decay product in the top quark rest frame, $\theta_{i}$, as [51]

$$
\frac{1}{\Gamma} \frac{d \Gamma}{d\left(\cos \theta_{i}\right)}=\frac{1+\alpha_{i} \cos \theta_{i}}{2}
$$

where $\Gamma$ is the total top decay width and $\frac{d \Gamma}{d\left(\cos \theta_{i}\right)}$ the differential top decay width with respect to the angle between the $i$ th top decay product and the top spin quantization axis. The correlation coefficient $\alpha$ can be calculated from the matrix elements [51 (2.65) and (2.64). For a spin up quark, the results are given in Table 2.6 and depicted in Fig. 2.8. The spin down top quark has correlation coefficients of opposite sign to the spin up top 


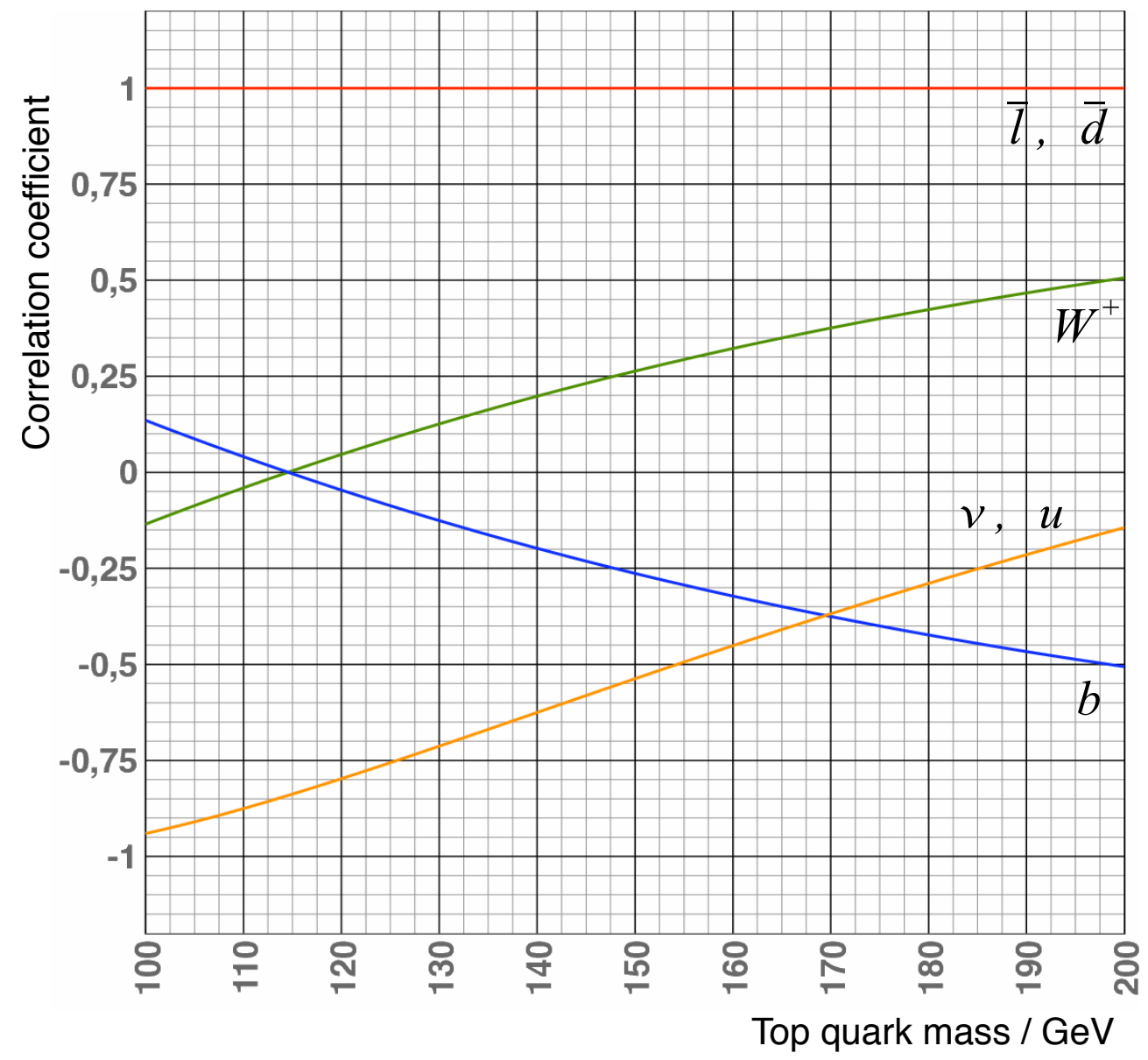

Figure 2.8.: Dependence of the spin analyzing power of the top quark decay products on the top quark mass. The analyzing power for the lepton and the down-type quark is constant for all top masses. 


\begin{tabular}{c|c|c} 
Top quark decay product & Correlation coefficient & Coefficient for $m_{\text {top }}=170 \mathrm{GeV} / \mathrm{c}^{2}$ \\
\hline \hline $\bar{\ell}$ or $\bar{d}, \bar{s}$ & 1 & 1 \\
$\nu$ or $\mathrm{u}, \mathrm{c}$ & $\frac{(\xi-1)\left(\xi^{2}-11 \xi-2\right)+12 \xi \ln \xi}{(\xi+2)(\xi-1)^{2}}$ & -0.3584 \\
$\mathrm{~W}^{+}$ & $\frac{\xi-2}{\xi+2}$ & 0.3816 \\
$\mathrm{~b}$ & $-\frac{\xi-1}{\xi+2}$ & -0.3816
\end{tabular}

Table 2.6.: Analyzing power $\alpha$ for decay products of both semileptonic and hadronic top quark decays as a function of $\xi=m_{t}^{2} / m_{W}^{2}$.

quark, and the anti-top quark spin up (down) correlation coefficients are the same as the top quark spin down (up) coefficients. The double differential cross-section for the decay of a $t \bar{t}$ quark pair is given by ([51], [48])

$$
\frac{1}{\sigma} \frac{d^{2} \sigma}{d\left(\cos \theta_{i}\right) d\left(\cos \theta_{\bar{i}}\right)}=\frac{1+C \cos \theta_{i} \cos \theta_{\bar{i}}}{4}
$$

where $\mathrm{C}$ is given by Eq. 7.3 , and $\theta_{i}\left(\theta_{\bar{i}}\right)$ is again the angle between the chosen spin quantization axis and the $i$ th decay product of the top (antitop) quark in the top (antitop) rest frame. 


\section{Experimental Apparatus}

Fermi National Accelerator Laboratory (Fermilab; FNAL) is the home of the Tevatron collider. Protons and Antiprotons are accelerated and collide at a center-of-mass energy of $\sqrt{s}=1.96 \mathrm{TeV}$. Two multi-purpose experiments, D $\varnothing$ and CDF, are located at the interaction points where the proton and antiproton beams intersect. At the end of the first data taking period (Run I) that lasted from 1992 to 1996 the top quark was discovered. Before the startup of the second data taking period, Run II, both the experiments and the accelerator were upgraded extensively. Up to May 2006 each experiment has collected data corresponding to an integrated Luminosity of $\mathcal{L} \approx 1.3 \mathrm{fb}^{-1}$. By the end of Run II, an integrated luminosity of $8 \mathrm{fb}^{-1}$ per experiment is expected. The analysis at hand is based on data collected from April 2002 to February 2006 (Run IIa) by the DØ detector. The following chapter describes the accelerator chain used for Run II and the DØ detector.

\subsection{The Fermilab Accelerator Complex}

The Fermilab accelerator complex consists of numerous stages of acceleration and storage of protons, and creation, acceleration and storage of antiprotons (cf. Fig. 3.1). More detailed information about the operations of the accelerator chain and the accelerators themselves can be found in [53] and [54]. In the first stage, hydrogen ions $H^{-}$are created and accelerated to $0.75 \mathrm{MeV}$ using a Cocroft-Walton accelerator. A linear accelerator increases the energy of the $H^{-}$ions to $400 \mathrm{MeV}$ before the electrons are stripped off. The Booster, a circular synchrotron, groups the protons into bunches and accelerates them to $8 \mathrm{GeV}$. In the next step of the accelerator chain, the proton bunches are transferred to the Main Injector, where they are accelerated to $120 \mathrm{GeV}$. Antiprotons are produced in a hadronic reaction by shooting proton bunches from the Main Injector to a copper/nickel target. Antiproton production is the limiting factor for increasing the luminosity at the Tevatron, since on average only 15 antiprotons are collected from every million protons that collide with the target. In the Debuncher, the bunch structure of the antiprotons, a remnant from the Main Injectors proton bunches, is removed and the antiprotons are stochastically cooled and their energies are made uniform. The Accumulator stores the antiprotons. The Main Injector tunnel also holds the Recycler, a storage ring that is directly above the Main Injector beamline. It plays a crucial role in increasing the antiproton rate by acting as a post-accumulator for recycled antiprotons from the previous Tevatron store.

When a sufficient number of antiprotons is available in the Accumulator and the Recycler (typically $150-200 \times 10^{10}$ antiprotons), 36 bunches of protons are transferred from the Main Injector to the Tevatron at $150 \mathrm{GeV}$. Antiprotons from the Accumulator are loaded into the Main Injector, 4 bunches a time, where they are accelerated to $150 \mathrm{GeV}$ and injected into the Tevatron. After injecting 36 antiproton bunches into the Tevatron, both the proton and antiproton beams are accelerated to the maximum beam energy of $980 \mathrm{GeV}$. In the Tevatron synchrotron, protons and antiprotons are separated into 3 super bunches $2.6 \mu \mathrm{s}$ 


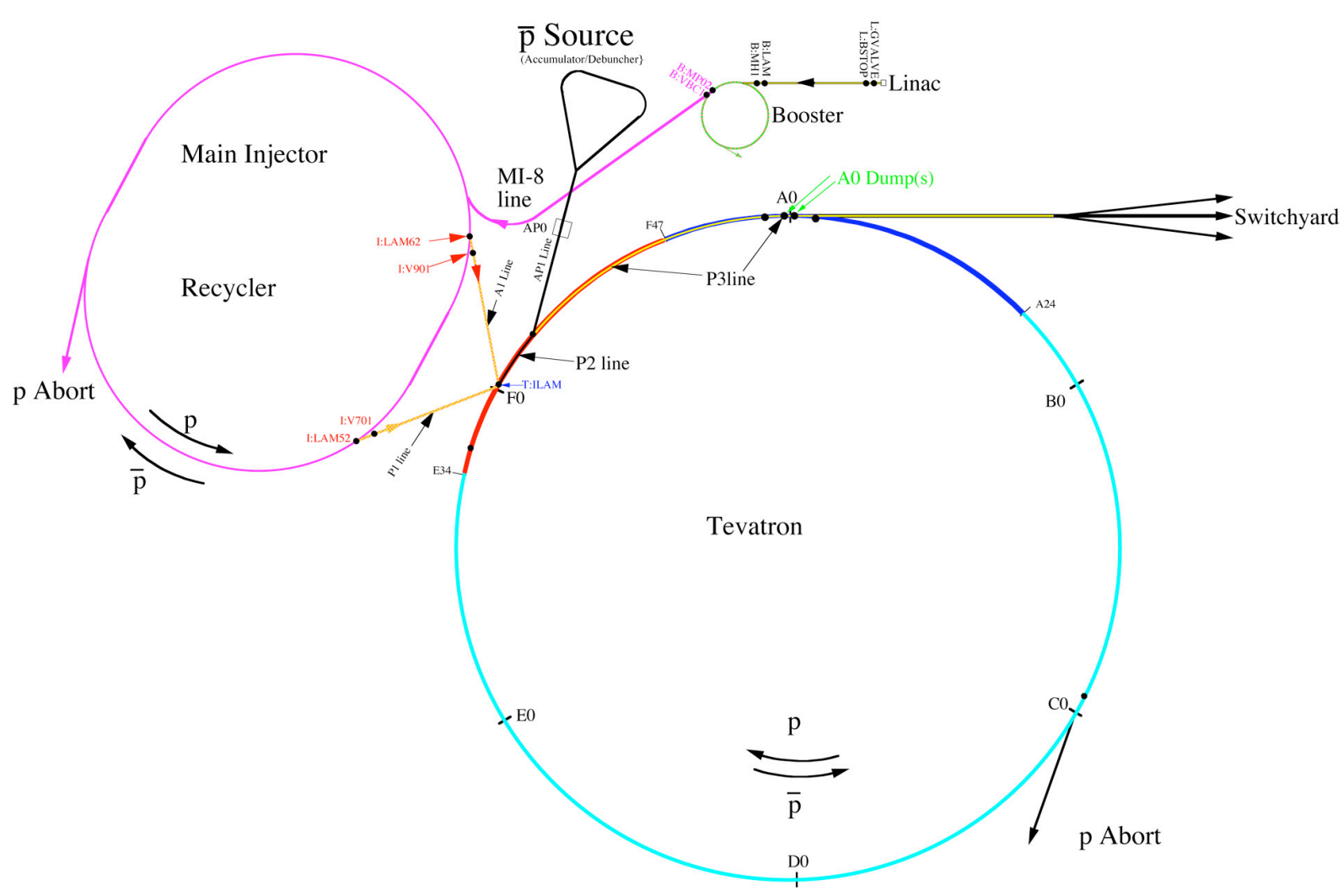

Figure 3.1.: Map of the accelerator complex at FNAL (from Ref. [53]).

apart. Each super bunch consists of 12 bunches separated by 396 ns, each bunch having a length of about $50 \mathrm{~cm}$ and a radius of about $2.5 \times 10^{-6} \mathrm{~cm}$. The beams are collided at two intersection points, each inside one multi-purpose detector, DØ and CDF. They record the $p \bar{p}$ collisions at a center-of-mass energy of $1.96 \mathrm{TeV}$. The beam half-life-time is about 10 hours, and collisions are recorded for typically about 24 hours. After that period of time, the beams are dumped intentionally because of decreased beam focus and currents, resulting in an exponentially decreasing luminosity.

\subsection{The $\mathrm{D} \varnothing$ Detector}

The DØDetector is a multi-purpose detector that uses dedicated subsystems arranged cylindrically around the interaction point to identify and measure photons, electrons, muons and jets of quarks and gluons [55]. Figure 3.2 shows the upgraded D $\varnothing$ detector. The detector consist of three major subsystems, the central tracking system, the calorimeter system and the muon spectrometer. They will be described briefly in the following chapters.

\subsubsection{Tracking System}

The purpose of the tracking system is to measure the trajectories of charged particles produced in a collision. In addition, it is used to determine the primary interaction vertex 


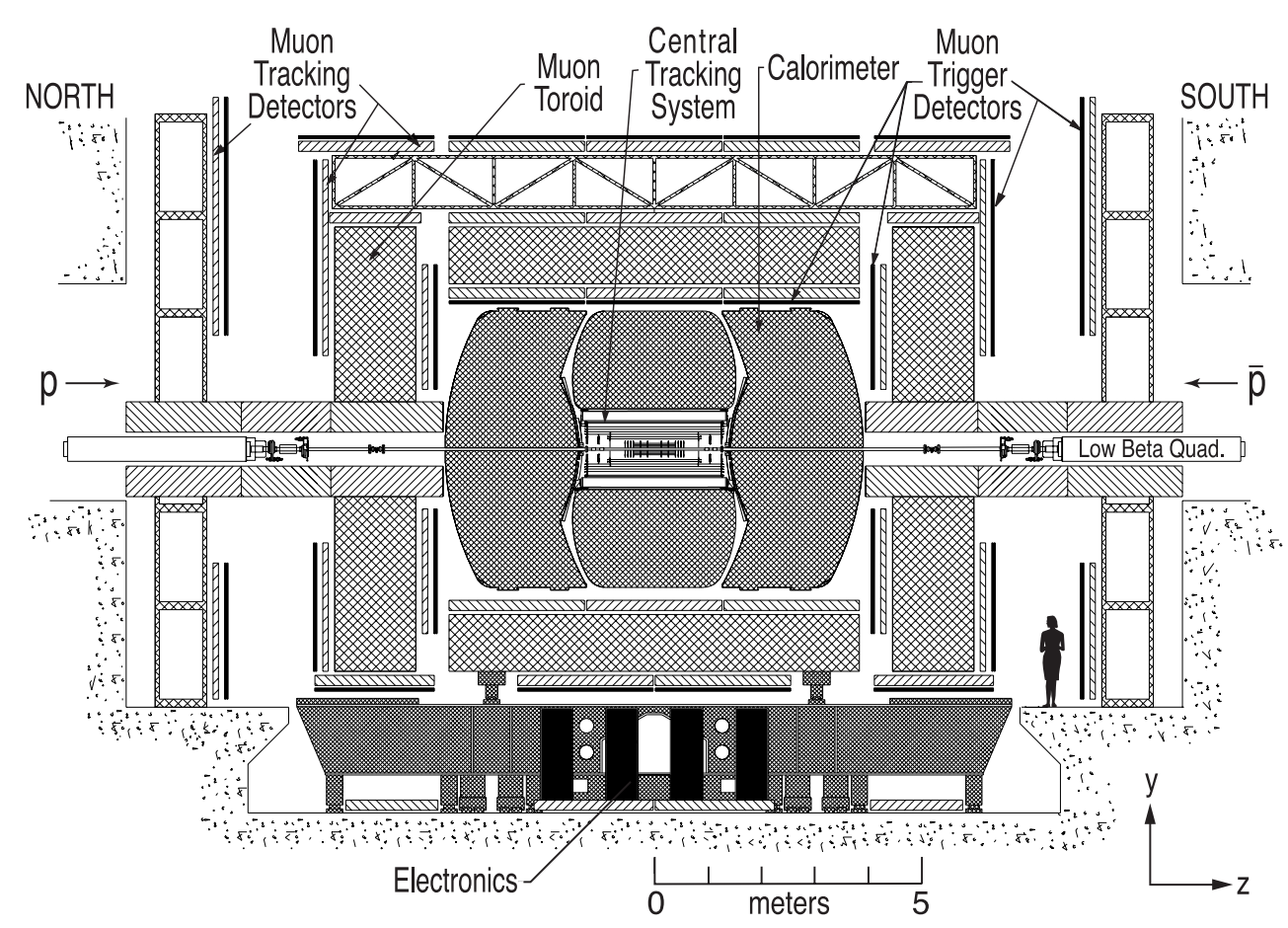

Figure 3.2.: Side view of the upgraded D $\varnothing$ detector (from Ref. [55]).

and decay vertices of long-lived particles such as B mesons. The D $\varnothing$ tracking system consists of the silicon microstrip tracker (SMT) and the central fiber tracker (CFT) in a $2 \mathrm{~T}$ solenoidal magnetic field [55]. Its performance is of vital importance for the analysis presented in this thesis, since the electron, muon and primary vertex reconstruction rely heavily on it. A schematic view of the tracking system is shown in Figure 3.3 .

\section{Silicon Microstrip Tracker (SMT)}

The SMT is the innermost tracking detector, starting at a radius of $2.7 \mathrm{~cm}$ [55]. The design was driven by the aim to cover the interaction region $(\sigma \approx 25 \mathrm{~cm})$ and to provide both tracking and vertexing over nearly the full $\eta$ coverage of the calorimeter and muon systems. Figure 3.4 provides an isometric view of the SMT. In the central region $(|z|<53$ $\mathrm{cm})$, the detector consists of six barrel modules; each barrel being capped at high $|z|$ with a disk of twelve double-sided wedge detectors ( F-disks). Each barrel module consist of four silicon readout layers. Layer 1 and 2 are equipped with 12 double-sided silicon readout modules (called ladder), layer 3 and 4 with 24 modules. Three F-disks are located forward of each barrel/disk assembly.

In the far forward region at high $|\eta|$, tracking is provided by two large-diameter disks (so-called $H$-disks). They are located 110 and $120 \mathrm{~cm}$ from the detector center. The barrel detectors primarily measure the $r-\phi$ coordinate, while the disk detectors measure $\mathrm{r}-\mathrm{z}$ as well as $r-\phi$. Hence vertices for high $\eta$ particles are reconstructed in three dimensions by the disks, and vertices of small- $\eta$ particles are measured in the barrels. 


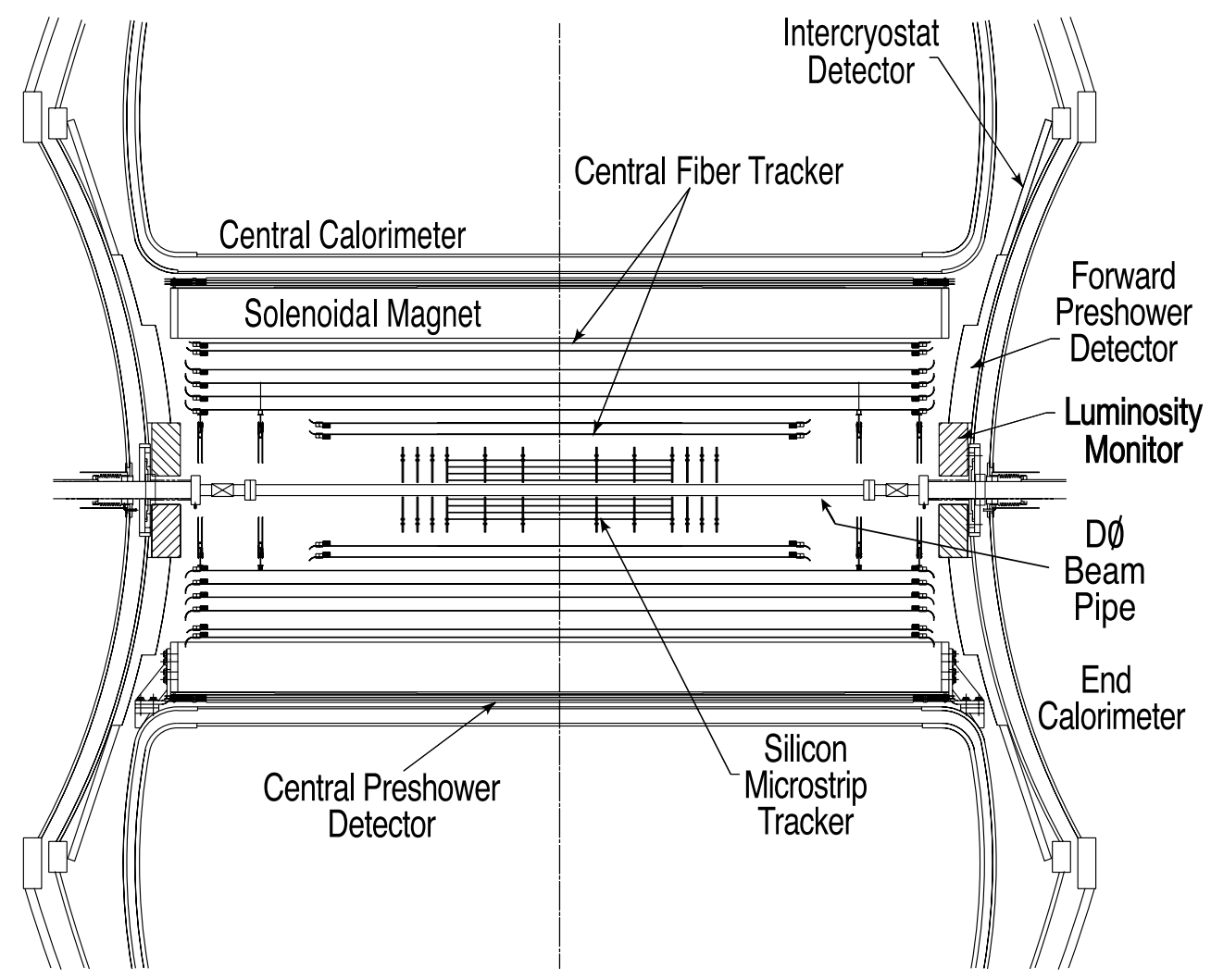

Figure 3.3.: Schematic view of the D $\varnothing$ tracking system (from Ref. [55]).

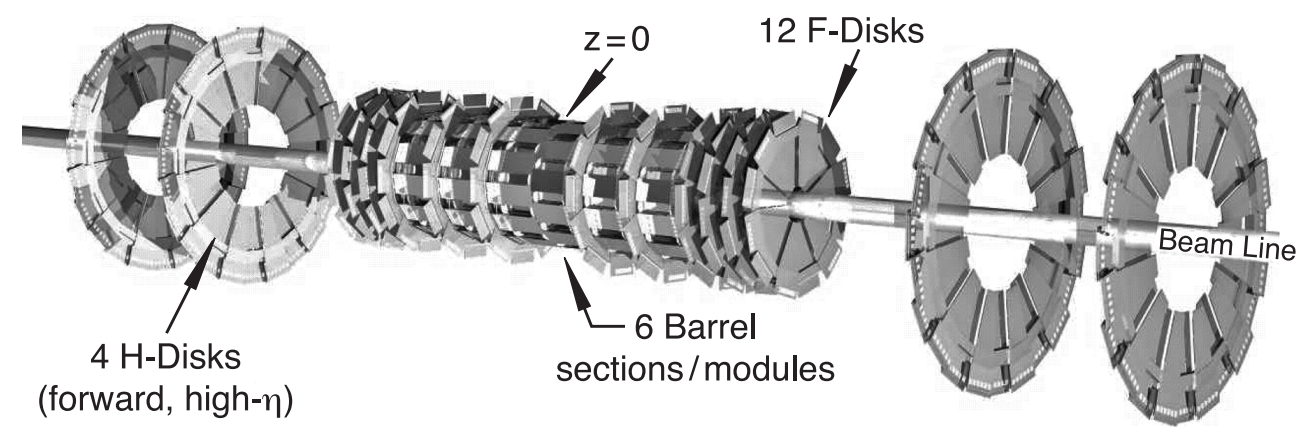

Figure 3.4.: The design of the Silicon Microstrip Tracker with its barrels and disks (from Ref. [55]). 


\section{Central Fiber Tracker (CFT)}

The CFT surrounds the SMT and provides tracking up to $\left|\eta_{\text {det }}\right|<1.6$ [55]. It consist of scintillating fibers mounted on eight concentric support cylinders. Each cylinder supports one doublet layer of fibers oriented along the beam direction(z-layer) and a second doublet layer at a stereo angle of +3 or -3 degrees ( $u$-layer or $v$-layer $)$. The scintillating fibers are either $1.66 \mathrm{~m}$ (two innermost cylinders) or $2.52 \mathrm{~m}$ (outer six cylinders) long and optically connected to clear fiber waveguides, which carry the scintillation light to visible light photon counters (VLPCs) for read out. From the smallest cylinder outward, the fiber layers are oriented zu-zv in alternating mode. The CFT has a total of 76,800 readout channels. Signals from z-layers are used to form a fast hardware trigger based upon the number of track candidates above a specific $p_{T}$ threshold.

\section{Solenoid Magnet}

The solenoid magnet surrounds the CFT at a radius of $60 \mathrm{~cm}$ and has a length of $2.7 \mathrm{~m}$ [55]. It provides a uniform magnetic field of $2 \mathrm{~T}$ inside the tracking volume and consist of two concentric coils of superconducting $\mathrm{Cu}: \mathrm{NbTi}$ cable. The magnet is operated at a temperature of $10 \mathrm{~K}$ with a current of $4.7 \mathrm{kA}$. The magnetic field stores an energy of $5 \mathrm{MJ}$. The superconducting solenoid coil and the cryostat walls have in total a thickness of about 1 electromagnetic interaction length $\left(X_{0}\right)$ at $\eta_{\text {det }}=0$.

\subsubsection{Preshower Detectors}

The preshower detectors operate as tracking detectors as well as calorimeters, improving the spatial matching resolution between electromagnetic showers in the calorimeters and tracks. Preshower information is also used in the trigger system due to the fast energy and position measurements.

The central preshower (CPS) detector is located between the solenoid and the central calorimeter and covers the region $|\eta|<1.3$. The forward preshower (FPS) detectors cover $1.5<|\eta|<2.5$ and are attached to the faces of the calorimeter endcaps. Both the CPS and FPS detectors are made of triangular strips of scintillators, which are arranged in a way that there is no dead space between the strips. The CPS detector consist of three concentric cylindrical layers of scintillator strips that are arranged in an axial- $u-v$ geometry. Each of the two FPS detectors (north and south) is made of two double layers of scintillating strips, which are separated by a $2 X_{0}$-thick lead-stainless steel absorber. The layers in front of the absorber are referred to as the minimum ionizing particle (MIP) layers, while the layers behind the absorber are called shower layers.

For the data used in the analysis presented, the preshower detectors were not fully included in both the triggering and the offline reconstruction. Because of this, their information is neglected.

\subsubsection{Calorimeter System}

The calorimeter provides energy measurement for electrons and photons in the form of clusters and for hadrons in the form of jets. By measuring shower characteristics like longitudinal profile, shower width and isolation it assists in the identification of electrons, 


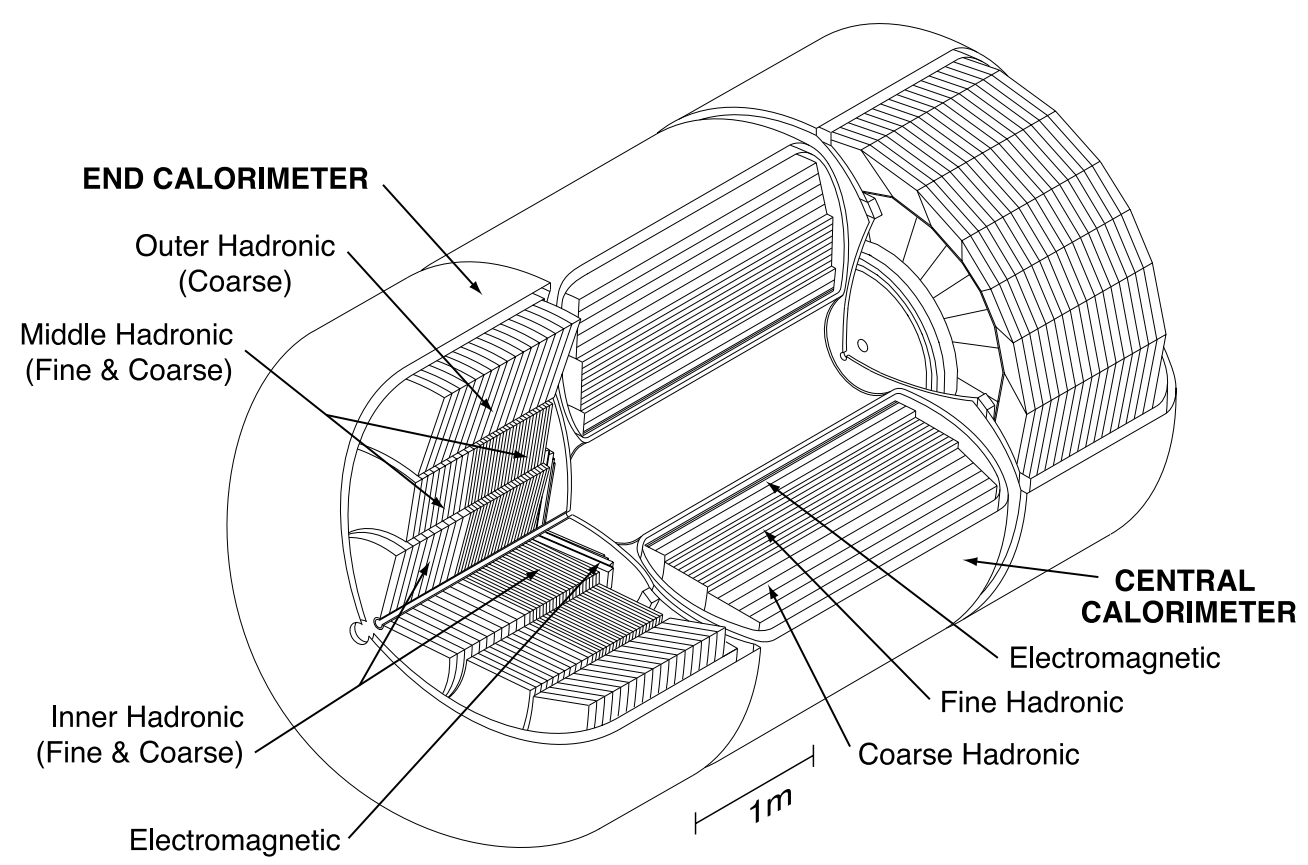

Figure 3.5.: Isometric view of the calorimeter system (from Ref. [55]).

muons, photons and jets. The $\mathrm{D} \varnothing$ calorimeter system consists of three uranium/liquidargon calorimeters and the intercryostat calorimeter, which are described briefly in the following.

\section{Liquid Argon Calorimeter}

The calorimeters are shown in Fig. 3.5. The central calorimeter $(C C)$ covers $\left|\eta_{\text {det }}\right|<1.1$, and the north and south endcap calorimeters $(E C N$ and $E C S)$ extend the $\eta$ coverage up to $\left|\eta_{\text {det }}\right| \lesssim 4$ [55]. Each calorimeter is located in a separate cryostat and contains an electromagnetic section closest to the interaction region followed by fine and coarse hadronic sections. The active medium for all the calorimeters is liquid argon that is kept at $80 \mathrm{~K}$ inside the cryostats. Calorimeter cells are the basic building blocks of the pseudo-projective towers shown in Fig. 3.6, and each tower is subdivided in depth. The towers in both the electromagnetic and hadronic part of the central calorimeter are $\Delta \eta \times \Delta \phi=0.1 \times 0.1$ except for the 3rd electromagnetic layer, where $\Delta \eta \times \Delta \phi=0.05 \times 0.05$. Cell boundaries lead to small insensitive regions in each layer, so-called $\phi$-cracks.

The electromagnetic section (EM) of the central calorimeter is subdivided in 4 depths called floors (EM1-EM4) representing $2+2+7+10$ radiation lengths at $\eta=0$. In the third electromagnetic floor, EM3, the calorimeter tower granularity is doubled in $\eta$ and $\phi$ because the maximum energy deposit of electromagnetic showers is expected. This leads to a precise measurement of the location and size of the shower.

The thickness of the hadronic section in the central calorimeter corresponds to about seven hadronic interaction lengths, divided into four floors (FH1-FH3 and $\mathrm{CH}$ ). The innermost floor, FH1, is included in the identification of electromagnetic objects since it allows to sample the energy deposition in the tail of the electromagnetic shower.

The electromagnetic section of the calorimeter endcaps as shown in Fig. 3.7 is built the 


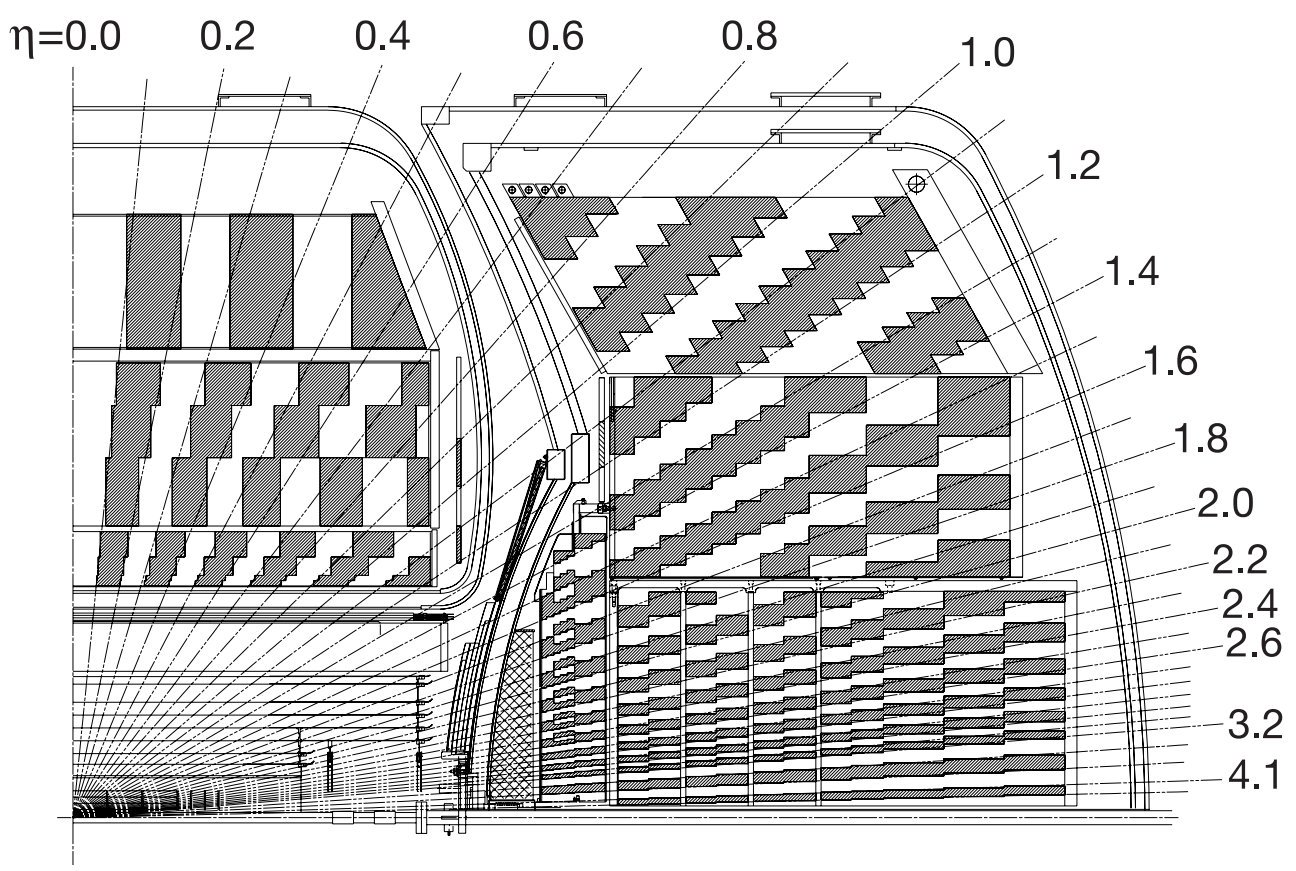

Figure 3.6.: Schematic view of a portion of the DØ central end endcap calorimeter showing the segmentation pattern. The lines indicate pseudorapidity intervals from the center of the detector (from Ref. [55]).

same way as the central part, but the floors represent now $0.3+2.6+8+9$ electromagnetic radiation lengths. Up to $|\eta|<2.6$, the cell granularity is the same as in the central calorimeter. To avoid very small cells, the cell size increases with $\eta$ up to a maximum value of $\Delta \eta \times \Delta \phi=0.4 \times 0.4$ at $\eta_{\text {det }} \approx 4.0$.

The hadronic section in the calorimeter endcaps is arranged into three modules. The two innermost hadronic modules are cylindrical in shape, consisting each of a fine and coarse hadronic part. The Inner Hadronic is composed of four fine hadronic and one coarse hadronic layers, with $4 \times 1.1+4.1$ hadronic interaction lengths. The Middle Hadronic consist of four fine hadronic and one coarse hadronic layer, too. However, their thickness is $4 \times 0.9+4.4$ hadronic interaction lengths. The Outer Hadronic modules have a maximum thickness of 6 hadronic interaction lengths.

In total, the calorimeter system has approximately 47,000 readout channels. The signals from each calorimeter cell are amplified and shaped in the preamplifiers, which are located on the cryostats, before being sent to the signal shaping and storage circuits that shape and sample them at their peak. To remove low frequency noise or pile-up, baseline subtraction is performed on the signal.

\section{Intercryostat Detector}

Between the central and the endcap calorimeter cryostats in the region $0.8<\left|\eta_{\text {det }}\right|<1.4$ the calorimeter coverage is incomplete. The gap between the cryostats is needed for supply lines and cabling of the central tracking system. There is a substantial amount of unsampled 


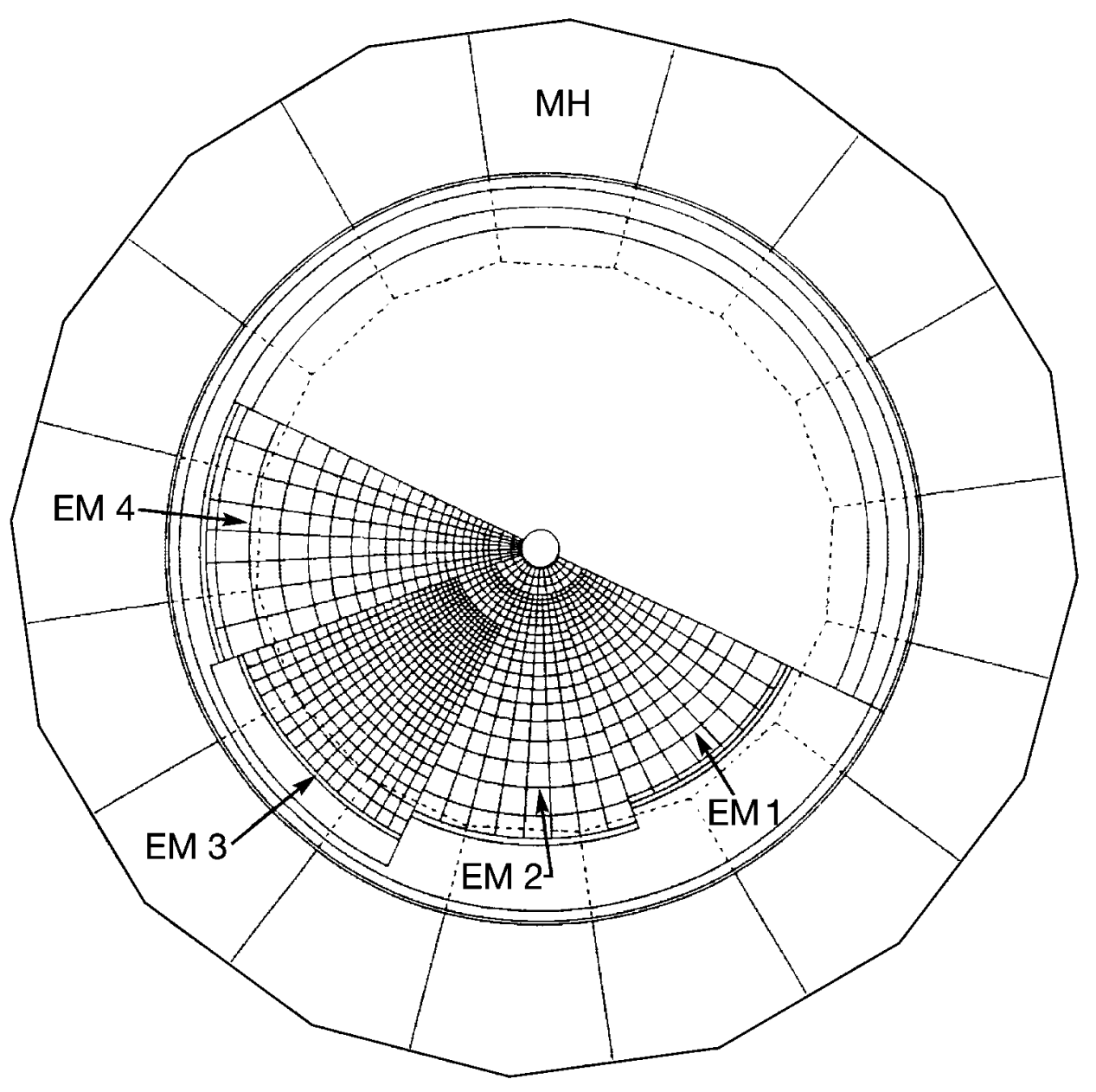

Figure 3.7.: Layout of the electromagnetic part of the endcap calorimeter. $M H$ indicates the middle hadronic section of the endcap. (from Ref. [55]). 
material in this region leading to energy resolution degradation. This is addressed by adding sampling in this region through single-cell structures without absorber. These so-called massless gaps are located inside the CC and EC cryostat walls. An additional inter-cryostat detector $(I C D)$ provided scintillator sampling in the region $1.1<\left|\eta_{\text {det }}\right|<1.4$, where no electromagnetic calorimeter is instrumented. The ICD is composed of a series of scintillating tiles, each covering $\Delta \eta \times \Delta \phi=0.3 \times 0.3$.

\subsubsection{Muon Spectrometer}

In contrast to other charged particles and hadrons, muons pass through the calorimeter leaving a minimum ionizing particle (MIP) like signal. Therefore, D $\varnothing$ uses a dedicated muon spectrometer to precisely measure and trigger on muons.

A muon signature consist of a signal in the muon system and a matching track from the tracking system in order to get a precise momentum measurement.

The muon spectrometer is the outermost part of the $\mathrm{D} \varnothing$ detector and like the calorimeter system, it is separated into central and forward regions. The central part is located at a distance of $3.18 \mathrm{~m}<\mathrm{r}<4.27 \mathrm{~m}$ to the beam pipe, and in the forward region $4.54 \mathrm{~m}<$ $|\mathrm{z}|<6.10 \mathrm{~m}$ from the interaction point. The toroid magnet creates an internal field of $1.8 \mathrm{~T}$ to allow momentum measurement in the muon system.

Figure 3.8 shows a schematic view of the DØ muon spectrometer. Its central part covers up to $\left|\eta_{\text {det }}\right| \leq 1$ and the forward part extends this to $\left|\eta_{\text {det }}\right| \leq 2$. Each part uses fast scintillation counters for triggering and timing measurements and drift tubes for precise position measurements, a rough momentum estimate and also for triggering.

The central region uses proportional drift tubes (PDTs) as drift chambers located in three layers inside (layer A) and outside (layers B and C) of the central toroid. The drift tubes are made of rectangular aluminum tubes of $10.1 \mathrm{~cm}$ across and a maximum length of $5.79 \mathrm{~m}$, each containing an anode wire at the center and cathode pads above and below the wire to provide hit information along the wire. The drift tube wires are operated at $4.7 \mathrm{kV}$ and the pads are operated at $2.3 \mathrm{kV}$. The gas mixture consists of $84 \%$ argon, $8 \%$ methane and $8 \% \mathrm{CF}_{4}$. The PDTs are arranged to chambers of three to four decks of drift tubes with 24 tubes each. The central muon system is only partially instrumented at the bottom region $(4.25<\phi<5.15)$ to allow for support structures.

The forward region uses mini drift tubes $(M D T \mathrm{~s})$ as drift chambers due to their short electron drift time ( less than $132 \mathrm{~ns}$ ), high segmentation and radiation hardness. The MDTs are arranged in A, B and C layers the same way the PDTs are. A layer consist of three to four planes of tubes, each tube comprises eight $1 \times 1 \mathrm{~cm}^{2}$ cells with a maximum length of $5.83 \mathrm{~m}$ operated at $3.2 \mathrm{kV}$. The MDT system uses a 90\%-10\% gas mixture of $\mathrm{CF}_{4}-\mathrm{CH}_{4}$.

Shielding structures near the beam pipe isolate the detectors from backgrounds. The scintillation counters in both the central and forward region are positioned alongside the PDTs and MDTs.

\subsubsection{Luminosity Monitor}

The purpose of the luminosity monitor is to make an accurate measurement of the Tevatron luminosity at the $\mathrm{D} \varnothing$ interaction region by measuring the rate of inelastic $p \bar{p}$ collisions. In addition, it measures beam halo rates, identifies beam crossings with multiple interactions 


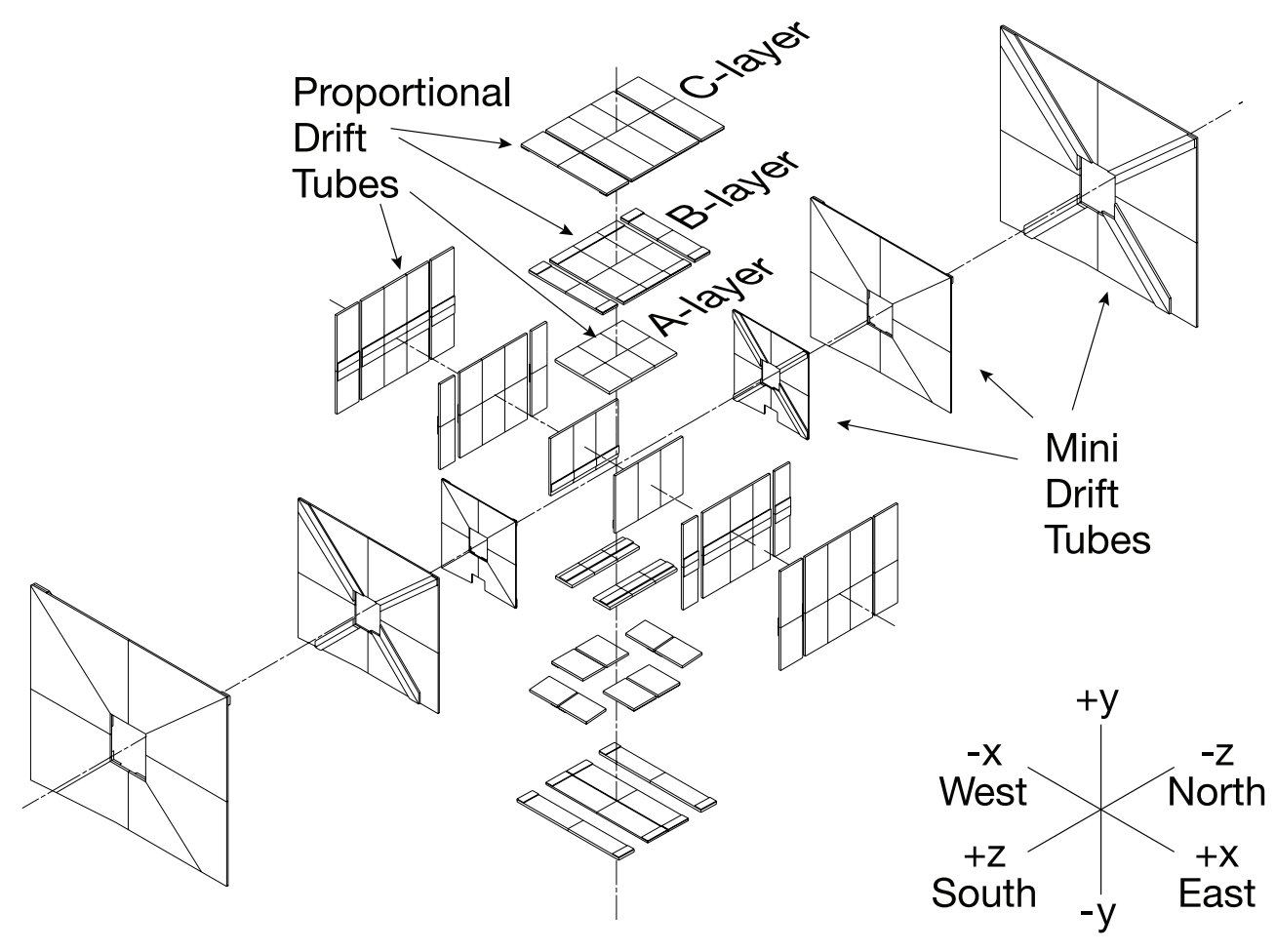

Figure 3.8.: Exploded view of the muon wire chambers (from Ref. [55]).

and makes a quick measurement of the z-coordinate of the interaction vertex. The luminosity monitor is placed in front of the calorimeter endcaps. Each detector consists of two arrays of 24 plastic scintillation counters with photomultiplier readout.

The luminosity $\mathcal{L}$ is determined from the number of inelastic collisions per beam crossing $\bar{N}_{L M}$ calculated with Poisson statistics from the number of beam crossings with no collisions measured by the luminosity monitor. With the beam crossing frequency $f$ and the effective cross section $\sigma_{L M}$ corrected for the acceptance and efficiency of the luminosity monitor, the luminosity $\mathcal{L}$ is given by $\mathcal{L}=\frac{f \bar{N}_{L M}}{\sigma_{L M}}$ The fundamental unit of time for the integrated luminosity measurement is called luminosity block and is indexed by the luminosity block number $(L B N)$ which increases monotonically throughout Run II. A luminosity block has a maximum length of $60 \mathrm{~s}$ which is short enough to assume a constant instantaneous luminosity.

\subsubsection{Forward Proton Detector}

The forward proton detector measures protons and antiprotons scattered at small angles that are missed by the $\mathrm{D} \varnothing$ detector. In addition to position detectors along the beam line, it makes use of the accelerator magnets. The position detectors are housed in stainless steel containers (roman pots), allowing them to function outside the accelerator's vacuum and to be moved away from the beam during unstable beam conditions. The forward proton detector consists of 18 roman pots arranged in six steel chambers located at various distances from the $\mathrm{D} \varnothing$ interaction point. 


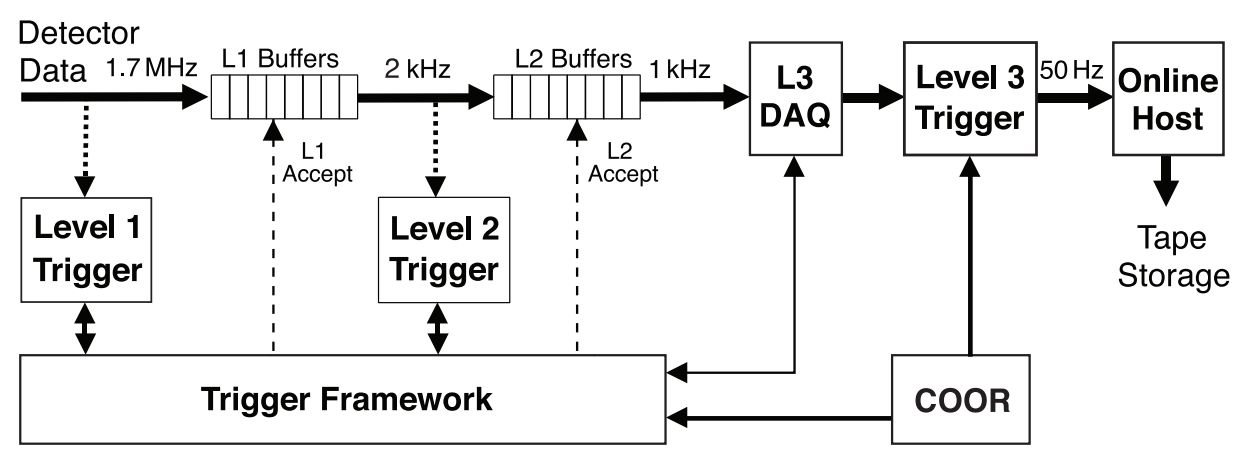

Figure 3.9.: Overview of the DØ trigger and data acquisition system (from Ref. [55]) .

\subsubsection{Trigger and Data Acquisition}

Most of the $p \bar{p}$ interactions are soft collisions and only of minor interest. Therefore, D $\varnothing$ uses a sophisticated trigger system to select the interesting physics events and to reduce the data flow from approximately $2 \mathrm{MHz}$ to a recordable rate of $50 \mathrm{~Hz}$. Three succeeding levels form the trigger system, each of them examining fewer events but in greater detail and with more complexity.

Figure 3.9 shows a schematic overview of the DØ trigger and data acquisition system. The triggers are configured by a so-called trigger list, a set of individual triggers which are defined by a set of requirements at each trigger level. The following sections provide a brief overview of the tasks and structure of each trigger level. A block diagram of the DØ trigger system is shown in Figure 3.10 .

\section{Level 1 Trigger}

The first trigger stage (Level 1 or L1) is implemented in custom-designed hardware to allow examination of every event for interesting signatures. A L1 trigger decision is made within $4.2 \mu \mathrm{s}$, resulting in a reduction of the data flow from $2 \mathrm{MHz}$ to $1.6 \mathrm{kHz}$. The L1 trigger uses the following detector subsystems to reach a trigger decision (see Fig. 3.10): calorimeter system $(L 1 C A L)$, central fiber tracker and central/forward preshowers ( $L 1 C T T)$, the muon system $(L 1 M U O)$ and the forward proton detector (L1FPD).

\section{Level 2 Trigger}

The second trigger level (Level 2 or L2) collects data from both the front-end electronics and the L1 trigger processors in order to perform a more detailed event analysis. L2 can combine data across detectors to form higher quality physics objects and to examine eventwide correlations in all L2 physics objects. The system can handle a maximum input rate of $10 \mathrm{kHz}$ and has an accept rate of $1 \mathrm{kHz}$ at maximum. Its maximal latency is $100 \mu \mathrm{s}$, and the dead-time is $5 \%$ at maximum.

The L2 trigger consists of preprocessors for each detector subsystem and a global processor that integrates the data. The preprocessor subsystems include tracking, calorimeter, preshower and muon detectors. All subsystems work in parallel, and the global L2 processor makes the final trigger decision based on physics objects reconstructed in the preprocessors. 


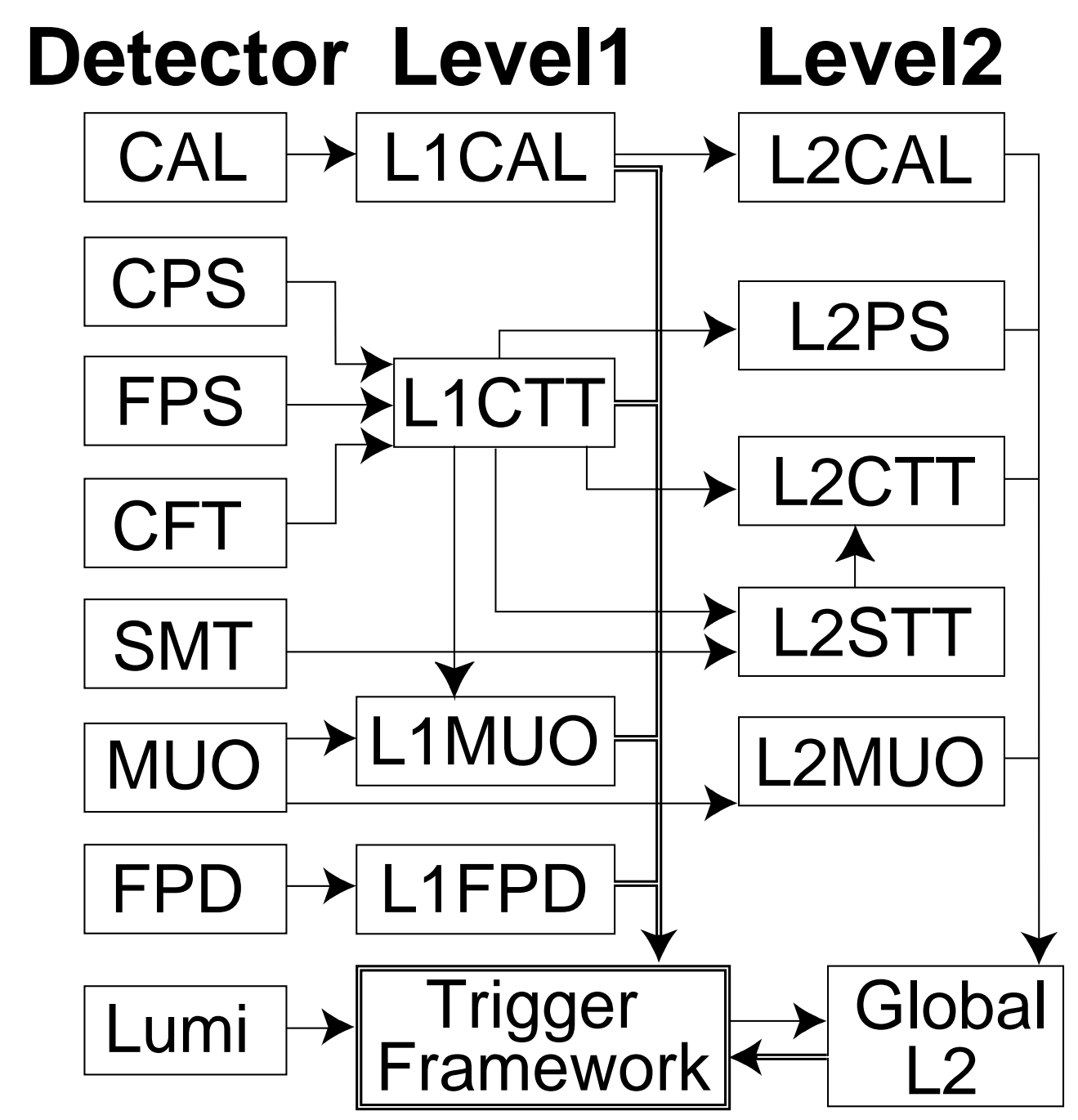

Figure 3.10.: Diagram of the Level 1 and Level 2 trigger systems. The data flow is indicated by the arrows. (from Ref. [55]). 


\section{Level 3 Trigger}

For Events passing the L1 and L2 triggers, the entire D $\varnothing$ detector is read out including the L1 and L2 systems themselves. Each event is reconstructed fully with algorithms running on a CPU farm that resemble the offline reconstruction as closely as possible while taking restrictions due to processing power into account. This allows Level 3 to make a final trigger decision within $200 \mathrm{~ms}$, reducing the maximum input rate from $1 \mathrm{kHz}$ to an output rate of $50 \mathrm{~Hz}$. The decisions of the L3 trigger are based on complete physics objects as well as on relations between them, e.g. spatial separation of physics objects. Candidate physics objects are reconstructed by object specific software algorithms (so-called filter tools). Reference sets of algorithm parameters that define the physics objects are input to the filter tools via the trigger list.

\section{Data Acquisition System}

The data acquisition system of the $\mathrm{D} \varnothing$ detector consists of the $L 3 D A Q$ and the online host system. The task of the former is to transport detector data stored in readout crates to processing nodes of the L3 trigger farm. The online host system receives data from the L3 farm nodes and distributes it to logging and monitoring tasks.

The raw data is written to files and transported to the mass storage system consisting of disk and tape storage. Corresponding meta data is created and stored in a database. The raw data is reconstructed with the DØRECO software package, which provides two output formats, the data summary tier (DST) and the thumbnail (TMB). The DST contains all information necessary to perform any physics analysis, including re-reconstruction of highlevel physics objects. Its size corresponds to about $150 \mathrm{kB}$ per event, while the TMB size is approximately $20 \mathrm{kB}$ per event. The TMB can be used directly to perform many physics analyses. The size reduction compared to the DST is achieved by compression and dropping part of the information. 


\section{Event Reconstruction}

The purpose of the event reconstruction and object identification is to translate the stream of readout signals from the detector into basic physics objects. Elaborate algorithms have been developed to identify tracks, primary and secondary vertices, electrons, muons, taus, jets and neutrinos (in the form of missing transverse energy, $\mathbb{E}_{T}$ ) as precisely as possible, calibrate and correct them in order to reconstruct the event kinematics.

The following chapter discusses the reconstruction of physics objects which are relevant to this analysis. The event signature consists of two muons, two jets and missing transverse energy. The reconstruction of these objects is described in detail. Additional information is needed from tracks and the primary vertex, which will also be addressed, as well as strategies for separating these physics objects from background.

\subsection{Track Reconstruction}

A charged particle trajectory (called track) is reconstructed using hit information in the tracking detectors, SMT and CFT (see Section 3.2.1). Both detectors are used for reconstructing tracks in $\left|\eta_{\text {det }}\right| \leq 1.6$, while only the SMT can be used for tracking up to $\left|\eta_{\text {det }}\right| \lesssim 4$. The track reconstruction relies on two algorithms: the Histogram Track Finder [56] $(H T F)$ and the Alternative Algorithm [57] $(A A)$.

The HTF method relies on a histogramming method. The trajectory of a charged particle moving perpendicular to a homogeneous magnetic field can be characterized by the curvature radius $\rho$, the distance of closest approach with respect to the beam spot $(0,0)$ $d_{0}$ and the azimuth direction of the track at the distance of closest approach to $(0,0), \phi$. Every pair of hits in $\mathrm{x}$ and $\mathrm{y}$ that belongs to the same track candidate corresponds to a single point in the $\rho-\phi$ plane for track candidates with small $d_{0}$. Thus filling each pair of hits into the two-dimensional $\rho-\phi$ histogram results in a distribution with several peaks, where each peak corresponds to a track candidate.

The AA uses a road-following method to reconstruct tracks. The algorithm extrapolates from any three-hit combination in the SMT barrels or disks the sequence of hits moving outward to the next SMT or CFT layer. A $\chi^{2}$ test is performed if a hit is found within the search window and the hit is associated with the track candidate if the $\chi^{2}$ value is below a certain threshold. A "miss" is recorded when no hit is found in the layer. The algorithm stops when the last CFT layer is reached or three misses are recorded.

A final list of tracks is generated by merging the output of the AA and HTF algorithms and removing duplicate track candidates. The transverse momentum $p_{T}$ of a track is calculated from the curvature radius in the magnetic field. The momentum resolution degrades with increasing momentum as the curvature radius increases. In addition, the $p_{T}$ measurement for electrons and muons suffers from bremsstrahlung radiation. 


\subsection{Vertexing}

The vertex of the hard scattering event is called primary vertex. The measurement of its position is crucial for the accurate measurement of the physics objects' $\eta$ and their transverse momentum. Additional (soft) minimum-bias interactions or a second hard scattering process in the same bunch crossing, especially at high instantaneous luminosities, can lead to more than one reconstructed primary vertex as well as misreconstruction can. Additionally, decay vertices of heavy quark decays (so-called secondary vertices) are reconstructed.

Primary vertex candidates are determined using reconstructed tracks [58]. At least three tracks with SMT hits have to point to the same vertex. Its exact position is determined by a fit that uses the associated tracks. The vertex z position has to be within $|z|<60 \mathrm{~cm}$, which is the SMT acceptance region. Based on track multiplicity and the transverse momentum of the associated tracks, the primary vertex is picked among the vertex candidates.

\subsection{Muon Reconstruction and Identification}

Muons are reconstructed based on hits in the central and forward muon drift chambers and scintillators within $\left|\eta_{\text {det }}\right|<2.0$ and tracks reconstructed in the central tracking system [59]. Muon reconstruction starts with the formation of so-called "stubs", straight-line segments in the muon system that contain both a muon wire chamber and a scintillator hit. Stubs can be reconstructed on either side of the toroid in layers A or BC. If A-layer stubs can be matched to BC layer stubs, taking into account the trajectory bend in the $1.8 \mathrm{~T}$ magnetic field and multiple scattering caused by the muon's passage through the iron toroid, the stubs are combined to form a "local" muon. Local muons can be paired with tracks by matching the track direction with the muon direction as measured at the inner surface of the muon spectrometer, and matching the track momentum with the muon momentum as measured independently in the muon spectrometer from the bend angle through the toroid.

Although there is no precise momentum measurement or no momentum measurement at all from single-layer stubs, they can still be matched to central tracks. As there is no momentum measurement for A-layer stubs, they are matched to central tracks based only on direction. BC-layer stubs are matched using direction and an estimate of the muon momentum calculated by assuming the muons originated at the primary vertex.

A reconstructed muon consists of either a local muon matched to a central track or a stub-track pair. A veto on cosmic muons is applied by requiring the time difference between scintillator hits in B or C layer (subsequently referred to as "BC-layer") and the A layer to be consistent with a muon coming from the interaction region. The muon tracks are then extended to the point of closest approach $(P C A)$ to the beam and their parameters are compared to those of central tracks at the point of closest approach. A fit is performed with all central tracks within one radian in azimuthal and polar angle of a muon track at PCA. The central track with the lowest $\chi^{2}$ is considered as the muon track.

\subsubsection{Muon Type and Quality}

The kind of muon is determined by two parameters, the muon "quality" and the muon "type" [60]. The type of a muon candidate is described by a parameter called "nseg". A positive nseg value indicates that the local muon was matched to a central track. A 


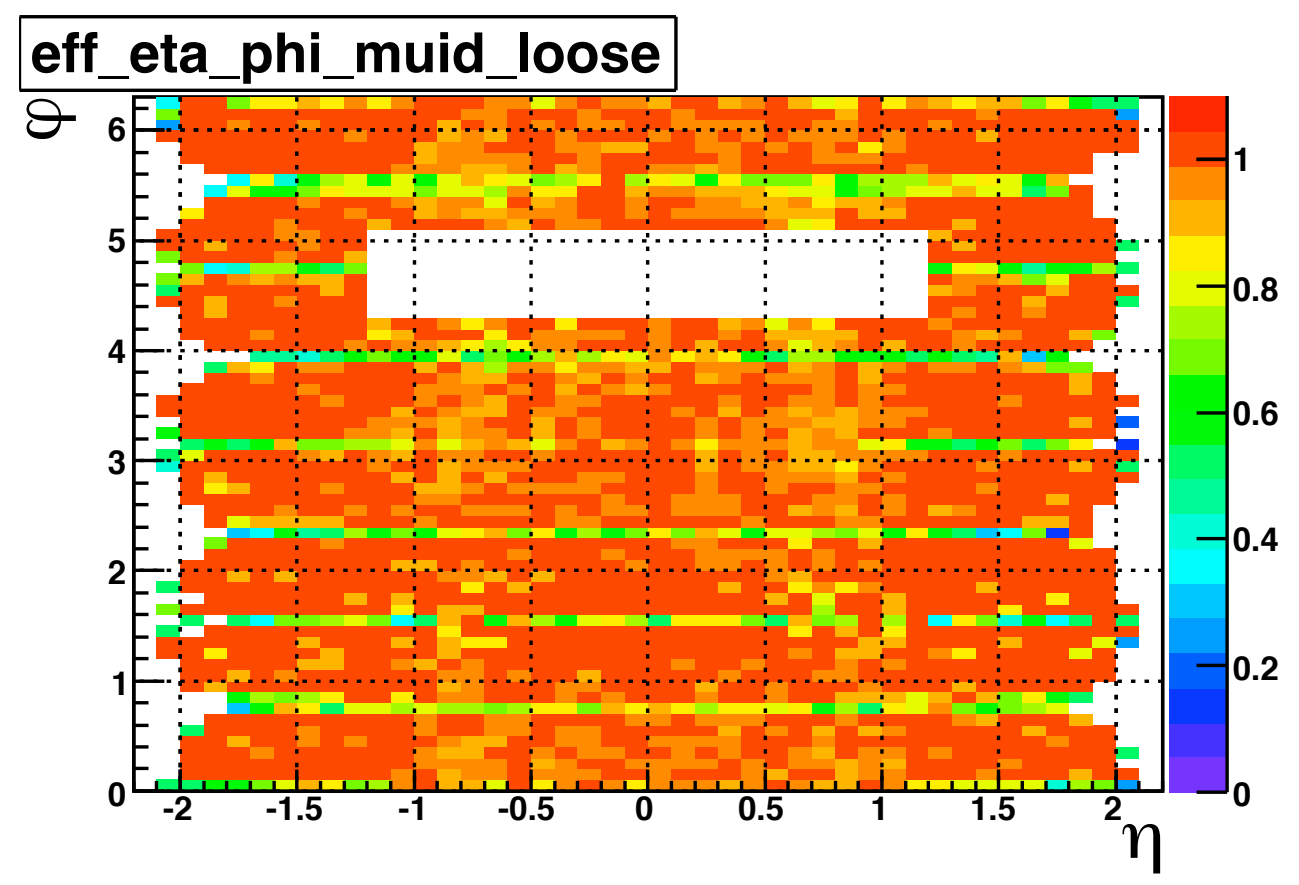

Figure 4.1.: Muon identification efficiency in the $\eta_{d e t}-\phi$ plane for muons with "loose" quality, as measured in data (taken from [59]). Muons identified in the "bottom hole" where the detector feet are, are rejected.

negative value of nseg tells that the muon could not be matched to a track in the central tracking system, respectively.

The absolute value of nseg indicates that the local muon is made up of A-layer only hits (nseg=1), BC layer hits (nseg=2) or both A-layer and BC-layer hits (nseg=3). The muon quality can be "Loose", "Medium" or "Tight". The exact definition is dependent on the value of nseg [59].

For loose muons with $n s e g=1$ at least one scintillator hit and two A layer wire hits are required, while loose quality muons with $n s e g=2$ have to have one scintillator hit in either $\mathrm{B}$ or $\mathrm{C}$ layer, and two wire hits in either the $\mathrm{BC}$ layer. For both $n s e g=1$ and $n s e g=2$, medium quality muons are defined as loose muons located in the two bottom octants of the detector, and with $|\eta|<1.6$.

A muon is of tight quality if $|n s e g|=3$ and it has at least two A layer wire hits and one A layer scintillator hit, at least three wire hits in the BC layer and one B layer scintillator hit. Moreover, the track stubs in the A and BC layer could be combined into a local muon.

Muons with $\mid$ nseg $\mid=3$ can still be of loose or medium quality when they fail the criteria for tight muon quality. Medium quality muons with $\mid$ nseg $\mid=3$ are required to have at least two A layer wire hits, one A layer scintillator hit, two BC layer wire hits and at least one $\mathrm{BC}$ scintillator hit if the muon is central and has less than four BC layer hits. When failing one of these requirements (where the A layer wire and scintillator requirement count as one), the muons are of quality loose. Still, loose $\mid n$ seg $\mid=3$ muons have to have at least one scintillator hit. Fig. 4.1 shows the reconstruction and identification efficiency for muons of type "Loose" with nseg $\geq 0$. 

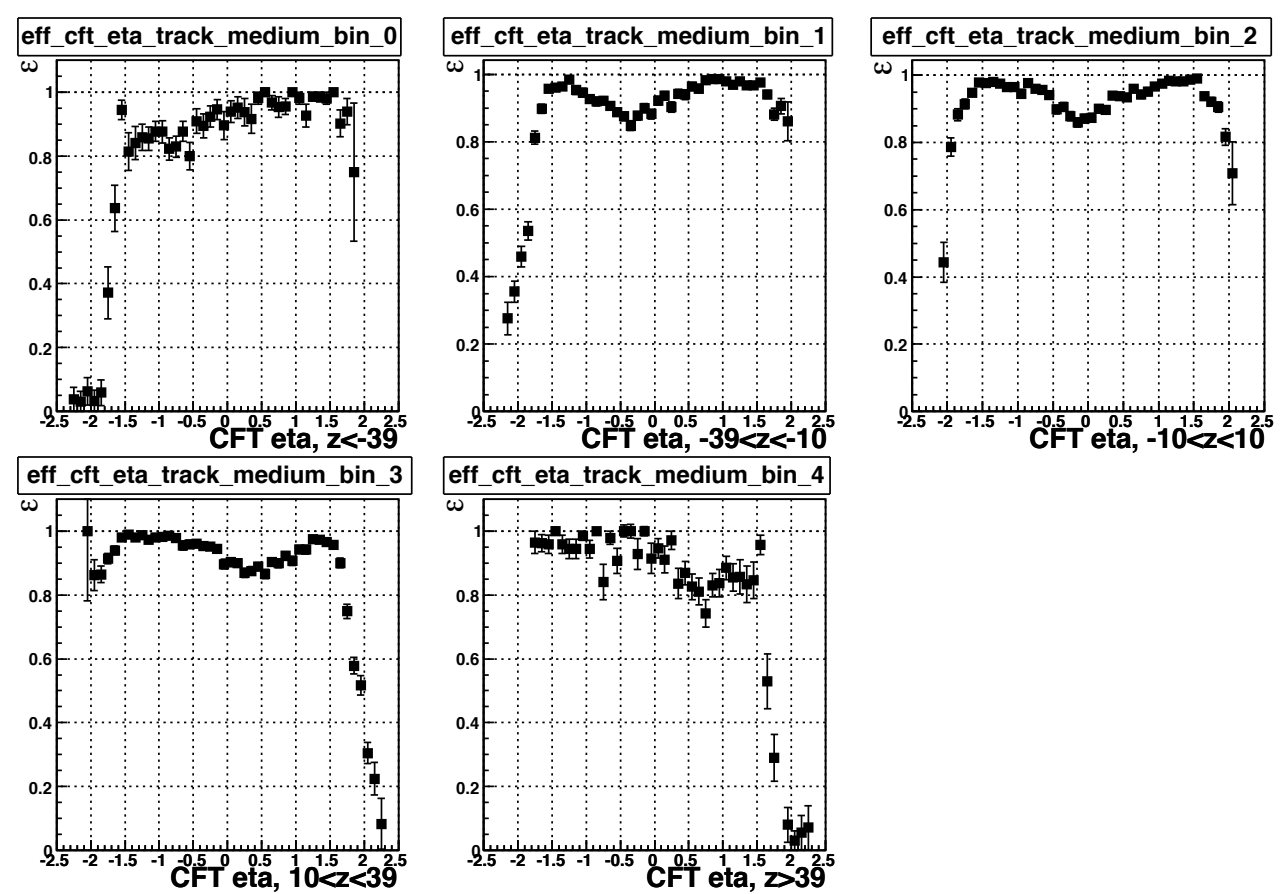

Figure 4.2.: Efficiency for a "medium" track match as a function of $\eta_{C F T}$, as measured in data (taken from [59]). The efficiency is shown for for different ranges of track $\mathrm{z}$ values.

\subsubsection{Central Track Quality Definitions}

Three track qualities have been defined in order to control the purity of the muons matched to a central track. These definitions rely on the number of hits in the SMT and CFT, the distance of closest approach $d c a$ of the track with respect to the primary vertex of the event and the $\chi^{2}$ per degrees of freedom $\left(N_{d o f}\right)$ of the central track fit. A "loose" track has no cut on $\chi^{2} / N_{d o f}$, is not required to have any SMT hits and $|d c a|<0.2 \mathrm{~cm}$. The cut on $d c a$ is tightened to $0.02 \mathrm{~cm}$ if the track has SMT hits. In addition, a "medium" track has $c h i^{2} / N d o f<4$, and a "tight" track fulfills the "medium" track match requirements and has at least one SMT hit. The track matching efficiency as a function of $\eta_{C F T}$ in different primary vertex $\mathrm{z}$ bins is shown in Fig. 4.2 .

\subsubsection{Muon Isolation}

Muon isolation variables are used to separate isolated muon from e.g. $W \rightarrow \mu \nu$ from heavy flavor background $(B \rightarrow \mu+X)$. Because muons from heavy flavor decays tend to be inside a jet, these variables are either defined in terms of tracks near the muon track or calorimeter energy surrounding the muon momentum vector. In this analysis, we use the following isolation variables:

- The distance to the closest jet, $\Delta R(\mu$, jet $)$

- The sum of the transverse momentum of all tracks within a 0.5 cone around the muon track excluding the muon track, divided by the muon's $p_{T}:\left|\sum_{\text {tracks }} p_{T} / p_{T}(\mu)\right|$ where $\Delta R($ track, muontrack $)<0.5$ 


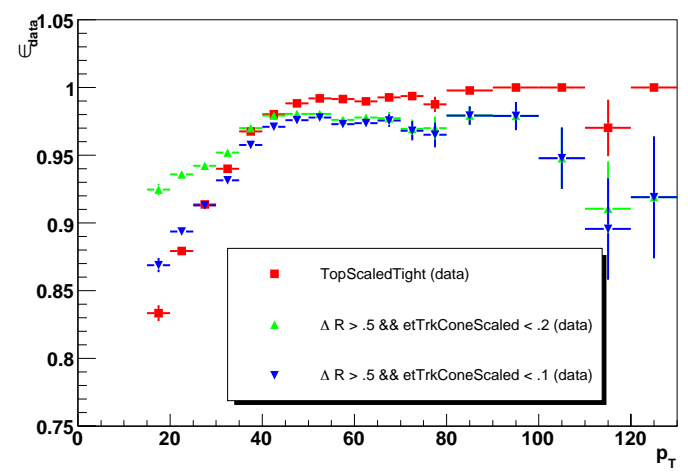

Figure 4.3.: Efficiency of muon isolation criteria versus $p_{T}$ measured in data.

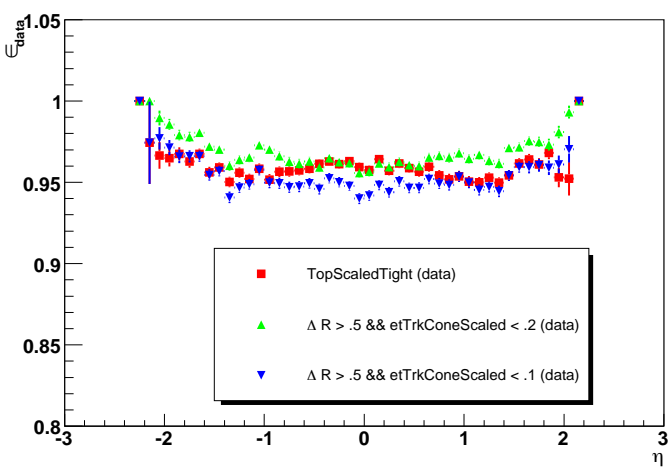

Figure 4.4.: Efficiency of muon isolation criteria versus $\eta$ measured in data.

The efficiency defined by $\Delta R(\mu$, jet $)<0.5$ and either $p_{T}:\left|\sum_{\text {tracks }} p_{T} / p_{T}(\mu)\right|<0.1$ or $p_{T}$ : $\left|\sum_{\text {tracks }} p_{T} / p_{T}(\mu)\right|<0.2$ as a function of transverse muon momentum and $\eta_{C F T}$ of the muon track is shown in Figs. 4.3 and 4.4. It is compared to an isolation definition termed "TopScaledTight" that uses the energy deposition in the calorimeter around a hollow cone of the muon track in addition to the tracker isolation defined above.

\subsection{Electromagnetic Object Reconstruction}

Because electrons are identified in the calorimeter, their reconstruction is similar to jets. The reconstruction software uses calorimeter towers in the first five calorimeter layers as an input to the simple cone algorithm [61] that forms clusters with a cone size of $\Delta R=$ 0.4 .

Since the reconstructed electromagnetic clusters are dominated by background from hadronic jets, further requirements have to be imposed on the clusters. These requirements are based on the shape differences between electromagnetic and hadronic showers. The latter are broad with a large fraction of energy deposited in the hadronic section of the calorimeter. In addition to a minimum transverse energy of $1.5 \mathrm{GeV}$ and an electromagnetic fraction (EMfrac) of 0.9 , it is required that the isolation of the electromagnetic cluster is less than 0.2. The isolation (iso) is defined as

$$
i s o=\frac{E_{t o t}(0.4)-E_{E M}(0.2)}{E_{E M}(0.2)}
$$

where $E_{t o t}(\Delta R)$ and $E_{E M}(\Delta R)$ denote the total energy and electromagnetic energy within a cone of radius $\Delta R$ [62].

The energy of the electromagnetic cluster corresponds to the sum of the energy deposited in all five layers used in the cone algorithm. A calibration is performed that has been derived by the invariant dielectron mass and its resolution in $J / \Psi \rightarrow e e$ and $Z \rightarrow e e$ events.

Since photons do not leave signals in the central tracking system, requiring that a track is matched to the electromagnetic cluster is a powerful way to draw a distinction between electrons and photons. The analyses presented here utilize the so-called $E / p$ track match to associate a central track to an EM cluster. The algorithm searches within a window 
of $\Delta R<0.1$ around the calorimeter cluster center for candidate tracks. Since the track momentum is expected to match the transverse energy measurement in the calorimeter, the $\chi^{2}$ has in addition to $\Delta z$ and $\Delta \phi$ terms a piece using the ratio of the track momentum and EM cluster transverse energy:

$$
\chi^{2}=\left(\frac{\Delta z}{\sigma_{z}}\right)^{2}+\left(\frac{\Delta \phi}{\sigma_{p h i}}\right)^{2}+\left(\frac{\frac{E_{T}}{p_{T}}-1}{\sigma_{E_{T} / p_{T}}}\right)^{2}
$$

In addition to these basic identification criteria, a shower shape variable is defined that tests the consistency of the EM cluster shower with the hypothesis of an electron shower. This $\chi^{2}$ variable is termed $H M x \eta$ because it is an H-Matrix discriminant built out of seven variables The H-Matrix approach was introduced by Fisher [63] and Mahalanobis [64]. The discriminator is defined as

$$
\chi_{U}^{2}=\sum_{k, l=1}^{n_{\text {var }}}\left(x_{k}-\bar{x}_{U, k}\right) C_{U, k l}^{-1}\left(x_{l}-\bar{x}_{U, l}\right)
$$

where $x_{i}$ denotes the $n_{v a r}$ variables used to distinguish signal from background and $\mathrm{U}=\mathrm{S}$, B. The covariance matrices $C_{U, k l}$ and the sample means $\bar{x}_{U, k}$ have to be obtained from training samples.

Finally, an electron likelihood is defined based upon seven variables including the electromagnetic fraction, the quality of the track match and $E_{T} / p_{T}$.

\subsection{Jet Reconstruction and Identification}

Collimated showers of hadrons are observed as jets in the detector, since individual quarks and gluons cannot be directly detected due to color confinement.

The jet algorithm uses the simple cone algorithm to find proto-clusters that serve as seed clusters to the Run II Cone Algorithm. The Run II Cone Algorithm consists of three steps, clustering, addition of midpoints and merging/splitting of clusters [65] [66].

This analysis uses $\Delta \mathrm{R}=0.5$ cone jets, which have to fulfill the following identification requirements [67]:

- $E M F<0.95$ : The fraction of the jet deposited in the electromagnetic part of the calorimeter $(E M F)$ is used to remove isolated electromagnetic particles (electrons or photons).

- $C H F<0.4$ : The calorimeter noise from cells is dominated by the coarse hadronic layers. To remove jets which are faked by noise in the coarse hadronic layers, the coarse hadronic energy fraction $(C H F)$ has to be smaller than 0.4.

- To further remove jets originating from noise in the calorimeter readout, the ratio of the jet energy measured by the L1 system to the precision readout jet energy must be larger than 0.4. This is referred to as "L1 confirmation".

A jet that satisfies the above criteria is regarded as a "good" jet. 


\subsubsection{Separation of Jets and Electromagnetic Objects}

Electrons and photons with a transverse energy greater than $8 \mathrm{GeV}$ are also reconstructed as jets in the calorimeter. This presents the problem of differentiating properly between real jets and electrons, and applying the appropriate energy correction. In this analysis, jets overlapping with a high $p_{T}$ electron (selected following the electromagnetic object identification based on electromagnetic fraction, isolation, shower shape and track match) are removed from the set of good jets.

\subsubsection{Jet Energy Scale Correction}

The goal of the jet energy scale correction is to correct the reconstructed jet energy back to the stable-particle jet level before interacting with the detector. To do so, the reconstructed jet energy has to be corrected for a number of detector effects [68]:

- Energy Offset $(\mathrm{O})$ : Energy contribution to the reconstructed jet from the underlying event, multiple interactions, pile-up, electronics noise and noise from the uranium absorber can result in an offset to the jet energy (Fig. 4.5). It is computed by adding up the estimated energy density from all calorimeter towers within the jet cone. The per-tower energy density is measured in minimum-bias events (events triggered by the luminosity monitor) as a function of the primary vertex multiplicity to account for the instantaneous luminosity dependence.

- Relative Response Correction $\left(\mathrm{F}_{\eta}\right)$ : The goal of this correction is to make the calorimeter response uniform over the whole pseudorapidity range. While the calorimeter is quite uniform in the central and endcap cryostats, the gap between them is not as well instrumented, causing a non-uniform response as a function of $\eta$. The relative response correction is measured using the Missing Transverse Energy Projection Fraction $(M P F)$ method on samples of photon+jet and dijet events.

The method starts with the transverse momentum balance of the "tag" object that is either a photon, a $Z^{0}$ boson or a jet, and a "probe" jet. At the particle level, the $\vec{p}_{T}$ of the tag $\vec{p}_{T}^{t a g}$ and the hadronic recoil $\vec{p}_{T}^{\text {recoil }}$ are balanced,

$$
\vec{p}_{T}^{\text {tag }}+\vec{p}_{T}^{\text {recoil }}=0 .
$$

The probe jet is part, but not necessarily all of the hadronic recoil present. The response of the tag object $R_{\text {tag }}$ and the hadronic recoil $R_{\text {recoil }}$ might be different, an obvious case being a tag photon and a probe jet. This results in a transverse momentum imbalance as measured by the calorimeter,

$$
R_{\text {tag }} \vec{p}_{T}^{\text {tag }}+R_{\text {recoil }} \vec{p}_{T}^{\text {recoil }}=-{\overrightarrow{E_{T}}}^{\text {meas }} .
$$

From the above equations it is possible to derive the relative response of the hadronic recoil with respect to the tag,

$$
\frac{R_{\text {recoil }}}{R_{\text {tag }}}=1+\frac{{\overrightarrow{E_{T}}}^{\text {meas }} \cdot \vec{n}_{T}^{\text {tag }}}{\vec{p}_{T}^{\text {tag }}},
$$

where ${\overrightarrow{E_{T}}}^{\text {meas }} \cdot \vec{n}_{T}^{\text {tag }}$ is the projection of ${\overrightarrow{E_{T}}}^{\text {meas }}$ direction onto the direction of the tag object transverse momentum. 
The "tag" photon or jet is required to be in the CC, and the "probe" jet can be anywhere in pseudorapidity. The relative response between the "tag" and "probe" objects is related to the missing transverse momentum projected onto the "tag" object direction. The results are shown in Fig. 4.6 for Monte Carlo and data.

- Absolute Calorimeter Response (R): The measured jet energy can be distorted due to a varying response to different particle types, uninstrumented regions of the detector, dead material and a non-linear response as a function of particle energy (Fig. 4.7). It is measured by applying the MPF method to photon+jet events after offset and relative response corrections and is the single largest correction. In order to measure the energy dependence of the jet response with minimal impact from resolution effects, we use $E^{\prime}=p_{T}^{\gamma} \cosh \eta_{j e t}$ to parametrize the absolute response, $\mathrm{R}\left(E^{\prime}\right)$.

- Showering Corrections (S): The showering correction takes into account the energy deposited outside the calorimeter jet cone from particles outside the particle jet as a result of shower development in the calorimeter, magnetic field bending, etc. It is determined in data and Monte Carlo (Fig. 4.8).

Summarizing, jets are calibrated using the jet energy scale (JES):

$$
E^{\text {corr }}=\frac{E^{\text {reco }}-O}{R \times F_{\eta} \times S}
$$

The JES correction factors are derived separately for Monte Carlo and data since the jet response and shower evolution is not modeled perfectly in the Monte Carlo simulation. 


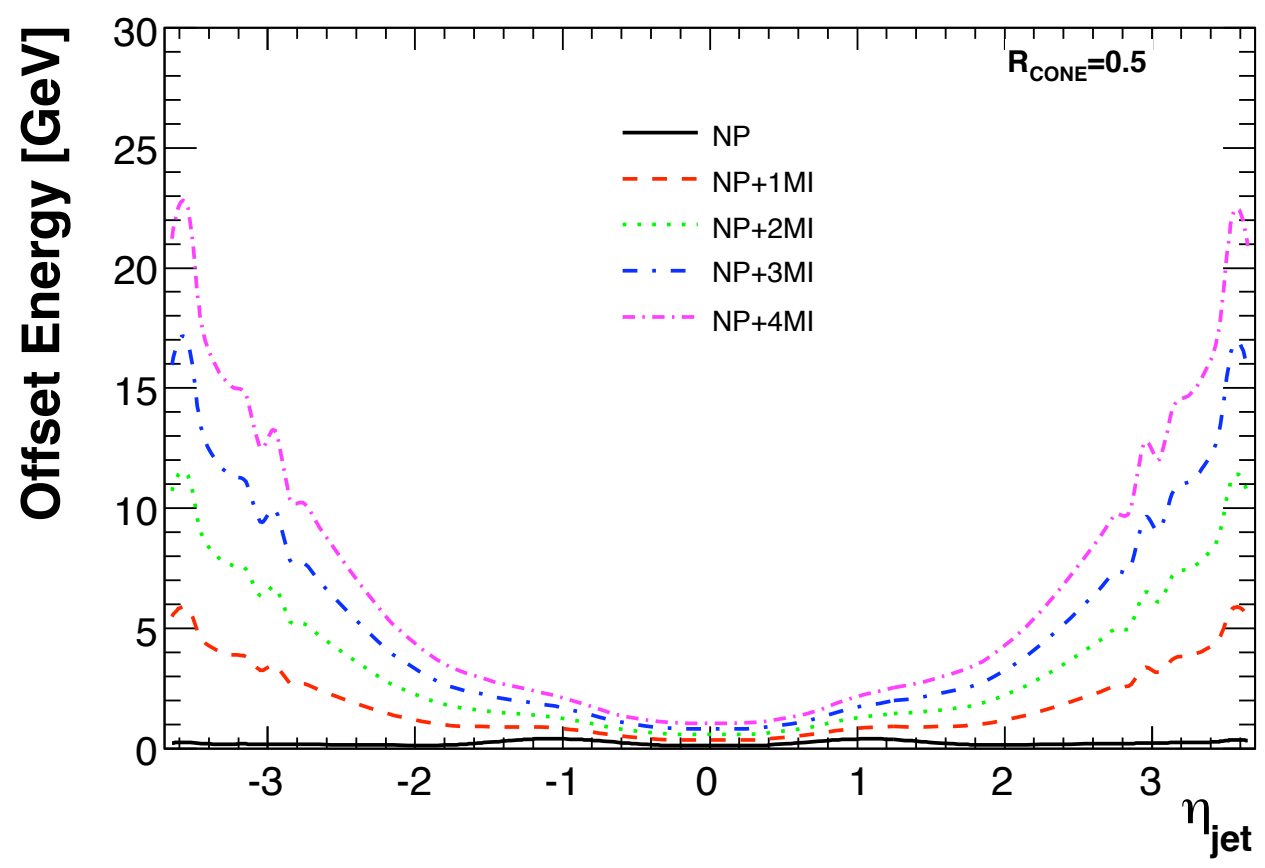

Figure 4.5.: Estimated total jet offset energy (in $\mathrm{GeV}$ ) as a function of $\eta_{\text {jet }}^{\text {det }}$, for jets with $\mathcal{R}_{\text {cone }}=0.5$ [68]. The different lines show the prediction for noise and pile-up $(\mathrm{NP})$ only $\left(n_{P V}=1\right)$, as well as NP and multiple interactions (MI; $n_{P V}>1$ )
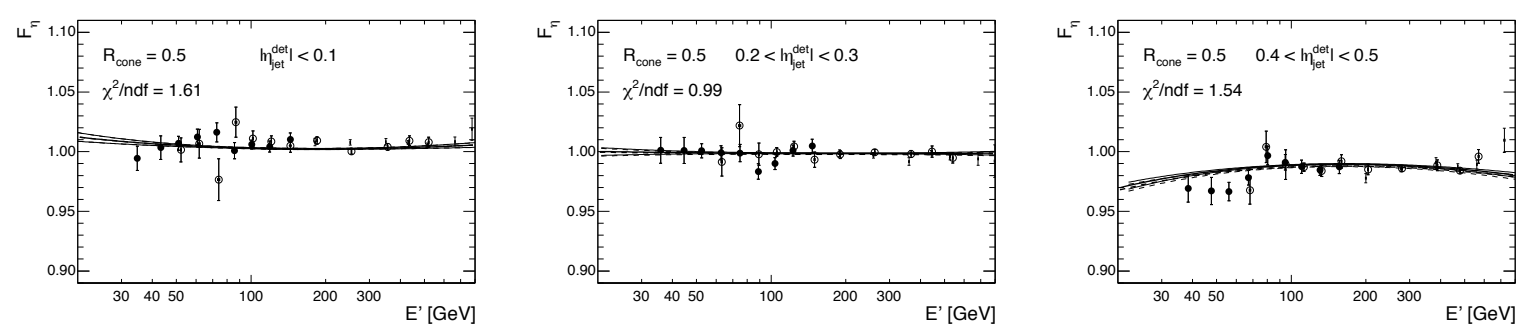

(a) Relative response correction for $\mathcal{R}=0.5$ jets in MC 68.
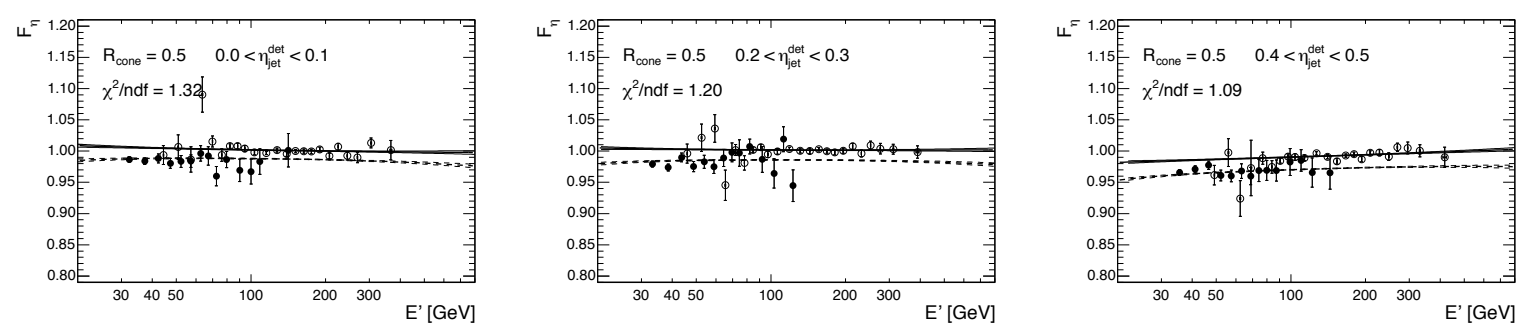

(b) Relative response correction for $\mathcal{R}=0.5$ jets in Data 68.

Figure 4.6.: Examples of relative response correction derived with MPF method for jets in $\mathrm{MC}$ (top) and data (bottom) [68]. Shown are the corrections in $\eta_{\text {det }}<0.1$ (left), $0.2<\eta_{\text {det }}<0.3$ (middle) and $0.2<\eta_{\text {det }}<0.3$ (right) bins. 

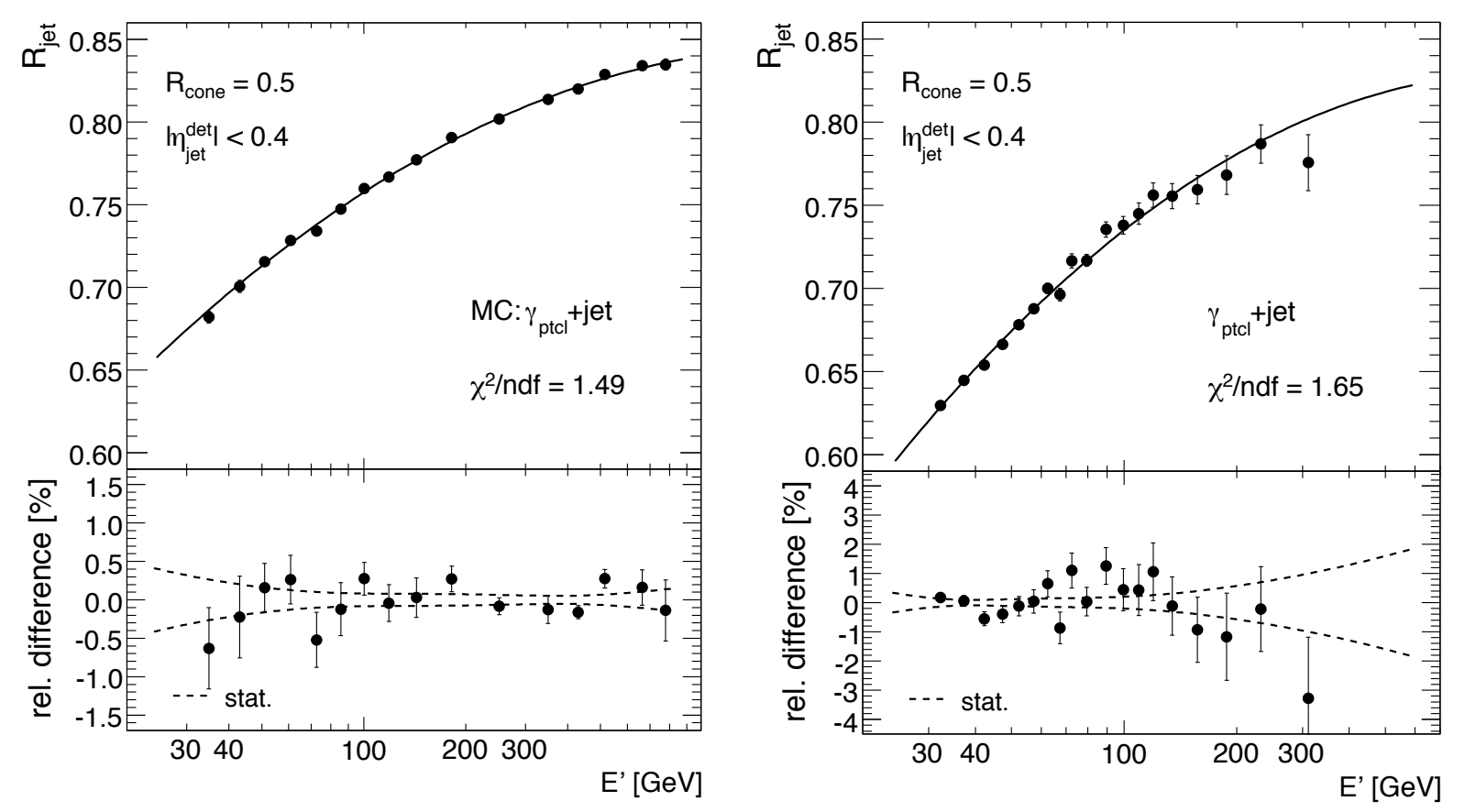

(a) Absolute MPF response for $\mathcal{R}=0.5$ jets in MC (b) Absolute MPF response for $\mathcal{R}=0.5$ jets in data 68. 68.

Figure 4.7.: Absolute response correction derived with the MPF method for jets in MC (left) and data (right) [68].
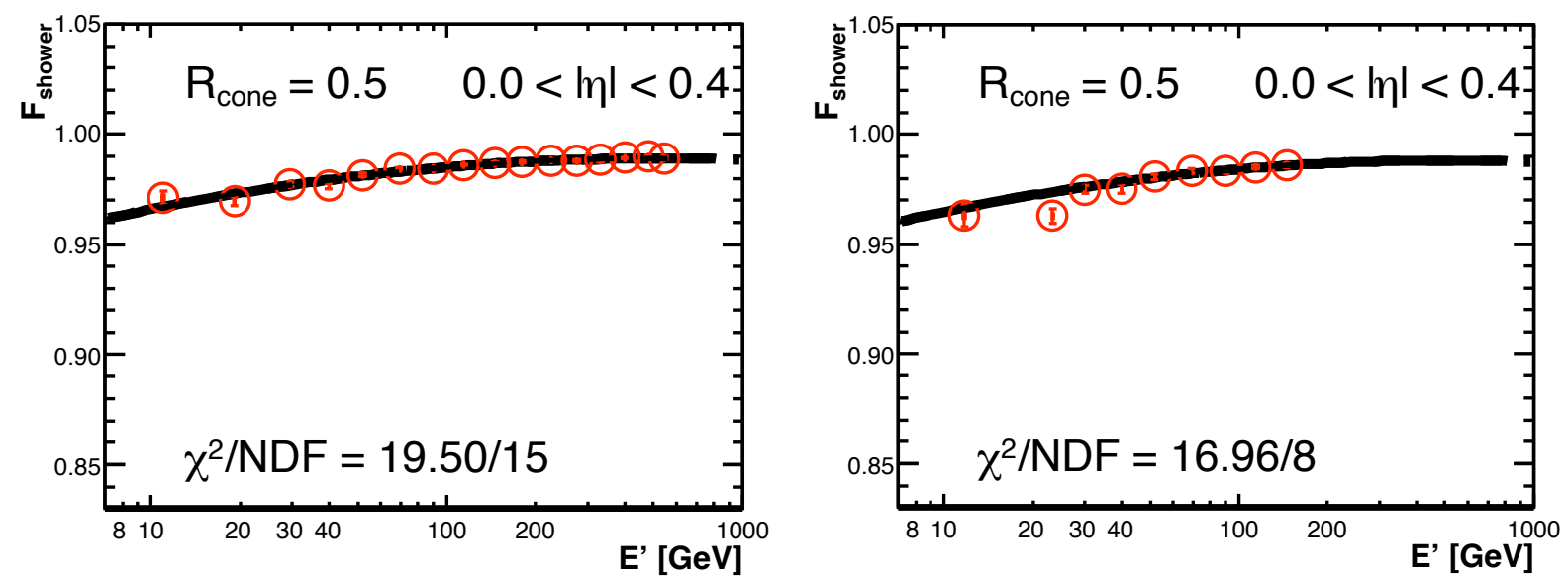

(a) Showering correction for $\mathcal{R}=0.5$ jets in MC 68. (b) Showering correction for $\mathcal{R}=0.5$ jets in Data 68.

Figure 4.8.: Examples of the showering correction in MC (left) and data (right) 68]. 

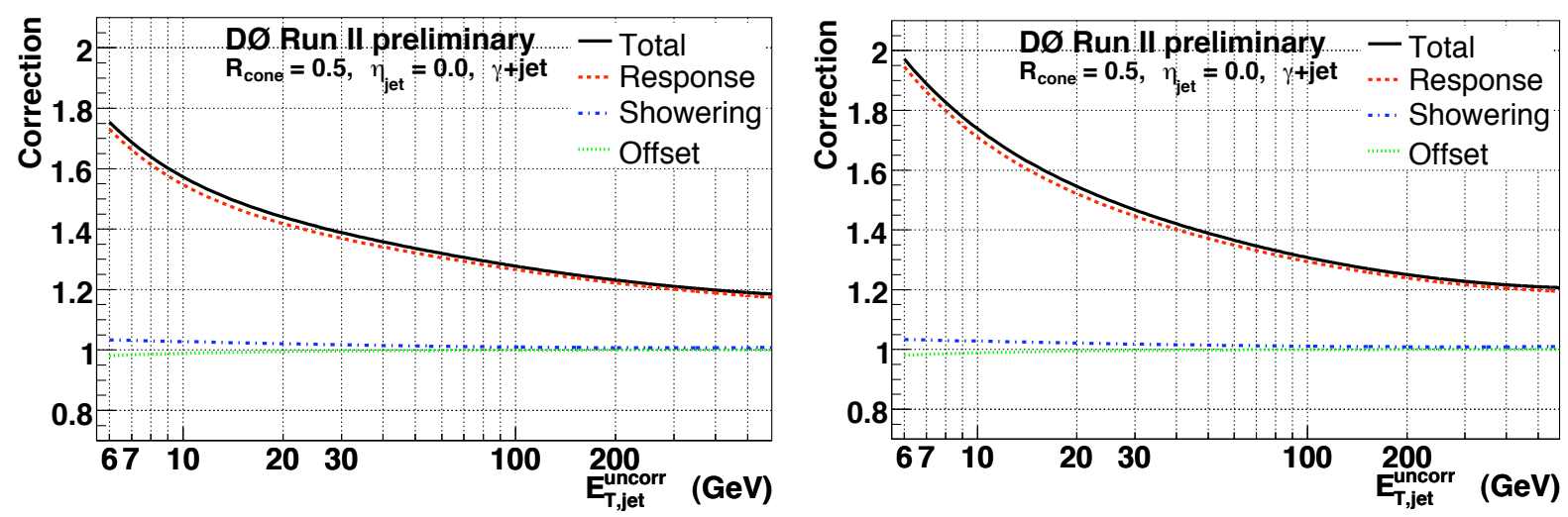

(a) Summary of all JES corrections for $\mathcal{R}=0.5$ jets (b) Summary of all JES corrections for $\mathcal{R}=0.5$ jets with $\eta_{j e t}=0$ in MC [68. with $\eta_{j e t}=0$ in Data [68].

Figure 4.9.: Summary of all JES corrections as a function of uncorrected jet $p_{T}$ in MC (left) and data (right) [68].
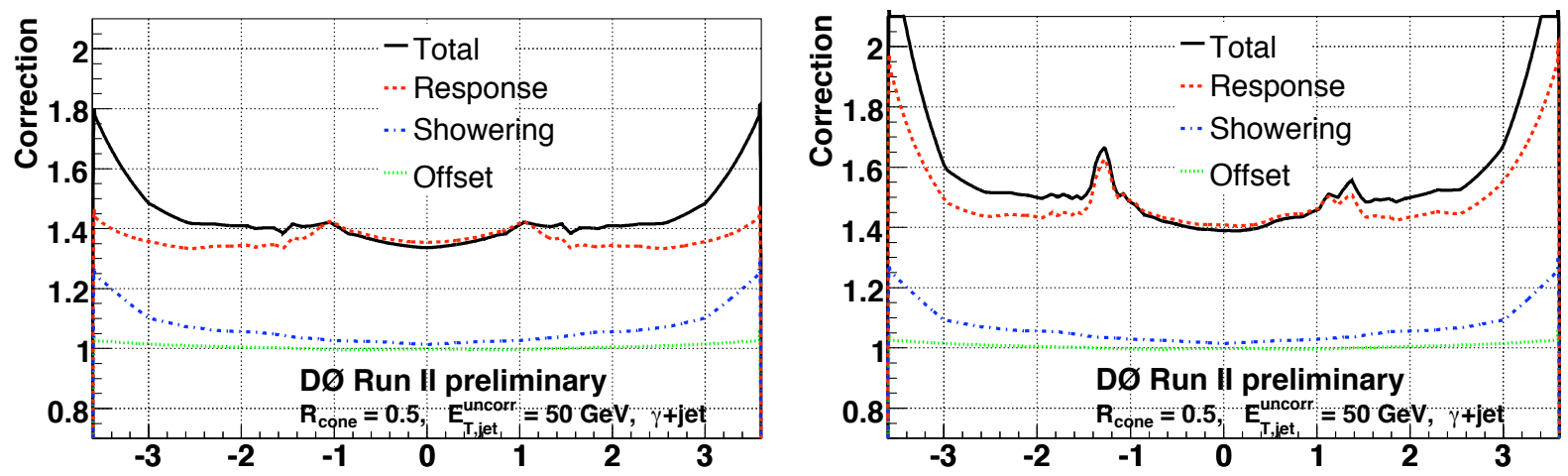

(a) Summary of all JES corrections for $\mathcal{R}=0.5$ jets (b) Summary of all JES corrections for $\mathcal{R}=0.5$ jets with $p_{T, j e t}^{\text {uncorr }}=50 \mathrm{GeV}$ in $\mathrm{MC}$ [68]. with $p_{T, j e t}^{\text {uncorr }}=50 \mathrm{GeV}$ in Data 68.

Figure 4.10.: Summary of all JES corrections as a function of $\eta$ in MC (left) and data (right) for jets with an uncorrected transverse momentum of $50 \mathrm{GeV}$ 68]. 


\subsection{Missing Transverse Energy $\left(\not_{T}\right)$}

The missing transverse energy measures the imbalance of the deposited energy in the calorimeter. This imbalance stems from objects that do not deposit their energy in the detector, e.g. neutrinos. The missing transverse energy corresponds to the negative sum of the calibrated transverse energy in the detector and is calculated from the raw missing transverse energy and additional corrections for the calorimeter response to different physics objects.

The raw missing transverse energy $(M E T B)$ is calculated using all calorimeter cells within the electromagnetic and fine hadronic layers of the calorimeter that have non-zero energy. Cells in the coarse hadronic layer that do not belong to a reconstructed jet with $p_{T}>12 \mathrm{GeV}$ are excluded from the METB calculation.

The raw missing transverse energy has to be corrected for the calorimeter response to electrons and photons, jets, muon and taus. For electron and photon candidates, the difference of the calibrated and uncalibrated energy of physics objects is propagated into $E_{T}$. For jet candidates, the jet energy scale calibration is propagated into the $\mathbb{E}_{T}$ calculation. Muons are minimum ionizing particles that deposit only a small fraction of their total energy in the calorimeter. Their energy deposition in the calorimeter is subtracted from the missing transverse momentum, and the full muon momentum as measured in the muon system and tracker is taken into account.

Due to these corrections, the resolution of the missing transverse momentum is dependent on the physics object resolutions. Moreover, the finite resolution of the calorimeter system and detector effects contribute to the $\mathbb{E}_{T}$ resolution. 


\section{Data Sample and Monte Carlo Simulation}

The first part of this chapter describes the data sample that is used for the analysis presented in this thesis, the trigger selection and data quality requirements. The data quality requirements are necessary to ensure all detector components needed for the reconstruction of the final state physics objects have been in operation.

The second part describes the Standard Model Monte Carlo samples that are used. Due to an incomplete implementation of the detector in the full detector simulation and a missing trigger simulation, efficiency corrections are needed for comparing data and Monte Carlo samples.Moreover, shortcomings of the event generation have to be corrected for, e.g. the instantaneous luminosity distribution of the underlying event in the Monte Carlo samples does not match the luminosity distribution as measured in data.

\subsection{Data Sample}

The data sample used in this thesis has been collected from April 2002 to February 2006. These data can be divided into three subsamples. The first one consists of data reconstructed with software versions $p 17.03 .03$ and $p 17.05 .01$, then fixed with version $p 17.07 .01$ and filtered ("skimmed") with the Common Samples Group (CSG) 2MUhighpt selection for at least two "loose" quality muons with a central track match. In addition, the transverse momentum of the matched central track has to be greater than $10 \mathrm{GeV}$. Due to corrections to the reconstruction (reco) software, the selected data were refixed with reconstruction version p17.09.03. This is the so-called "refixed" sample which corresponds to the SAM dataset definition $C S G_{-} C A F_{-} 2 M U h i g h p t_{-} P A S S 3-p 17.09 .03$.

The next subsample corresponds to data reconstructed with reco version $p 1 \% .09 .00$, rereconstructed with reco version p17.09.06 and skimmed with the 2MUhighpt selection. This sample will be cited as p17.09.06 sample and is contained in the dataset definition CSG_CAF_2MUhighpt_PASS3-p17.09.06b. This dataset also holds part of the data recorded in 2002 that was not reconstructed previously, but added using reco version p17.09.06.

Data originally reconstructed with reco version $p 17.09 .00$, but having a cable swap problem in some calorimeter channels, is the last of the three subsamples. Again, this data was re-reconstructed with $p 17.09 .06$ and skimmed with the $2 M U h i g h p t$ selection. This sample corresponds to the dataset definition $C S G_{-} C A F_{-} 2 M U h i g h p t \_P A S S 3-p 17.09 .06$.

\subsubsection{Data Quality Criteria}

To ensure that all detector components that are necessary to measure physics objects precisely have been in good operation, data quality requirements are imposed. These data 
quality requirements are either evaluated for a whole run, normally corresponding to several hours of data taking, or for a luminosity block, corresponding to one minute of data taking. The data quality criteria removes bad runs due to malfunctioning tracker, muon or online calorimeter as well as bad luminosity blocks due to calorimeter quality being estimated offline to be bad. The data quality also removes events with noise in the calorimeter. Bad luminosity blocks identified by the luminosity tools are removed, too.

\subsubsection{Trigger Selection and Integrated Luminosity}

The rate of $p \bar{p}$ collisions at the Tevatron is significantly higher than the rate at which events can be read out and stored for offline reconstruction. In order to select only interesting events and filter them out, various dedicated triggers that are combined to the triggerlist have been developed (see Sec. 3.2.7). As luminosity increased during Run IIa, the triggerlist was updated and refined. As a result, different trigger list versions were in operation . The analysis presented uses all triggerlist versions available for Run IIa (v8.00 through v14.99).

The choice of muon triggers selected from the triggerlists was motivated by the following requirements. The triggers must be efficient for the event topology under consideration. Moreover, they should not be prescaled for high luminosity running. Prescaling occurs when the rate of a trigger exceeds the dedicated limits. Only a certain fraction of events that fired a prescaled trigger are passed on to the data acquisition system, and the inverse of that fraction is termed prescale. Hence, if a prescale of 1000 is applied to a trigger, every 1000th event that fired this trigger is recorded.

The set of all single muon triggers is the optimal choice for the recent trigger versions. We use the OR of all possible single muon triggers in the triggerlist versions v8.00 through v14.99. The luminosity collected with these triggers in the different triggerlist versions is shown in Table 5.1. Table 5.2 summarizes the single muon triggers that have been considered for the OR.

\begin{tabular}{c||r} 
Triggerlist Version & Integrated Luminosity \\
\hline V8.0 - V10.3 & 24.65 \\
V10.3 - V12.0 & 72.01 \\
V12.0 - V13.0 & 224.08 \\
V13.0 - V13.2 & 33.15 \\
V13.2 - V14.0 & 322.39 \\
V14.0 - V14.6 & 142.06 \\
V14.6 - V15.0 & 190.62 \\
\hline \hline Total & 1008.96
\end{tabular}

Table 5.1.: Integrated luminosity collected with the OR of all single muon triggers and the trigger list version. 


\begin{tabular}{c|c}
\hline Triggerlist Version & Single Muon Triggers \\
\hline \hline V8.0 - V10.3 & MU_W_L2M5_TRK10, MU_W_L2M0_TRK3, \\
& MUW_W_L2M5_TRK10, MU_W_L2M0_TRK10 \\
\hline V10.3 - V12.0 & MU_W_L2M3_TRK10, MUW_W_L2M3_TRK10, \\
& MUW_A_L2M3_TRK10 \\
\hline V12.0 - V13.0 & MU_W_L2M3_TRK10, MUW_W_L2M3_TRK10 \\
\hline V13.0 - V13.2 & MUH1_TK12, MUH1_TK10, MUH1_LM15, \\
& MUH4_LM15, MUH4_TK10, MUH5_LM15, MUH6_TK10, \\
& MUH6_LM15, MUH7_TK10, MUH7_LM15 \\
\hline V13.2 - V14.0 & MUH1_TK12_TLM12, MUH1_TK10, MUH1_LM15, \\
& MUH5_LM15, MUH6_LM15, MUH6_TK12_TLM12, \\
& MUH7_TK12, MUH7_LM15 \\
\hline V14.0 - V14.6 & MUH1_TK12_TLM12, MUH5_LM15, \\
& MUH6_LM15, MUH6_TK12_TLM12, \\
& MUH7_TK12, MUH7_LM15, MUH1_ILM15 \\
\hline V14.6 - V15.0 & MUH1_TK12_TLM12, MUH1_ILM15, \\
& MUH5_LM15, MUH6_LM15, MUH6_TK12_TLM12, \\
& MUH7_TK12, MUH7_LM15, MUH8_TK12_TLM12, MUH8_ILM15 \\
\hline
\end{tabular}

Table 5.2.: List of all single muon triggers available in the single muon trigger OR by triggerlist version. The trigger terms are explained in Tab. 5.3. 


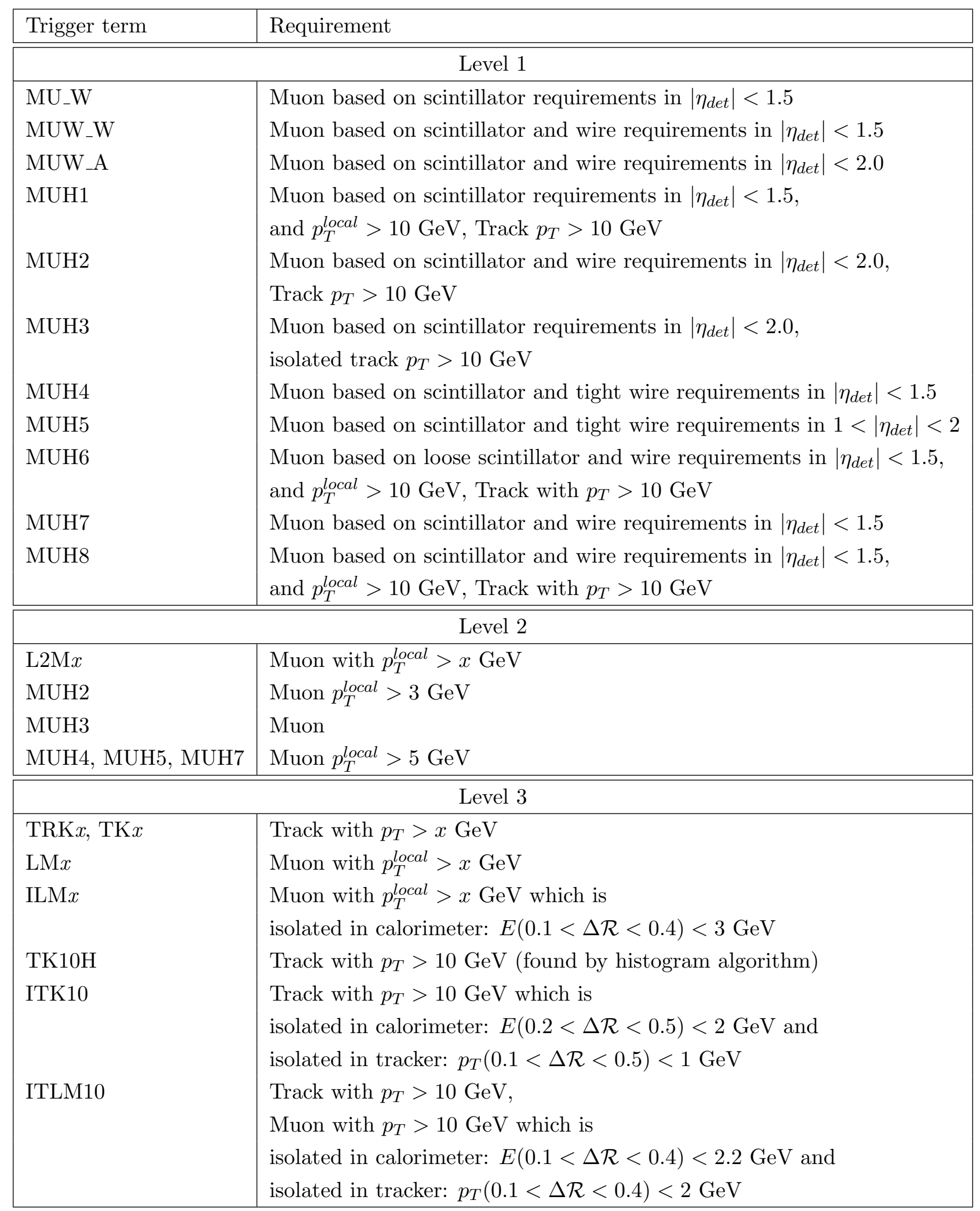

Table 5.3.: Description of the trigger names. $p_{T}^{\text {local }}$ indicates that the muon momentum is measured in the muon detector. The isolation variables are calculated as the scalar sum of the energy or $p_{T}$ in a hollow cone with the given inner and outer radius in calorimeter or tracker, respectively. 


\subsection{Standard Model Monte Carlo Samples}

The signal Monte Carlo samples used in this thesis are $t \bar{t} \rightarrow W^{+} W^{-} b \bar{b} \rightarrow \mu \bar{\mu} \nu \bar{\nu} b \bar{b}$ with $m_{\text {top }}$ from $160 \mathrm{GeV}$ to $185 \mathrm{GeV}$ in $5 \mathrm{GeV}$ steps. Apart from instrumental backgrounds from fake muons, the backgrounds for the dimuon channel are those which manifest the dilepton $+E_{T}$ signature. These processes are diboson production and $Z \rightarrow l \bar{l}$ :

- $p \bar{p} \rightarrow W W+$ jets $\rightarrow \mu \bar{\mu}+j e t s+E_{T}$

Both $W$ s decay to muons and muon neutrinos, giving rise to "real" $E_{T}$. Initial state radiation can lead to additional jets. This is true for all processes described here.

- $p \bar{p} \rightarrow W Z+j e t s \rightarrow \mu \bar{\mu}+j e t s+E_{T}$ and

- $p \bar{p} \rightarrow W Z+j e t s \rightarrow \ell+\nu+\mu \bar{\mu}+j e t s+Z_{T}$

In these processes, the $Z$ decays into two muons, while the jets follow from the hadronic decay of the $W$ and/or initial state radiation depending on the decay mode of the $W$ boson. For hadronically decaying $W$ bosons, mismeasurement can be the source of a significant amount of missing transverse energy.

- $p \bar{p} \rightarrow Z Z+j e t s \rightarrow \mu \bar{\mu}+j e t s+E_{T}$

Again, one of the bosons decays leptonically to two muons. The jets arise either from the hadronic decay of the second $Z$ boson or from initial state radiation. In the latter case, the $2^{\text {nd }} Z$ can decay to two neutrinos, giving rise to "real" $E_{T}$.

- $p \bar{p} \rightarrow Z / \gamma^{*}+j e t s \rightarrow \mu \bar{\mu}+j e t s+E_{T}$ and

- $p \bar{p} \rightarrow Z / \gamma^{*}+j e t s \rightarrow \tau \bar{\tau}+j e t s \rightarrow \mu \bar{\mu}+j e t s+E_{T}$

Just as the signal $t \bar{t}$ decay, the $Z / \gamma^{*} \rightarrow l l$ process creates two opposite sign leptons in the final state. As mentioned before, additional jets can arise from initial state radiation. In the case of $Z / \gamma^{*} \rightarrow \mu \mu$, significant missing transverse energy can arise from mismeasurement while in $Z / \gamma^{*} \rightarrow \tau \tau$ the subsequent $\tau$ decays to neutrinos, leading to "real" missing transverse energy.

\subsubsection{Higher Order QCD corrections}

Higher order contributions to the leading order cross-section consist of virtual corrections and emission of real particles. These QCD corrections can lead to considerable corrections to the leading order cross-section. For hadron colliders, most cross-sections of interest are available at next-to-leading order (NLO), the $Z$ and $W$ production cross -section being available up to next-to-next-to-leading order (NNLO). At NLO, corrections up to $\alpha_{s}$ beyond the leading order Born term are taken into account, while at NNLO corrections up to $\alpha_{s}^{2}$ beyond the leading order cross section are considered.

Higher order corrections not only affect the total cross section, but also differential distributions. However, in most cases it is sufficient to simulate the event topology at LO and use approximations for higher order effects. The total cross-section is corrected using the so-called $K$-factor, which is defined as the ratio of the higher order and leading order cross-section.

$$
K_{(N) N L O} \equiv \frac{\sigma_{(N) N L O}}{\sigma_{L O}}
$$




\subsubsection{Event Generation}

The signal Monte Carlo samples are generated with ALPGEN [69] [70] [71] using the leading order PDF set CTEQ6L1. The top mass has been varied as described above.

The $W W, W Z$ and $Z Z$ backgrounds are studied using samples generated by the package PYTHIA (version 6.319) [72] [73] with the leading order (LO) parton density function CTEQ6L1 from the package LHAPDF (version 3) 74]. In the analysis presented in this thesis, the number of generated diboson events is normalized to the number of events expected from the integrated luminosity of the full Run IIa data sample, $\mathcal{L} \times \sigma_{N L O}$. The next-to-leading order cross section $\sigma_{N L O}$ was calculated with the package MCFM (version 4.1) [75] using parton density function CTEQ6L1 for LO calculation and CTEQ6M for NLO calculation and using the W boson mass as the QCD scale [76].

The $Z \rightarrow \mu \bar{\mu}$ and $Z \rightarrow \tau \bar{\tau}$ backgrounds are studied using samples generated by ALPGEN [69] [70] [71] using leading order (LO) parton density function CTEQ6L1. The computation of the next-to-next-to-leading order (NNLO) cross-section has been performed in [76]. The $Z \rightarrow \mu \bar{\mu}$ and $Z \rightarrow \tau \bar{\tau}$ ALPGEN sample is scaled to the NNLO expectation using the integrated luminosity of our data sample, $1008.96 \mathrm{pb}^{-1}$. The used Monte Carlo samples, the LO and (N)NLO cross sections are listed in Table 6.1.

\section{Combining ALPGEN and PYTHIA Monte Carlo}

ALPGEN is a matrix element (ME) only event generator, i.e. the generated partons neither undergo fragmentation nor hadronization. This is still done using PYTHIA. However, a given $Z+\mathrm{n}$ jet event can be generated by $\mathrm{n}$ partons through soft/collinear radiation or by (n1) partons through soft/collinear radiation and additional hard, large angle gluon emission [77. Thus, a mechanism to avoid double counting and dead regions of the available phase space has to be applied. The algorithm implemented by ALPGEN consists of three steps. First, partons from the ME calculation are showered without any veto on hard emissions during the shower. After this evolution, a jet cone algorithm with cone size $R_{\min }=0.7$ and minimum transverse energy $E_{T}=8 \mathrm{GeV}$ is applied to the final parton state and partons from the ME calculation are matched to jets. A parton is considered to be matched if the distance between the parton and the jet centroid is smaller than $R_{\min }$.

The partons from the ALPGEN matrix element must match the jets from the PYTHIA parton shower. For exclusive samples it is furthermore required that no extra jet is present. Only in the case of highest partonic multiplicity, additional jets are allowed. This sample is called inclusive. When estimating the background due to a process that is modeled by ALPGEN Monte Carlo and subsequent PYTHIA fragmentation and hadronization, the samples with the different light parton content have to be normalized to the same luminosity according to their ALPGEN cross-sections before combining them. With this procedure, one recovers e.g. the $Z+$ light jets sample from the exclusive $Z+0$ light parton $(l p)$ up to and including the $Z+(\mathrm{n}-1) l p$ samples and the $Z+$ nlp inclusive sample, where $\mathrm{n}$ is the maximum number of hard parton emissions included in the matrix element.

\subsubsection{Modeling of Initial and Final State Radiation}

Initial and/or final state radiation (ISR and FSR, respectively) may appear in every process that contains colored and/or charged particles and give rise to corrections of the event topology and cross section. PYTHIA takes this into account by using the parton shower 
method. This method approximates the effects of ISR and/or FSR in a probabilistic method by an evolution of a series of branchings of a mother parton into two daughter partons. The momentum fractions are chosen to be $z$ and $1-z$, and the branching is described by the DGLAP [78] splitting functions. Starting from the hard interaction, the splitting is either performed forward (for FSR) or backward (for ISR) and cut off at some scale $Q_{0}$ that is smaller than the hard scatter scale $Q_{\max }^{2}$. The parton shower method gives good description of collinear and soft radiation, but has limited predictive power for the emission of hard and wide-angle partons.

\subsubsection{Fragmentation and Hadronization}

Perturbative QCD is only valid at short distances, while it breaks down at large distances due to the evolution of the coupling constant for larger scales (and therefore smaller momentum transfers $Q$ ). In the confinement regime, colored partons are transformed into colorless hadrons. This process is called fragmentation and hadronization.

The fragmentation of partons into hadrons cannot be calculated and is described in analogy to the partonic structure of the proton: fragmentation functions give the probability that a quark produces a hadron with a given fraction of the quark's energy. The most successful theoretical model describing fragmentation is the string fragmentation model. In this model, quarks are bound by a string with a certain energy density between the partons that move apart. Quark-antiquark pairs are created along the string in a way that the string breaks up into stable and unstable hadrons. The decay of unstable hadrons is simulated using decay matrix elements or results of measurements.

The fragmentation functions have been measured at LEP [18].

\subsubsection{Detector Simulation and Event Reconstruction}

The particles produced by the Monte Carlo generator are passed through the detector simulation, which is handled by the programs DØGSTAR and DØSIM.

DØGSTAR simulates the interaction of particles with the detector. It is based on GEANT [79], a software program that simulates the passage of elementary particles through matter. All subdetectors are modeled in detail and the hits and energy depositions in the active detector parts are determined. This step is the most time consuming in the whole event simulation process, since the software has to keep track of all particle interactions in detail.

In the next step, the simulated events are modified to account for effects due to the difference between the real and the simulated detector. Zero bias events and pile-up from previous bunch crossings are added to the event by DØSIM, which also includes the simulation of noise from the detector and electronics. Its output is the same format as the data recorded by the data acquisition system. It also contains additional Monte Carlo information which makes it possible to correlate the detector data with the event generator output.

The output of the detector simulation is then reconstructed with the same reconstruction software as data, DØRECO. In the present thesis software version p17 is used for all three programs. 


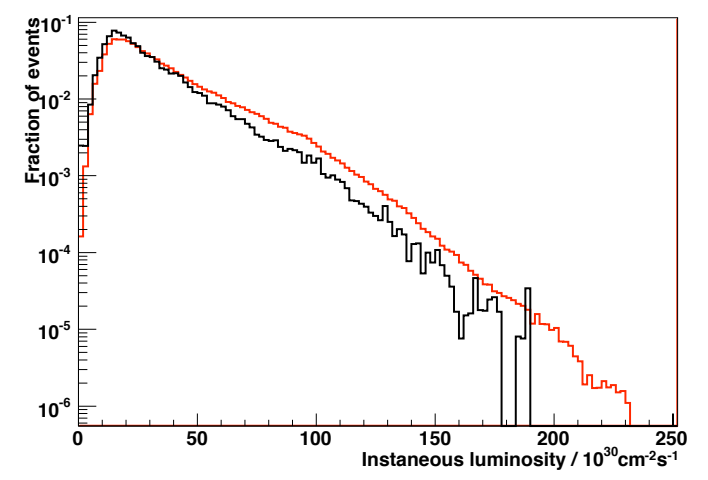

(a) Data (red) and $t \bar{t}$ MC (black) luminosity distribution before reweighting.

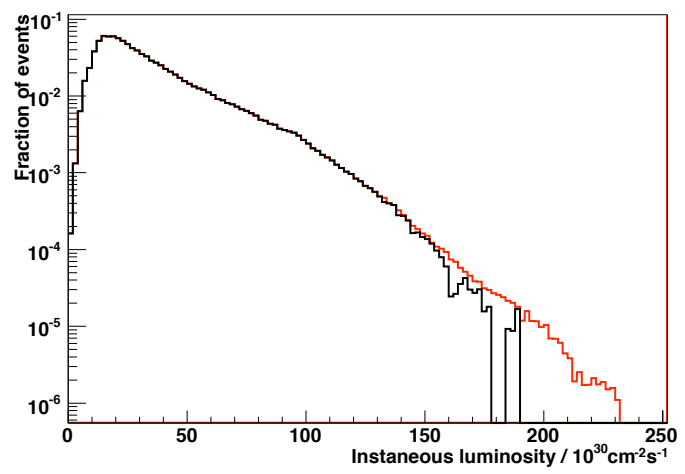

(b) Data (red) and $t \bar{t}$ MC (black) luminosity distribution after reweighting.

Figure 5.1.: Comparison of the instantaneous luminosity distribution in Run IIa data and the luminosity distribution of the overlaid zero bias event in $t \bar{t}$ Monte Carlo before and after reweighting. The distributions were normalized to unit area.

\subsubsection{Monte Carlo Corrections}

The various signal and background Monte Carlo samples are used to estimate the number of Standard Model background events at each selection stage and to determine selection efficiencies. Moreover, they are used to optimize the event selection in order to suppress the Standard Model background while retaining a high signal selection efficiency. It is therefor required that the simulated events reproduce the data and apply corrections to the simulated events where necessary.

The Monte Carlo samples do not reproduce the data correctly for two reasons. First, the event generation is based on leading order matrix element matched with parton shower Monte Carlo and therefore does not include e.g. higher order virtual corrections. Moreover, the zero bias events added by DØSIM do not reproduce certain distributions as found in data.

Second, triggers are not simulated in Monte Carlo and the implementation of the DØ detector in GEANT is incomplete. These effects lead to corrections of the object identification efficiency and energy and momentum resolutions that have to be determined in data or by comparing data with Monte Carlo.

\section{Luminosity Distribution and Vertex z Position Corrections}

The zero bias events picked randomly by DØSIM and overlaid on the generated events reproduce a certain luminosity distribution that is different than in data. Moreover, the event simulation uses parameters for the beam overlap region that are different to the ones found in data. These differences between data and Monte Carlo are addressed by two corrections applied to the Monte Carlo samples.

First, to correct the luminosity distribution, the instantaneous luminosity distribution of the data sample and the different Monte Carlo samples is determined. In a second step, these distributions are used to calculate event weights and the Monte Carlo events are reweighed accordingly. Fig. 5.1 shows the effect of the reweighting on the instantaneous 
luminosity distribution of the overlaid zero bias event in $t \bar{t}$ Monte Carlo.

Second, to reproduce the longitudinal shape of the luminous beam region and the primary vertex $\mathrm{z}$ of the data sample in our Monte Carlo samples, the fraction of integrated luminosity in each run number range with constant beam parameters is determined. Next, the primary vertex $\mathrm{z}$ distribution is measured in different instantaneous luminosity bins and the same run ranges used before that have constant beam optics. The shape of the luminous region as given by 80 ]

$$
\frac{d \mathcal{L}}{d z}=N_{p} N_{\bar{p}} \frac{1}{\sqrt{2 \pi} \sigma_{z}} \frac{1}{4 \pi \sigma_{x}(z) \sigma_{y}(z)} e^{-\frac{\left(z-z_{0}\right)^{2}}{2 \sigma_{z}^{2}}}
$$

is fit to the vertex $\mathrm{z}$ distribution to find the length and position of the proton-antiproton beam overlap region and its center $\mathrm{z}$ coordinate, $\sigma_{z}$ and $z_{0}$, and the overall normalization $N=N_{p} N_{\bar{p}} \frac{1}{\sqrt{2 \pi} \sigma_{z}} \frac{1}{4 \pi \sigma_{x}(z) \sigma_{y}(z)}$. The transverse widths $\sigma_{x}(z)$ and $\sigma_{y}(z)$ of the beam spot vary as a function of $z$. Their measurement is described in [81]. The vertex $z$ distribution in the simulation is Gaussian with the center at the detector origin and a width of $\sigma_{z}^{M C}=25$ $\mathrm{cm}$. Thus, the event weight given by

$$
w=\frac{N_{p} N_{\bar{p}} \frac{1}{\sqrt{2 \pi} \sigma_{z}} \frac{1}{4 \pi \sigma_{x}(z) \sigma_{y}(z)} e^{-\frac{\left(z-z_{0}\right)^{2}}{2 \sigma_{z}^{2}}}}{\frac{1}{\sqrt{2 \pi}\left(\sigma_{z}^{M C}\right)^{2}} e^{\left.-\frac{(z)^{2}}{2 *(\sigma M C}\right)^{2}}}
$$

is applied to all Monte Carlo events based on the run number and instantaneous luminosity of the overlaid zero bias event.

\section{Muon Efficiency and Resolution Corrections}

The muon identification efficiencies are determined with a tag and probe method that is implemented in the software packages wzreco and muo_cert. $Z \rightarrow \mu \bar{\mu}$ events are selected by wzreco using muon quality, muon to central track matching and isolation requirements on the tag muon. The probe muon needs only to pass central track matching and isolation criteria. This probe is then matched to muon objects of different qualities to estimate the reconstruction efficiency [59]. The ratio of the reconstruction efficiency in data and Monte Carlo as a function of $\eta_{\text {det }}$ and $\phi$ serves as an efficiency correction factor. Fig. 5.2 shows the efficiency correction for "loose" quality muons as a function of $\eta_{\text {det }}$.

The track matching and isolation efficiencies are measured using the same technique, but removing the central track matching or isolation requirement on the probe muon, and requiring the probe muon to be of loose quality. The track matching efficiency correction is parametrized in $\eta_{C F T}$ and $z$, while the isolation efficiency is parametrized in the number of jets with $p_{T}>15 \mathrm{GeV}$, njet15. The track matching efficiency correction as a function of $\eta_{\text {det }}$ is depicted in Fig. 5.3 .

Muon efficiency corrections are applied to Monte Carlo events in the form of event weights.

The momentum resolution is estimated from the width of the $Z^{0}$ peak [59]. However, in fitting the $Z^{0}$ peak one has to consider that the peak width and shape is the result of the convolution of the Drell-Yan spectrum and the muon resolution. The dimuon mass resolution is due to the measurement uncertainty on the muon momentum, which varies 


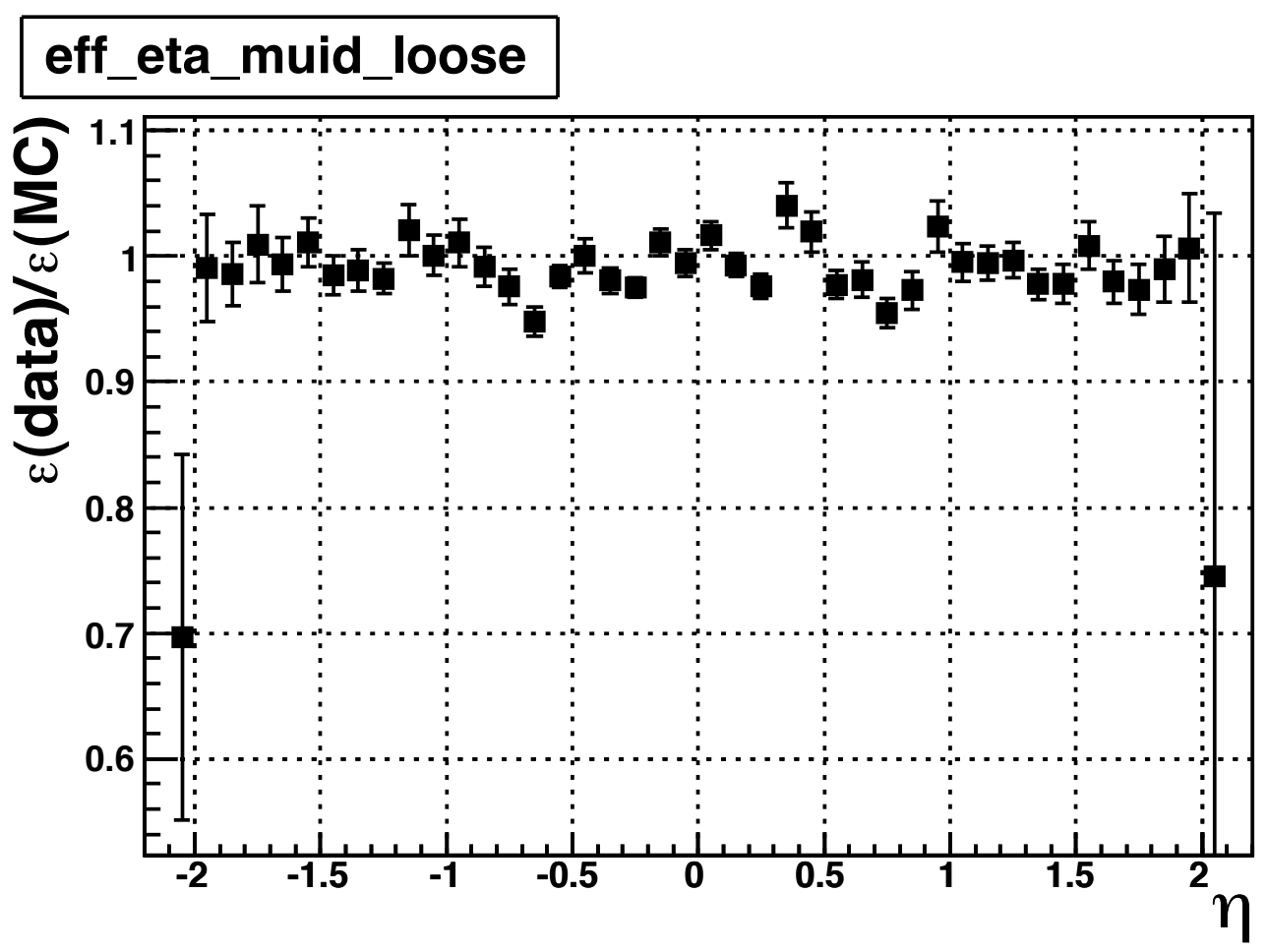

Figure 5.2.: Relative muon identification efficiency correction [59].

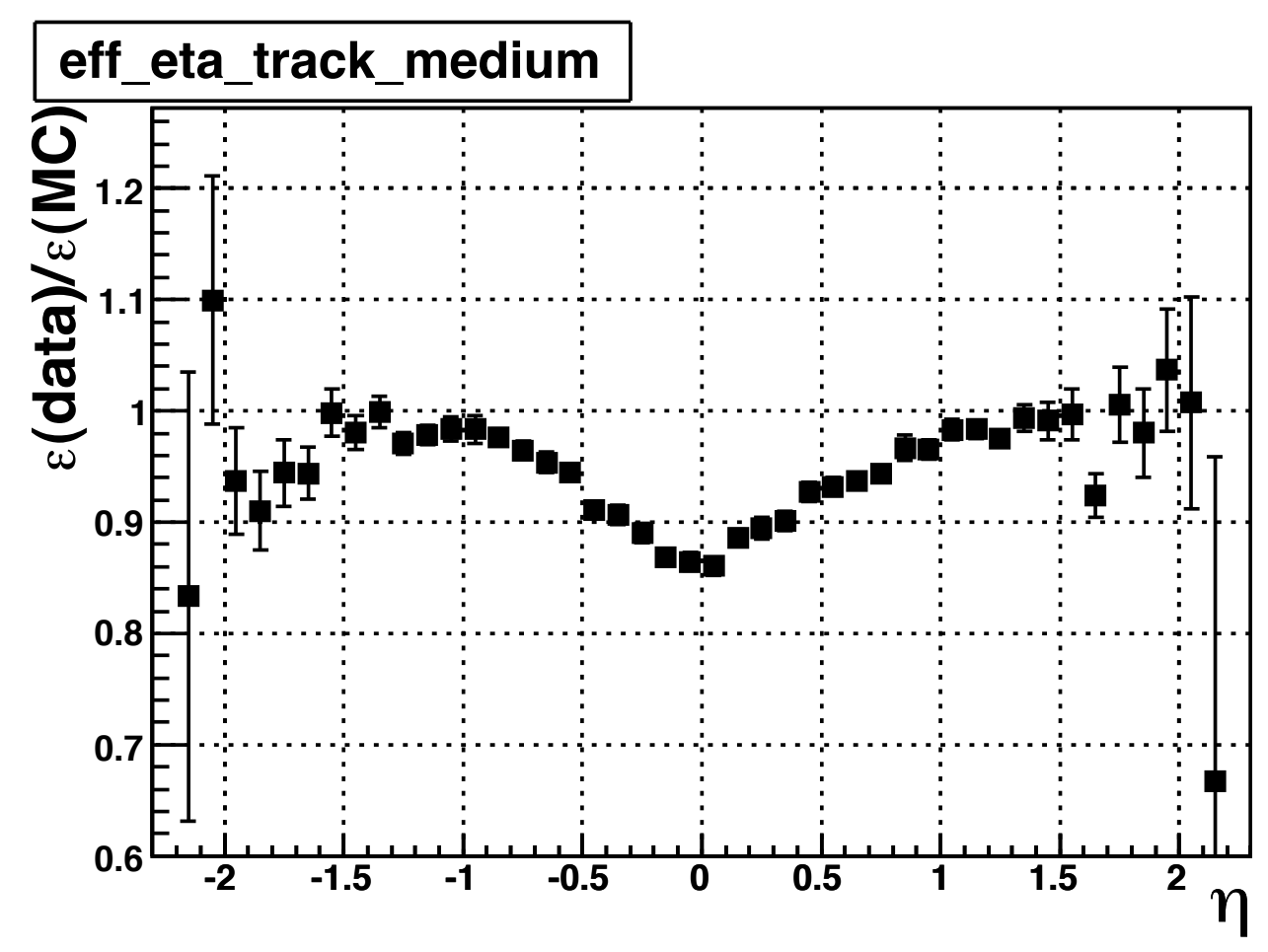

Figure 5.3.: Relative track matching efficiency correction (as found in [59]). 

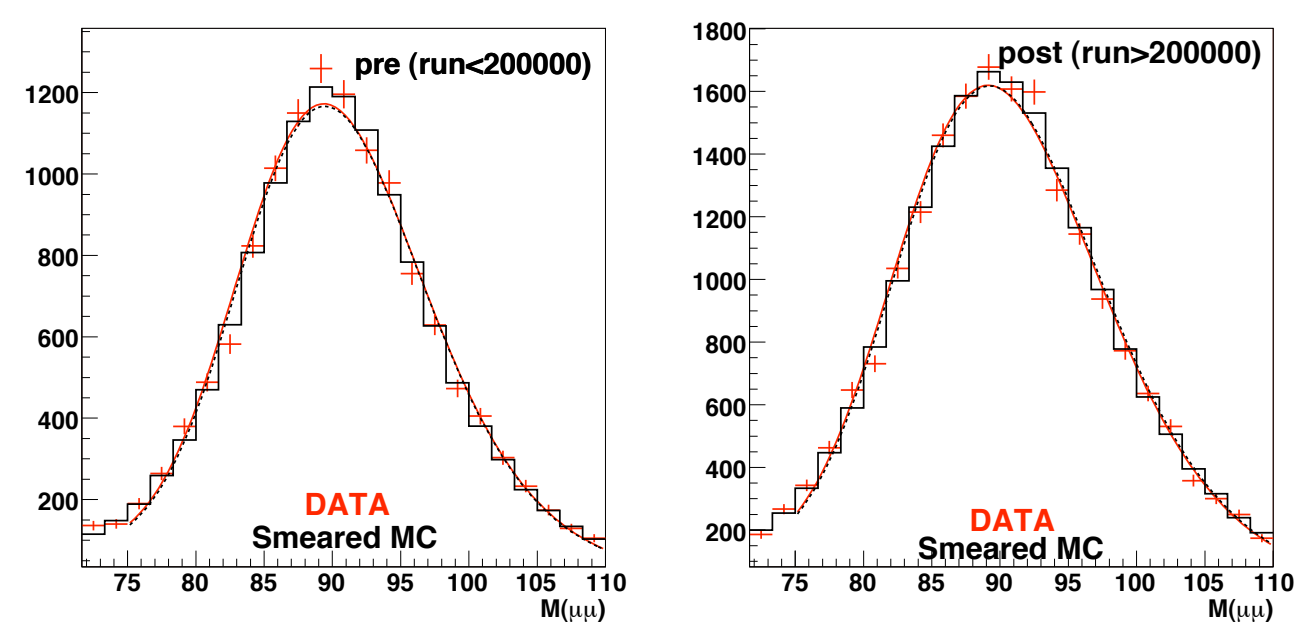

Figure 5.4.: Comparison of the $Z$ peak in $Z \rightarrow \mu \bar{\mu}$ events in data and MC where both muons have SMT hits and $\left|\eta_{C F T}\right|<1.6$ (as found in [59]). In MC, the muon $p_{T}$ was smeared according to eq. 5.4 .

as $1 / p_{T}$. Thus, each muon may have a different resolution. In the Monte Carlo samples, the muon momenta are smeared using [59]

$$
q / p_{T}^{\text {smeared }}=q / p_{T}+\left(A+B / p_{T}\right) \times \text { Random }
$$

where $\mathrm{q}$ is the muon charge, Random is a Gaussian distributed random number (with mean $=0$ and width $=1), p_{T}\left(p_{T}^{\text {smeared }}\right)$ is the (smeared) muon transverse momentum and $\mathrm{A}$, $\mathrm{B}$ are parameters chosen such that the $Z^{0}$ peak has the same width and shape in Monte Carlo and data. Three different parameter pairs of A and B are used: One for muons with a matched central track without hits in the SMT, one for central tracks that have SMT hits and $\left|\eta_{\text {det }}\right| \leq 1.6$ or $\left|\eta_{\text {det }}\right|>1.6$ each. After smearing, the width of the $Z^{0}$ peak in data and the simulated event samples shows good agreement as can bee seen in Fig. 5.4. Fig. 5.5 visualizes the uncertainty introduced by the muon momentum smearing. This is done by varying the smearing parameters by their uncertainty up and down by one $\sigma$.

\section{Muon Trigger Efficiency}

Starting from the efficiencies of the single muon triggers measured with the software packages wzreco and muo_cert, the efficiency of the single muon trigger OR can be calculated for muons with different qualities, track matching and isolation requirements in analogy to the muon reconstruction efficiency as follows (cf. [82]). The probability to trigger for an event with a single muon is $P\left(\eta_{\text {det }}, \phi\right)$. For a dimuon event the probability of the first muon, $P\left(\eta_{d e t}^{1}, \phi^{1}\right)$, has to be added to the probability that the first muon didn't trigger but the second did, $\left(1-P\left(\eta_{\text {det }}^{1}, \phi^{1}\right)\right) \times P\left(\eta_{\text {det }}^{2}, \phi^{2}\right)$, to determine the probability that at least one of the single muon triggers fired. The trigger efficiencies for events with more than two muons can be derived in analogy to the two muon case. The trigger efficiencies were parametrized in detector $\eta$ and the polar angle $\phi$ to account for the muon spectrometer geometry. As an example, Fig. 5.6 shows the trigger efficiency for the triggerlist versions 13.2 through 13.99. The efficiency is then applied to a simulated event with one or more 


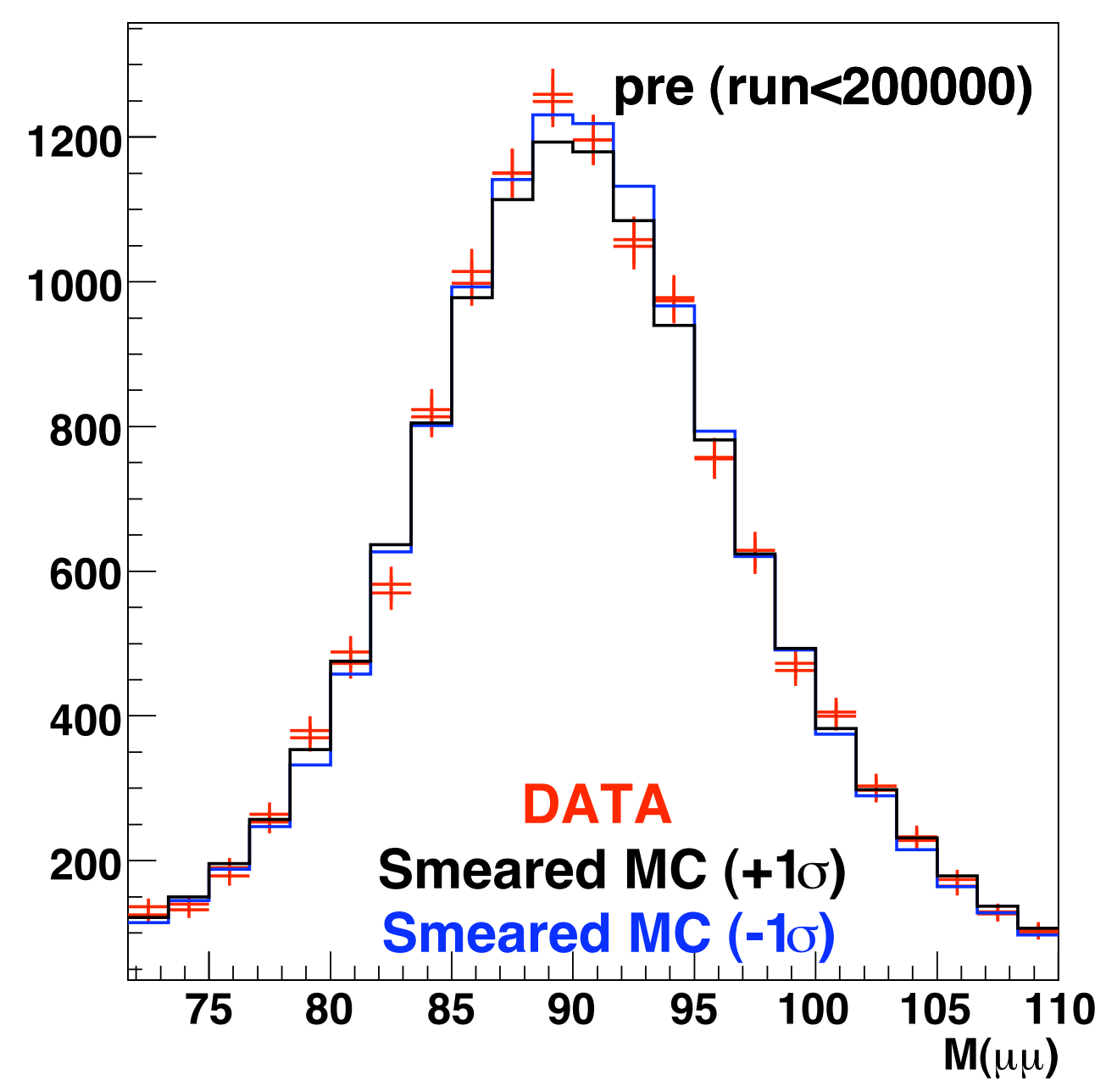

Figure 5.5.: Comparison of data and smeared Monte Carlo for events where both muons have SMT hits and $\left|\eta_{C F T}\right|<1.6$ (as found in [59]). The two Monte Carlo distributions represent the $\pm 1 \sigma$ variation of the average smearing. The two data error bars represent different track qualities (medium and none). 


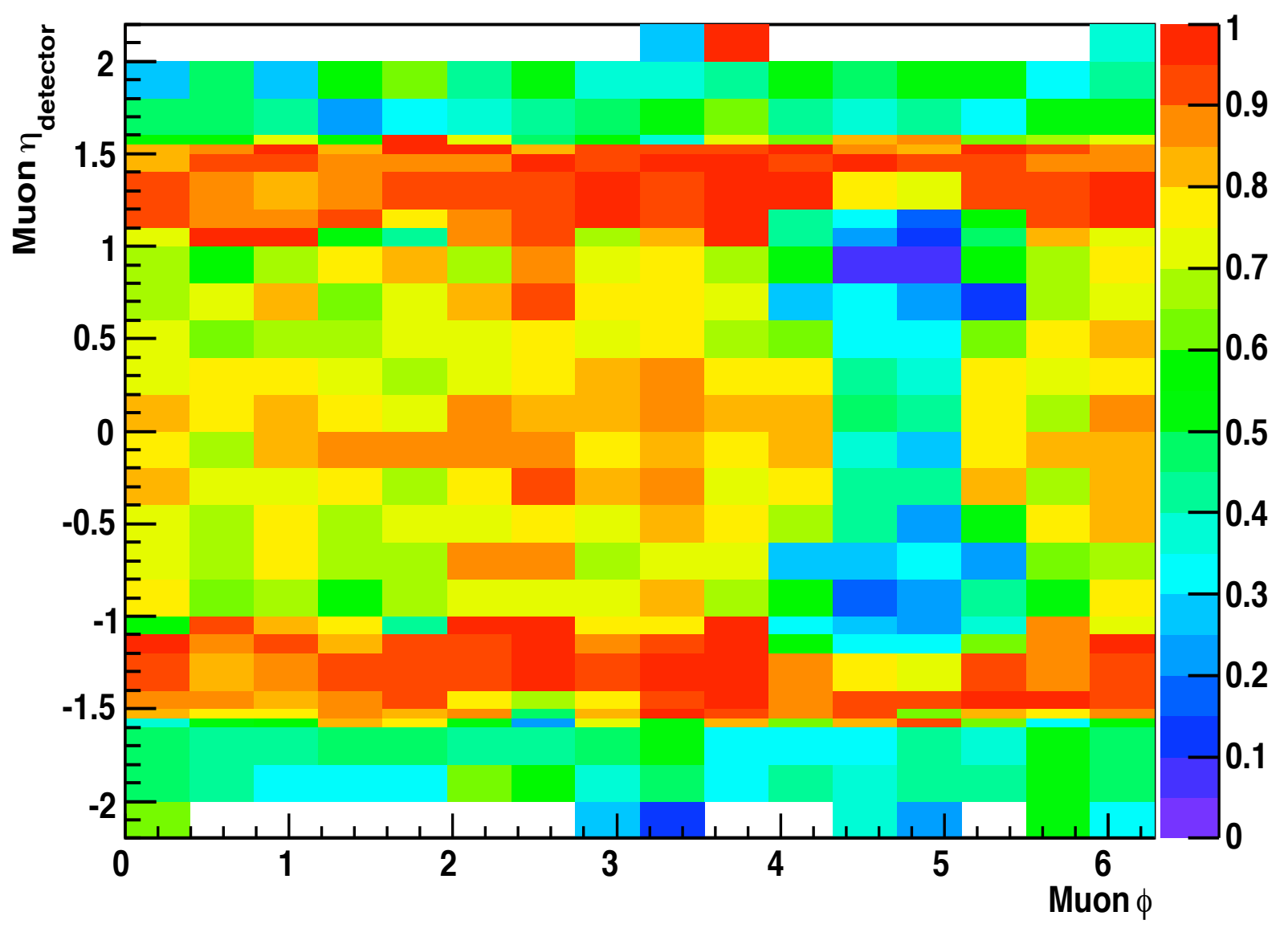

Figure 5.6.: Efficiency of the logic OR of all single muon triggers in the triggerlists v13.2v13.99 (taken from [82]) for loose quality, medium track matched muons.

suitably selected muons as follows:

$$
\begin{aligned}
P & =\sum_{i=1}^{N_{\text {muons }}} P\left(\eta_{\text {det }}^{i}, \phi^{i}\right) \prod_{j=1}^{i-1}\left(1-P\left(\eta_{\text {det }}^{j}, \phi^{j}\right)\right) \\
& =1-\prod_{i=1}^{N_{\text {muons }}}\left(1-P\left(\eta_{\text {det }}^{i}, \phi^{i}\right)\right)
\end{aligned}
$$

where $P\left(\eta_{\text {det }}^{i}, \phi^{i}\right)$ is the efficiency (probability) of the ith muon with the latter coordinates to pass the $\mathrm{D} \varnothing$ trigger requirements (as measured in data), $N_{\text {muons }}$ is the number of muons in the event under consideration and $P$ is the total trigger efficiency (probability) the event weight is multiplied with.

\section{Jet Corrections: Jet Smearing, Shifting and Removal}

Jet smearing, shifting and removal (JSSR, jet $S S R)$ is a procedure to recalibrate, smear and discard jets in a consistent way to reach agreement of the simulated jets and jets in data [83. The main tool to determine these corrections with data is the $p_{T}$ imbalance

$$
\Delta S=\frac{p_{T}^{j e t}-p_{T}^{\gamma}}{p_{T}^{\gamma}}
$$


in photon + jet events.

Its distribution in different ranges of $p_{T}^{\gamma}$ is fitted with Gaussians multiplied by the reconstruction and identification efficiency which are parametrized as error functions to gain information on

- the jet energy scale via the Gaussians' central values,

- the jet energy resolution via the width of the Gaussians,

- the jet reconstruction and identification efficiencies via the turn-on curves and the ratio of the area under the full Gaussian versus the area under the Gaussian multiplied by the error function.

Fig. 5.7 shows $\Delta S$ distributions including the aforementioned fit and its uncertainty.

After $\eta$-dependent corrections, the differences of parameters in Monte Carlo and data are used to oversmear jets in Monte Carlo events. Next, jets are shifted and in a final step removed according to the reconstruction and identification efficiencies. 
$\Delta \mathrm{S}$ distribution $(18<$ photon $\mathrm{pT}<23)$

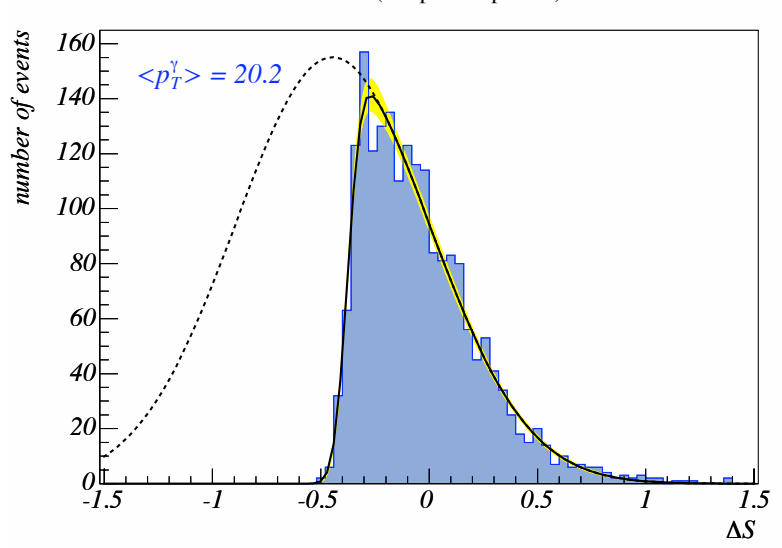

$\Delta \mathrm{S}$ distribution $(70<$ photon $\mathrm{pT}<75)$

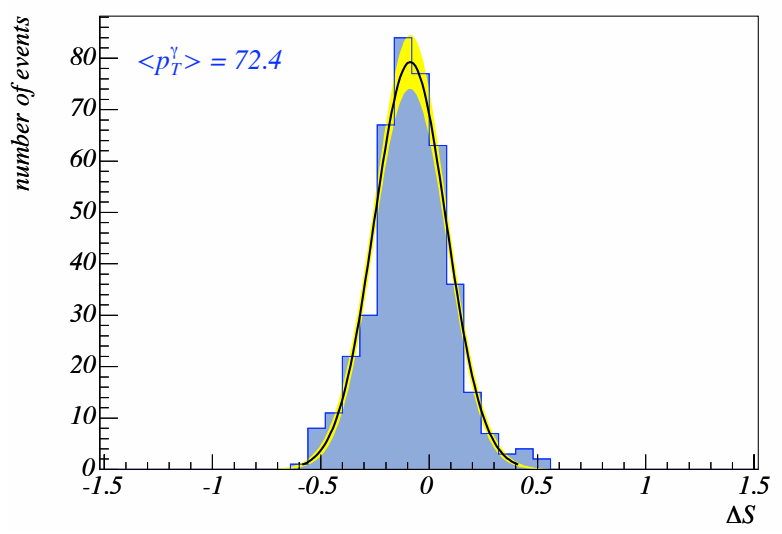

$\Delta \mathrm{S}$ distribution $(18<$ photon $\mathrm{pT}<23)$

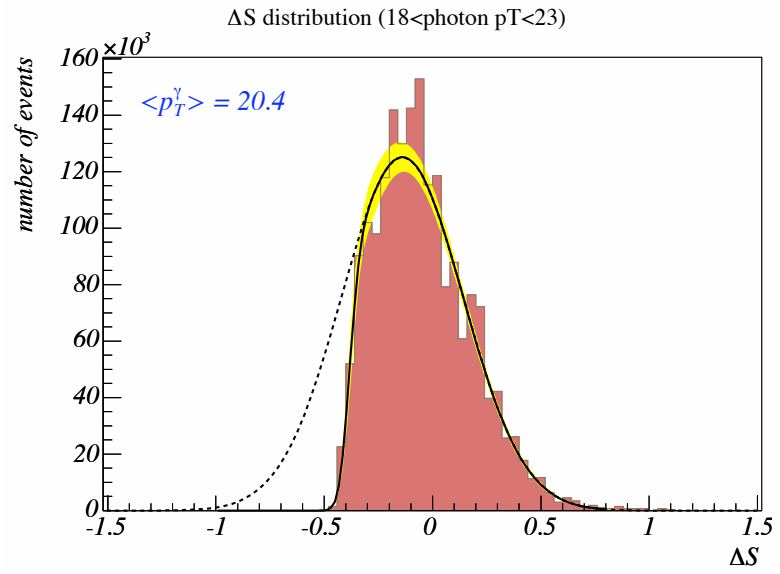

$\Delta \mathrm{S}$ distribution $(70<$ photon $\mathrm{pT}<75)$

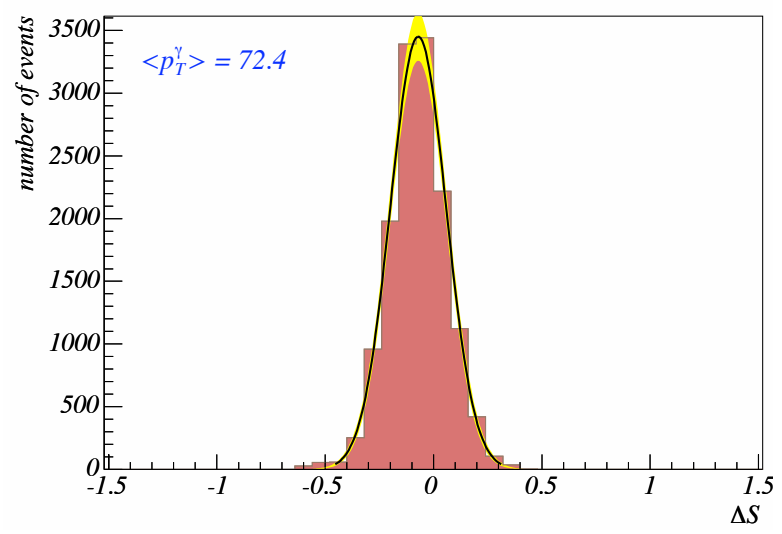

Figure 5.7.: Distributions of $\Delta S$ for different ranges of $p_{T}^{\gamma}$ (taken from [83]). In the upper plots $\left(18<p_{T}^{\gamma}<23\right)$ fits by the product of an error function and a Gaussian are superimposed. The dashed curve corresponds to an extrapolation of the Gaussian part of the fit function in the region affected by the turn-on. In the bottom plots $\left(70<p_{T}^{\gamma}<75\right)$ the $\Delta S$ distribution is fitted by a Gaussian. The yellow bands indicate the statistical error. 


\section{Measurement of the Top Quark Pair Production Cross Section in the Dimuon Final State}

\subsection{Signal Monte Carlo}

The signal process, $p \bar{p} \rightarrow t \bar{t} \rightarrow b \bar{b}+\mu \bar{\mu}+E_{T}$ (see Fig. 6.1) is simulated with ALPGEN [69] [70] [71], and the simulated events are passed through PYTHIA [72] [73] for the parton showering and modeling of ISR, FSR and the underlying event. In addition to signal samples with a generated top mass of $m_{\text {top }}=165 \mathrm{GeV} m_{\text {top }}=175 \mathrm{GeV}$ and $m_{\text {top }}=185 \mathrm{GeV}$, smaller samples with $m_{\text {top }}$ between $160 \mathrm{GeV}$ and $190 \mathrm{GeV}$ in $5 \mathrm{GeV}$ steps were produced with PYTHIA only. The different signal samples allow for parametrization of the signal selection efficiency as a function of top mass later on.

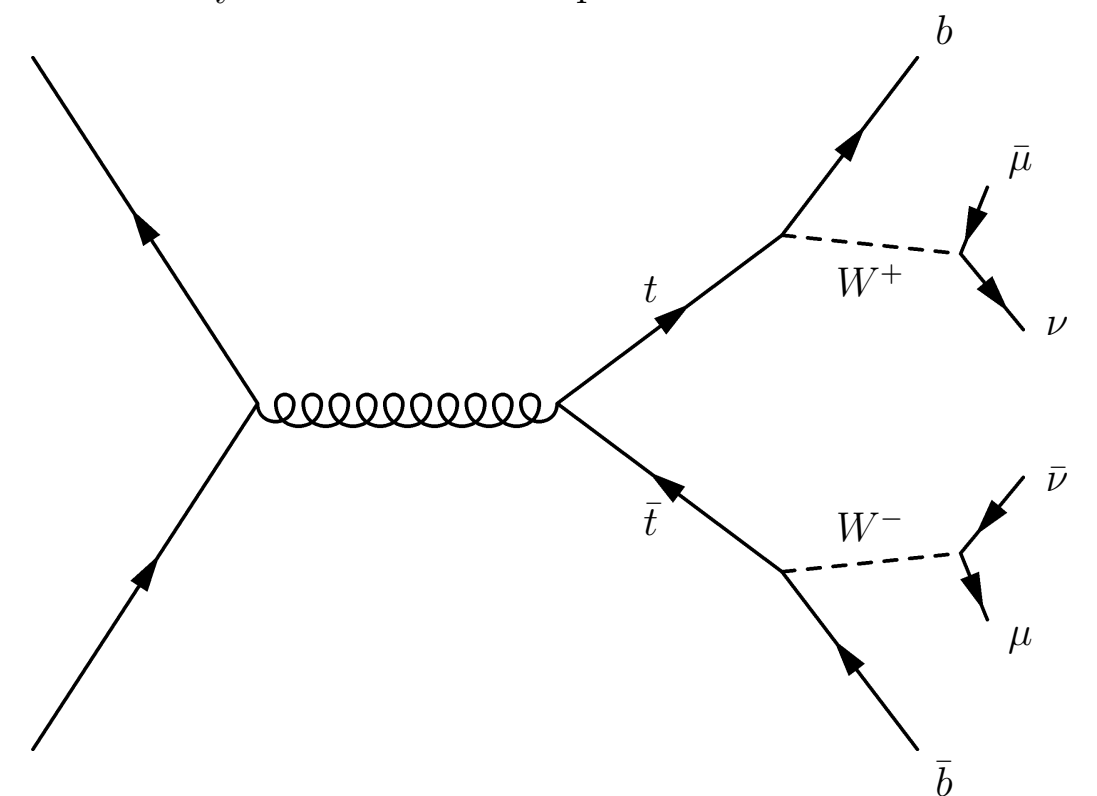

Figure 6.1.: Top quark pair production (through quark-antiquark annihilation) and decay

\subsection{Backgrounds}

The major backgrounds in the dimuon channel are due to $Z / \gamma^{*} \rightarrow \mu \bar{\mu}, Z / \gamma^{*} \rightarrow \tau \bar{\tau} \rightarrow \mu \bar{\mu} \nu \bar{\nu}$ and $W W \rightarrow \mu \bar{\mu} \nu \bar{\nu}$ (Fig. 6.2) events with two muons in their final states, and due to fake muons. Other backgrounds come from $W Z$ (Fig. 6.3) and $Z Z$ (Fig. 6.4) processes. The $\mathrm{Z}$ and diboson backgrounds are evaluated from Monte Carlo, the fake muon background is 

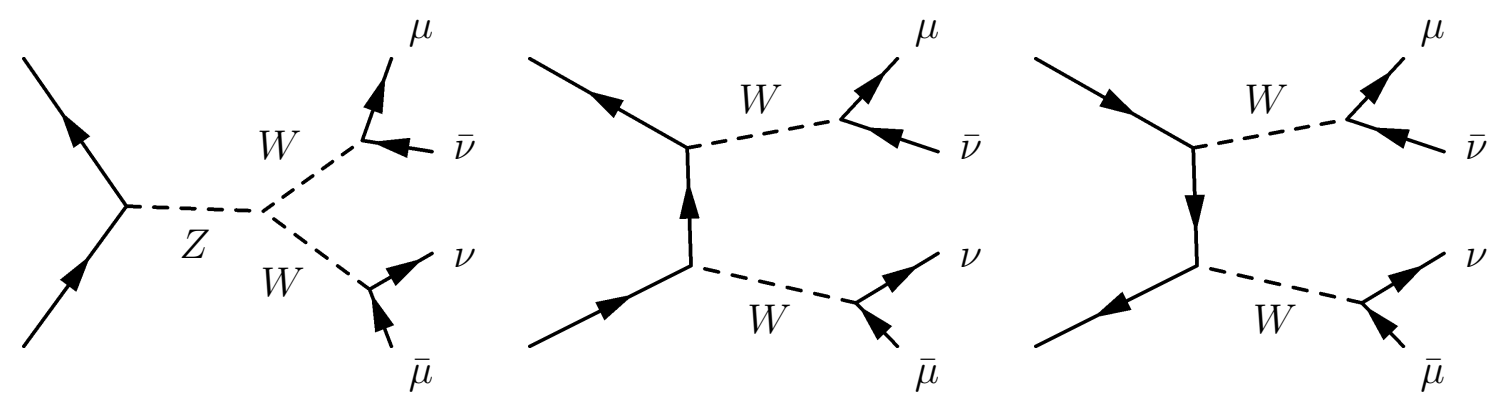

Figure 6.2.: $W$ boson pair production and decay

determined from the data sample as described in Sec. 6.3.6, $\tau$ lepton decays are performed with the TAUOLA package [84].
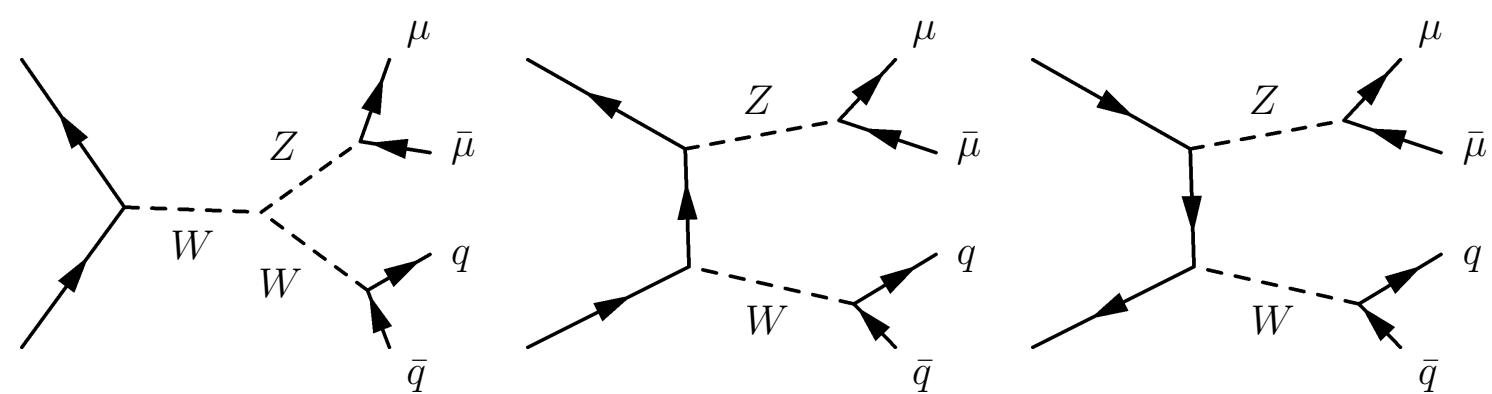

Figure 6.3.: $W Z$ boson production and decay
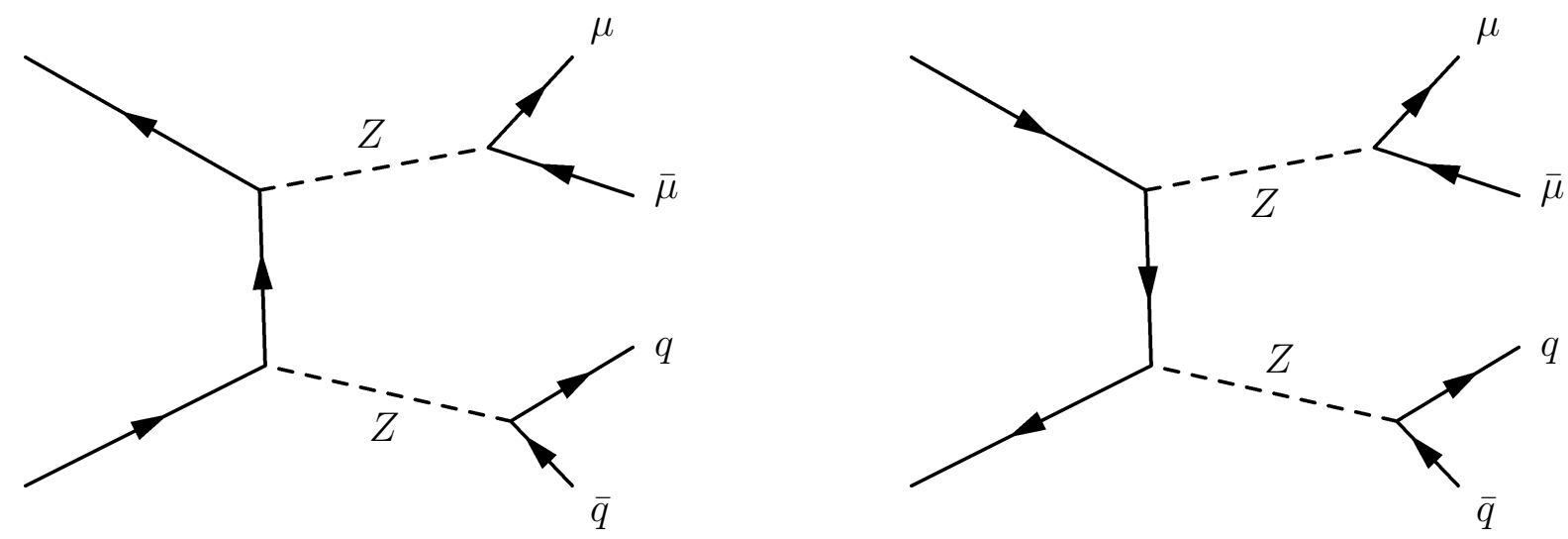

Figure 6.4.: $Z$ boson pair production and decay

The ALPGEN $Z$ Monte Carlo used is split into exclusive samples with zero to two light partons $(l p)$, and a three light parton inclusive sample (see Tab. 6.1). Additional samples of $Z+c \bar{c}+\mathrm{n} l p$ and $Z+b \bar{b}+\mathrm{n} l p$ were generated to gain on statistics. In these samples, the heavy flavor $(H F)$ contribution is already included in the matrix element as opposed to parton distribution and gluon splitting functions used by the shower evolution. To be able 
to combine them with the $Z+\mathrm{n} l p$ samples, the overlap in phase space has to be removed. This is done by heavy flavor removal [85]. In the $Z+\mathrm{n} l p$ samples, events with both $c \bar{c}$ and $b \bar{b}$ were removed, and in the $Z+b \bar{b}+\mathrm{n} l p$ samples events $c \bar{c}$ were removed. The event numbers before and after the heavy flavor removal are shown in tables 6.2 and 6.3 . As the heavy flavor removal decreases the cross section through removing parts of the phase space, this was corrected by multiplying the ALPGEN cross-section with the heavy flavor removal efficiency. The resulting cross sections are listed in the rightmost column of Tables 6.2 and 6.3 .

\begin{tabular}{|c|c|c|}
\hline Process & $\sigma_{L O} \times B R / p b$ & $\sigma_{N N L O / N L O} \times B R / p b$ \\
\hline$Z / \gamma^{*} \rightarrow \mu \bar{\mu}(15 \mathrm{GeV}-60 \mathrm{GeV})$ & 354.22 & $354.22 \times 1.33$ \\
\hline$Z / \gamma^{*} \rightarrow \mu \bar{\mu}(60 \mathrm{GeV}-130 \mathrm{GeV})$ & 193.79 & $256.6_{-12.0}^{+5.1}$ \\
\hline$Z / \gamma^{*} \rightarrow \mu \bar{\mu}(130 \mathrm{GeV}-250 \mathrm{GeV})$ & 1.34 & $1.34 \times 1.33$ \\
\hline$Z / \gamma^{*} \rightarrow \tau \bar{\tau}(15 \mathrm{GeV}-60 \mathrm{GeV})$ & 355.36 & $355.36 \times 1.33$ \\
\hline$Z / \gamma^{*} \rightarrow \tau \bar{\tau}(60 \mathrm{GeV}-130 \mathrm{GeV})$ & 194.32 & $256.6_{-12.0}^{+5.1}$ \\
\hline$Z / \gamma^{*} \rightarrow \tau \bar{\tau}(130 \mathrm{GeV}-250 \mathrm{GeV})$ & 1.38 & $1.38 \times 1.33$ \\
\hline WW incl. PYTHIA & $9.47 \pm 0.09$ & $12 \pm 0.6($ scale $) \pm 0.3(P D F)$ \\
\hline WZ inc.l PYTHIA & $5.2 \pm 0.11$ & $3.68 \pm 0.222($ scale $) \pm 0.12(P D F)$ \\
\hline ZZ incl. PYTHIA & $0.198 \pm 0.003$ & $1.42 \pm 0.06($ scale $) \pm 0.05(P D F)$ \\
\hline
\end{tabular}

Table 6.1.: LO and NLO (Diboson)/NNLO $(Z)$ cross sections for the various background processes [86], [87]. The K-factor of 1.33 for $Z$ production is derived in the central mass bin using the LO ALPGEN cross-section and the NNLO prediction.

\section{$Z p_{T}$ Reweighting}

The $p_{T}$ distribution of the $Z$ boson is not properly described in the simulation of $Z \rightarrow \mu \bar{\mu}$ and $Z \rightarrow \tau \bar{\tau}$ events. We apply an additional event weight for $Z / \gamma^{*} \rightarrow \mu \bar{\mu}$ and $Z / \gamma^{*} \rightarrow \tau \bar{\tau}$ Monte Carlo events according to the following method [88. The $Z p_{T}$ distribution as calculated from reconstructed electrons is measured in both the dielectron data skim of the common samples group and in ALPGEN $Z \rightarrow e \bar{e}$ Monte Carlo samples. The ratio of the data and the Monte Carlo is fitted using the cumulative distribution function,

$$
\epsilon\left(p_{T}\right)=\frac{\epsilon_{0}}{2}\left(1+\frac{2}{\sqrt{\pi}} \int_{0}^{\frac{p_{T}-\mu}{\sigma \sqrt{2}}} e^{-t^{2}} d t\right)
$$

of a Gaussian with mean $\mu$ and variance $\sigma$. This is done for events with no, exactly one and two or more jets separately.

The $Z p_{T}$ reweighting constructed this way does not preserve normalization, i.e. the sum of the weights for the $Z$ MC does not need to be one, due to the fact that the LO ALPGEN Monte Carlo samples are compared to data. This is corrected by scaling the different $Z$ MC mass bins to hold the same number of events before and after reweighting. As a result, the $Z p_{T}$ reweighting includes a factor that absorbs the dependence of the 


\begin{tabular}{|c|c|c|c|c|c|}
\hline Process & $\begin{array}{l}\mathrm{N} \text { before } \\
\mathrm{HF} \text { skimming }\end{array}$ & $\begin{array}{l}\mathrm{N} \text { after } \\
\mathrm{HF} \text { skimming }\end{array}$ & Efficiency $\epsilon$ & Alpgen $\sigma / \mathrm{pb}$ & $\sigma \times \epsilon / \mathrm{pb}$ \\
\hline \multicolumn{4}{|c|}{$Z / \gamma^{*} \rightarrow \mu \bar{\mu}, 15 \mathrm{GeV}<M_{l \bar{l}}<60 \mathrm{GeV}$} & 386.02 & 354.22 \\
\hline$+0 l p$ excl. & 607071 & 560852 & $92.387 \%$ & 334.14 & 308.70 \\
\hline+1 lp excl. & 475931 & 423180 & $88.916 \%$ & 38.63 & 34.35 \\
\hline$+2 l p$ excl. & 191749 & 163310 & $85.169 \%$ & 10.15 & 8.65 \\
\hline$+3 l p$ incl. & 94431 & 76757 & $81.284 \%$ & 3.10 & 2.52 \\
\hline \multicolumn{4}{|c|}{$Z / \gamma^{*} \rightarrow \mu \bar{\mu}, 60 \mathrm{GeV}<M_{l \bar{l}}<130 \mathrm{GeV}$} & 195.33 & 187.82 \\
\hline$+0 l p$ excl. & 2339833 & 2279788 & $97.434 \%$ & 139.76 & 136.17 \\
\hline+1 lp excl. & 396953 & 375077 & $94.489 \%$ & 41.86 & 39.55 \\
\hline$+2 l p$ excl. & 198186 & 178071 & $89.85 \%$ & 10.38 & 9.33 \\
\hline$+3 l p$ incl. & 291266 & 242080 & $83.11 \%$ & 3.33 & 2.77 \\
\hline \multicolumn{4}{|c|}{$Z / \gamma^{*}+c \bar{c} \rightarrow \mu \bar{\mu}, 60 \mathrm{GeV}<M_{l \bar{l}}<130 \mathrm{GeV}$} & 4.53 & 4.53 \\
\hline$+c \bar{c}+0 l p$ excl. & $47125^{\text {cu }}$ & 47125 & $100 \%$ & 3.05 & 3.05 \\
\hline$+c \bar{c}+1$ lp excl. & 42776 & 42776 & $100 \%$ & 1.07 & 1.07 \\
\hline$+c \bar{c}+2 l p$ incl. & 23317 & 23317 & $100 \%$ & 0.41 & 0.41 \\
\hline \multicolumn{4}{|c|}{$Z / \gamma^{*}+b \bar{b} \rightarrow \mu \bar{\mu}, 60 \mathrm{GeV}<M_{l \bar{l}}<130 \mathrm{GeV}$} & 1.52 & 1.44 \\
\hline$+b \bar{b}+0 l p$ excl. & $273184^{\iota}$ & 266953 & $97.719 \%$ & 1.0 & 0.98 \\
\hline$+b \bar{b}+1 l p$ excl. & 50585 & 48052 & $94.993 \%$ & 0.37 & 0.35 \\
\hline$+b \bar{b}+2 l p$ incl. & 24211 & 21627 & $89.327 \%$ & 0.15 & 0.13 \\
\hline \multicolumn{4}{|c|}{$Z / \gamma^{*} \rightarrow \mu \bar{\mu}, 130 \mathrm{GeV}<M_{l \bar{l}}<250 \mathrm{GeV}$} & 1.42 & 1.34 \\
\hline$+0 l p$ excl. & 102912 & 100603 & $97.756 \%$ & 0.90 & 0.88 \\
\hline+1 lp excl. & 95836 & 90775 & $94.719 \%$ & 0.36 & 0.34 \\
\hline$+2 l p$ excl. & 96737 & 86490 & $89.407 \%$ & 0.10 & 0.09 \\
\hline$+3 l p$ incl. & 579561 & 481545 & $83.088 \%$ & 0.06 & 0.05 \\
\hline \multirow{2}{*}{\multicolumn{5}{|c|}{$\begin{array}{l}Z / \gamma^{*} \rightarrow \mu \bar{\mu}, 60 \mathrm{GeV}<M_{l \bar{l}}<130 \mathrm{GeV} \text { total } \\
Z / \gamma^{*} \rightarrow \mu \bar{\mu} \text { total }\end{array}$}} & 193.79 \\
\hline & & & & & 549.35 \\
\hline
\end{tabular}

Table 6.2.: The number of events in ALPGEN $Z \rightarrow \mu \bar{\mu}$ MC samples before and after heavy flavor skimming, and their respective cross sections. See Sec. 5.2.2 for the explanation of $\mathrm{n} l p$, exclusive and inclusive.

$\mathrm{K}$-factor on the mass window of the $Z$ Monte Carlo sample. Thus, a constant K-factor is applied to the $Z$ MC samples. After reweighting, the $Z p_{T}$ distributions in MC and data agree reasonably well, see Fig. 6.5, as does the jet multiplicity.

In the next-to-leading order (and higher order) calculations the relative contribution of $Z+$ light and $Z+$ heavy jets is different from the leading order calculation. Thus, an additional $\mathrm{K}$-factor of 1.25 is applied to the $Z+\mathrm{HF}$ samples [89] before scaling the $Z \mathrm{MC}$ to the number of expected events as calculated with the luminosity of our data set.

The diboson samples are scaled to the number of events expected in the data luminosity, $\sigma_{N L O} \times \mathcal{L}$. 


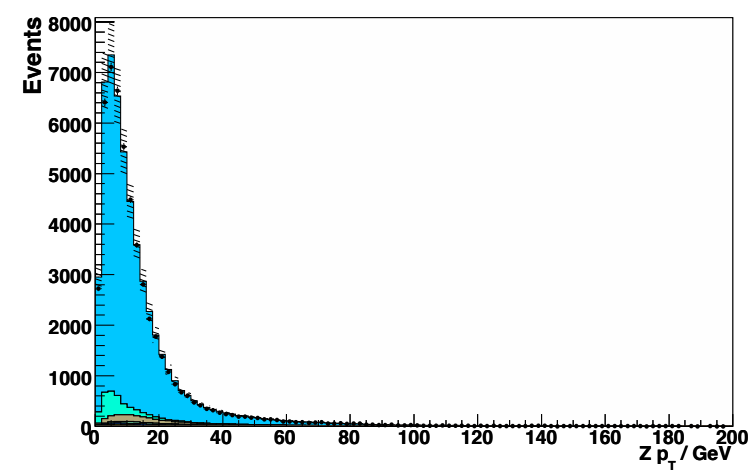

(a) $Z p_{T}$ distribution in the zero jets inclusive bin.

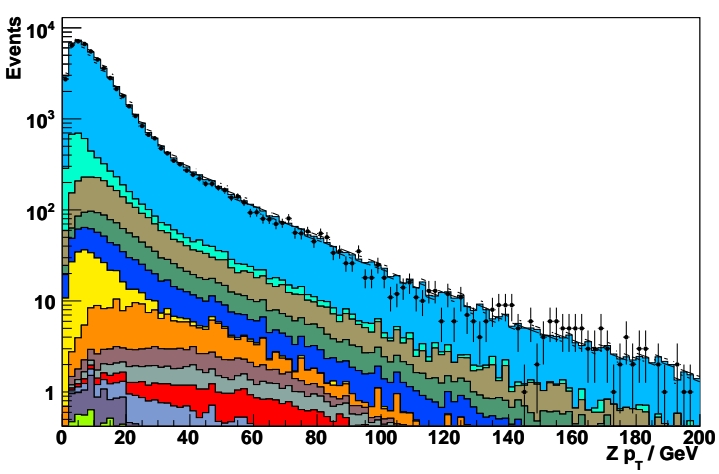

(b) $Z p_{T}$ distribution in the zero jets inclusive bin with logarithmic scale

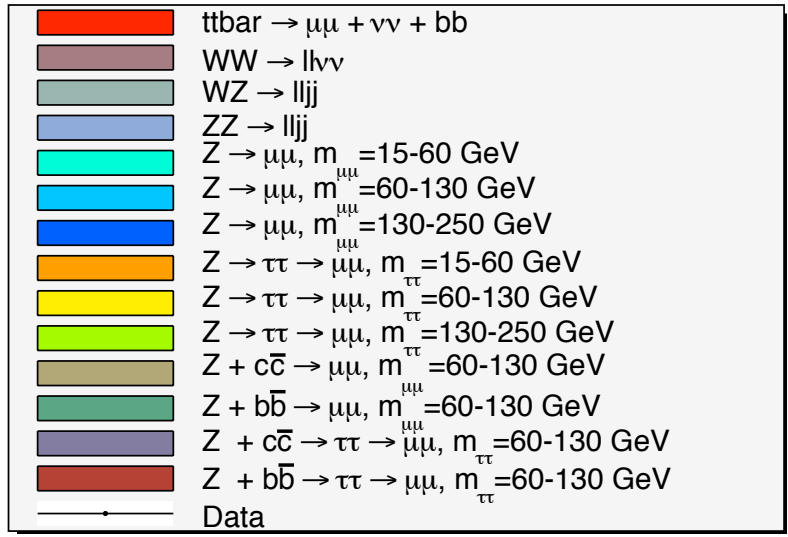

(d) Jet multiplicity.

Figure 6.5.: The $Z p_{T}$ distribution calculated from the muon pair momenta and jet multiplicity after $Z p_{T}$ reweighting. Points represent data. 


\begin{tabular}{|c|c|c|c|c|c|}
\hline Process & $\begin{array}{l}\mathrm{N} \text { before } \\
\text { HF skimming }\end{array}$ & $\begin{array}{l}\mathrm{N} \text { after } \\
\mathrm{HF} \text { skimming }\end{array}$ & Efficiency $\epsilon$ & Alpgen $\sigma / \mathrm{pb}$ & $\sigma \times \epsilon / \mathrm{pb}$ \\
\hline \multicolumn{4}{|c|}{$Z / \gamma^{*} \rightarrow \tau \bar{\tau}, 15 \mathrm{GeV}<M_{\bar{l}}<60 \mathrm{GeV}$} & 387.30 & 355.36 \\
\hline$+0 l p$ excl. & 579281 & 535103 & $92.374 \%$ & 335.43 & 309.85 \\
\hline+1 lp excl. & 484201 & 430829 & $88.977 \%$ & 38.54 & 34.29 \\
\hline$+2 l p$ excl. & 196089 & 166837 & $85.083 \%$ & 10.27 & 8.74 \\
\hline$+3 l p$ incl. & 94043 & 76321 & $81.155 \%$ & 3.06 & 2.48 \\
\hline \multicolumn{4}{|c|}{$Z / \gamma^{*} \rightarrow \tau \bar{\tau}, 60 \mathrm{GeV}<M_{\bar{l}}<130 \mathrm{GeV}$} & 195.90 & 188.35 \\
\hline$+0 l p$ excl. & 2349066 & 2288791 & $97.434 \%$ & 139.74 & 136.15 \\
\hline$+1 l p$ excl. & 588415 & 556291 & $94.541 \%$ & 42.01 & 39.72 \\
\hline$+2 l p$ excl. & 197712 & 177619 & $89.837 \%$ & 10.8 & 9.7 \\
\hline$+3 l p$ incl. & 281634 & 234122 & $83.13 \%$ & 3.35 & 2.78 \\
\hline \multicolumn{4}{|c|}{$Z / \gamma^{*}+c \bar{c} \rightarrow \tau \bar{\tau}, 60 \mathrm{GeV}<M_{\bar{l}}<130 \mathrm{GeV}$} & 4.51 & 4.51 \\
\hline$+c \bar{c}+0 l p$ excl. & 39295 & 39295 & $100 \%$ & 3.05 & 3.05 \\
\hline$+c \bar{c}+1 l p$ excl. & 43491 & 43491 & $100 \%$ & 1.08 & 1.04 \\
\hline$+c \bar{c}+2 l p$ incl. & 20930 & 20930 & $100 \%$ & 0.42 & 0.42 \\
\hline \multicolumn{4}{|c|}{$Z / \gamma^{*}+b \bar{b} \rightarrow \tau \bar{\tau}, 60 \mathrm{GeV}<M_{l \bar{l}}<130 \mathrm{GeV}$} & 1.52 & 1.46 \\
\hline$+b \bar{b}+0 l p$ excl. & $95209^{\circ}$ & 93013 & $97.693 \%$ & 1.00 & 0.98 \\
\hline$+b \bar{b}+1 l p$ excl. & 191992 & 182386 & $94.997 \%$ & 0.37 & 0.35 \\
\hline$+b \bar{b}+2 l p$ incl. & 96786 & 86872 & $89.575 \%$ & 0.15 & 0.13 \\
\hline \multicolumn{4}{|c|}{$Z / \gamma^{*} \rightarrow \tau \bar{\tau}, 130 \mathrm{GeV}<M_{l \bar{l}}<250 \mathrm{GeV}$} & 1.44 & 1.38 \\
\hline$+0 l p$ excl. & 101887 & 99596 & $97.751 \%$ & 0.91 & 0.89 \\
\hline+1 lp excl. & 95045 & 89944 & $94.633 \%$ & 0.37 & 0.35 \\
\hline$+2 l p$ excl. & 89806 & 80372 & $89.495 \%$ & 0.1 & 0.09 \\
\hline$+3 l p$ incl. & 86867 & 71102 & $81.852 \%$ & 0.06 & 0.05 \\
\hline \multirow{2}{*}{\multicolumn{5}{|c|}{$\begin{array}{l}Z / \gamma^{*} \rightarrow \tau \bar{\tau}, 60 \mathrm{GeV}<M_{l \bar{l}}<130 \mathrm{GeV} \text { total } \\
Z / \gamma^{*} \rightarrow \tau \bar{\tau} \text { total }\end{array}$}} & 194.32 \\
\hline & & & & & 551.06 \\
\hline
\end{tabular}

Table 6.3.: The number of events in ALPGEN $Z \rightarrow \tau \bar{\tau}$ MC samples before and after heavy flavor skimming, and their respective cross sections. See Sec. 5.2.2 for the explanation of $n l p$, exclusive and inclusive.

\subsection{Dimuon Event Selection and Data/Monte Carlo Comparison}

\subsubsection{Object Identification}

\section{Muon Selection}

In the analyses presented here, we select three types of muons. High- $p_{T}$ non-isolated muons are selected for JES corrections of jets with semileptonic decays, muons without isolation requirements for the $\mathbb{E}_{T}$ computation, and finally high- $p_{T}$, isolated "signal muons" from the $\mathrm{W}$ decay in the top quark decay chain.

Jet energy scale $(J E S)$ corrections for semileptonic decays were derived with respect to muons of "medium" quality with NSeg=3, "Met" track matching and $\left|\eta_{\text {det }}\right|<2.0$, thus we select muons with these characteristics for the JES. However, only the muon with the 
highest momentum in the direction of the jet under consideration is used for the JES correction.

Muons that pass the "signal" muon selection are of "loose" quality and have a "medium" track match. There is no cut on the muon type parameter NSeg. Isolation is required by cutting on $\Delta R(\mu, j e t)>0.5$ and $p_{T}:\left|\sum_{\text {tracks }} p_{T} / p_{T}(\mu)\right|<0.1$ (cf. 4.3.3). The muons have to be inside the muon system, $\left|\eta_{\text {det }}\right|<2.0$ and have to pass a cut on $p_{T}, p_{T}>15 \mathrm{GeV}$.

Muons for $E_{T}$ computation are much like "signal muons" except that there is no isolation requirement, and muons already used in the JES jet correction are not taken into account. There is no cut on $\left|\eta_{\text {det }}\right|$ or $p_{T}$ of these muons.

All three muon selections include timing cuts against cosmic muons. The different muon requirements are summarized in Tab. 6.4.

\begin{tabular}{|c|c|c|c|c|}
\hline Muons for & Quality & Track match & Isolation & Additional Cuts \\
\hline JES & $\begin{array}{l}\text { medium, } \\
N \text { seg }==3\end{array}$ & $\chi^{2}<100$ & none & $\left|\eta_{\text {det }}\right|<2.0$ \\
\hline $\begin{array}{l}E_{T} \text { correction } \\
\text { computation }\end{array}$ & $\begin{array}{l}\text { loose, } \\
\text { Nseg }>=0\end{array}$ & $\chi^{2}<100$ & none & $\begin{array}{l}\text { veto muons used } \\
\text { for JES }\end{array}$ \\
\hline "signal" & $\begin{array}{l}\text { loose, } \\
N \text { seg }>=0\end{array}$ & $\begin{array}{l}\chi^{2}<4 \\
d c a_{S M T}<0.02, \\
d c a_{n o S M T}<0.2\end{array}$ & $\begin{array}{l}\Delta R(\mu, j e t)>0.5 \\
\sum_{\text {tracks }} p_{T} / p_{T}(\mu)<0.1\end{array}$ & $\begin{array}{l}\left|\eta_{\text {det }}\right|<2.0 \\
p_{T}>20 \mathrm{GeV} \text { (leading) } \\
p_{T}>15 \mathrm{GeV}\end{array}$ \\
\hline
\end{tabular}

Table 6.4.: Overview of the different muon types used in this analysis.

\section{Electron Selection}

To be orthogonal to other $t \bar{t} \rightarrow l l$ channel cross section measurements, electrons are selected the same way as in the dielectron and electron-muon cross section analyses. A veto on these electrons is applied, i.e. we reject events with one or more of these electrons. The $t \bar{t} \rightarrow e e$ and $t \bar{t} \rightarrow e \mu$ cross section analyses select electrons of type "top_tight" with $p_{T}>15 \mathrm{GeV}$ that are either inside the central calorimeter $\left(\left|\eta_{\text {det }}\right|<1.1\right)$ or one of the endcap calorimeters $\left(1.5<\left|\eta_{\text {det }}\right|<2.5\right)$.

Second, we select "top_loose" electrons with $p_{T}>15 \mathrm{GeV}$ to detect electron-jet overlaps. Jets overlapping with these electrons will be removed during jet selection.

The last type of electrons is selected for $E_{T}$ correction computation. It consists of "d0correct" type electrons with $\left|\eta_{\text {det }}\right|<2.5$.

The different electron definitions are summarized in Tab. 6.5.

\section{Jet Selection}

The selection of jets begins with the removal of electron-jet overlaps and the application of the jet energy scale (JES). This is done both for jets in our data sample and jets in the different Monte Carlo samples. Next, we select jets that pass the jet ID criteria of a "good" jet (cf. Sec. 4.5) and require $\left|\eta_{\text {det }}\right|<2.5$. All jets must pass a $p_{T}>20 \mathrm{GeV}$ cut. 


\begin{tabular}{c|l} 
Electrons for & Selection \\
\hline \hline electron veto & "top_tight": Isolation $<0.15$, EM fraction $>0.9, \mathrm{HMx} 7<50$, \\
& $\chi^{2}$ from track match $>0$, Track $p_{T}>5 \mathrm{GeV}$, Electron likelihood $>0.85 ;$ \\
& $\left|\eta_{\text {det }}\right|<1.1$ or $1.5<\left|\eta_{\text {det }}\right|<2.5 ; p_{T}>15 \mathrm{GeV}$ \\
\hline electron-jet & "top_loose": Isolation $<0.15$, EM fraction $>0.9, \mathrm{HMx} 7<50$, \\
overlap removal & $\chi^{2}$ from track match $>0$, Track $p_{T}>5 \mathrm{GeV} ; p_{T}>15 \mathrm{GeV}$ \\
\hline$E_{T}$ correction & "d0correct": Isolation $<0.15$, EM fraction $>0.9, \mathrm{HMx} 8<10000$, \\
computation & $p_{T}>5 \mathrm{GeV},\left|\eta_{\text {det }}\right|<2.5$
\end{tabular}

Table 6.5.: Overview of the different electron types used in this analysis.

Before selecting jets in the Monte Carlo samples, we must apply corrections for reconstruction efficiency, jet energy resolution and energy offset.

Jets for the missing transverse energy calculation are selected the same way except there is no cut on $p_{T}$ or $\left|\eta_{\text {det }}\right|$.

\subsubsection{Event Preselection and Corrections}

The events have to pass the standard data quality requirements to ensure correct functionality of all detector subsystems.

Monte Carlo corrections applied at this point are a reweighting depending on the instantaneous luminosity and a reweighting of Monte Carlo events depending on the instantaneous luminosity and run range of the event overlaid by DØSIM and the primary vertex $\mathrm{z}$ position. The former reweighting improves the agreement of the instantaneous luminosity profile in data and $\mathrm{MC}$, the latter reweighting brings the longitudinal shape of the luminous beam region in data and $\mathrm{MC}$ into agreement.

Muon $p_{T}$ is smeared in the Monte Carlo samples so the $p_{T}$ resolution matches data.

Next, muons for the JES correction are selected as well as electrons for the jet-electron overlap removal. Jets overlapping with any of the selected electrons are flagged for later removal, jet energy scale corrections are applied and jets selected as described above; Jet shifting, smearing and removal is applied to jets in the MC samples. With the selected (and corrected in the case of MC) jets at hand, we can reweight the events in $Z$ Monte Carlo samples to improve agreement between the $Z$ boson $p_{T}$ spectrum in data and these Monte Carlo samples. The parameters for this reweighting are depending on the number of jets in the event, none, exactly one or greater or equal to two.

To combine the measured cross section with the cross section measured in other dilepton channels, we select and put a veto on standard tight electrons as described above. The event selection is thus orthogonal to the other dilepton selections.

Next, two "signal" muons and two jets are required, the leading muon satisfying $p_{T}^{\text {lead }}>$ $20 \mathrm{GeV}$ and the leading jet satisfying $p_{T}^{\text {lead }}>30 \mathrm{GeV}$. Muon selection and trigger efficiency corrections are applied to MC events. In the last cut of the preselection we select events with exactly one primary vertex. The calculation of the $\not_{T}$ concludes the preselection. 


\subsubsection{Background Rejection}

After the event preselection, we are left with events that contain two well identified muons from the primary vertex and no tight electron. To further suppress the fake muon background from QCD and $W+b \bar{b}$ or $W+c \bar{c}$, the two leading muons are required to have opposite charge. The leading muon $p_{T}$ has to be greater than $20 \mathrm{GeV}$. The background from $J / \Psi$ and $\Upsilon$ resonances is suppressed by removing events with $M_{\mu \bar{\mu}}<30 \mathrm{GeV}$. To reduce the background from $Z / \gamma^{*}+$ jets $\rightarrow l \bar{l}+$ jets the leading jet has to pass $p_{T}>30 \mathrm{GeV}$. In addition, the jet multiplicity has to be greater than or equal to two. This reduces the $\mathrm{Z}$ as well as the diboson background considerably. However, due to the large production cross section and branching fraction, the $Z \rightarrow \mu \bar{\mu}$ background is still dominant.

$Z \rightarrow \mu \bar{\mu}$ events have no "real" $E_{T}$. Thus requiring $\mathbb{E}_{T}>40 \mathrm{GeV}$ and missing transverse energy from neutrinos through the $E_{T}$ significance, the $\mathrm{Z}$ background can be reduced substantially.

\subsubsection{Missing Transverse Energy Significance}

A cut on the reconstructed mass of the dimuon system is a powerful method of rejecting the dominant resonant $Z$ background.

However, the cut on the invariant mass would reject a large number of signal events. This is due to the muon transverse momentum resolution that does not allow a narrow $Z$ mass window cut. Hence, a different approach to reject $Z / \gamma^{*} \rightarrow \mu \bar{\mu}$ and $Z / \gamma^{*} \rightarrow \tau \bar{\tau}$ background is chosen.

The probability distribution $p\left(E_{T}\right)$ that the measured $E_{T}$ is due to a fluctuation within the resolution of the measured objects is estimated [90]. The starting point to derive this distribution is the probability $p\left(E_{T}\right)$ that physics objects with energy $E_{T}$ were measured as having an energy of $E_{T}^{\text {meas }}$, which is given by

$$
p\left(E_{T}\right)=\mathcal{G}\left(E_{T}, E_{T}^{\text {meas }}, \sigma\left(E_{T}^{\text {meas }}, \eta\right)\right)
$$

where $\mathcal{G}\left(E_{T}, E_{T}^{\text {meas }}, \sigma\left(E_{T}^{\text {meas }}, \eta_{j}\right)\right)$ is a Gaussian distribution with mean $E_{T}^{\text {meas }}$ and width $\sigma_{j}=\sigma\left(E_{T}^{\text {meas }}, \eta\right)$. Thus, the variation with respect to the mean value can be written as $p\left(\Delta E_{T}\right)=p\left(E_{T}-E_{T}^{\text {meas }}\right)=\mathcal{G}\left(E_{T}-E_{T}^{\text {meas }}, 0, \sigma\left(E_{T}^{\text {meas }}, \eta\right)\right)$. The probability for a change in missing transverse energy, $p\left(\Delta E_{T}\right)$ can now be expressed as the convolution of the probability distributions $p\left(\Delta E_{T}^{i}\right)$ for the variation of the transverse energy of the $i$ th object. Thus, the probability distribution for $\Delta E_{T}$ can be expressed as

$$
p\left(\Delta \#_{T}\right)=N\left(E_{T}^{m e a s}-E_{T}, 0, \sqrt{\sum_{i=0}^{N} \sigma_{i}^{2}}\right),
$$

where $\mathbb{E}_{T}^{\text {meas }}$ is the $\mathbb{E}_{T}$ measured in the event and $\sigma_{i}$ the transverse energy resolution of the $i$ th object in the event.

The probability distribution for the projection of the missing transverse energy in the direction of the measured $E_{T}$ can be obtained the same way by computing the probability distributions for the energies $E_{i}$ projected along the direction of the measured transverse energy, $\vec{a}$. The energy resolution $\sigma_{j}$ is now replaced by $\sigma_{j} \cdot \cos (\vec{j}, \vec{a})$, where $\cos (\vec{j}, \vec{a})$ is the cosine of the angle between the direction of the missing transverse energy and the direction of the energy of the $j$ th object in the event in the transverse plane. 


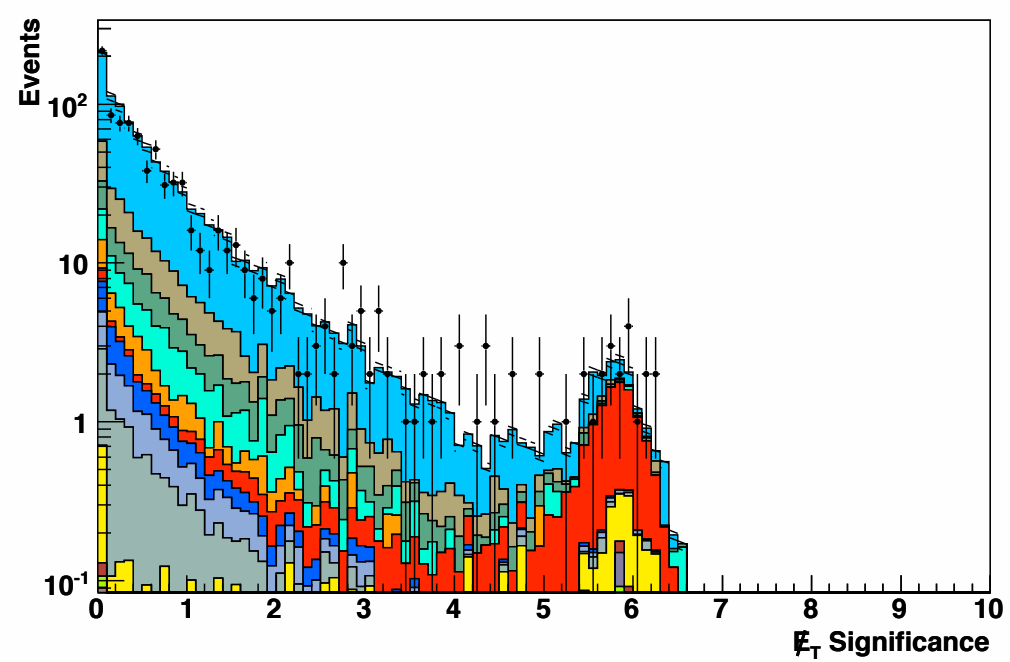

Figure 6.6.: The $E_{T}$ significance distribution after cut 15 of Section 6.3.5.

Finally, the likelihood

$$
L=\log \frac{p\left(E_{T}=E_{T}^{\text {meas }}\right)}{p\left(\not_{T}=0\right)}
$$

can be defined with the above definition of the missing transverse energy probability distribution.

The algorithm described in ([91], [90]) was updated to contain the latest object resolutions as they are an important input to the $\mathbb{E}_{T}$ significance algorithm. In addition, the unclustered scalar $E_{T}$ resolution is needed.

The $E_{T}$ significance likelihood of $Z$ events (containing no neutrinos) should be peaking at 0 . On the other hand, for events with "real" $E_{T}$ from $W \rightarrow l \nu_{l}$ decays the $E_{T}$ significance likelihood is supposed to be different from 0, peaking between 5 and 7 depending on the event topology in question. Thus, the missing transverse energy significance provides good separation between the dominant $Z / \gamma^{*} \rightarrow l \bar{l}$ background and the $t \bar{t}$ signal, c.f. Fig. 6.6).

\subsubsection{Event Selection Summary and Cutflow}

In the following a list of all event selection criteria in order of their application is given.

1. Luminosity block selection, i.e. removal of bad luminosity block numbers;

2. The calorimeter event quality variable described in the note [92] was required to be good, efficiencies for this selection have been determined in [93];

3. Luminosity Reweighting: correct Monte Carlo to match the data luminosity distribution

4. Beamshape Reweighting: correct Monte Carlo based on the z position of the primary vertex

5. Select jets according to the following standard procedure: 
a) for MC jets, the following corrections as implemented in caf_mc_util p18-br- 7 r are first applied:

i. jet $p_{T}$ smearing using shifting, smearing $(S S R)$ and removal procedure described in Sec. 5.2.6;

ii. jet $p_{T}$ shifting using SSR procedure;

iii. jet reconstruction "turn-on" efficiency correction using SSR procedure;

iv. jet reconstruction "plateau" efficiency correction using the jet ID group procedure;

b) The jet $p_{T}$ (corrected with jet energy scale including muon corrections) is greater than $20 \mathrm{GeV}$;

c) The jet $p_{T}$ (corrected with jet energy scale without muon corrections) is greater than $15 \mathrm{GeV}$;

d) the absolute value of the jet detector $\eta$ is less than 2.5;

e) The EM jet fraction is in the range $0.05-0.95$;

f) The jet coarse hadronic fraction is less than 0.4;

g) The reconstructed jet must be confirmed by the L1 trigger readout;

h) No requirement on the jet multiplicity

6. $Z p_{T}$ reweighting is applied depending on the number of selected jets.

7. Veto on any standard tight electron:

a) $\left|\eta_{\text {calo }}\right|<2.5$ and exclusion of the intercryostat detector region $\left(\left|\eta_{\text {calo }}\right|<1.1\right.$ or $\left|\eta_{\text {calo }}\right|>$ $1.5)$

b) High energy fraction in the EM part of the calorimeter: $f_{E M}>0.9$;

c) Isolated EM cluster: iso < 0.15;

d) Shower shape cut: $\chi_{h m x 7}^{2}<50$;

e) Electron likelihood $>0.85$;

f) $p_{T}>15 \mathrm{GeV}$;

g) One track matched with $E / p$ and track $p_{T}>5 \mathrm{GeV}$;

8. Standard loose muon selection with medium track match and isolation requirements:

a) Outside the bottom hole of the muon detector;

b) $\left|\eta_{\text {det }}\right|<2$;

c) Timing cuts against cosmic muons;

d) Matched to a medium central track, with the following quality criteria on the matched track:

i. $\chi_{t r k}^{2}<4$;

ii. $|D C A|<0.02 \mathrm{~cm}$ for tracks with SMT hits; and $|D C A|<0.2 \mathrm{~cm}$ for tracks without SMT hits; 
e) $p_{T}>15 \mathrm{GeV}$. For $\mathrm{MC}$ events, this is the smeared muon $p_{T}$ according to the data/MC difference in muon momentum resolution. The muon smearing as implemented in caf_mc_util p18-br-777 is used.

f) Isolation in the tracker (etTrkConeScaledMin $<0.1)$. The isolation is scaled by the muon $p_{T}$. Calorimeter isolation is achieved by requiring $\Delta R($ jets, $\mu)>0.5$.

g) $N_{\text {muons }} \geq 2$;

h) For MC events, the muon selection efficiency is corrected for the loose muon as well as for the medium track data/MC efficiency differences using standard muon ID correction factors as described in [59];

9. Standard vertex selection:

a) $\left|z_{P V}\right|<60 \mathrm{~cm}$

b) Number of tracks associated with the primary vertex greater or equal to 3;

c) Distance between the selected muon $z$ at $R=0$ and the primary vertex $z$ : $\left|z_{\text {muon }}-z_{P V}\right|<1 \mathrm{~cm}$

10. Trigger selection:

a) One single muon trigger listed in Tab. 5.3 fired;

b) The offline muons and tracks are asked to be matched with the corresponding L1, L2 and L3 objects to be consistent with the trigger efficiency calculation in data;

c) For MC, events have been reweighed according to the calculated trigger efficiency measured in data;

11. The leading jet $p_{T}$ is greater than $30 \mathrm{GeV}$;

12. The leading muon $p_{T}$ is greater than $20 \mathrm{GeV}$;

13. Choose the leading opposite sign muon pair;

14. The invariant mass of the leading muon pair has to be greater than $30 \mathrm{GeV}$;

15. Require at least two jets: $N_{\text {jets }} \geq 2$.

16. $E_{T}>40 \mathrm{GeV}$

17. $E_{T}$ significance likelihood $>5$

The efficiencies of the selection are shown in Table 6.6 for both the $t \bar{t} \rightarrow \mu \bar{\mu}$ events and for the complete Run IIa high- $p_{T}$ dimuon data set. The correction factors for the individual object identification are shown as well. The muon identification corrections are calculated as the ratio of efficiencies computed in data (using $Z \rightarrow \mu \bar{\mu}$ ) with the efficiencies computed in MC both measured by the muon ID group [59]. They have a statistical error below . $1 \%$ and are parametrized as a function of $(\eta, \phi)$ for the muon ID and as a function $\left(z_{\text {vertex }}, \eta_{C F T}\right)$ for the tracking effciency. The "overall efficiency correction" corresponds to the product of the individual scale factors (for trigger, muon identification and isolation, 


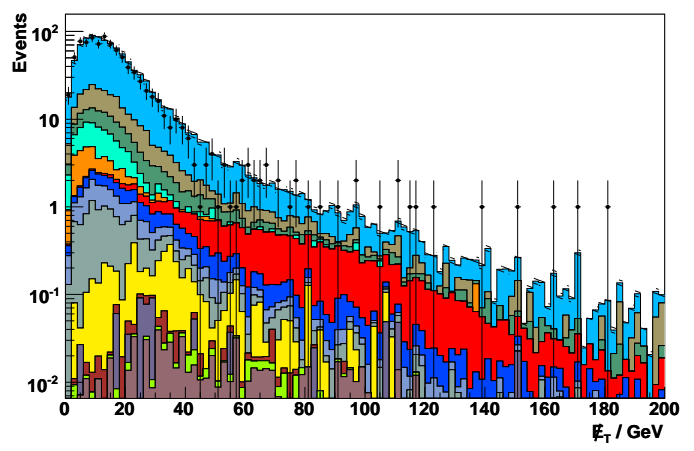

(a) $E_{T}$ distribution after the 2 jet selection.

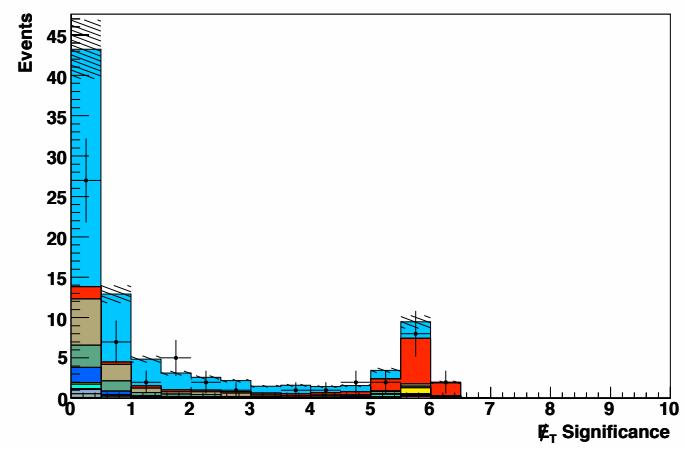

(b) $E_{T}$ significance distribution after the $\mathbb{E}_{T}$ cut.

Figure 6.7.: Distributions of $E_{T}$ on the left and $E_{T}$ significance on the right hand side illustrating the data and $\mathrm{MC}$ disagreement after the $E_{T}>\mathrm{GeV}$ cut.

and muon-track matching). The efficiency corrections for the primary vertex and opposite charge muon pair selection are discussed below.

The dependence of the selection efficiency on the top quark mass has been studied using signal Monte Carlo samples with $m_{\text {top }}$ between 160 and $185 \mathrm{GeV}$. The signal efficiency $\epsilon_{t \bar{t}}$ and the cross section calculated are given in table 6.11.

\section{Data and MC disagreement after the $E_{T}$ cut}

The overall expected main background as well as the observed and expected signal yield for the $t \bar{t} \rightarrow \mu \mu$ channel is summarized in Tab. 6.16. However, there is a discrepancy after the cut on transverse missing energy. This is due to a disagreement in data and Monte Carlo in the intermediate, $40 \mathrm{GeV}<E_{T}<70 \mathrm{GeV}$, region as shown on the left hand side of Fig. 6.7). A comparison with the $E_{T}$ significance plot (right hand side on the bottom of Fig. 6.7) shows that these events have a small significance of less than 2, which indicates that the measured $E_{T}$ is due to energy mismeasurement of one or more objects in the event. This is supported by the invariant mass plot, Fig. 6.20, that shows an excess of Monte Carlo over data in the region with the largest $Z \rightarrow \mu \bar{\mu}$ contribution. The latter is suppressed by the $E_{T}$ significance cut, and the Monte Carlo over data agreement is regained. 

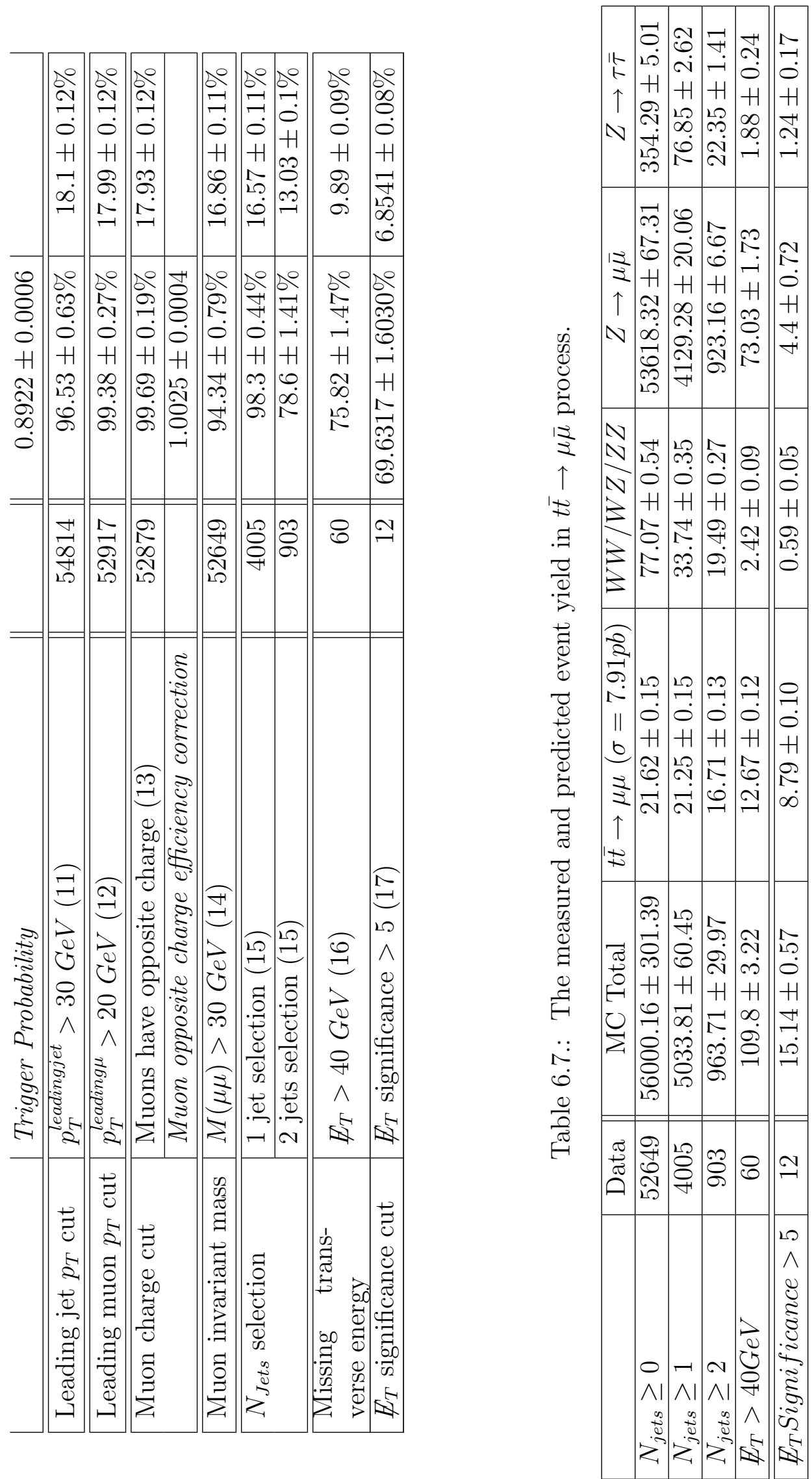

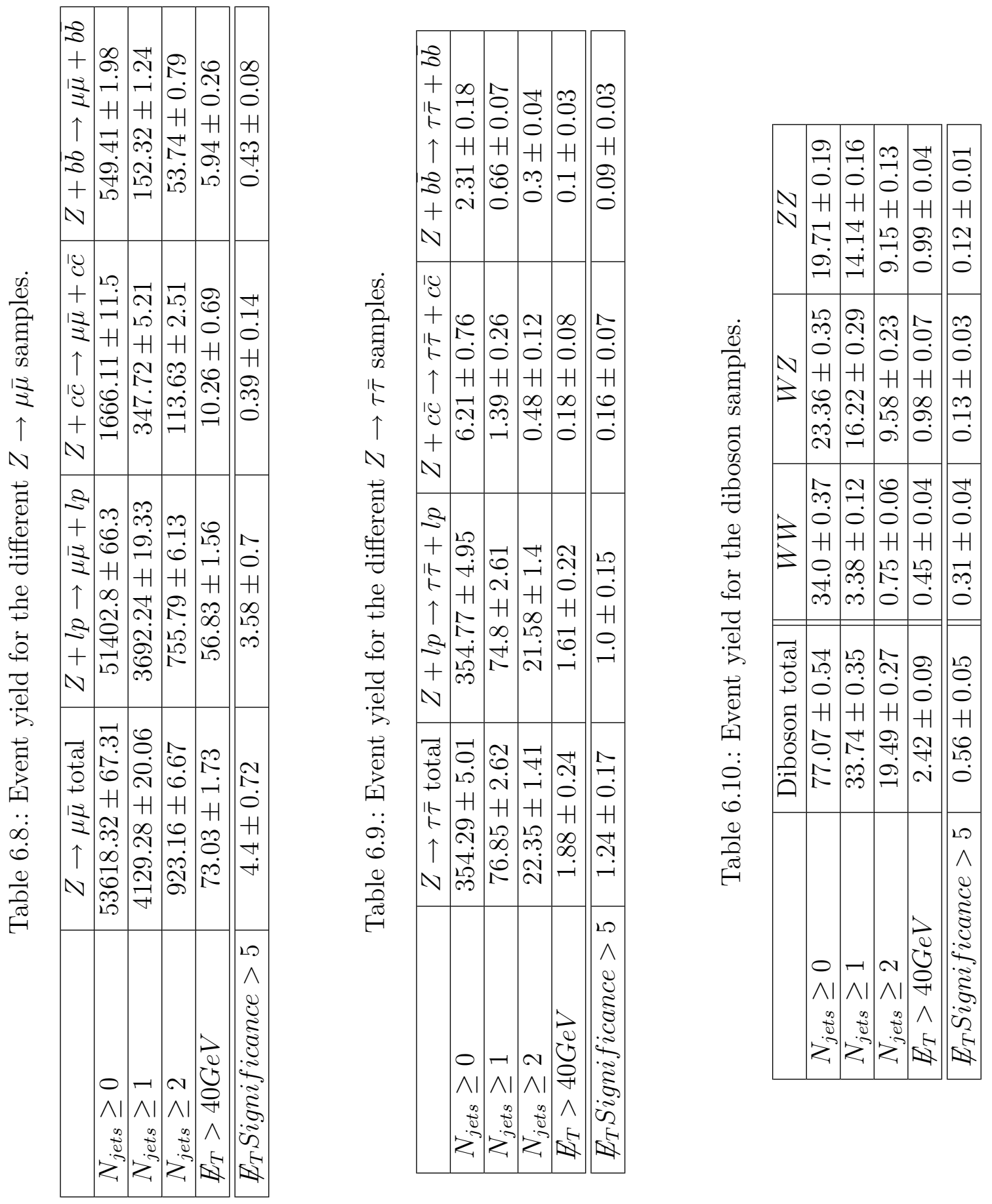
Table 6.11.: Selection efficiencies and related measured cross sections in $t \bar{t} \rightarrow \mu \bar{\mu}$ process for different top masses. The uncertainty on the selection efficiency are statistical only; The cross-section uncertainty shown includes both statistical and systematic ones. The cross-sections were extracted following the method in Sec. 6.5 .

\begin{tabular}{|c|c|c|}
\hline$m_{\text {top }}$ & $\epsilon_{t \bar{t}}$ & $\sigma(p \bar{p} \rightarrow t \bar{t})$ \\
\hline 160 & $6.1713 \pm 0.0754 \%$ & $5.6417_{-3.1379}^{+3.804}$ \\
\hline 165 & $6.4010 \pm 0.097 \%$ & $5.4393_{-3.0253}^{+3.6675}$ \\
\hline 170 & $6.8541 \pm 0.0795 \%$ & $5.0797_{-2.8253}^{+3.4251}$ \\
\hline 175 & $7.0701 \pm 0.0729 \%$ & $4.9245_{-2.739}^{+3.3205}$ \\
\hline 180 & $7.3914 \pm 0.0824 \%$ & $4.7105_{-2.62}^{+3.1761}$ \\
\hline 185 & $7.3783 \pm 0.1772 \%$ & $4.7188_{-2.6246}^{+3.1817}$ \\
\hline
\end{tabular}

\section{Vertex Cut and Opposite Muon Charge Cut Efficiency}

As the efficiency of the vertex selection is different in data and MC, we need to correct the $\mathrm{MC}$ efficiency to match data. The correction factor is the ratio of the cut efficiency in data and the $t \bar{t}$ Monte Carlo sample $\left(\epsilon_{\text {data }} / \epsilon_{M C}\right)$. The error on the correction factor is derived from the statistical error on the selection efficiencies.

A difference in selection efficiency is also observed for the opposite charge requirement $(O S)$ on the leading muons. Therefore, a correction factor is determined as the ratio of the OS efficiency in data and $t \bar{t}$ Monte Carlo. The vertex and OS correction factors as given in Tab. 6.12 are applied after the vertex and OS cuts, respectively.

Table 6.12.: Vertex and opposite muon charge selection efficiencies in data and $t \bar{t}$ MC. The quoted errors are statistical only.

\begin{tabular}{l|l|l|l} 
Vertex cut & Data efficiency & $t \bar{t}$ MC efficiency & Correction factor \\
\hline$|z|<60 \mathrm{~cm}$ & $98.5096 \pm 0.0039 \%$ & $99.3606 \pm 0.2779 \%$ & $0.9914 \pm 0.002773$ \\
$n_{\text {Track }} \geq 3$ & $98.4697 \pm 0.0039 \%$ & $99.8825 \pm 0.1194 \%$ & $0.9859 \pm 0.001179$ \\
$\Delta z(P V, \mu)$ & $97.1841 \pm 0.0053 \%$ & $99.7127 \pm 0.1866 \%$ & $0.9746 \pm 0.001825$ \\
\hline Total vertex correction & $94.2706 \pm 0.0074 \%$ & $98.9587 \pm 0.3534 \%$ & $0.9526 \pm 0.003403$ \\
\hline \hline Opposite charge cut & $99.9280 \pm 0.0009 \%$ & $99.6776 \pm 0.1946 \%$ & $1.00251 \pm 0.001957$ \\
\hline \hline Total & $94.2027 \pm 0.0074 \%$ & $98.6397 \pm 0.40155 \%$ & $0.9550 \pm 0.003887$
\end{tabular}




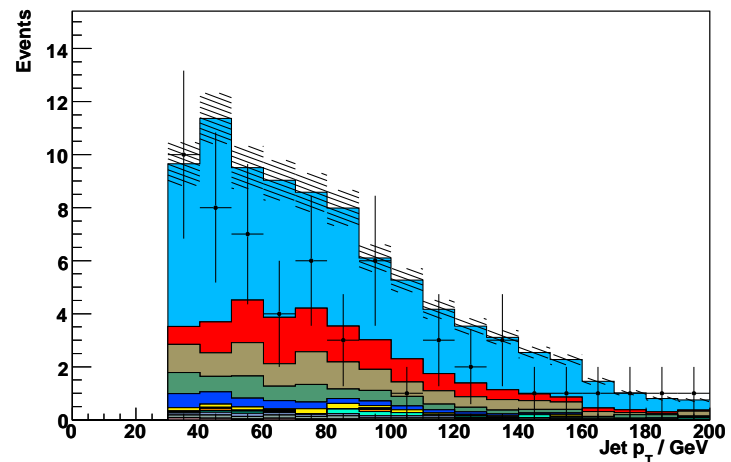

(a) After the $E_{T}$ cut

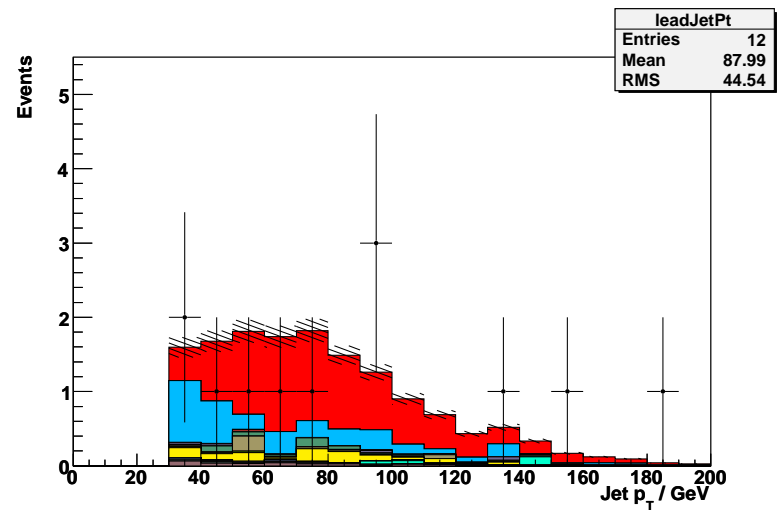

(b) Final selection

Figure 6.8.: Leading jet transverse momentum distribution.

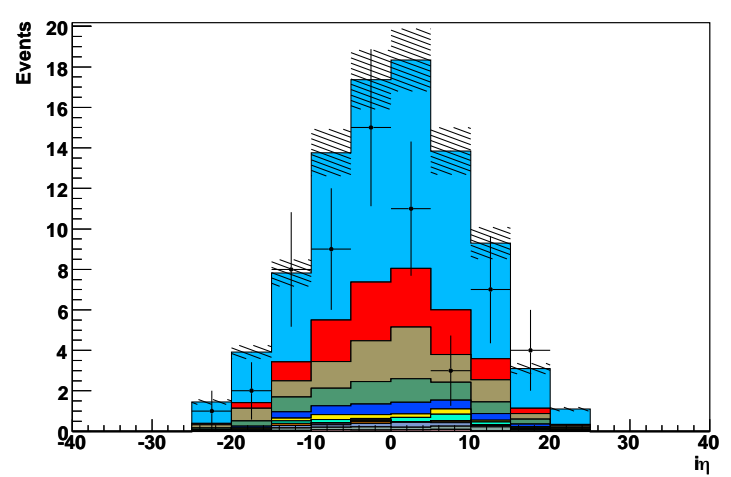

(a) After the $E_{T}$ cut

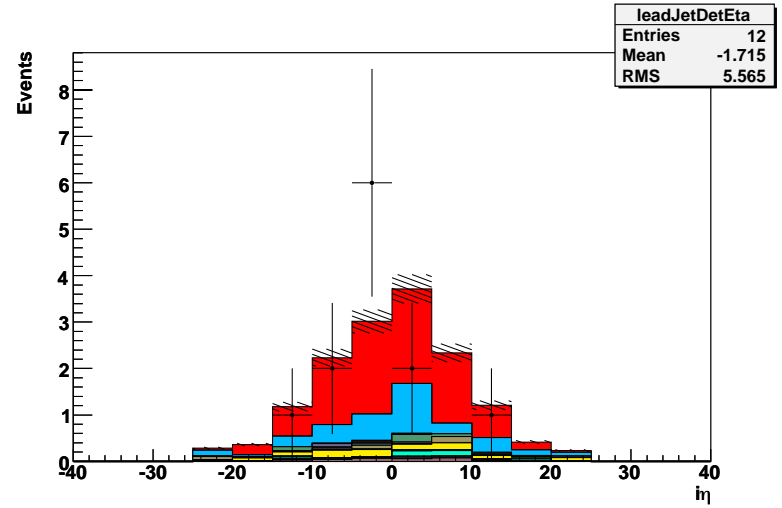

(b) Final selection

Figure 6.9.: Leading jet pseudo-rapidity distribution.

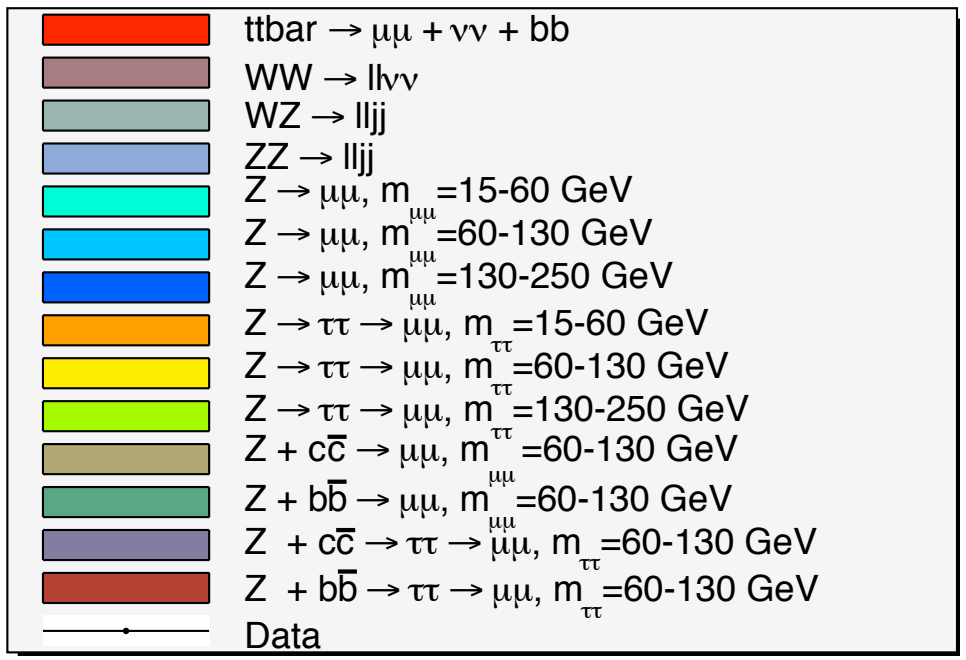

Figure 6.10.: Color code used in the plots shown on this page 


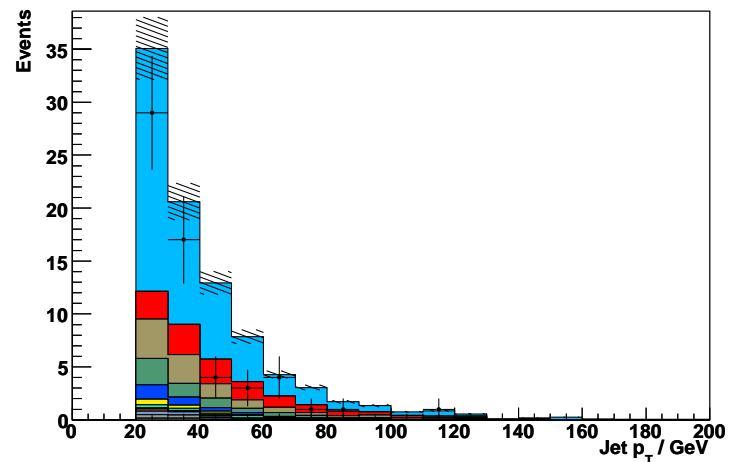

(a) After the $E_{T}$ cut

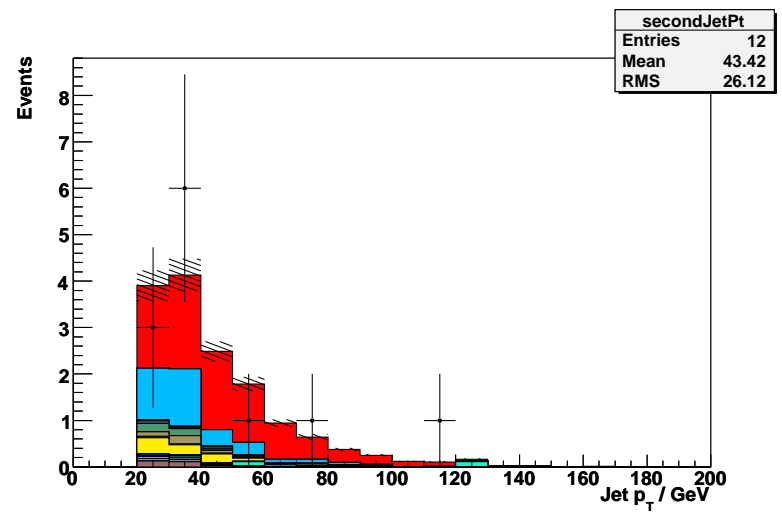

(b) Final selection

Figure 6.11.: Next-to-leading jet transverse momentum distribution.

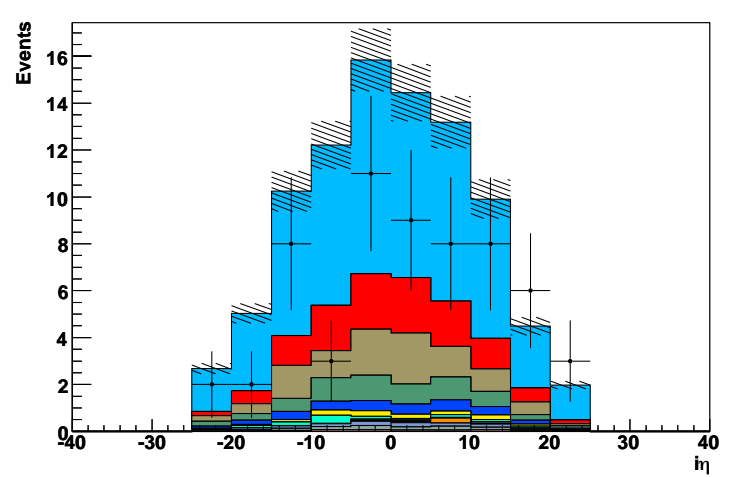

(a) After the $E_{T}$ cut

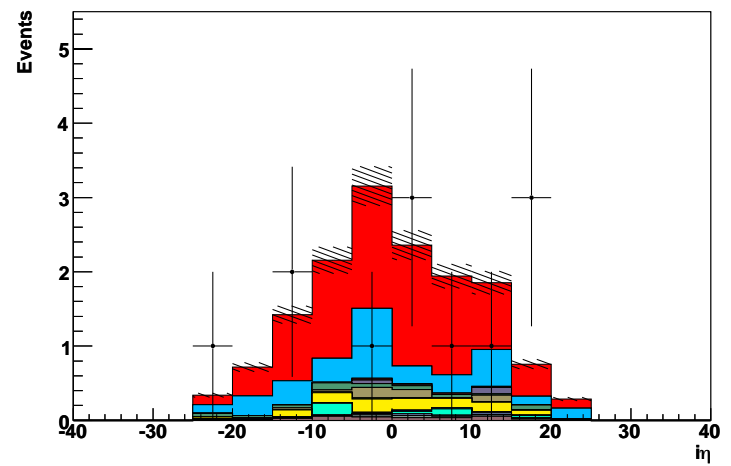

(b) Final selection

Figure 6.12.: Next-to-leading jet pseudo-rapidity distribution.

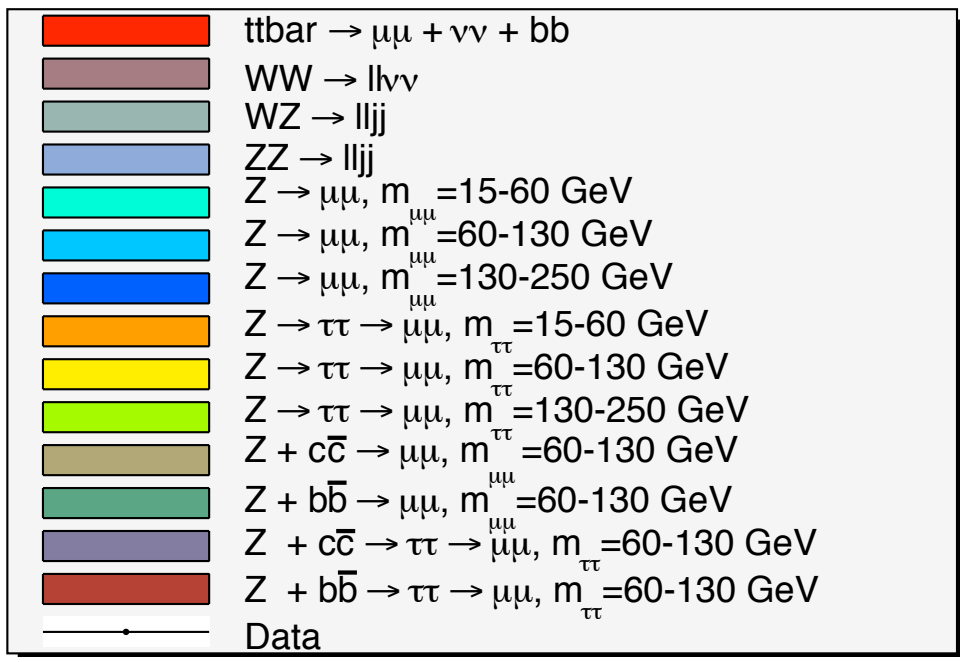

Figure 6.13.: Color code used in the plots shown on this page 


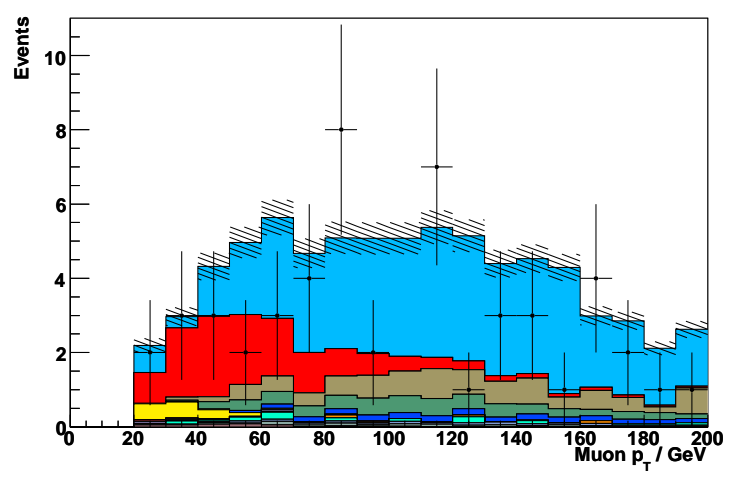

(a) After the $E_{T}$ cut

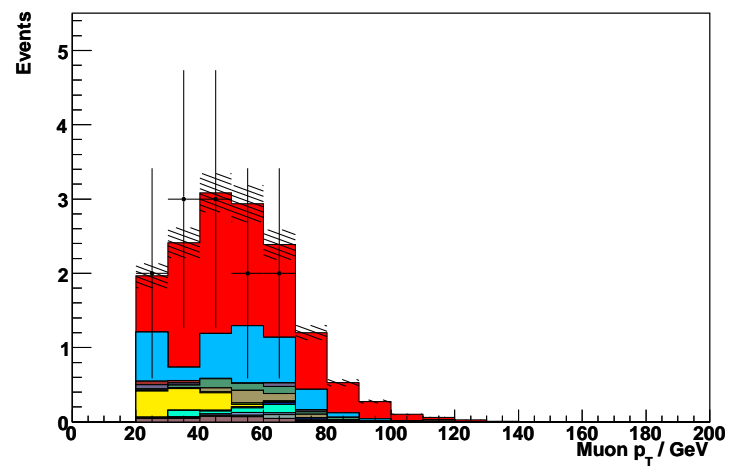

(b) Final selection

Figure 6.14.: Leading muon transverse momentum distributions.

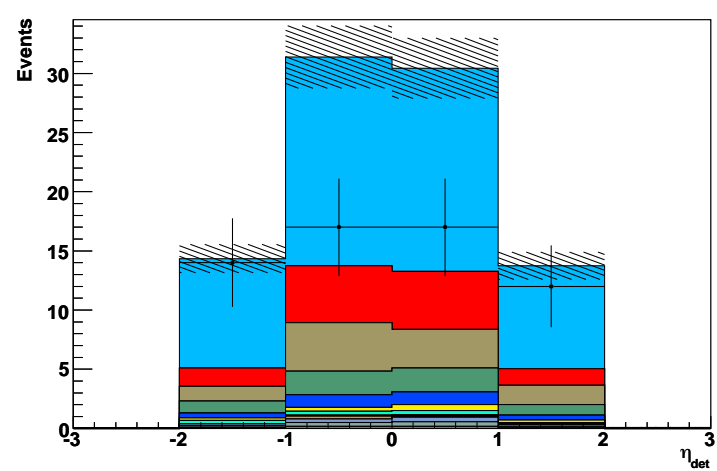

(a) After the $E_{T}$ cut

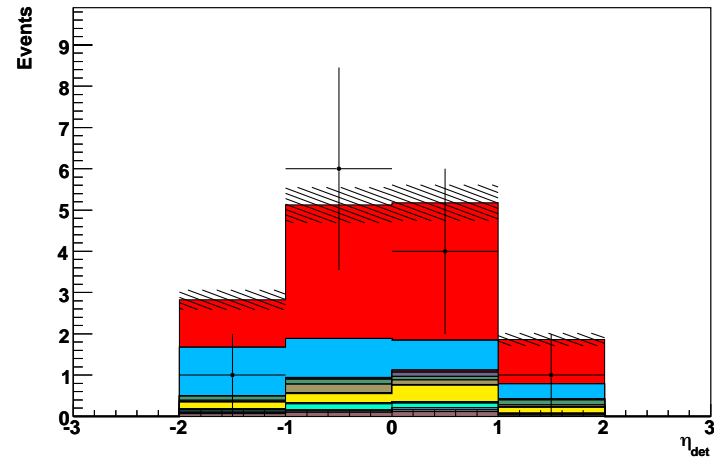

(b) Final selection

Figure 6.15.: Leading muon $\eta$ distributions.

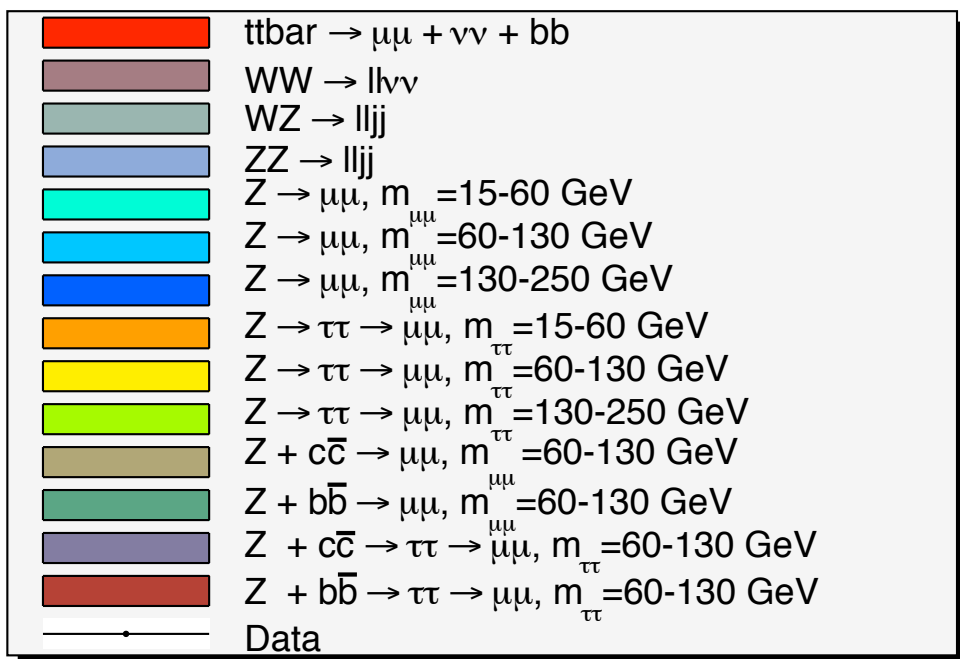

Figure 6.16.: Color code used in the plots shown on this page 


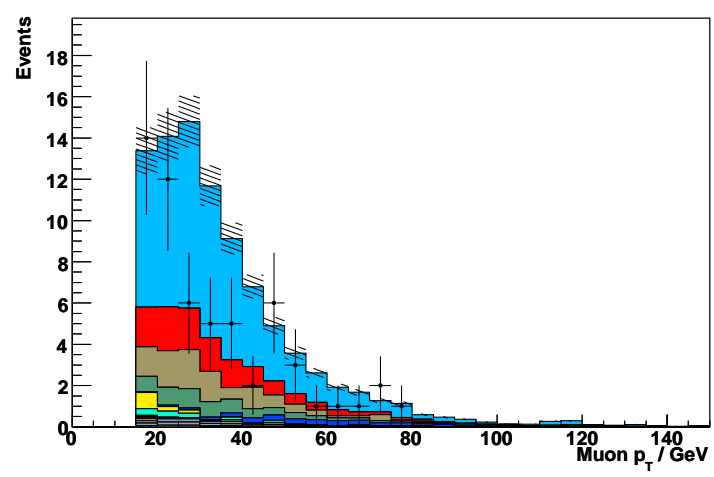

(a) After the $E_{T}$ cut

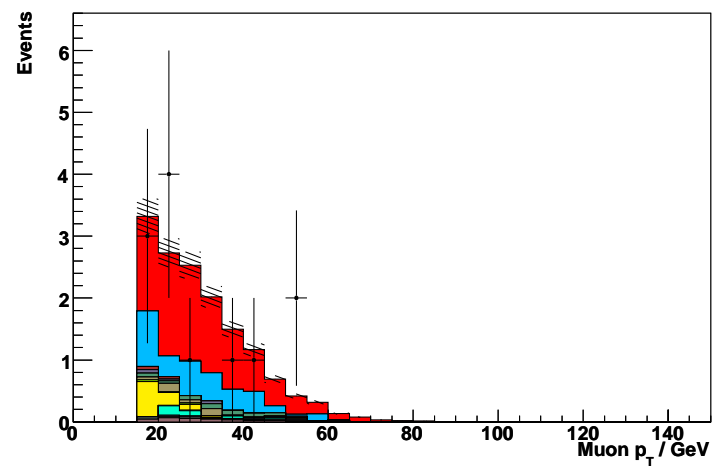

(b) Final selection

Figure 6.17.: Next-to-leading muon transverse momentum distributions.

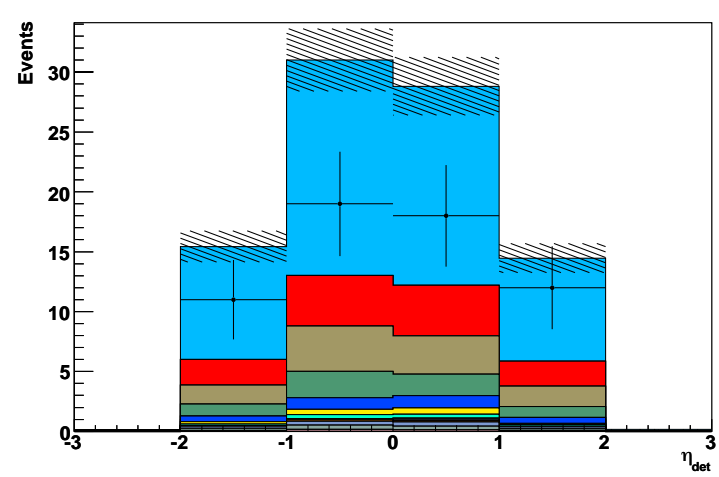

(a) After the $E_{T}$ cut

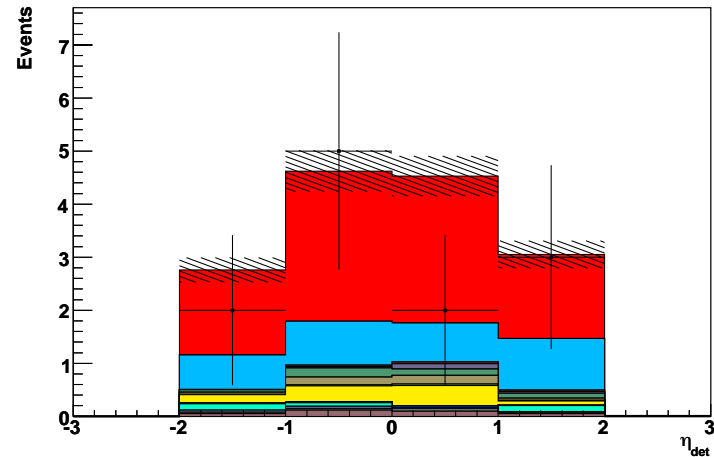

(b) Final selection

Figure 6.18.: Next-to-leading muon $\eta$ distributions.

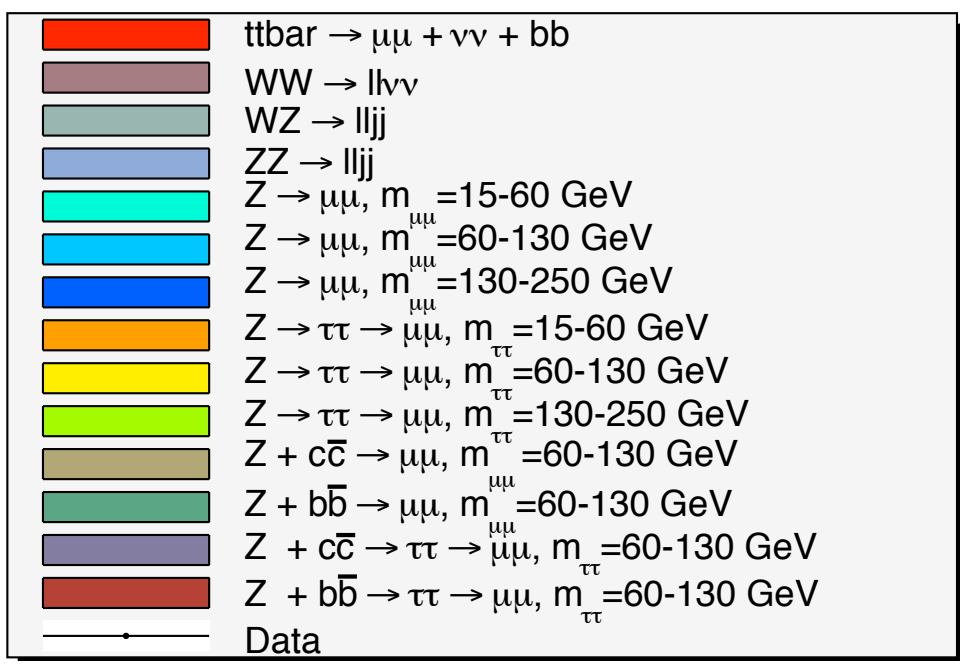

Figure 6.19.: Color code used in the plots shown on this page 


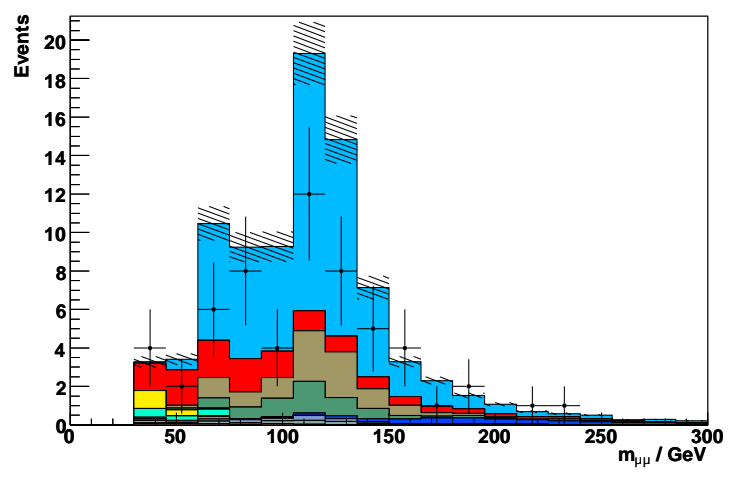

(a) After the $E_{T}$ cut

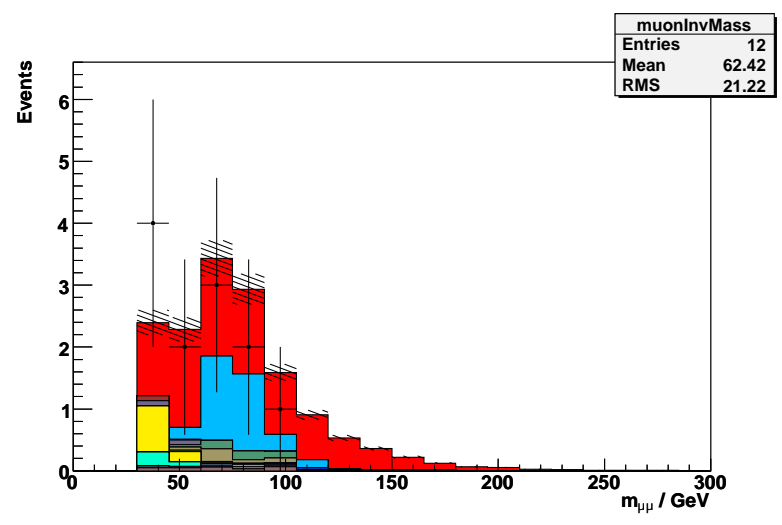

(b) Final selection

Figure 6.20.: Dimuon invariant mass distributions.

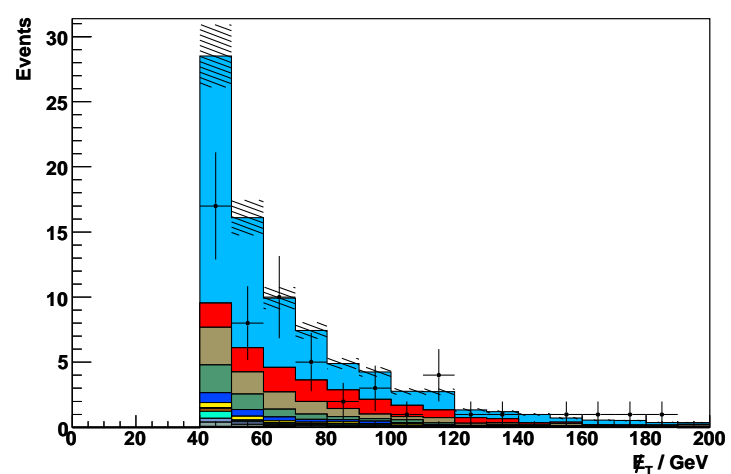

(a) After the $E_{T}$ cut

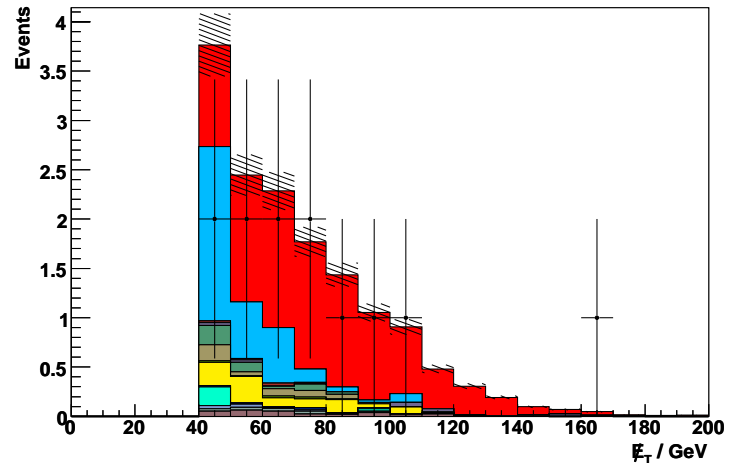

(b) Final selection

Figure 6.21.: $\mathbb{Z}_{T}$ distributions.

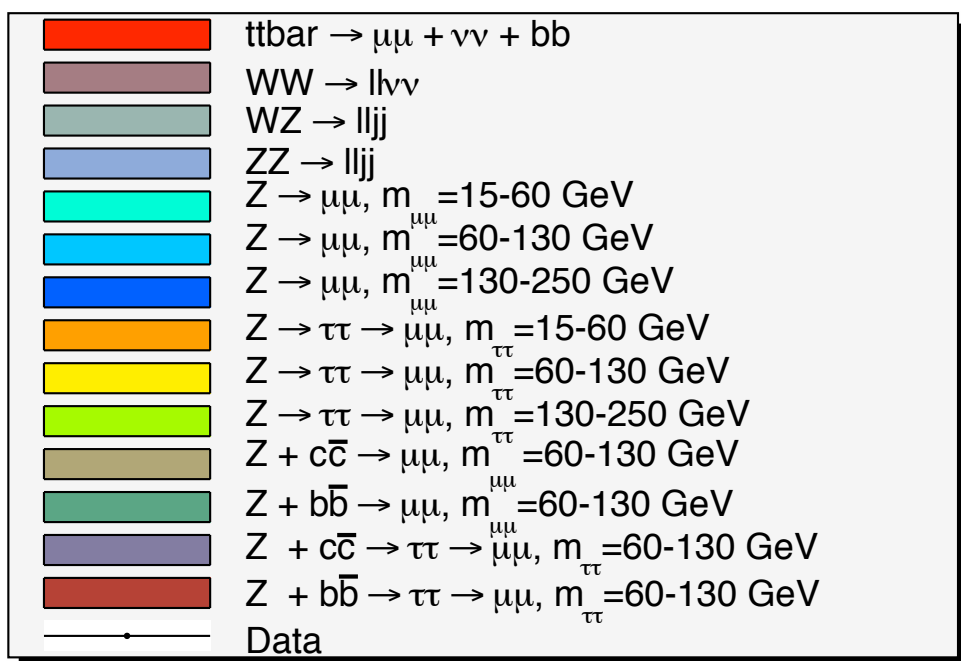

Figure 6.22.: Color code used in the plots shown on this page 


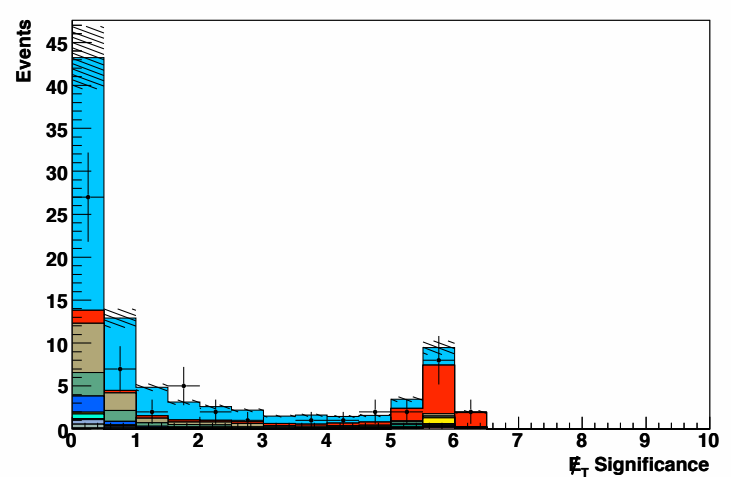

(a) After the $E_{T}$ cut

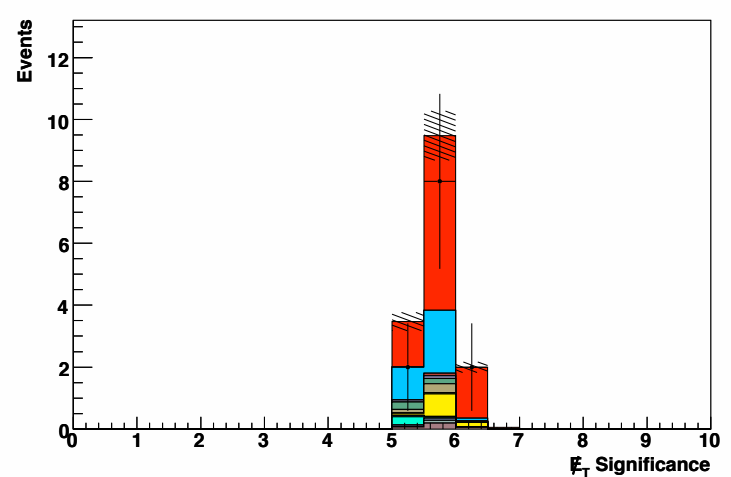

(b) Final selection

Figure 6.23.: $\mathbb{E}_{T}$ significance distributions.

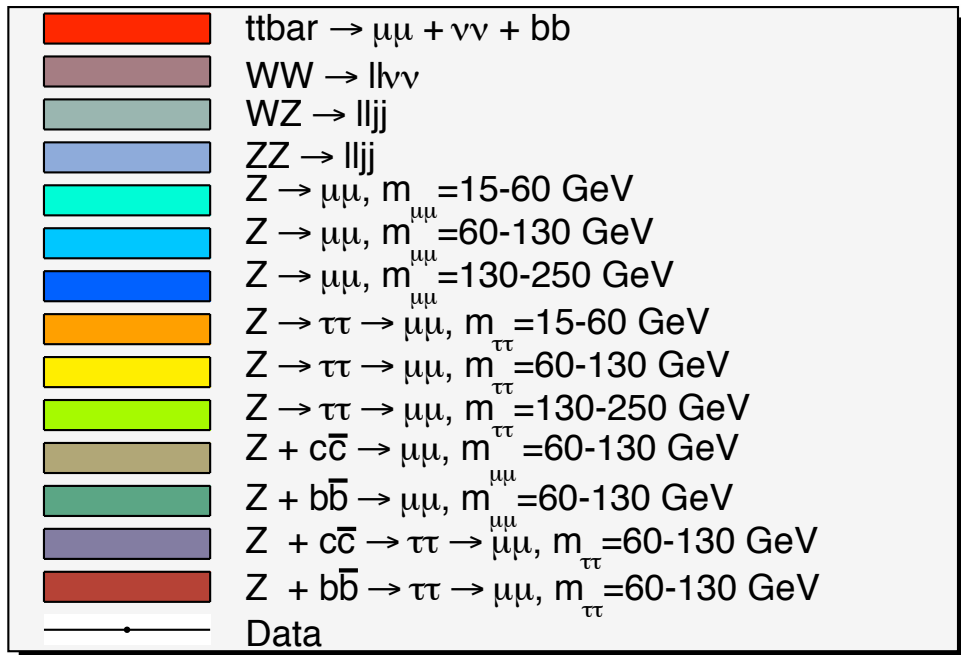

Figure 6.24.: Color code used in the plots shown on this page 


\subsubsection{Fake Muon Background Estimation}

Fake muon background corresponds to events with one isolated muon and one fake isolated muon, the latter coming mainly from heavy flavor quark decays. Such a signature corresponds for example to $W+$ jets events, where one of the jets is a b-jet. Because the fake muon isolation rate is low especially when requiring one isolated muon and two jets, this background is expected to be low. In order to estimate this background, we use a method similar to the one used in previous dimuon cross section analyses (see e.g. section VI E of reference [94]). The difference to the previous analyses lies in the $\Delta R($ jet, $\mu)<0.5$ cut used in the muon selection. All jets that pass the selection described in Sec. 6.3.1 are taken into account, i.e. all jets are required to have a transverse momentum greater than $20 \mathrm{GeV}$, as the cut on the leading jet $p_{T}$ is not imposed before the muon selection but at a later stage of the event selection. The number of "fake" muon background events is estimated via the so-called matrix method. This procedure requires two samples of events: a "tight" sample containing $N_{T}$ events passing all the $t \bar{t}$ selection cuts (1.15 in Sec. 6.3.5), and a "loose" sample $N_{L}$ where only one muon is isolated and fulfills the selection cut (8f), while the other muon needs not be isolated and thus need not fulfill the selection cut (8f). The number of events in each sample are related to the signal and background event yields by the relations:

$$
\begin{array}{r}
N_{L}=N^{Z+t o p}+N^{W+Q C D} \\
N_{T}=\epsilon_{\text {sig }} N^{Z+t o p}+f_{\mu} N^{W+Q C D}
\end{array}
$$

where $N^{Z+t o p}$ is the combination of $Z / \gamma^{*}$ and $t \bar{t} \rightarrow \mu \bar{\mu}$ events, and $\epsilon_{\text {sig }}$ and $f_{\mu}$ are the muon isolation probabilities for the signal-like and background muons respectively. The signal efficiency $\epsilon_{\text {sig }}$ and the muon fake rate $f_{\mu}$ are determined from the di-muon data sample (Sec. 5). To determine $\epsilon_{s i g}$ we define a tag muon, being the isolated next-to-leading $p_{T}$ muon and a probe muon, being the leading $p_{T}$ muon without any isolation criteria. $\epsilon_{s i g}$ is then defined as $\epsilon_{\text {sig }}=N_{\text {isolated }}^{\text {probe }} / N_{\text {isolated }}^{\text {tag }}$. To determine the muon fake rate, we define a tag muon, being the non-isolated leading $p_{T}$ muon, and a probe muon, being the next-to-leading $p_{T}$ muon without any isolation criteria. $f_{\mu}$ is then defined as $f_{\mu}=N_{\text {isolated }}^{\text {probe }} / N_{\text {nonisolated }}^{\text {tag }}$.

Solving Eqns. 6.5 and 6.6 for $N^{W+Q C D}$ yields

$$
N^{W+Q C D}=N_{L} \frac{\frac{N_{T}}{N_{L}}-\epsilon_{s i g}}{f_{\mu}-\epsilon_{s i g}} .
$$

The fake rate and the isolation efficiency are measured in each jet multiplicity bin, and shown in Tab. 6.13.

After all cuts, we find 12 events in the tight and 19 events in the loose sample, which gives a background yield for isolation fakes of

$$
N_{\text {fake }}=0.158 \pm 0.162 .
$$


Table 6.13.: The measured fake rates and signal efficiencies. All errors are statistical ones.

\begin{tabular}{c|c|c} 
Jet Multiplicity & Fake rate $f_{\mu}$ & Signal efficiency $\epsilon_{\text {sig }}$ \\
\hline$\geq 0$ & $0.1410 \pm 0.00921$ & $0.9714 \pm 0.00072$ \\
$\geq 1$ & $0.1410 \pm 0.00921$ & $0.7739 \pm 0.00598$ \\
$\geq 2$ & $0.0516 \pm 0.00642$ & $0.7434 \pm 0.01215$
\end{tabular}

\subsection{Systematic Errors}

Various sources of systematic uncertainties were studied to investigate their influence on signal efficiencies and background expectations. The most important contributions come from the uncertainty on the jet energy scale, the uncertainties in the Monte Carlo reweightings applied and the efficiency corrections in Monte Carlo. Other contributions arise from the calculation of the expected fake muon background, the dependency of the signal selection efficiency on the top mass, the ratio of the LO to (N)NLO cross sections of the $\mathrm{Z}$ and diboson background processes and the measurement of the integrated luminosity.

- JES. The jet energies and transverse momenta in MC were modified by $\pm 1 \sigma_{J E S}$ derived together with the JSSR corrections. This new correction is propagated to the missing $E_{T}$.

- Jet Resolution. The JSSR procedure applies additional smearing to the MC jets, in order to account for the different jet $p_{T}$ resolution in data and MC. The parameters of the jet energy smearing are varied by the size of the uncertainty on the jet energy resolution parameters in MC. To compute the final cross-section systematic error, this systematics is treated as $100 \%$ correlated for all MC samples.

- Jet ID. The standard procedure defined by the jet ID group has been used which consists of varying the jet ID efficiency by its uncertainty.

- Trigger systematics. The cross-section systematic error has been evaluated by varying the trigger efficiency by its statistical uncertainty.

- Muon ID and track scale factor systematics. The systematic errors on the $\mathrm{MC} /$ Data correction factor are estimated in the muon certification note [59]. We use the systematic errors computed for loose muons, $0.4 \%$, and medium offline track match, $0.7 \%$.

- Muon isolation scale factor systematics. This systematic uncertainty is not estimated by the muon ID group. We are using the systematic error estimated by the single top group [95]. The uncertainty was estimated from a distribution showing the dependence of the isolation scale factor versus the number of jets. The resulting uncertainty is $2 \%$. This uncertainty covers the dependences (versus $\eta$ and $p_{T}$ ) thatare not taken into account, but are available in the muon certification note [59].

- Primary vertex and opposite sign charge selection systematics. The selection efficiency for the vertex selection and opposite charged muon pairs is found to be different in data and MC (see Section 6.3.5, table 6.12). Taking into account the 
statistical error on the scale factors, we assign a $0.34 \%$ systematic uncertainty for PV ID and a $0.2 \%$ uncertainty for the opposite charge requirenment.

- Uncertainty in modeling $\mathbb{E}_{T}$ in signal and background Monte Carlo. The $E_{T}$ distribution in the data sample and of the simulated signal and background samples can only be brought into agreement by not propagating the jet smearing to the missing transverse energy. This is observed in the dielectron final state as well as the dimuon final state and other analyses.

However, as we cut on $E_{T}$ in this analysis it is important to estimate the difference between data and our simulation. This difference will be taken into account as a systematic uncertainty.

As part of the $E_{T}$ modeling uncertainty is already covered by the varying of the jet energy scale, ID and resolution in addition to the muon object ID, we will estimate the uncertainty on the modeling of the fake $E_{T}$ with the following method. The missing transverse energy distributions in data and the sum of all MC samples are first normalized to unity. We then compare the integral of the two distributions between 0 and $40 \mathrm{GeV}$. The ratio between data and MC is taken as the uncertainty on the $E_{T}$ modeling.

- Signal modeling. To estimate the uncertainty on the signal modeling, we compare the signal selection efficiencies measured on ALPGEN+PYTHIA Monte Carlo samples with the selection efficiency measured on PYTHIA MC.

- Data quality flag systematics. The systematic uncertainty on the measured data quality flag efficiency was estimated to be $0.5 \%$ [93].

- Fake muon background. The statistical uncertainty on the fake muon background is taken into account as a systematic uncertainty.

- Signal branching ratio, diboson and $Z$ Monte Carlo systematics. Apart from the statistical uncertainties on the background estimation, there are a number of systematic uncertainties that have to be included for all or a subset of the background MC samples.

The error on the $t \rightarrow b l \nu$ branching ratio is calculated using the measured $W \rightarrow l \nu_{l}$ and $\tau \rightarrow \mu \nu_{\mu} \nu_{\tau}$ branching ratios [18]. For the $Z+\mathrm{HF}$ and diboson samples, we assign a $20 \%$ error on the $\mathrm{K}$-factor (including the $\mathrm{HF}$ scale factor in the case of $Z+\mathrm{HF}$ ). The error on the $Z+$ lp cross-section is taken from D $\varnothing$ Note 5268 [86].

- Systematics due to Data-Monte Carlo luminosity profile difference. The DØ simulation uses overlaid zero bias events to reproduce detector and luminosity effects from real data. This requires an exact matching of the overlaid zero bias luminosity profile to the luminosity profile in the data sample used in this analysis. Unfortunately the set of zero bias events used in MC does not correspond exactly to the one collected in the Run IIa (MC events generation started before Run IIa data taking has been finished). In order to reduce the impact of this discrepancy on the selection, the luminosity reweighting procedure has been used in MC. This procedure increases the weights of $\mathrm{MC}$ events with high luminosity. Unfortunately, the exact match of luminosity profile requires very high weights for MC events with 
high luminosity. In order to limit the statistical fluctuation because of the high individual weight, the maximal allowed weight in the luminosity reweighting procedure has been set to 5 . In order to estimate the systematics due to the imperfection of the matching procedure all MC samples have been reweighed without any maximal limit on the weight and the difference in the efficiency with the default case (maximal weight equals to 5) has been assigned as systematics.

- Z vertex distribution difference between data \& Monte Carlo. The z vertex distribution simulated in $\mathrm{MC}$ events is quite different from the data. In order to correct for this difference the corresponding reweighting from the caf_mc_util package has been applied. In order to estimate the systematics for this correction, an alternative parametrization for the beam shape could be used. The difference in the event selection efficiency between default and alternative parametrization as derived by the $t \bar{t} \rightarrow e \mu$ analysis [96] is quoted as a systematics uncertainty.

- $Z p_{T}$ reweighting systematics. The difference in $Z \rightarrow \mu \bar{\mu}$ and $Z \rightarrow \tau \bar{\tau}$ event yield between the default and $\pm 1 \sigma$ shifted weight is assigned to this systematic uncertainty.

- Systematics due to PDF uncertainties. The standard caf_pdfreweight package has been used for this estimation. The systematic uncertainty due to the choice of PDF is estimated by varying the PDF using the method in Ref. [22], Sec. 2.3

- B jet energy scale \& jet shifting. To estimate the uncertainty due to JES sample dependence, the SSR jet shifting was turned off in the signal sample. In addition, the B jet scale was varied by $0.92 \%$. These systematic errors account for the JES sample dependence.

- Uncertainty associated with the top mass. The uncertainty due to the effect of the top mass on the full selection efficiency of Table 6.6 is estimated using Monte Carlo Samples generated with different top masses. We evaluate the relative differences in efficiencies varying the top mass by $10 \mathrm{GeV}$ down and $15 \mathrm{GeV}$ up from the $170 \mathrm{GeV}$ reference top mass (used in Table 6.6). Following the general top group strategy, we will not add this systematics to all other sources, but it will be cited separately. Fitting the cross-sections listed in Table 6.11 with a linear function and comparing with the cross section-mass dependence in [45] and [42] leads to the plot shown in Fig. 6.25. The fit function is given by

$$
\sigma_{t \bar{t}}=11.97 \mathrm{pb}-m_{\text {top }} \times 0.0389 \mathrm{pb} / \mathrm{GeV}
$$

- Monte Carlo statistics. The statistic uncertainty on the signal selection efficiency and background yields is taken into account as a systematic uncertainty. For the diboson MC, the uncertainty we assign to the NLO cross-section is comparable to the error due to the limited MC statistics. For the $Z \mathrm{MC}$, the statistical error is dominating. However, we already made full use of the available HF skimmed Z Monte Carlo samples. Improving significantly on the MC statistics would mean doubling our $Z$ MC samples, which seems not justified given the analysis is still limited by (data) statistics.

Tab. 6.15 recapitulates all the systematic uncertainties taken into account. The uncorrelated errors correspond to the statistical uncertainties on the MC samples and the fake muon background. 


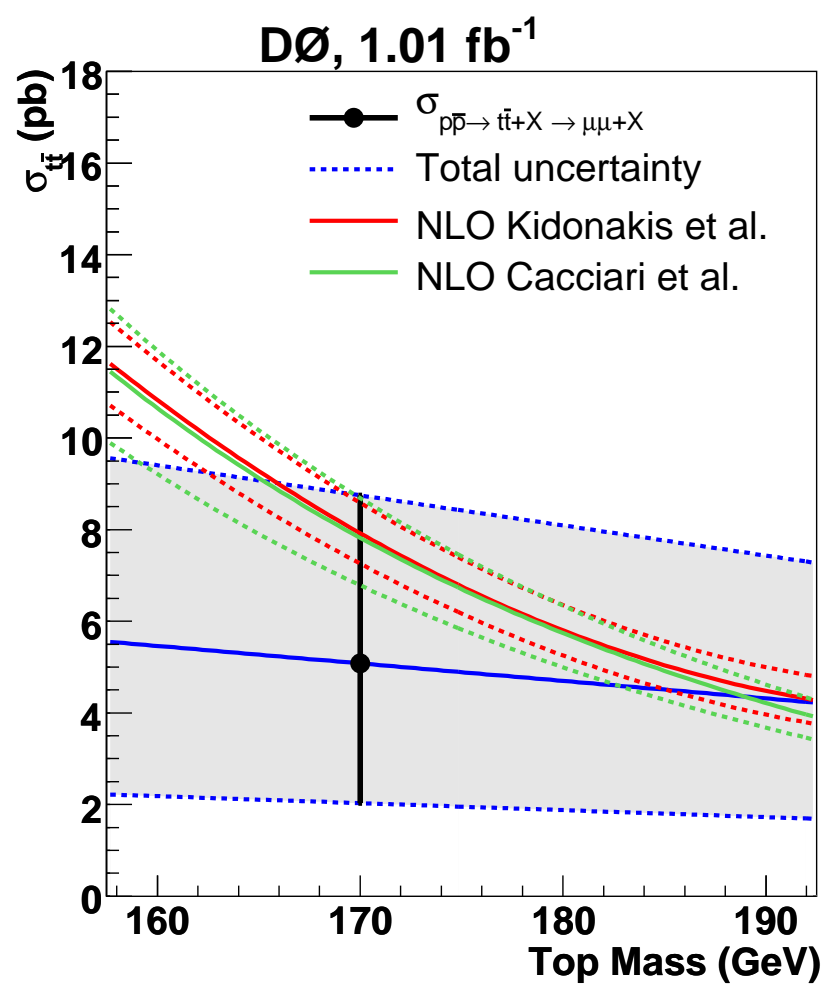

Figure 6.25.: Dependence of the top quark pair production cross section on the top mass. 


\begin{tabular}{|c|c|c|c|c|c|c|c|c|c|c|c|c|c|c|c|c|c|c|c|c|c|c|c|c|c|}
\hline & لٌْ & 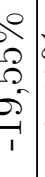 & $\begin{array}{l}0 \\
0 \\
0 \\
2 \\
0 \\
0 \\
0\end{array}$ & $\begin{array}{l}80 \\
0 \\
10 \\
0 \\
0 \\
1\end{array}$ & $\mid \begin{array}{l}0 \\
\grave{D} \\
\infty \\
0 \\
0\end{array}$ & $\begin{array}{l}\qquad 0 \\
0 \\
0 \\
0 \\
0 \\
1\end{array}$ & 各 & 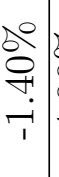 & & 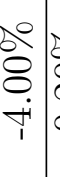 & 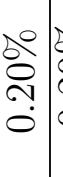 & 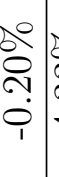 & 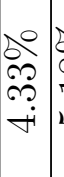 & 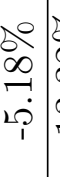 & 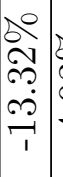 & \begin{tabular}{l|l}
0 & \\
0 & \\
0 & \\
+ & 1
\end{tabular} & & 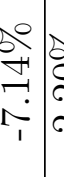 & & 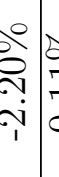 & & & & & \\
\hline & B & 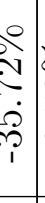 & $\begin{array}{l}50 \\
0 \\
0 \\
0 \\
0 \\
0\end{array}$ & $\begin{array}{l}\Delta 0 \\
0 \\
0 \\
10 \\
0 \\
1\end{array}$ & $\mid \begin{array}{l}0 \\
0 \\
\infty \\
0 \\
0\end{array}$ & $\begin{array}{l}\Delta 0 \\
0 \\
0 \\
0 \\
0 \\
1\end{array}$ & $\begin{array}{l}\stackrel{0}{0} \\
\stackrel{2}{+} \\
\stackrel{+}{-1}\end{array}$ & 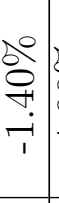 & \begin{tabular}{c|c}
$\stackrel{0}{a}$ \\
$\stackrel{2}{8}$ \\
$\stackrel{+}{+}$
\end{tabular} & $\begin{array}{c}\Delta \\
8 \\
8 \\
\dot{+} \\
\dot{+}\end{array}$ & 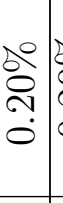 & $\begin{array}{l}00 \\
0 \\
0 \\
\vdots \\
0 \\
1\end{array}$ & $\begin{array}{l}\infty \\
\infty \\
\infty \\
\dot{+} \\
\dot{+}\end{array}$ & 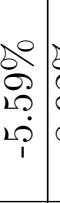 & 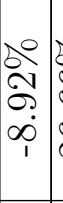 & 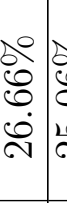 & & 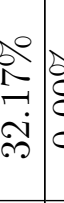 & & 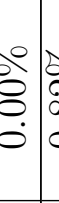 & & 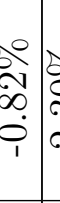 & & 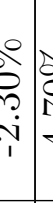 & \\
\hline & 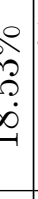 & 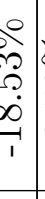 & $\begin{array}{l}0 \\
0 \\
0 \\
0 \\
0 \\
0 \\
0\end{array}$ & $\begin{array}{l}00 \\
0 \\
0 \\
0 \\
0 \\
1\end{array}$ & $\begin{array}{l}0 \\
0 \\
\infty \\
\infty \\
0 \\
\dot{0}\end{array}$ & $\begin{array}{l}00 \\
0 \\
0 \\
0 \\
0 \\
1\end{array}$ & & $\mid \begin{array}{c}0 \\
0 \\
0 \\
⿱ \\
1 \\
1\end{array}$ & $\mid \begin{array}{c}0 \\
8 \\
8 \\
\dot{\rightarrow}\end{array}$ & $\begin{array}{c}80 \\
8 \\
8 \\
+ \\
\dot{1}\end{array}$ & $\begin{array}{l}\stackrel{0}{o} \\
\stackrel{1}{2} \\
\vdots \\
0\end{array}$ & 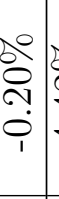 & 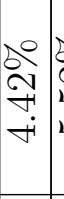 & 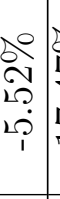 & 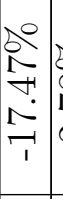 & \begin{tabular}{l|l}
$c$ & 0 \\
0 & 0 \\
1 & \\
$i$ & 1
\end{tabular} & 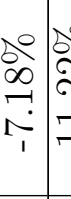 & 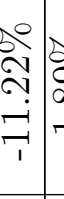 & & 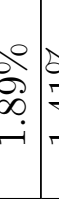 & & 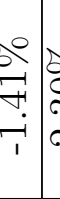 & & & \\
\hline & $\begin{array}{l}8 \\
\\
\\
\end{array}$ & 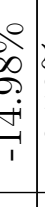 & $\begin{array}{l}0 \\
0 \\
0 \\
2 \\
0 \\
0 \\
0\end{array}$ & $\begin{array}{l}\Delta 0 \\
0 \\
0 \\
0 \\
0 \\
0 \\
1\end{array}$ & $\begin{array}{l}0 \\
0 \\
\infty \\
\infty \\
0 \\
0\end{array}$ & $\begin{array}{l}\Delta 0 \\
0 \\
\infty \\
0 \\
0 \\
1\end{array}$ & 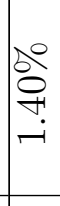 & 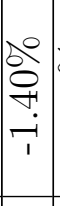 & $\mid \begin{array}{l}0 \\
0 \\
8 \\
0 \\
\dot{+}\end{array}$ & $\begin{array}{c}80 \\
8 \\
8 \\
+ \\
\dot{1}\end{array}$ & 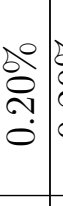 & 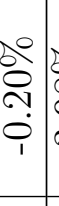 & $\begin{array}{l}\infty \\
0 \\
\infty \\
\infty \\
\infty \\
\infty\end{array}$ & 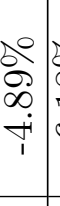 & \begin{tabular}{c|c}
0 & 0 \\
$\infty$ & \\
-1 & 1 \\
0 & 1 \\
1 & 1
\end{tabular} & 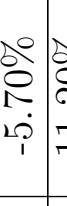 & 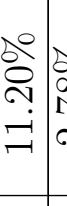 & 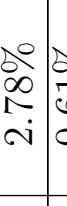 & & $\begin{array}{l}0 \\
0 \\
0 \\
0 \\
0 \\
1 \\
1\end{array}$ & & 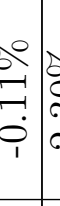 & & $\begin{array}{c}0 \\
\vdots \\
\vdots \\
i \\
i\end{array}$ & \\
\hline & 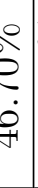 & $\begin{array}{l}0 \\
e \\
0 \\
0 \\
i \\
1\end{array}$ & $\begin{array}{l}50 \\
0 \\
0 \\
0 \\
0 \\
0\end{array}$ & $\begin{array}{l}\Delta 0 \\
0 \\
0 \\
10 \\
0 \\
1\end{array}$ & $\begin{array}{l}00 \\
0 \\
0 \\
0 \\
0 \\
0\end{array}$ & $\begin{array}{l}\Delta 0 \\
0 \\
\infty \\
0 \\
0 \\
1\end{array}$ & 各 & 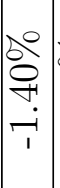 & 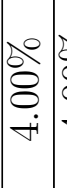 & 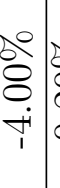 & 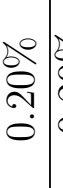 & 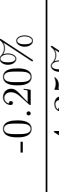 & 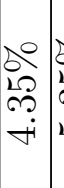 & 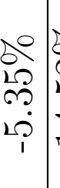 & 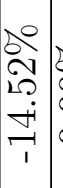 & $\begin{array}{lll} & \\
0 & \\
8 & \\
0 & \\
0 & \\
0 & \end{array}$ & 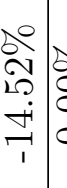 & \begin{tabular}{l|l}
0 \\
8 \\
8 \\
$\vdots$ \\
0
\end{tabular} & & \begin{tabular}{l|l}
0 \\
8 \\
8 \\
$\dot{0}$
\end{tabular} & & $\begin{array}{ll}0 & 0 \\
0 & 0 \\
0 \\
0 \\
i \\
1\end{array}$ & & 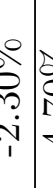 & 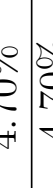 \\
\hline & $\begin{array}{l}0 \\
0 \\
0 \\
0 \\
v\end{array}$ & $\begin{array}{l}0 \\
0 \\
0 \\
0 \\
-1 \\
-1\end{array}$ & $\begin{array}{l}0 \\
0 \\
0 \\
0 \\
0 \\
0\end{array}$ & $\begin{array}{l}\qquad 0 \\
0 \\
10 \\
0 \\
0 \\
1\end{array}$ & $\begin{array}{l}0 \\
\stackrel{0}{0} \\
\infty \\
0 \\
0\end{array}$ & $\begin{array}{l}00 \\
0 \\
\infty \\
0 \\
0 \\
1\end{array}$ & $\begin{array}{l}\stackrel{0}{0} \\
\text { Oे } \\
\stackrel{+}{-} \\
-1\end{array}$ & 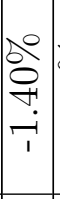 & $\left|\begin{array}{c}0 \\
\hdashline \\
0 \\
0 \\
\dot{+}\end{array}\right|$ & $\begin{array}{c}0 \\
8 \\
8 \\
\dot{+} \\
\dot{+}\end{array}$ & 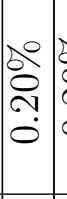 & $\begin{array}{ll}0 & 0 \\
0 & 1 \\
0 & 1 \\
0 & 1\end{array}$ & 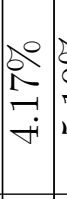 & 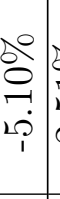 & 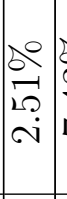 & 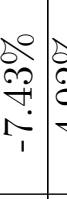 & 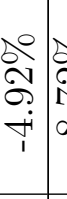 & 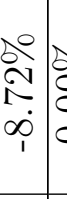 & & $\begin{array}{lll}0 & 0 \\
8 & 0 \\
0 & 0\end{array}$ & & 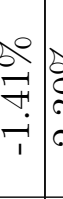 & & & \\
\hline & t. & 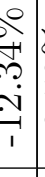 & $\begin{array}{l}\text { de } \\
0 \\
\stackrel{0}{10} \\
0\end{array}$ & $\begin{array}{l}20 \\
0 \\
0 \\
0 \\
0 \\
0 \\
1\end{array}$ & $\begin{array}{l}\infty \\
\stackrel{0}{0} \\
\infty \\
\infty \\
0\end{array}$ & $\begin{array}{l}00 \\
0 \\
0 \\
0 \\
0 \\
1\end{array}$ & 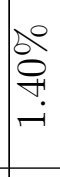 & $\mid \begin{array}{c}0 \\
0 \\
O \\
-1 \\
i\end{array}$ & $\mid$\begin{tabular}{c}
0 \\
\hdashline \\
8 \\
0 \\
$\dot{\forall}$
\end{tabular} & $\begin{array}{c}0 \\
8 \\
8 \\
\dot{1} \\
1\end{array}$ & $\left|\begin{array}{c}0 \\
0 \\
0 \\
⿱ \\
0 \\
0\end{array}\right|$ & 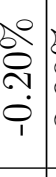 & $\mid \begin{array}{c}0 \\
0 \\
8 \\
0 \\
n\end{array}$ & $\begin{array}{c}0 \\
0 \\
\infty \\
\infty \\
+ \\
+\end{array}$ & 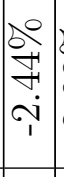 & 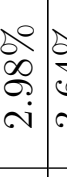 & 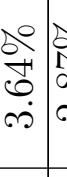 & $\begin{array}{ll}0 & 0 \\
0 & \\
\infty & 0 \\
i & \\
i & 0\end{array}$ & & 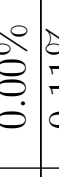 & & 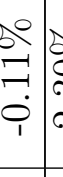 & & & \\
\hline & 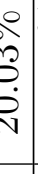 & 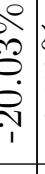 & $\begin{array}{l}0 \\
0 \\
0 \\
0 \\
0\end{array}$ & $\begin{array}{l}0 \\
0 \\
0 \\
0 \\
0 \\
0 \\
1\end{array}$ & 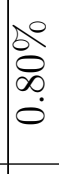 & $\begin{array}{l}0 \\
0 \\
\infty \\
0 \\
0 \\
1\end{array}$ & 용 & 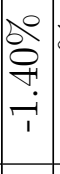 & $\mid$\begin{tabular}{c}
0 \\
$\stackrel{0}{0}$ \\
8 \\
\hdashline \\
$\dot{\forall}$
\end{tabular} & $\begin{array}{c}80 \\
8 \\
8 \\
\dot{+}+1\end{array}$ & $\begin{array}{c}0 \\
\stackrel{0}{0} \\
\stackrel{2}{N} \\
0\end{array}$ & 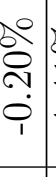 & $\begin{array}{l}\stackrel{0}{\circ} \\
\vec{F} \\
\dot{+}\end{array}$ & 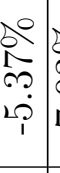 & $\begin{array}{c}0 \\
0 \\
0 \\
0 \\
1\end{array}$ & \begin{tabular}{l|l}
0 & 0 \\
0 & 0 \\
0 & 0 \\
0 & 0 \\
0 & 0 \\
1 & 0
\end{tabular} & 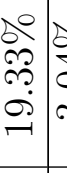 & 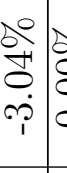 & \begin{tabular}{l|l}
0 & 8 \\
8 & 8 \\
0 & 0
\end{tabular} & \begin{tabular}{l|l}
0 \\
8 \\
8 \\
0
\end{tabular} & & $\begin{array}{l}0 \\
0 \\
\overrightarrow{1} \\
\dot{0} \\
1\end{array}$ & 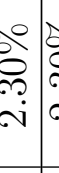 & $\begin{array}{l}0 \\
0 \\
0 \\
\vdots \\
i \\
i\end{array}$ & \\
\hline & טָo & 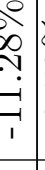 & $\begin{array}{l}0 \\
0 \\
0 \\
0.0 \\
0\end{array}$ & $\begin{array}{l}0 \\
0 \\
0 \\
0 \\
0 \\
0 \\
1\end{array}$ & $\begin{array}{l}0 \\
0 \\
\infty \\
0 \\
0\end{array}$ & $\begin{array}{l}00 \\
0 \\
0 \\
0 \\
0\end{array}$ & 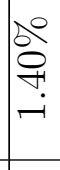 & 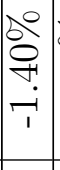 & $\begin{array}{l}0 \\
0 \\
8 \\
0 \\
\dot{\forall}\end{array}$ & 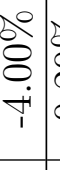 & 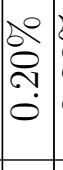 & 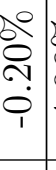 & $\mid \begin{array}{c}0 \\
0 \\
0 \\
\dot{+}\end{array}$ & $\begin{array}{c}\Delta \\
\vec{a} \\
\sigma \\
+ \\
+\end{array}$ & $\begin{array}{l}0 \\
\infty \\
\infty \\
\infty \\
\infty \\
\infty\end{array}$ & 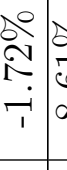 & \begin{tabular}{c|l}
0 & \\
0 & 0 \\
0 & 0 \\
$\infty$ & 0
\end{tabular} & 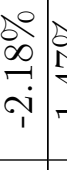 & 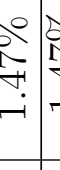 & 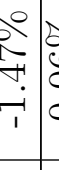 & 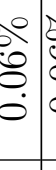 & $\begin{array}{l}0 \\
0 \\
0 \\
0 \\
0 \\
1\end{array}$ & 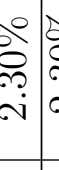 & 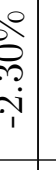 & \\
\hline & - & $\begin{array}{c}0 \\
-1 \\
-1\end{array}$ & $\begin{array}{l}50 \\
0 \\
0 \\
10 \\
0 \\
0\end{array}$ & $\begin{array}{l}0 \\
0 \\
0 \\
0 \\
0 \\
0 \\
1\end{array}$ & $\begin{array}{l}0 \\
0 \\
0 \\
0 \\
0 \\
0\end{array}$ & $\begin{array}{l}00 \\
0 \\
\infty \\
0 \\
0 \\
1\end{array}$ & 용 & 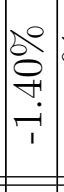 & $\begin{array}{c}0 \\
0 \\
0 \\
+ \\
+\end{array}$ & $\begin{array}{c}80 \\
8 \\
8 \\
+ \\
\dot{1}\end{array}$ & $\begin{array}{l}0 \\
\stackrel{0}{0} \\
\stackrel{N}{N} \\
0\end{array}$ & 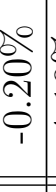 & 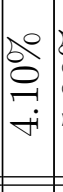 & $\begin{array}{c}8 \\
8 \\
8 \\
\dot{1} \\
1\end{array}$ & $\mid \begin{array}{c}0 \\
0 \\
0 \\
0 \\
0\end{array}$ & \begin{tabular}{l|l}
0 & \\
0 & \\
0 & 0 \\
0 &
\end{tabular} & 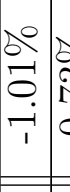 & 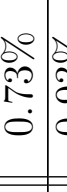 & 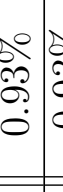 & $\begin{array}{l}0 \\
0 \\
0 \\
0 \\
0 \\
\\
1\end{array}$ & 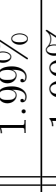 & $\begin{array}{l}0 \\
8 \\
0 \\
0 \\
\\
1 \\
1\end{array}$ & 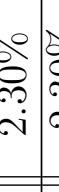 & 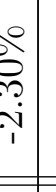 & \\
\hline & S & 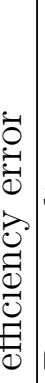 & 茪 & & 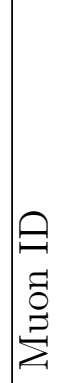 & & 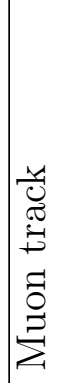 & & 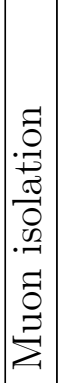 & & 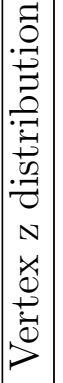 & & 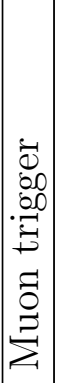 & & 玨 & & 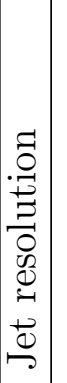 & & $\theta$ & & $\begin{array}{l}\overrightarrow{0} \\
0 \\
0 \\
0 \\
0 \\
0\end{array}$ & & 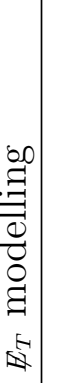 & & 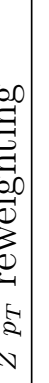 \\
\hline
\end{tabular}




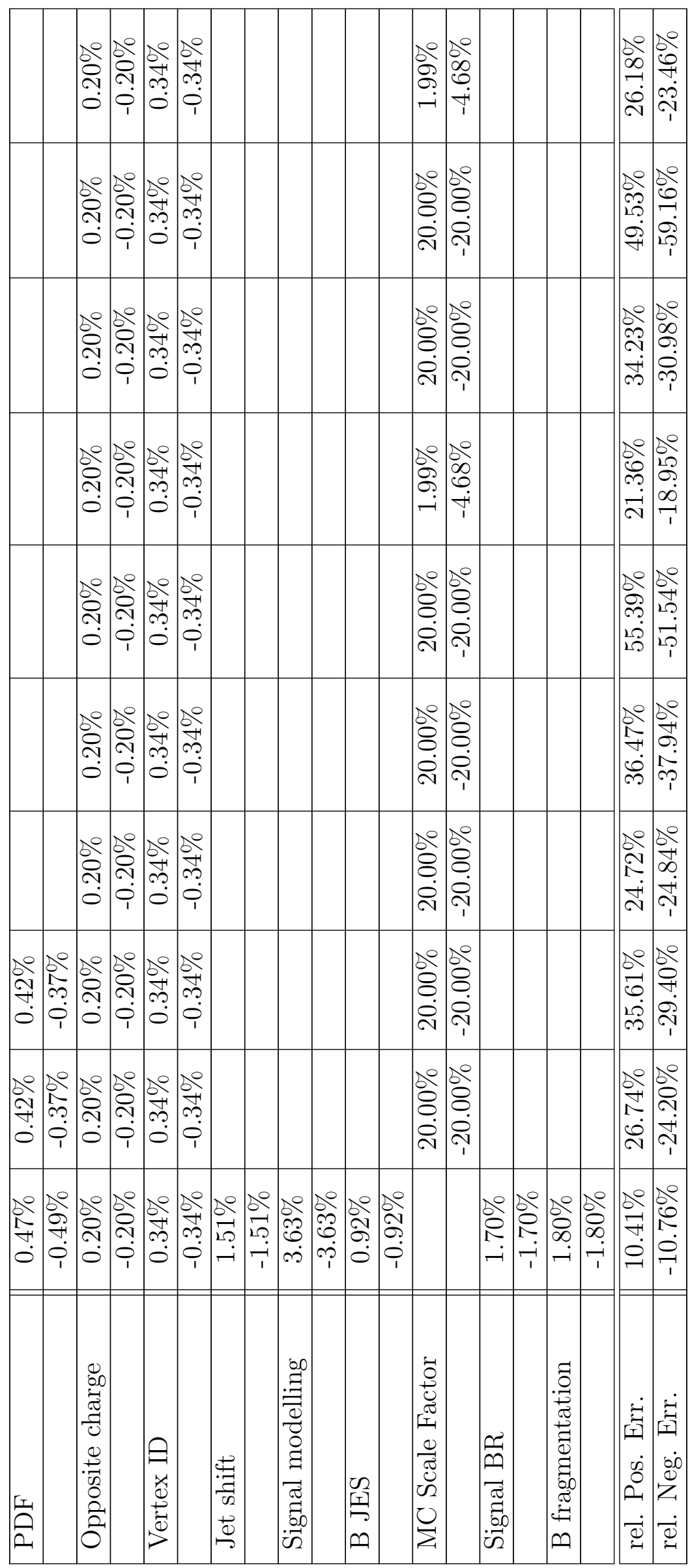


Table 6.15.: Summary of all sources of systematic uncertainties.

\begin{tabular}{c|c|c} 
Systematic & pos. error $/ \mathrm{pb}$ & neg. error $/ \mathrm{pb}$ \\
\hline Event preselection & 0.068 & -0.068 \\
Muon identification & 0.477 & -0.444 \\
Luminosity reweighting & 0.119 & -0.114 \\
$Z p_{T}$ reweighting & 0.243 & -0.243 \\
Dilepton trigger & 0.571 & -0.430 \\
Opposite charge requirement & 0.021 & -0.021 \\
Jet energy scale & 0.572 & -0.178 \\
Jet energy resolution & 0.117 & -0.368 \\
Met reconstruction and identification & 0.122 & -0.119 \\
M modelling & 0.251 & -0.240 \\
Monte Carlo background x-section & 0.246 & -0.120 \\
Monte Carlo signal \& bkg branching ratio & 0.088 & -0.085 \\
Monte Carlo bkg scale factors & 0.192 & -0.192 \\
Monte Carlo statistics & 0.667 & -0.667 \\
Instrumental background & 0.146 & -0.146 \\
PDF & 0.027 & -0.025 \\
signal modeling & 0.191 & -0.178 \\
Jet shifting & 0.078 & -0.075 \\
b-Jet energy scale & 0.047 & -0.046 \\
b fragmentation & 0.190 & -0.177 \\
Luminosity & 0.330 & -0.292 \\
\hline Total systematic & 1.347 & -1.175 \\
Total systematic w/o lumi & 1.306 & -1.138
\end{tabular}

\subsection{Results}

As can be seen from Table 6.16, 12 events were selected after all cuts. The procedure described in Section 6.3.6 gives the number of "fake" muon background events. The number of $t \bar{t}$ events is obtained after subtraction of the fake muon background and the MC predictions for the "physics" backgrounds ( $W W, W Z, Z Z, Z \rightarrow \mu \bar{\mu}$ and $Z \rightarrow \tau \bar{\tau}$ ) from the fit results for signal events. The details on the events yield and MC background predictions for the 0-, 1- and 2-jets inclusive multiplicity as well as for the final selection are shown in Table 6.7 .

To estimate the cross-section $\sigma$ using the selection efficiency $\epsilon$, the branching ratio BR, the integrated luminosity $\mathcal{L}$ and the number of estimated background events $N^{b k g}$ we define the following likelihood function:

$$
L\left(\sigma, N^{o b s}, N^{b k g}, B R, \mathcal{L}, \epsilon\right)=\mathcal{P}\left(N^{o b s}, \mu\right),
$$

where $\mathcal{P}\left(N^{o b s}, \mu\right)$ is the Poisson probability of expected $\mu$ signal-plus-background events to be compatible with the number of events observed in data, $N^{\text {obs }}$,

$$
\mathcal{P}\left(N^{o b s}, \mu\right)=\frac{\mu^{N^{o b s}}}{N^{o b s} !} \exp ^{-\mu}
$$


Table 6.16.: Expected background, observed and expected signal yield for $t \bar{t} \rightarrow \mu \mu$ channel.

\begin{tabular}{c|c|c|c}
\hline Category & Yield & Stat Err & Sys Err \\
\hline$W W$ & 0.31 & \pm 0.05 & ${ }_{-0.07}^{+0.08}$ \\
$W Z$ & 0.13 & \pm 0.03 & ${ }_{-0.04}^{+0.05}$ \\
$Z Z$ & 0.12 & \pm 0.01 & ${ }_{-0.03}^{+0.05}$ \\
$Z / \gamma^{*} \rightarrow \mu \bar{\mu}$ & 4.4 & \pm 0.72 & ${ }_{-0.94}^{+1.77}$ \\
$Z / \gamma^{*} \rightarrow \tau \bar{\tau}(\tau \rightarrow \mu)$ & 1.24 & \pm 0.17 & ${ }_{-0.31}^{+0.52}$ \\
Muon fake & 0.16 & \pm 0.16 & \\
\hline Total Bkg & 6.36 & \pm 0.47 & ${ }_{-0.99}^{+1.85}$ \\
\hline Expected signal & 8.79 & \pm 0.10 & ${ }_{-0.01}^{+0.01}$ \\
\hline Expected signal+background & 15.15 & \pm 0.57 & ${ }_{-0.99}^{+1.85}$ \\
\hline Selected Events & 12 & \pm 3.46 & \\
\hline
\end{tabular}

The sum of expected signal-plus-background is given by

$$
\mu=\sigma \times B R \times \mathcal{L} \times \epsilon+N^{b k g} .
$$

Using the efficiency from Table 6.6 (6.85\%) assuming a top mass of $170 \mathrm{GeV}$, the integrated luminosity of $1008.96 \mathrm{pb}^{-1}$ and the $\mu \bar{\mu}$ branching ratio of $(0.12675)^{2}$ for the $t \bar{t} \rightarrow \mu \bar{\mu}$ process (including the cascade decay $t \rightarrow \tau \rightarrow \mu$ ), the $p \bar{p} \rightarrow t \bar{t}$ cross-section is found to be:

$$
\mu \bar{\mu}: \quad \sigma_{t \bar{t}}=5.08_{-2.83}^{+3.43} \text { (stat) }{ }_{-1.14}^{+1.31} \text { (syst) }{ }_{-0.61}^{+0.69} \text { (lumi) pb. }
$$

This measurement can be compared with the theoretical predictions of Cacciari et al. and Moch et. al. in Tab. 2.4 and Fig. 2.4. Fig. 6.26 shows the same as Fig. 2.4, but this time overlaid with the measured value and its dependence on the top mass.

The error band shown for the dependence of the measured cross-section on the top mass contains the statistical as well as the systematic uncertainty. In the latter case, the uncertainty was scaled with the ratio of the cross-section for $m_{\text {top }}=170 \mathrm{GeV}$ to the crosssection for any other value of the top mass. The dependence on the top quark mass as well as the uncertainty dependence are parametrized by

$$
\sigma\left(m_{\text {top }}\right)=a+b\left(m_{\text {top }}-130 G e V\right)+c e^{d\left(m_{t o p}-130 G e V\right)}
$$

with $\mathrm{a}=8.09 \mathrm{pb}, \mathrm{b}=-3.20 \times 10^{-2} \mathrm{pb} / \mathrm{GeV}, \mathrm{c}=6.44 \mathrm{pb}$ and $\mathrm{d}=6.08 \times 10^{-2} \mathrm{GeV}^{-1}$ for the central value. For the lower and upper bounds on the uncertainty, the parameters are $\mathrm{a}=$ $6.61 \mathrm{pb}, \mathrm{b}=-2.69 \times 10^{-2} \mathrm{pb} / \mathrm{GeV}, \mathrm{c}=5.23 \mathrm{pb}$ and $\mathrm{d}=6.13 \times 10^{-2} \mathrm{GeV}^{-1}$ and $\mathrm{a}=9.73 \mathrm{pb}, \mathrm{b}$ $=-3.80 \times 10^{-2} \mathrm{pb} / \mathrm{GeV}, \mathrm{c}=7.74 \mathrm{pb}$ and $\mathrm{d}=6.10 \times 10^{-2} \mathrm{GeV}^{-1}$, respectively. The fit function, Eq. 6.12, was offset by $130 \mathrm{GeV}$ to avoid large values of the exponential. This improves the stability of the fit and the error of the fit parameters.

\subsubsection{Combination with other Dilepton Cross Section Analyses}

The measurement of the top quark pair production cross section has been measured in events with two identified electrons, or an electron-muon pair. Though these analyses are 


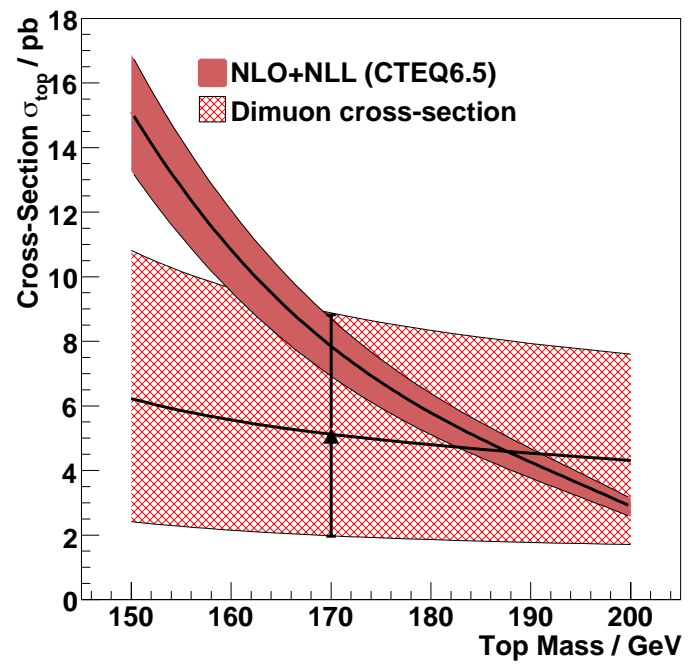

(a) Cacciari et. al. (CTEQ6.5)

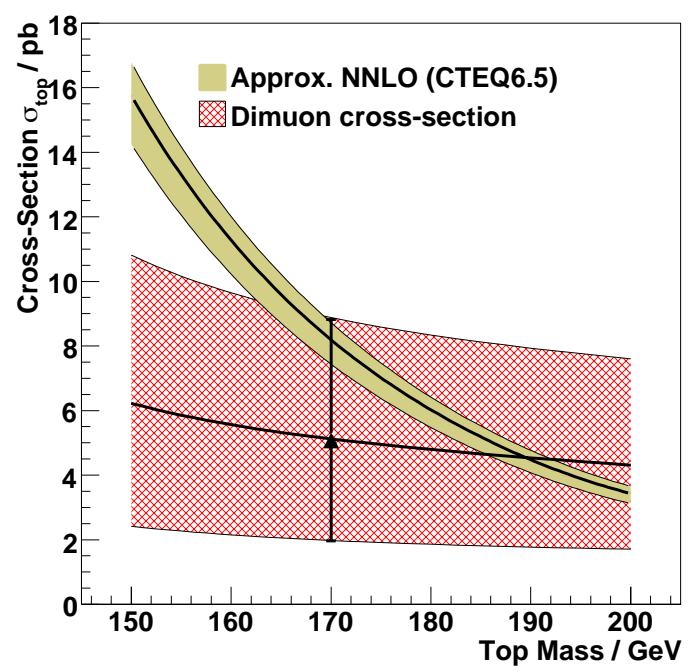

(c) Moch et. al. (CTEQ6.5)

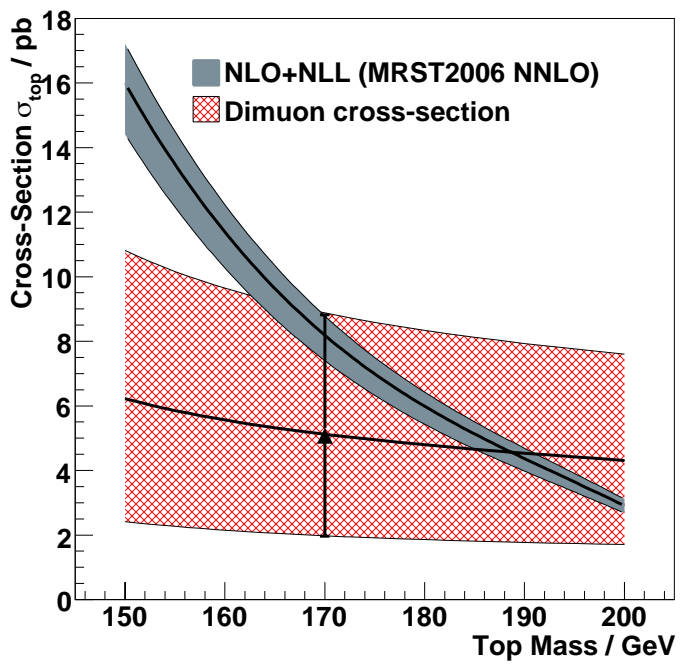

(b) Cacciari et. al. (MRST 2006 NNLO)

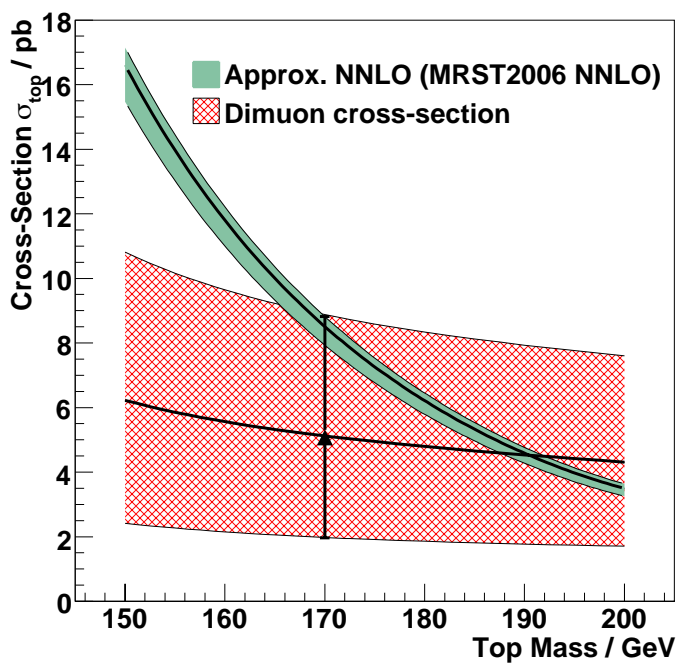

(d) Moch et. al. (MRST 2006 NNLO)

Figure 6.26.: Dependence of the top quark pair production cross-section in the dimuon final state on the top mass. The single data point at $m_{\text {top }}=170 \mathrm{GeV}$ shows the measured value and its error. 
not the subject of this thesis, we briefly describe these measurements and the combination of the $e e$ and the $e \mu$ cross section ([97] and [96]) with our result in this section.

As for the dimuon final state, the event selection in the $e e$ and $e \mu$ channel starts with the selection of two high- $p_{T}$ isolated and oppositely charged leptons with $p_{T}>15 \mathrm{GeV}$, and two jets. The leptons have to be within the acceptance of the detectors. For muons, the requirement is thus $|\eta|<2.0$ as mentioned in Sec. 6.3.1, while for electrons $|\eta|<1.1$ or $1.5<|\eta|<2.5$. The leading jet is required to have a $p_{T}$ greater than $30 \mathrm{GeV}$ and $p_{T}^{\text {second jet }}>20 \mathrm{GeV}$. To reduce the $Z$ background in the ee channel, events with dielectron invariant mass $M_{e e}<15 \mathrm{GeV}$ or $84<M_{e e}<100 \mathrm{GeV}$ are rejected. Furthermore, the events are required to have $E_{T}>35 \mathrm{GeV}$ for $M_{e e}>100 \mathrm{GeV}$ and $E_{T}>40 \mathrm{GeV}$ for $15<M_{e e}<84 \mathrm{GeV}$. In the e $\mu$ channel, $H_{T}>115 \mathrm{GeV}$ concludes the event selection. Here, $H_{T}$ is the sum of the leading lepton and all jet transverse momenta.

In addition to the cross-section measurement in the two jet bin, the cross-section in $\mathrm{e} \mu$ analysis has been determined in the one jet exclusive bin, i.e. for events that satisfy $N_{\text {jets }}=1$. This result has then been combined with the measurement in the two jet bin, i.e. $N_{\text {jets }} \geq 2$.

In the dielectron channel, the number of selected events is 17, with 3.4 expected background events. Assuming a top mass of $170 \mathrm{GeV}$, the cross section in the dielectron channel is

$$
\mathrm{ee}: \quad \sigma_{t t}=9.6_{-2.7}^{+3.2}(\text { stat })_{-0.9}^{+1.0}(\text { syst })_{-0.7}^{+0.8}(\text { lumi }) p b
$$

In the electron-muon final state, the combined cross-section result of the one jet exclusive and two jet inclusive measurements is

$$
\mathrm{e} \mu \text { combined : } \quad \sigma_{t t}=7.2_{-1.3}^{+1.4}(\text { stat })_{-0.7}^{+0.8}(\text { syst }) \pm 0.6(\text { lumi }) p b
$$

The combined cross section is extracted minimizing the following likelihood function:

$$
L\left(\sigma,\left\{N_{j}^{o b s}, N_{j}^{b k g}, B R_{j}, \mathcal{L}_{j}, \epsilon_{j}\right\}_{j=1 \ldots n}\right)=\prod_{j} \frac{\left(\sigma B R_{j} \mathcal{L}_{j} \epsilon_{j}+N_{j}^{b k g}\right)^{N_{j}^{o b s}}}{N_{j}^{o b s !}} \exp ^{-\left(\sigma B R_{j} \mathcal{L}_{j} \epsilon_{j}+N_{j}^{b k g}\right)}
$$

which is the product of the likelihood in the individual channels. Using the number of observed events in data and the number of expected background events from each channel, the combined cross-section is found to be

$$
\text { dilepton : } \quad \sigma_{t t}=7.4_{-1.1}^{+1.2}(\text { stat })_{-0.9}^{+1.0}(\text { syst }),
$$

where the uncertainty on the integrated luminosity is contained in the systematic uncertainty. Again, the cross-section is dependent on the assumed top mass. The variation of the combined dilepton cross-section as a function of $m_{t o p}$ is shown in Fig. 6.27.

As for the dimuon cross-section, the dependence on the top quark mass is parametrized by

$$
\sigma\left(m_{\text {top }}\right)=a+b\left(m_{\text {top }}-130 G e V\right)+c e^{d\left(m_{\text {top }}-130 G e V\right)}
$$

with $\mathrm{a}=8.09 \mathrm{pb}, \mathrm{b}=-3.20 \times 10^{-2} \mathrm{pb} / \mathrm{GeV}, \mathrm{c}=6.44 \mathrm{pb}$ and $\mathrm{d}=6.08 \times 10^{-2} \mathrm{GeV}^{-1}$. The same parametrization was used for the error band, but now the parameters are $\mathrm{a}=6.61$ $\mathrm{pb}, \mathrm{b}=-2.69 \times 10^{-2} \mathrm{pb} / \mathrm{GeV}, \mathrm{c}=5.23 \mathrm{pb}$ and $\mathrm{d}=6.13 \times 10^{-2} \mathrm{GeV}^{-1}$ for the lower bound and $\mathrm{a}=9.73 \mathrm{pb}, \mathrm{b}=-3.80 \times 10^{-2} \mathrm{pb} / \mathrm{GeV}, \mathrm{c}=7.74 \mathrm{pb}$ and $\mathrm{d}=6.10 \times 10^{-2} \mathrm{GeV}^{-1}$ for the upper bound of the uncertainty. 
Because of the small error band on the combined dilepton cross-section, it is the best opportunity to extract the top mass based on these results as described in the next section.

\subsubsection{Determination of the Top Quark Mass from the Production Cross Section}

The concept of a quark mass is convention dependent. Thus, the value of the top quark mass can vary considerably for different definitions such as $\overline{M S}$ mass or pole mass. Current methods of the top quark mass measurements including neutrino weighting [98] and matrix element [99] methods rely strongly on the detailed simulation of the top pair production signal and thus on the mass definition assumed. Since all currently used Monte Carlo simulations contain matrix elements in leading order QCD, while higher orders are simulated through parton shower methods, the convention used for the top quark mass is in principle unknown.

Independent and valuable information about the top mass can be gained from the crosssection measurements which have the advantage not to rely on the simulation of the signal except for the determination of signal selection efficiencies. Since NLO corrections to the $t \bar{t}$ signal affect mostly the normalization, we assume that the signal selection efficiencies are not affected much.

From the intersection of the measured cross-section curves with the theoretical predictions the top quark mass is extracted. It is assumed that the experimental and theoretical uncertainties are not correlated, and we quote them separately. This is justified as the NLO corrections affect the normalization of the signal rather than the shape of kinematic distributions.

The top quark mass extraction of [3] has been updated to the most recent theory predictions for the top quark production cross-section. The combined cross-section as described in the previous section is compared to the aforementioned calculations of the top quark pair production cross-section carried out by Cacciari et. al [46] and Moch and Uwer [43]. The first performed at NLO QCD with NLL threshold corrections, the latter includes scaleindependent threshold corrections up to NNLL and the known scale-dependent terms at NNLO [43]. These calculations were performed using the top quark pole mass and represent the most accurate calculation of the $t \bar{t}$ cross-section to date.

The comparison of the experimental and theoretical results yields a top mass of

$$
m_{\text {top }}=172.80_{-8.38}^{+9.37}(\text { stat }+ \text { syst })_{-5.67}^{+4.47}(\text { theory }) \mathrm{GeV}(\text { CTEQ6.5) }
$$

and

$$
m_{\text {top }}=174.52_{-8.09}^{+9.03}(\text { stat }+ \text { syst })_{-4.37}^{+3.05}(\text { theory }) \text { GeV (MRST2006 NNLO) }
$$

using Cacciari et. al. and

$$
m_{\text {top }}=174.68_{-8.37}^{+9.25}(\text { stat }+ \text { syst })_{-4.29}^{+2.77}(\text { theory }) \mathrm{GeV}(\text { CTEQ6.5) }
$$

and

$$
m_{\text {top }}=176.17_{-8.08}^{+8.97}(\text { stat }+ \text { syst })_{-3.02}^{+1.58}(\text { theory }) \mathrm{GeV}(\text { MRST2006 NNLO })
$$




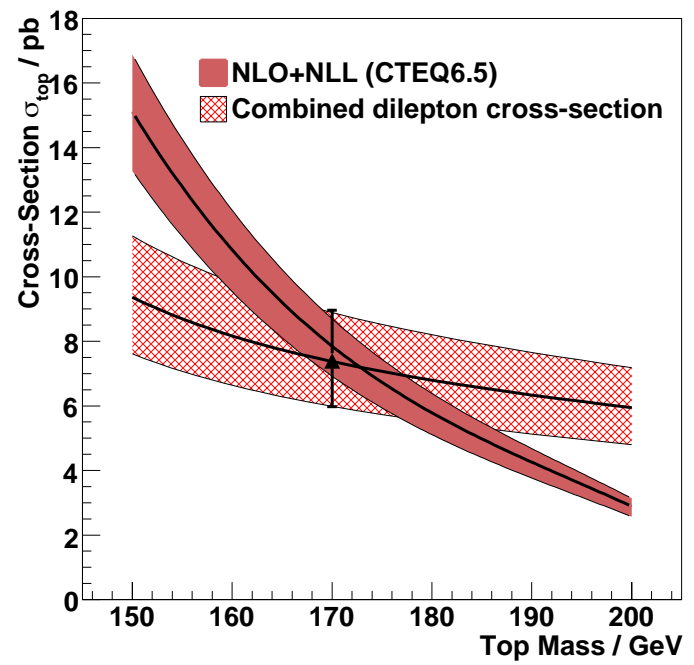

(a) Cacciari et. al. (CTEQ6.5)

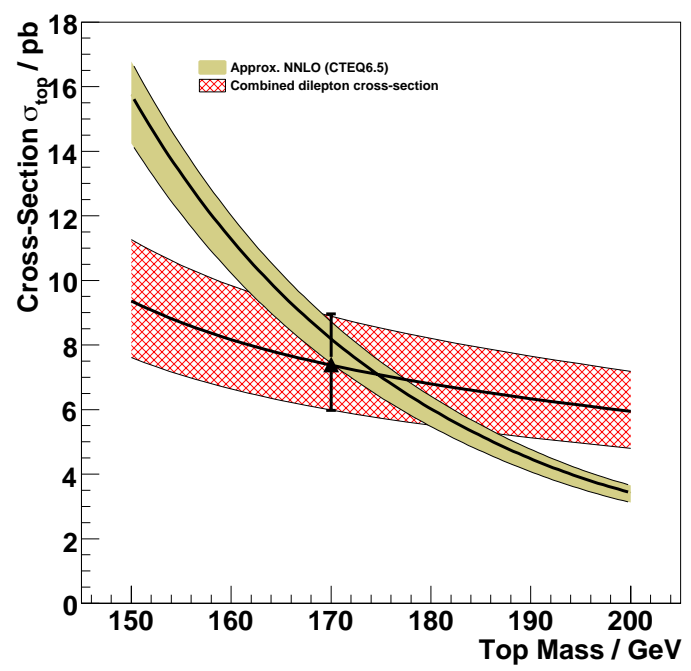

(c) Moch et. al. (CTEQ6.5)

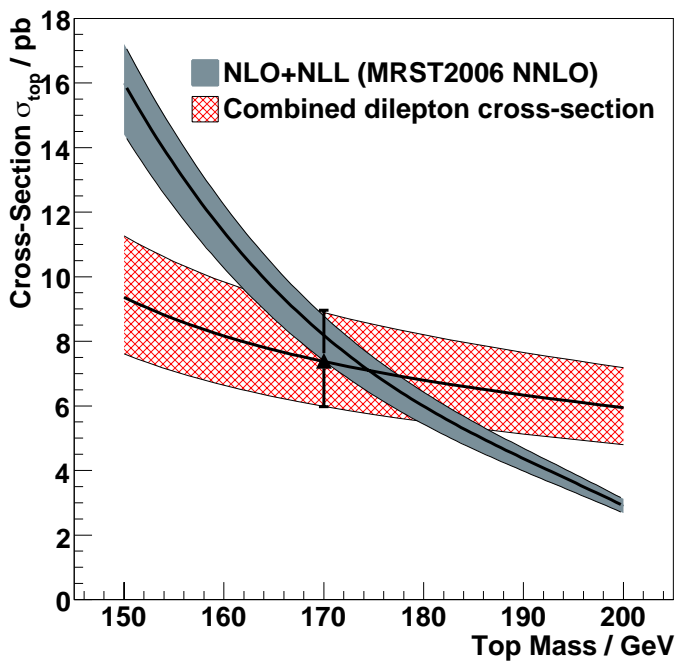

(b) Cacciari et. al. (MRST 2006 NNLO)

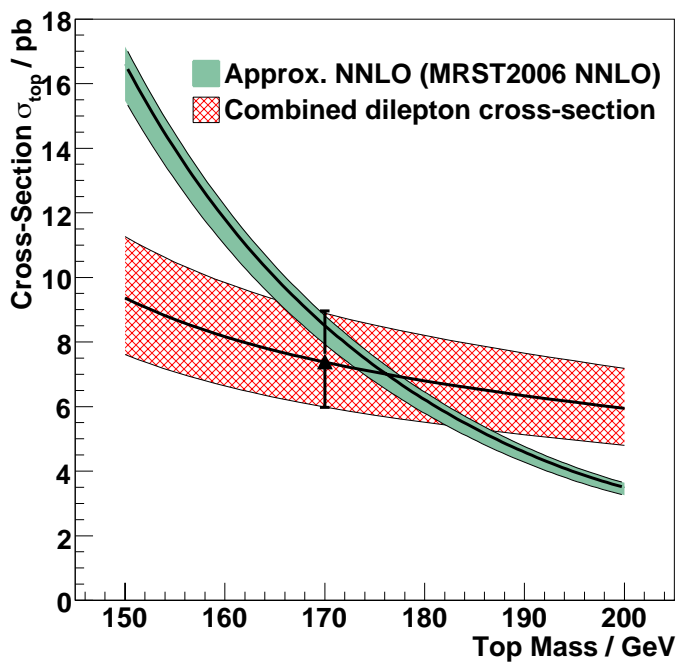

(d) Moch et. al. (MRST 2006 NNLO)

Figure 6.27.: Dependence of the combined dilepton top quark pair production cross-section on the top mass. The single data point at $m_{t o p}=170 \mathrm{GeV}$ shows the combined cross-section and its error. 
using Moch and Uwer. Fig. 6.28 shows the measured cross section and the theoretical calculation as a function of top mass.

These values are in accordance with the respective mass measurements in the dilepton final states using the neutrino weighting [98] and matrix weighting [99] methods,

$$
m_{\text {top }}=175.2 \pm 7.0 \mathrm{GeV}(\text { stat }+ \text { syst })
$$

for the matrix weighting and

$$
m_{\text {top }}=172.5 \pm 6.77 \mathrm{GeV}(\text { stat }+ \text { syst })
$$

for the neutrino weighting method. All values are in agreement with the current world average for the top quark mass [100],

$$
m_{\text {top }}=172.4 \pm 1.4 \mathrm{GeV} \text { (stat }+ \text { syst) }
$$

However, it has to be emphasized that the definition of the top quark mass extracted in cross section measurements is not identical to the one extracted using event kinematics. Complementary information has been derived by two means. First, complementary experimental information has been used to derive a value for the top mass, and second, a different quantity for the top mass has been extracted compared to the mass measurements based on event kinematics.

A different approach to estimate the errors associated with the top mass derived from cross-section measurements was presented in [101]. The experimental cross-section was parametrized as a third-order polynomial in the top quark mass. Using this parametrization together with the parametrizations of the theory prediction as given in [46] and [43], likelihoods are defined as a function of the top quark pair production cross-section and the top quark mass. These two likelihoods are then multiplied and integrated in the cross-section. Thus, the likelihood function depends only on the top quark mass, and the extracted mass is given by the minimum of the likelihood function. This method was applied to the combined dilepton, tau+lepton and lepton+jets cross-section derived in [7],

$$
\sigma_{p \bar{p} \rightarrow t \bar{t}}=7.83_{-0.70}^{+0.77} \text { (stat. + syst.) } \pm 0.48 \text { (lumi)pb }
$$

to give

$$
m_{\text {top }}=167.8_{-5.7}^{+5.7} \mathrm{GeV} \text { (theory + experiment; CTEQ6.6) }
$$

using the calculation from Cacciari et al. and

$$
m_{\text {top }}=169.6_{-5.5}^{+5.4} \mathrm{GeV} \text { (theory + experiment; CTEQ6.6) }
$$

using the calculation from Moch\&Uwer. 


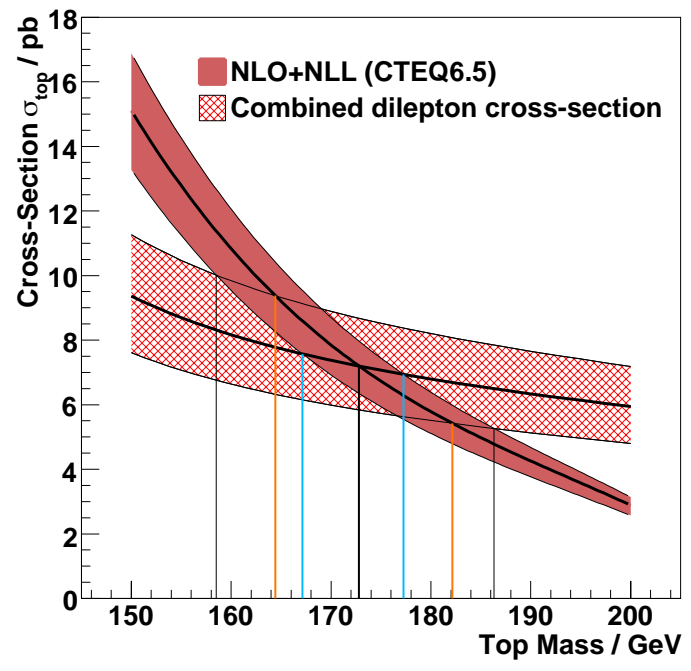

(a) Cacciari et. al. (CTEQ6.5)

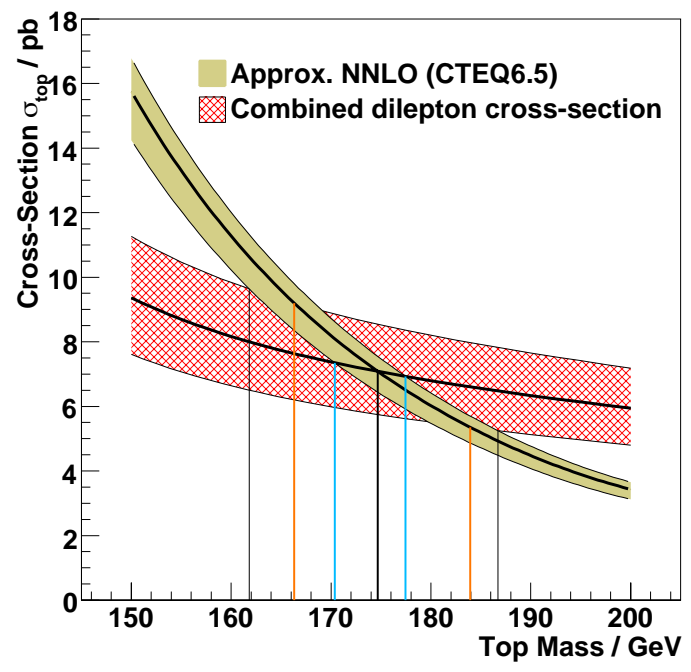

(c) Moch et. al. (CTEQ6.5)

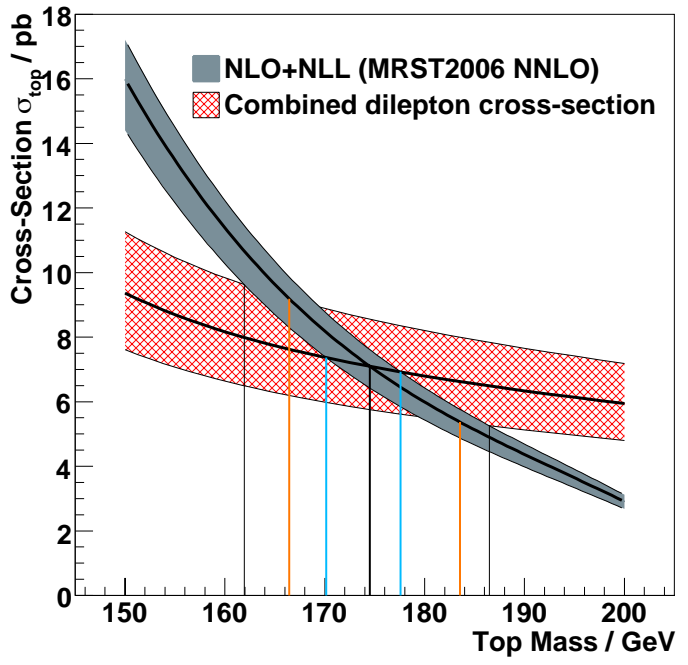

(b) Cacciari et. al. (MRST 2006 NNLO)

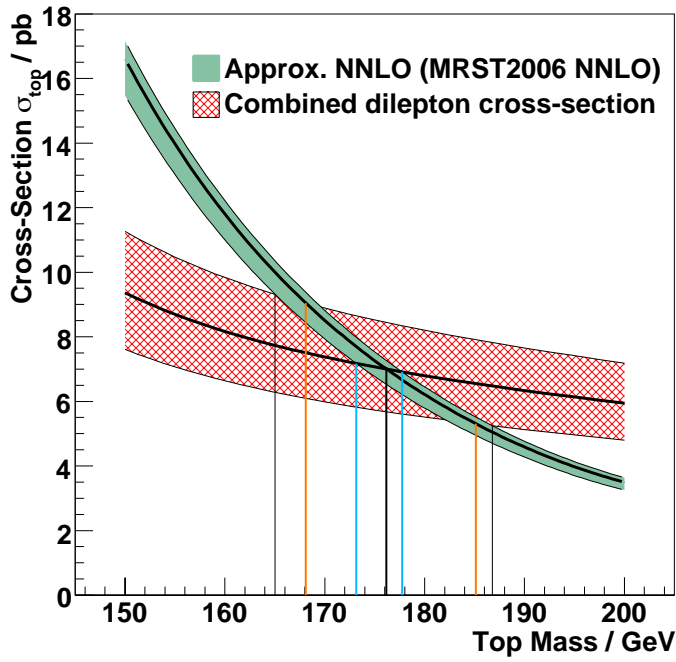

(d) Moch et. al. (MRST 2006 NNLO)

Figure 6.28.: The combined dilepton top quark production cross section overlaid with different theory predictions. The blue vertical lines indicate the uncertainty of $m_{t o p}$ induced by the theoretical uncertainty. The orange lines indicate the uncertainty of $m_{t o p}$ due to the combined statistical and systematic uncertainty of the cross-section measurement. 


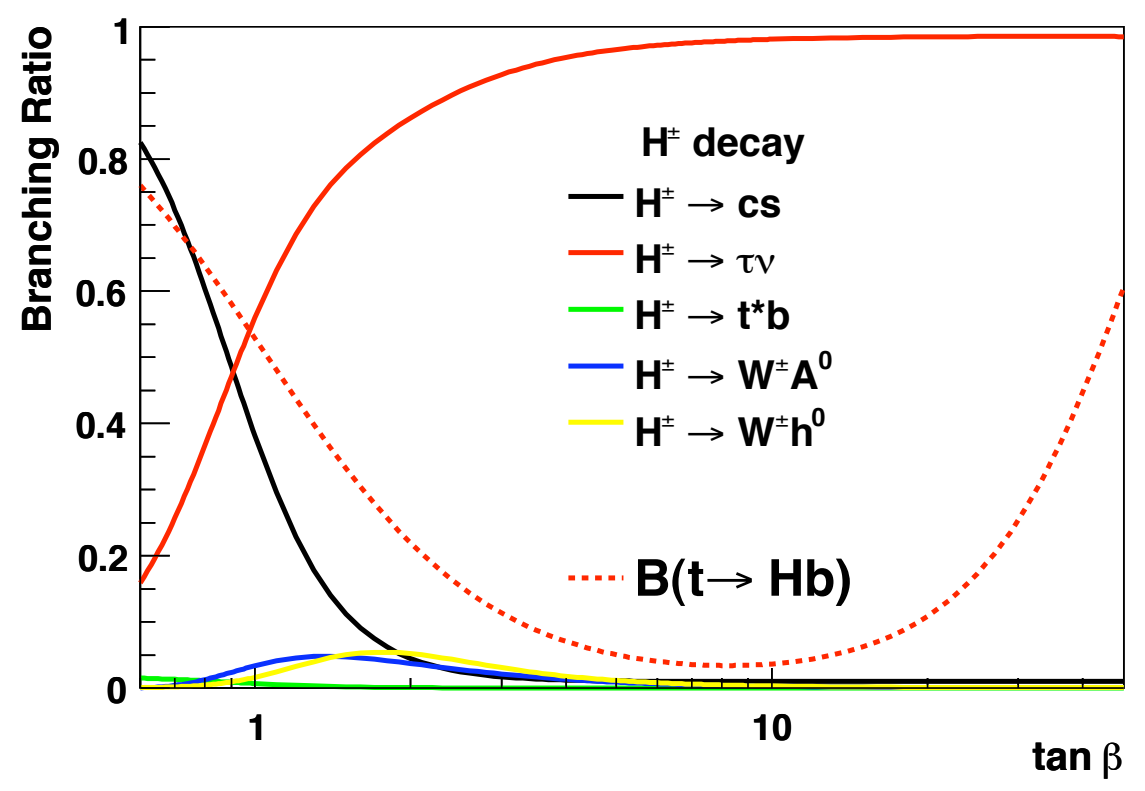

Figure 6.29.: The branching ratio for top decays assuming that the only decay modes are $t \rightarrow W^{+} b$ and $t \rightarrow H^{+} b$ as calculated with CPsuperH [103] (as found in [7]).

\subsubsection{Charged Higgs Search}

Many extensions of the Standard Model, such as Supersymmetry 102 and Grand Unified Theories (GUTs), require the existence of an additional Higgs doublet. These models predict additional physical Higgs particles, including two charged Higgs bosons.

For a charged Higgs mass smaller than the top quark mass, the branching fraction of the top decay to charged Higgs can be sizable. In the context of the Minimal Supersymmetric Standard Model (MSSM), the dominant decay channels for a charged Higgs are $H^{ \pm} \rightarrow \tau \nu_{\tau}$ for large $\tan (\beta)$, and $H^{ \pm} \rightarrow c s$ for small $\tan (\beta)$ and $H^{ \pm}$masses, where $\tan (\beta)$ is the vacuum expectation value of the two Higgs doublets in the MSSM. For small $\tan (\beta)$ and large $H^{ \pm}$masses, $H^{ \pm} \rightarrow t b$ is the dominant decay mode. Fig. 6.29 shows the branching ratios for different charged Higgs decay modes as a function of $\tan \beta$ assuming a charged Higgs mass of $100 \mathrm{GeV}$.

The dilepton final state was included in a recent charged Higgs particle search [7]. In addition to the SM decay $t \rightarrow W b$, the decay of the top quark to a charged Higgs boson is included, and both bosons ( $W$ and $H$ ) decay into a light lepton.

In the presence of top quark to charged Higgs decays, one expects a decrease of $\sigma_{t \bar{t} \rightarrow \text { dilepton }}$ and $\sigma_{t \bar{t} \rightarrow l+j e t s}$ ("disappearance channels") for most regions of $\tan (\beta)$ and an increase in both the $\tau+$ jets and $\tau+$ lepton final states ("appearance channels") for $H^{ \pm} \rightarrow \tau \nu$. For $H^{ \pm} \rightarrow c s$, all final states except the all-hadronic final state are disappearance channels. As the decrease is different for $\sigma_{t \bar{t}_{\rightarrow \text { dilepton }}}$ and $\sigma_{t \bar{t} \rightarrow l+j e t s}$, the ratio of these two cross-sections can be used to set a limit on beyond the Standard Model decay modes of the top quark, $t \rightarrow b H^{ \pm}$being one example.

By assuming the SM top quark pair production cross section, we can look for deviations of the prediction from data in the $\mu \mu$ and other channels by performing a global fit to the production cross section measured in the different final states. 
As described in [7], the top quark production cross section has been fixed to $\sigma_{t \bar{t}}=$ $7.3 \pm 0.7 p b$. In order to extract the branching ratio of a top quark decaying to charged Higgs and a b-quark the expected number of events in the dilepton final states was calculated before performing a maximum likelihood fit to the number of observed events in data. The procedure is much the same to the combination of the dilepton cross-sections, with the exception that the branching ratio of $t \rightarrow H^{+} b$ is now the free parameter in the fit. Then the limits on $B R\left(t \rightarrow H^{ \pm} b\right)$ are extracted using prescription of Feldman and Cousins [104]. It is also possible to fit the top quark production cross-section and $B R(t \rightarrow$ $\left.H^{+} b\right)$ simultaneously. However, cross-section measurements in both disappearance and appearance channels are needed for this [7]. Combining the top quark pair production cross-section measurements in the dilepton, tau+lepton and lepton + jets final states in this simultaneous fit, the limits shown in Fig. 6.32 can be set depending on $\tan (\beta)$ and the charged Higgs mass. Adding limits from the leptophobic model leads to the exclusion plot shown in Fig. 6.33. 


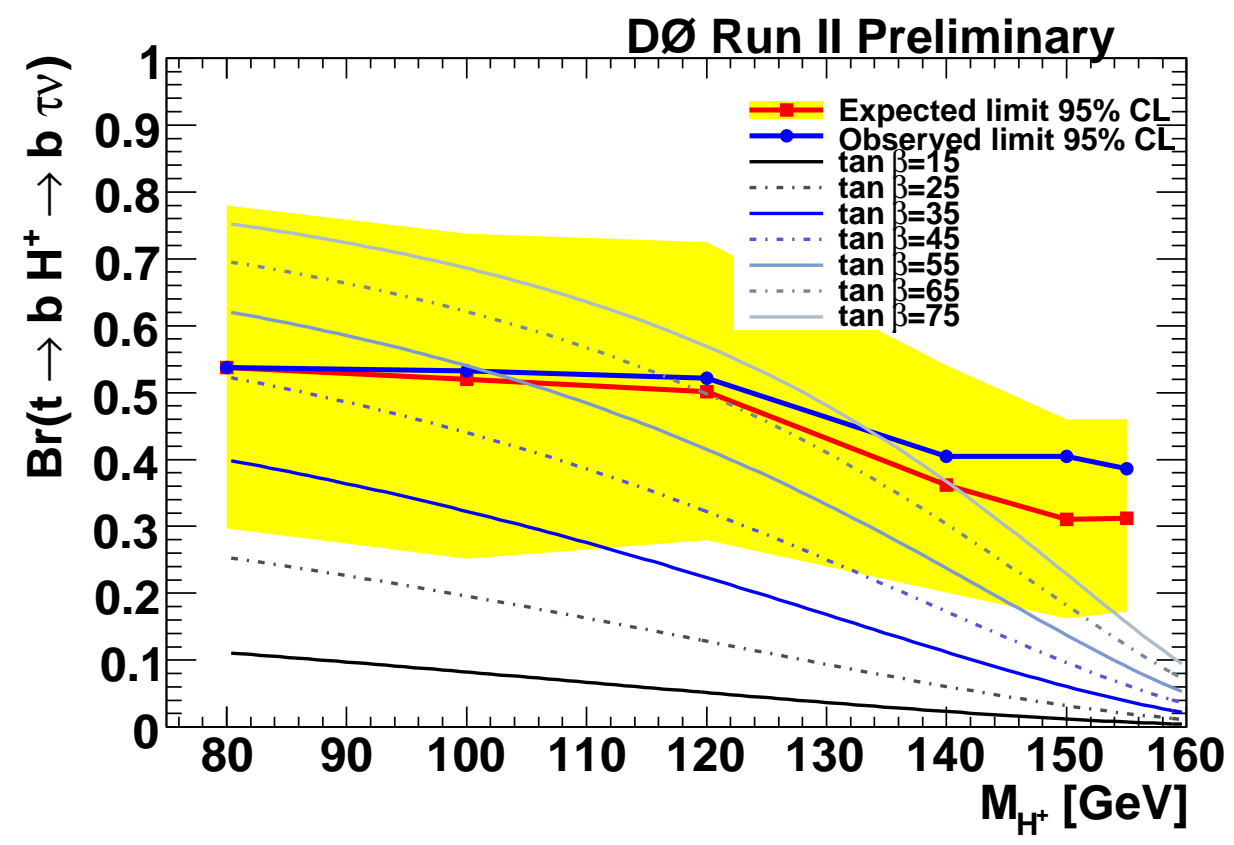

Figure 6.30.: The expected and observed limit on the branching ratio of $t \rightarrow H^{+} b \rightarrow \tau^{+} \nu b$ as a function of the charged Higgs mass in the tauonic model. The solid and dashed lines indicate predictions for $\mathrm{BR}\left(t \rightarrow H^{+} b\right)$ for various values of $\tan (\beta)$. The yellow band indicates the Feldman-Cousins $95 \%$ confidence level band.

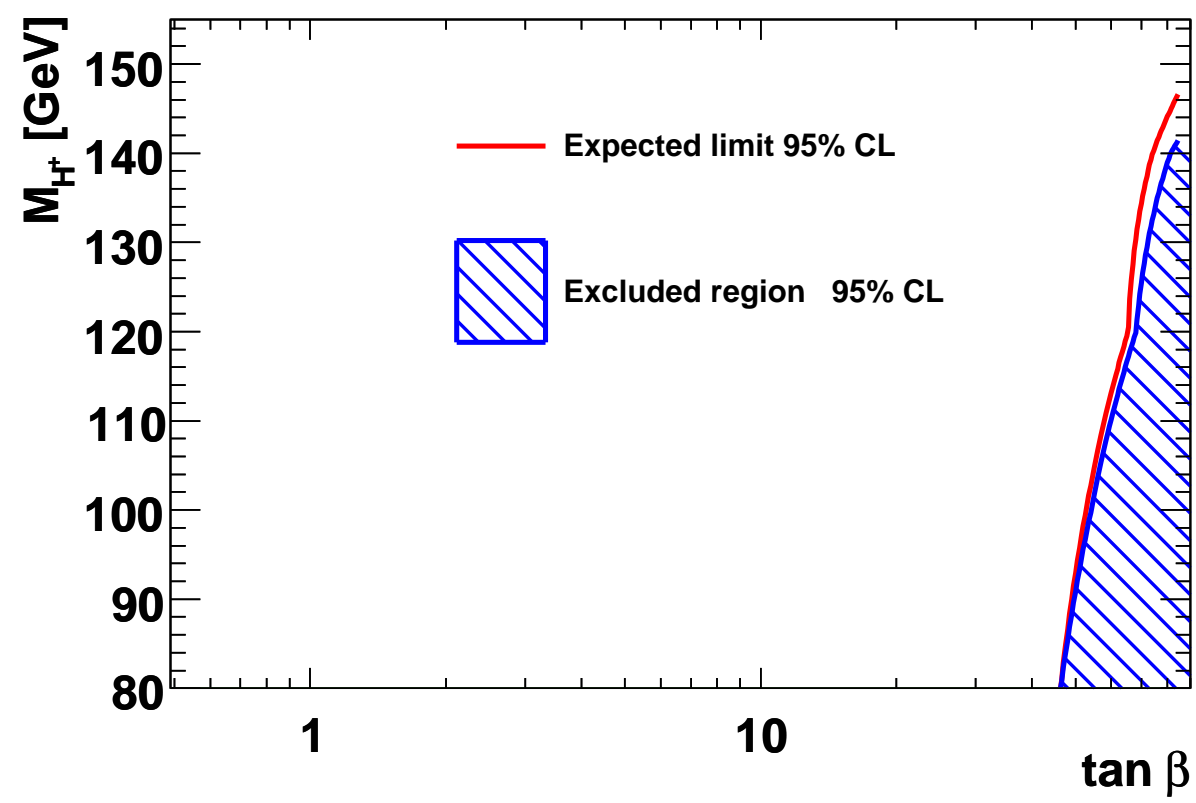

Figure 6.31.: The observed and expected limit in the $m_{H^{+}}$vs. $\tan (\beta)$ plane. The hashed area indicates the region in this plane that is excluded by this search. 


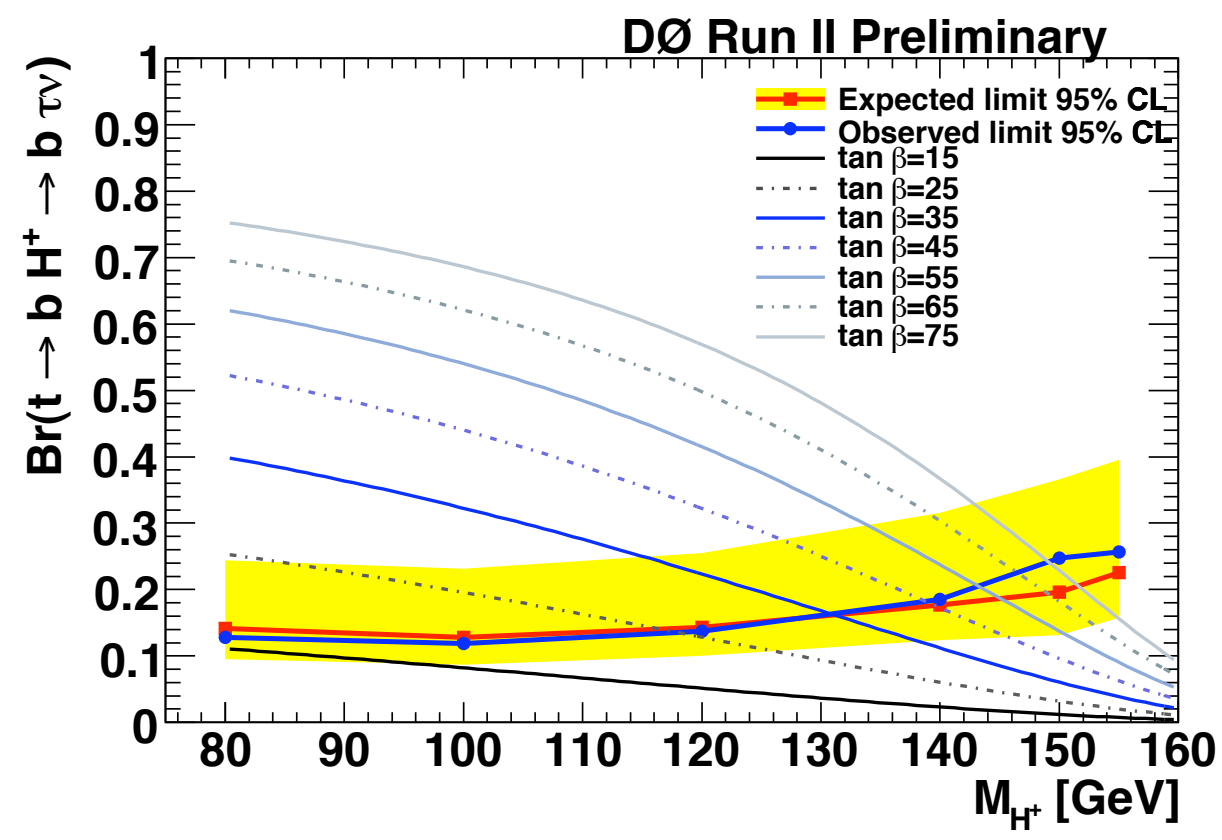

Figure 6.32.: The expected and observed limit on the branching ratio of $t \rightarrow H^{+} b \rightarrow \tau^{+} \nu b$ as a function of the charged Higgs mass for simultaneous fit of $\operatorname{BR}(t \rightarrow$ $\left.H^{+} b\right)$ and $\sigma_{p \bar{p} \rightarrow t \bar{t}}$ in the tauonic model. The solid and dashed lines indicate predictions for $\operatorname{BR}\left(t \rightarrow H^{+} b\right)$ for various values of $\tan (\beta)$. The yellow band indicates the Feldman-Cousins $95 \%$ confidence level band.

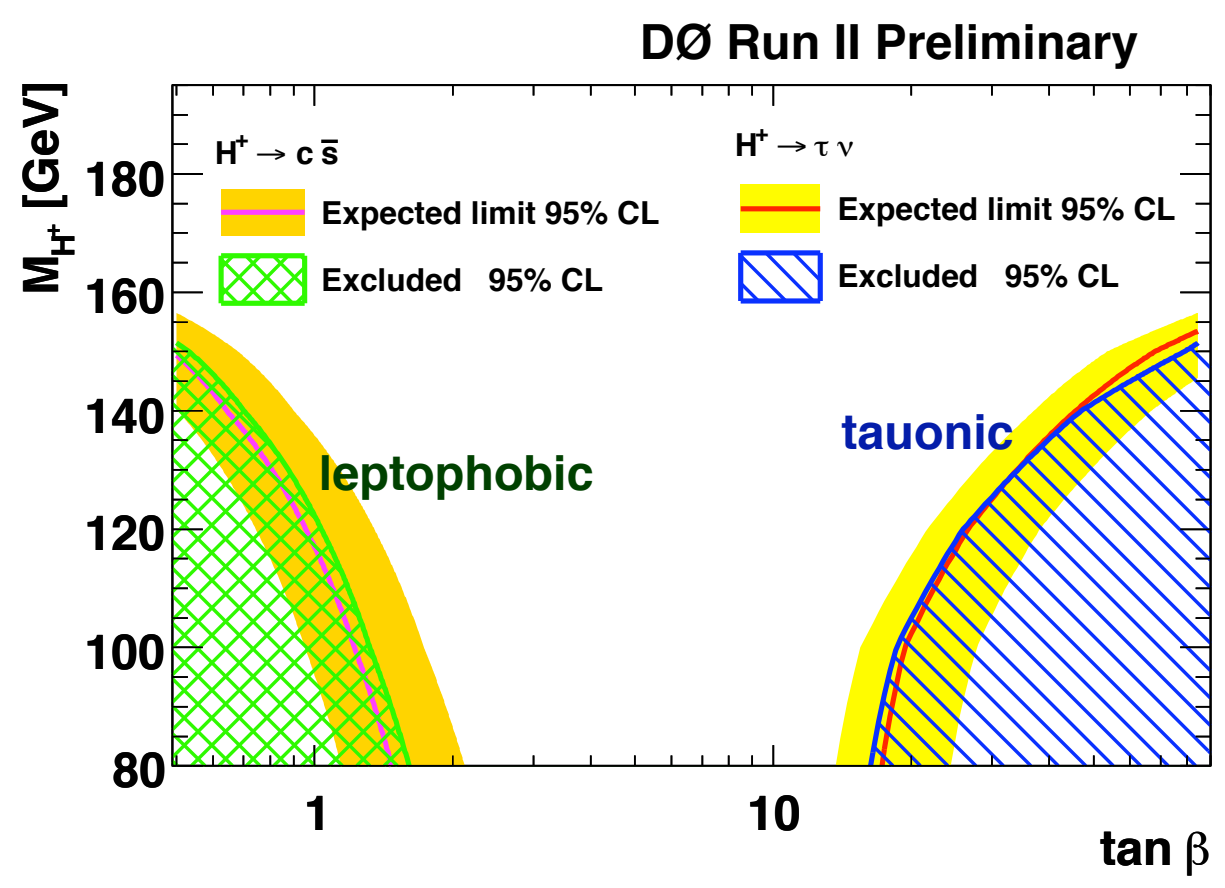

Figure 6.33.: The observed and expected limit in the $m_{H^{+}}$vs. $\tan (\beta)$ plane derived with the simultaneous fit. The hashed area indicates the region in this plane that is excluded by this search. 


\section{Monte Carlo Studies of the Top - Anti-Top Spin Correlation in the Dimuon Final State}

\subsection{Reconstruction of Dilepton Events}

To measure the spin correlation in $t \bar{t}$ events, ideally all objects in the event are identified and the lepton+jets final state would be the natural choice. However, using $t \bar{t}$ events with this final state requires identification of the down-type quark to measure the spin correlation, which is very difficult. Therefore we measure the spin correlation in the dilepton final state. The charged leptons are the most sensitive probes to $t \bar{t}$ spin correlation. In addition, to identify a b-jet no b-tagging is necessary, since there are only 2 jets at leading order. However, the statistics in the dilepton final states are smaller than in the $1+$ jets channel, and the event kinematics cannot be solved unambiguously.

To fully establish the event kinematics, the momenta of the two neutrinos have to be known. To solve for the neutrino momenta, we first correct the reconstructed jet energy to the parton level to extract the b-quark 4-vector, and finally solve the event kinematics.

\subsubsection{Particle Level Corrections}

The final state partons undergo fragmentation and hadronization forming jets of particles, whose response is measured by the calorimeter. Hence, the 4-vector of a jet is different from the 4-vector of the corresponding final state parton, and a correction is required to extract the parton's 4-vector. As the energy spectrum of jets originating from b-quarks is different from the energy spectrum of jets originating from light partons due to the different fragmentation and hadronization functions, different corrections were extracted for $\mathrm{b}$ and light jets.

For the full Run IIa dataset with the corresponding JES correction, these so-called particle level corrections (PLCs) were extracted using signal Monte Carlo in [105] following a method described in [106] on so-called lepton+jet events where one of the $\mathrm{W}$ bosons decays hadronically: $t \bar{t} \rightarrow b \bar{b}+\ell+\nu+j j$. Thus, particle level corrections for light jets from the $\mathrm{W}$ decay and b-jets can be derived.

However, only a sizable number of b-jets is expected in the dimuon final state as both $W$ bosons decay leptonically. Thus, we derived PLCs for b-jets only. First, jets were selected following the description in Sec. 6.3.1, but with the cut on jet $p_{T}$ lowered to 15 $\mathrm{GeV}$. In addition to the jet selection, the muons necessary to correct the jets containing semileptonic decays of heavy quarks were selected. Next, the selected jets were matched to b partons from top decays within a cone of radius $\Delta R(b-$ parton, $b-j e t)=0.5$ if and only if the reconstructed jet under consideration is isolated from any other reconstructed 
jet within a radius of $\Delta R($ jet, jet $)=1.0$, twice the jet reconstruction cone size. This is necessary to veto any occasional hard gluon emission of the b-quarks that would lead to an additional reconstructed jet. Thus, the matching of a b-quark to a single jet is no longer possible. Furthermore, the jet - parton match has to be unique. If more than one $\mathrm{b}$ parton can be matched to the reconstructed jet it is not taken into account for deriving the parton level corrections. Finally, two dimensional histograms were filled with the jet and parton energies in different $\eta$ ranges, ranging from zero to 1.5 in 0.5 steps and a last $\eta$ bin which contains all jets with $|\eta|>1.5$. The resulting histogram of $E_{\text {jet }}$ versus $E_{\text {parton }}$ in the $0<|\eta|<0.5$ bin is shown in Fig. 7.1. The final step consists of creating a histogram with the mean reconstructed jet energy for any $E_{\text {parton }}$ parton bin, and fitting a $2^{\text {nd }}$ order polynomial to it in the range $30 \mathrm{GeV}<E_{\text {parton }}<400 \mathrm{GeV}$. This histogram as well as the fitted function and the resulting parameters are shown in Fig. 7.2. The two dimensional and $\left\langle E_{\text {jet }}\right\rangle$ vs. $E_{\text {parton }}$ histograms for all $\eta$ bins are shown in Appendix B. Table 7.1 summarizes the fit parameters for the different $\eta$ bins.

Table 7.1.: Summary of the PLC parameters. The reconstructed jet energy $E_{\text {jet }}$ is parametrized as $E_{\text {jet }}=a+b \times E_{\text {parton }}+c \times E_{\text {parton }}^{2}$.

\begin{tabular}{l|l|l|l}
\hline \hline$\eta$ bin & $\mathrm{a} / \mathrm{GeV}$ & $\mathrm{b}$ & $\mathrm{c} / \mathrm{GeV}^{-1}$ \\
$0<|\eta|<0.5$ & $5.376 \pm 0.247$ & $8.348 \pm 0.046) \times 10^{-1}$ & $(6.762 \pm 0.191) \times 10^{-4}$ \\
$0.5<|\eta|<1.0$ & $6.92 \pm 0.35$ & $8.046 \pm 0.067) \times 10^{-1}$ & $(7.075 \pm 0.295) \times 10^{-4}$ \\
$1.0<|\eta|<1.5$ & $8.596 \pm 0.508$ & $7.686 \pm 0.083) \times 10^{-1}$ & $(6.501 \pm 0.31) \times 10^{-4}$ \\
$1.5<|\eta|$ & $19.68 \pm 0.7$ & $6.644 \pm 0.084) \times 10^{-1}$ & $(6.733 \pm 0.206) \times 10^{-4}$
\end{tabular}

To test the applicability of these particle level corrections to dilepton events, we compare the top quark mass calculated from generated b quark, charged lepton and neutrino momenta with the invariant mass calculated from the reconstructed $\mathrm{b}$ jet, the generated charged lepton and neutrino. Last, the reconstructed b jet energy is corrected with the PLC, and the b jet four vector scaled appropriately before calculating the top quark invariant mass. The reconstructed jets are JES corrected and jet smearing shifting and removal is applied (see Sec. 5.2.6). Events are selected as described in Sec. 6.3, with $p_{T}>20 \mathrm{GeV}$ for all jets. The results are shown in Fig. 7.3. Applying particle level corrections to the reconstructed b-jets leads to a slightly larger RMS of the top mass distribution. At the same time, the fitted mean of the peak of the distribution is in good agreement with the generated top mass of $m_{\text {top }}=170 \mathrm{GeV}$. 


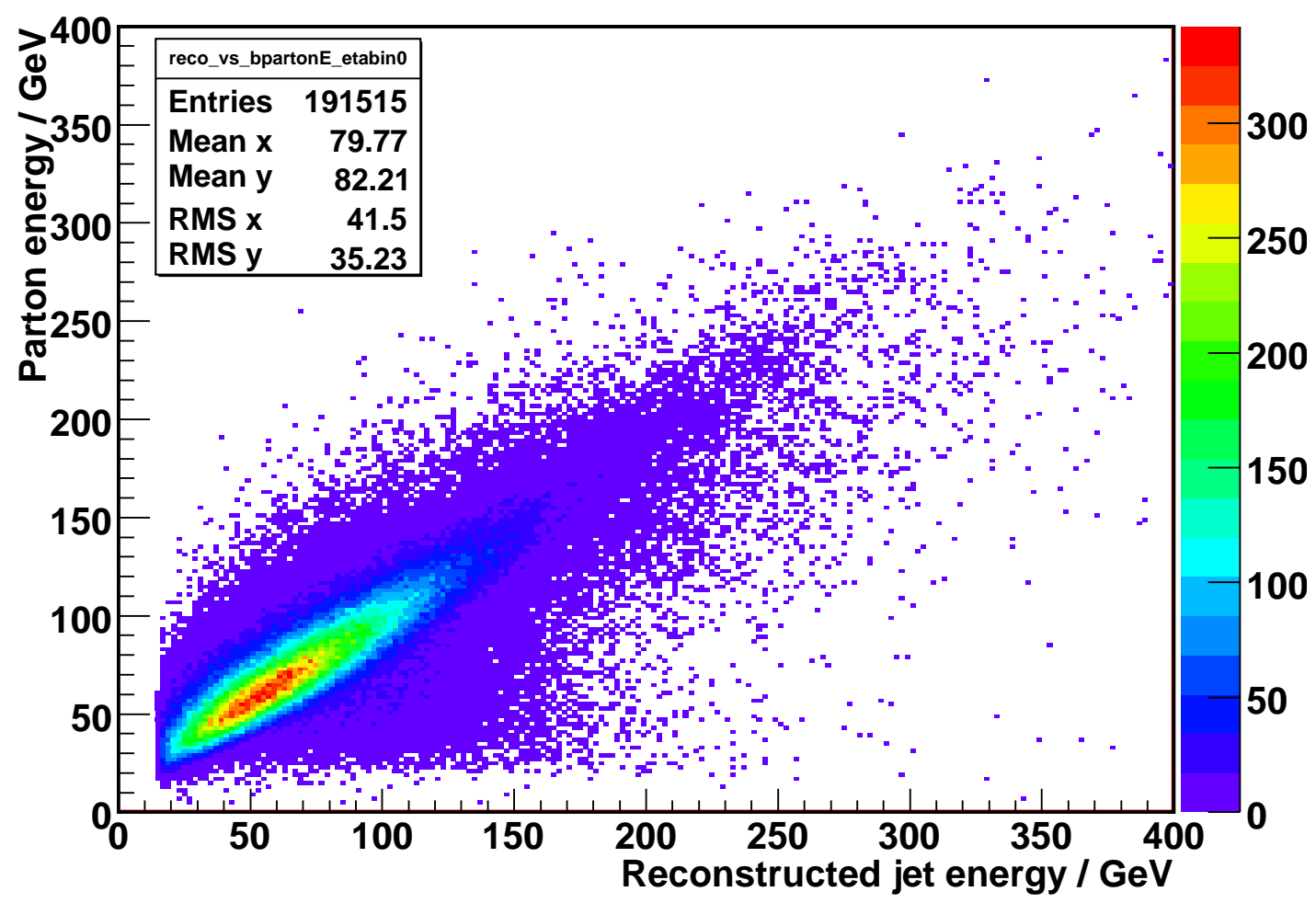

Figure 7.1.: $E_{\text {jet }}$ versus $E_{\text {parton }}$ in the $0<|\eta|<0.5$ bin.

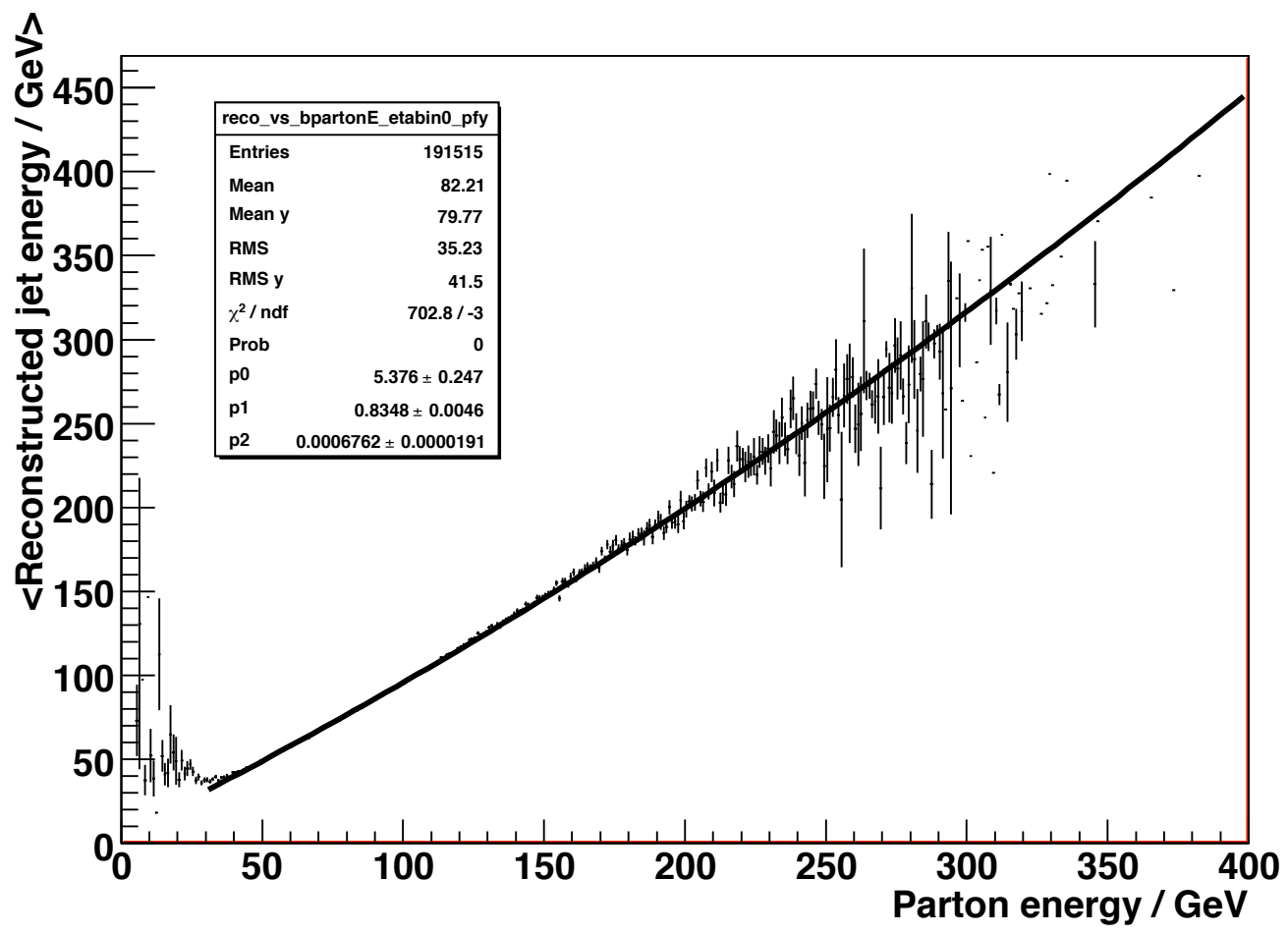

Figure 7.2.: $\left\langle E_{\text {jet }}\right\rangle$ vs. $E_{\text {parton }}$ histogram derived from Fig. 7.1. The solid line is the result of the fit in the shown region of $30 \mathrm{GeV}<\mathrm{E}_{\text {parton }}<400 \mathrm{GeV}$. 


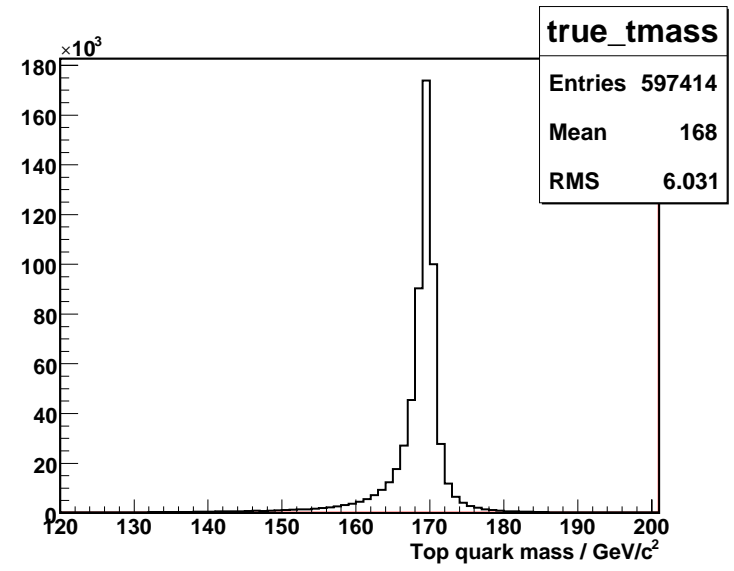

(a) Generated invariant top mass

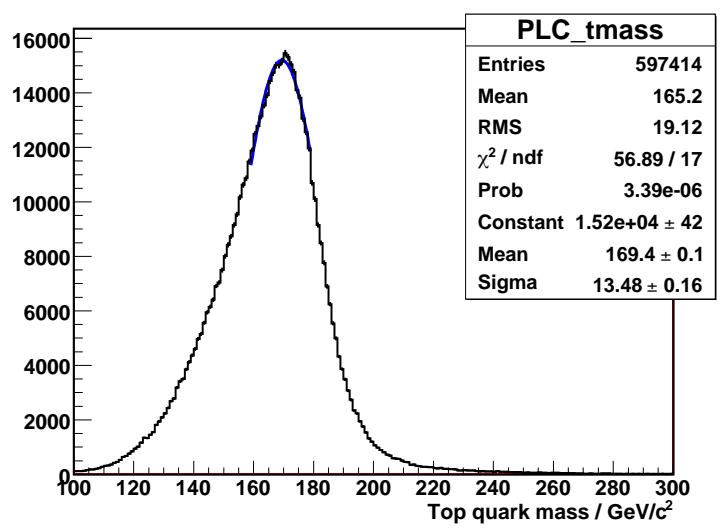

(c) Invariant top mass calculated from reconstructed and PLC corrected b-jets and the generated neutrino and muon

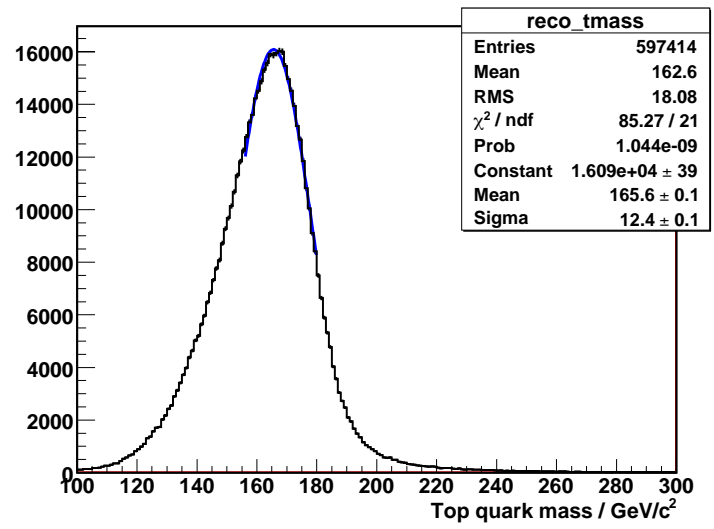

(b) Invariant top mass calculated from reconstructed b-jets and the generated neutrino and muon

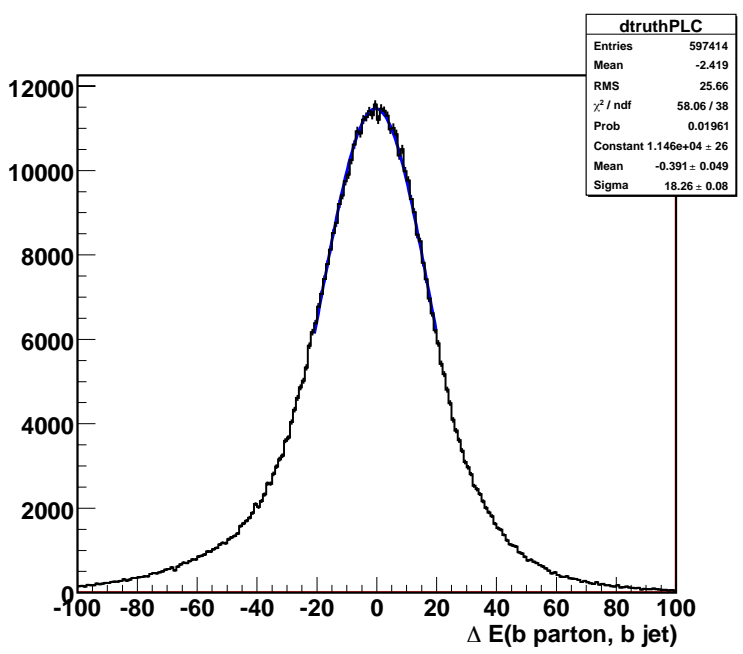

(d) Absolute difference in energy between the PLC corrected b jet and the generated b quark.

Figure 7.3.: Invariant top mass distributions using the generated b quark, the reconstructed b jet and the PLC corrected b jet. The Monte Carlo sample was generated with $m_{\text {top }}$ set to $170 \mathrm{GeV}$. 


\subsubsection{Solving the Event Kinematics}

The $t \bar{t}$ decay kinematics can be described by the following system of equations

$$
\begin{aligned}
E_{x} & =p_{\nu_{x}}+p_{\bar{\nu}_{x}} \\
E_{y} & =p_{\nu_{y}}+p_{\bar{\nu}_{y}} \\
E_{\nu}^{2} & =p_{\nu_{x}}^{2}+p_{\nu_{y}}^{2}+p_{\nu_{z}}^{2} \\
E_{\bar{\nu}}^{2} & =p_{\bar{\nu}_{x}}^{2}+p_{\bar{\nu}_{y}}^{2}+p_{\bar{\nu}_{z}}^{2} \\
m_{W^{+}}^{2} & =(\nu+\bar{\ell}) \cdot(\nu+\bar{\ell}) \\
& =\left(E_{\nu}+E_{\bar{\ell}}\right)^{2}-\left(p_{\nu_{x}}+p_{\bar{\ell}_{x}}\right)^{2}-\left(p_{\nu_{y}}+p_{\bar{\ell}_{y}}\right)^{2}-\left(p_{\nu_{z}}+p_{\bar{\ell}_{z}}\right)^{2} \\
m_{W^{-}}^{2} & =(\bar{\nu}+\ell) \cdot(\bar{\nu}+\ell) \\
m_{t}^{2} & =\left(W^{+}+b\right) \cdot\left(W^{+}+b\right) \\
& =(\nu+\bar{\ell}+b) \cdot(\nu+\bar{\ell}+b) \\
m_{\bar{t}}^{2} & =\left(W^{-}+\bar{b}\right) \cdot\left(W^{-}+\bar{b}\right) \\
& =(\bar{\nu}+\ell+\bar{b}) \cdot(\bar{\nu}+\ell+\bar{b})
\end{aligned}
$$

in a c.m. reference frame where the $\mathrm{z}$ axis coincides with the beam axis, and the $\mathrm{x}$ and $\mathrm{y}$ axis span the plane perpendicular to the beam axis. This system of equations can be solved for the neutrino momentum, resulting in a polynomial of order 4 for the neutrino $\mathrm{z}$ momentum. There is at most a fourfold ambiguity [107]. For each solution, the top quark and $\mathrm{W}$ boson 4-momenta are calculated. The first is needed to perform the boost to the top quark rest frame, while the latter provides an additional particle, besides the lepton and b-quark, available to calculate the spin correlation with.

The performance of the method can be described with four quantities. The ratio between the number of events that can be solved and all events given to the solver is called solution efficiency. The list of performance estimators is completed by the fraction of events with exactly two solutions of the number of events with at least one solution, the average number of solutions and the RMS.

These quantities have been calculated for a number of different scenarios, from making use of the full generator information to using the reconstructed objects and the pole masses for the top quarks and $W$ bosons. The results are shown in Tab. 7.2. Replacing the generated with the reconstructed muon ( (d) in Tab. 7.2) raises the ratio of events with exactly two solutions to events with at least one solution to $87 \%$ compared to the case where the generated 4-vectors and $\mathrm{W}$ boson and top quark pole masses are given to the solver ( (b) in Tab. 7.2), but the general solver efficiency drops by about $12 \%$.

Introducing the measured $E_{T}$ for the missing transverse energy calculated from generated neutrino momenta (e), the solver efficieny is decreased further, but at the same time the fraction of events with exactly two solutions is increased by $2 \%$. Once the generated $\mathrm{b}$ quark 4-momenta are replaced by the reconstructed and PLC corrected b-jets ( (f) in Tab. 7.2), the solver efficiency is further reduced.

Using the wrong, same charge sign lepton and b jet, paring leads to substantial decreased but not vanishing solver efficiency. As can be seen from the third to last and second to last row of Tab. 7.2, there is a substantial difference in solver efficiency between using both parton matched b-quark - lepton permutations and pairing the leptons with the leading and next-to-leading jet in the event. 
Up to this stage, both b-quarks are required to match a reconstructed jet, and the generated muons are required to match reconstructed muons. Dropping this requirement and therefor not performing any parton - reconstructed object matching, we arrive at the solver performance shown in the last row of Tab. 7.2. In the following, each event solved is weighted with $1 / \mathrm{N}, \mathrm{N}$ being the number of solutions. Events with no solutions are dropped.

Table 7.2.: The efficiency to find at least one solution, the ratio of events with exactly two solutions to the number of events with at least one solution, the average number and RMS of solutions when at least one solution was found for different input scenarios.

\begin{tabular}{l|l|l|l|l}
\hline \hline & Solution efficiency & $\frac{N_{\text {sol }}=2}{N_{\text {sol }}>0}$ & $\left\langle N_{\text {sol }}>0\right\rangle$ & $\mathrm{RMS}\left(N_{\text {sol }}>0\right)$ \\
\hline t, W masses known exactly (a) & $96.68 \%$ & 0.85 & 2.80 & 0.71 \\
\hline t, W pole mass assumed (b) & $86.15 \%$ & 0.83 & 2.78 & 0.68 \\
\hline $\begin{array}{l}\text { t, W pole mass assumed, } \\
\text { both } b \bar{b} \text { permutations (c) }\end{array}$ & $89.56 \%$ & 0.64 & 3.38 & 1.30 \\
\hline \hline Reconstructed leptons (d) & $74.64 \%$ & 0.87 & 2.76 & 0.67 \\
\hline Reconstructed $E_{T}(\mathrm{e})$ & $72.67 \%$ & 0.89 & 2.72 & 0.62 \\
\hline $\begin{array}{l}\text { Reconstructed b-jets } \\
\text { (parton matched, no PLC) }\end{array}$ & $61.09 \%$ & 0.87 & 2.75 & 0.67 \\
\hline $\begin{array}{l}\text { Reconstructed b-jets } \\
\text { (parton matched, PLC) (f) }\end{array}$ & $57.49 \%$ & 0.88 & 2.75 & 0.66 \\
\hline $\begin{array}{l}\text { Wrong b-jet permutation } \\
\text { (parton matched, PLC) }\end{array}$ & $26.37 \%$ & 0.90 & 2.70 & 0.60 \\
\hline $\begin{array}{l}\text { Both bb permutations } \\
\text { (parton matched) }\end{array}$ & $65.89 \%$ & 0.65 & 3.34 & 1.28 \\
\hline $\begin{array}{l}\text { Reconstructed b-jets } \\
\text { (using leading \& ntl jet, } \\
\text { both permutations) (g) }\end{array}$ & $50.14 \%$ & 0.68 & 3.26 & 1.26 \\
\hline \hline $\begin{array}{l}\text { Requiring no } \\
\text { parton - reconstructed } \\
\text { object matching } \\
\text { (both permutations) (h) }\end{array}$ & 56.58 & 0.65 & 3.34 & \\
\hline
\end{tabular}

\subsection{Reweighting the PYTHIA Monte Carlo}

The matrix element implemented in PYTHIA [72] 73] does average over all top and antitop quark spin directions before top and anti-top decay. Therefore, we have to reweigh the simulated events to reproduce a certain spin correlation strength C. An appropriate weight $w$ is the right hand side of Eq. 2.67,

$$
w=1+C \cos \theta_{\ell} \cos \theta_{\bar{\ell}}
$$

The angles $\theta_{\ell}$ and $\theta_{\bar{\ell}}$ are defined as follows. Starting with the top and anti-top 4-momenta in the laboratory frame, a rotation-free boost to the $t \bar{t}$ rest frame is performed. The 


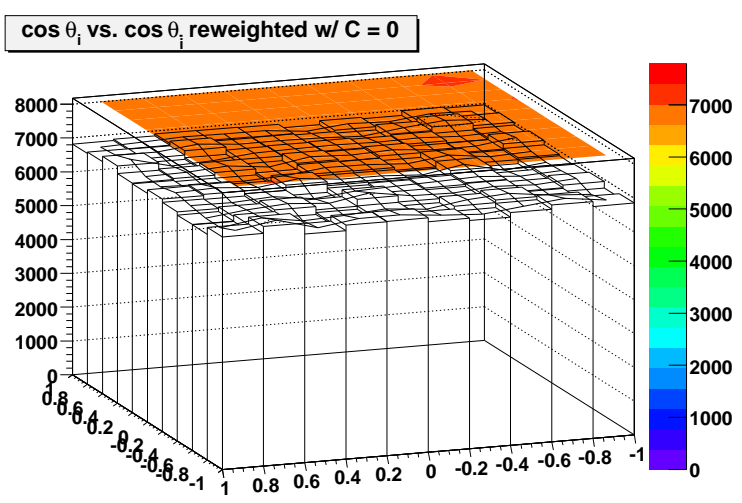

(a) Original $\cos \theta_{\ell}$ vs. $\cos \theta_{\bar{\ell}}$ distribution from PYTHIA

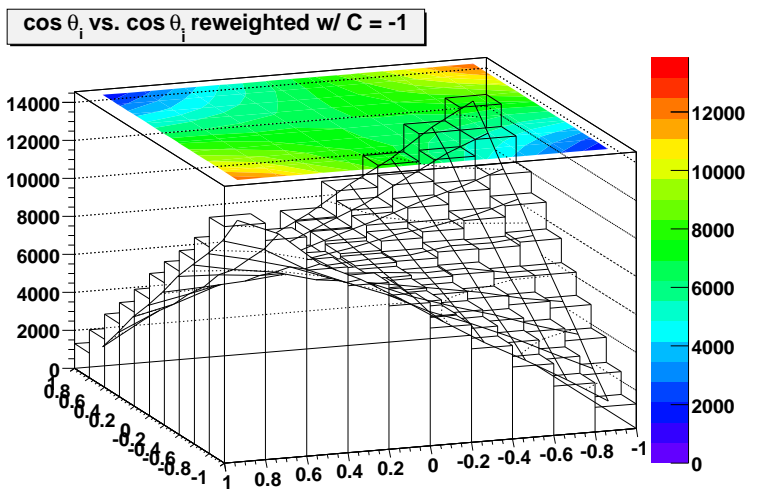

(c) $\cos \theta_{\ell}$ vs. $\cos \theta_{\bar{\ell}}$ distribution when reweighing PYTHIA Monte Carlo to $C_{\ell \bar{\ell}}=-1$

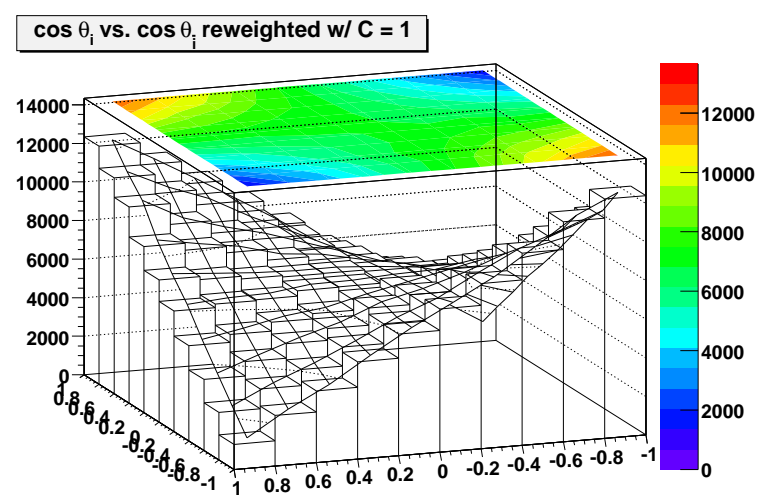

(b) $\cos \theta_{\ell}$ vs. $\cos \theta_{\bar{\ell}}$ distribution when reweighing PYTHIA Monte Carlo to $C_{\ell \bar{\ell}}=1$

Figure 7.4.: Examples of $2 \mathrm{D} \cos \theta_{\ell}$ vs. $\cos \theta_{\bar{\ell}}$ distributions .

direction of the proton beam, $\hat{p}$, is computed in that frame. Finally, a rotation-free boost is performed from the $t \bar{t}$ rest frame to the top (anti-top) rest frame, and the top (anti-top) quark decay product directions $\hat{q}_{1}\left(\hat{q_{2}}\right)$ are computed in that frame. Now $\cos \theta_{\bar{\ell}}=\hat{p} \cdot \hat{q_{1}}$ and $\cos \theta_{\ell}=\hat{p} \cdot \hat{q_{2}}$. The reweighting is performed at Monte Carlo truth level, i.e. with the 4-momenta generated by PYTHIA. Fig. 7.4 shows the initial and reweighed $\cos \theta_{\ell}$ vs. $\cos \theta_{\bar{l}}$ distributions.

\subsection{Asymmetry Measurement}

Using the notation of Sec. 2.4.1, we can identify $t_{\uparrow}$ with $\cos \theta>0$ and $t_{\downarrow}$ with $\cos \theta<0$ for any top decay product $(\ell, \mathrm{b}$, or $\mathrm{W})$. Here, $\theta$ is the angle between the top spin direction and the quantization axis, $0<\theta<\pi / 2$ for $t_{\uparrow}$ and $\pi / 2<\theta<\pi$ for $t_{\downarrow}$. For likesign spin top quark pairs, the product of the cosines is greater than zero, while for unlikesign spins the product is smaller than zero. Integrating the right hand side of Eq. 2.67 in these regions 
yields

$$
\begin{aligned}
\int_{0}^{1} \int_{0}^{1} \frac{1+C \cos \theta_{i} \cos \theta_{\bar{i}}}{4} d\left(\cos \theta_{i}\right) d\left(\cos \theta_{\bar{i}}\right) & =\frac{1}{4}+\frac{C}{16} \\
\int_{-1}^{0} \int_{-1}^{0} \frac{1+C \cos \theta_{i} \cos \theta_{\bar{i}}}{4} d\left(\cos \theta_{i}\right) d\left(\cos \theta_{\bar{i}}\right) & =\frac{1}{4}+\frac{C}{16} \\
\int_{-1}^{0} \int_{0}^{1} \frac{1+C \cos \theta_{i} \cos \theta_{\bar{i}}}{4} d\left(\cos \theta_{i}\right) d\left(\cos \theta_{\bar{i}}\right) & =\frac{1}{4}-\frac{C}{16} \\
\int_{0}^{1} \int_{-1}^{0} \frac{1+C \cos \theta_{i} \cos \theta_{\bar{i}}}{4} d\left(\cos \theta_{i}\right) d\left(\cos \theta_{\bar{i}}\right) & =\frac{1}{4}-\frac{C}{16}
\end{aligned}
$$

where the first two integrals refer to likesign spin top pairs, and the latter to integrals represent unlikesign spin top pairs. Now,

$$
\begin{gathered}
\int_{0}^{1} \int_{0}^{1} \frac{1+C \cos \theta_{i} \cos \theta_{\bar{i}}}{4} d\left(\cos \theta_{i}\right) d\left(\cos \theta_{\bar{i}}\right)+\int_{-1}^{0} \int_{-1}^{0} \frac{1+C \cos \theta_{i} \cos \theta_{\bar{i}}}{4} d\left(\cos \theta_{i}\right) d\left(\cos \theta_{\bar{i}}\right)- \\
\int_{-1}^{0} \int_{0}^{1} \frac{1+C \cos \theta_{i} \cos \theta_{\bar{i}}}{4} d\left(\cos \theta_{i}\right) d\left(\cos \theta_{\bar{i}}\right)-\int_{0}^{1} \int_{-1}^{0} \frac{1+C \cos \theta_{i} \cos \theta_{\bar{i}}}{4} d\left(\cos \theta_{i}\right) d\left(\cos \theta_{\bar{i}}\right)=\frac{C}{4}
\end{gathered}
$$

This can be simplified to

$$
\begin{gathered}
\int_{0}^{1} \frac{1+C \cos \theta_{i} \cos \theta_{\bar{i}}}{4} d\left(\cos \theta_{i} \cos \theta_{\bar{i}}\right)- \\
\int_{-1}^{0} \frac{1+C \cos \theta_{i} \cos \theta_{\bar{i}}}{4} d\left(\cos \theta_{i} \cos \theta_{\bar{i}}\right)=\frac{C}{4}
\end{gathered}
$$

which is the basis of the asymmetry measurement. The coefficient $\mathrm{C}$ depends on the spin analyzing powers $\alpha_{i}$ and $\alpha_{\bar{i}}$ of the decay products of the top quarks chosen to measure the spins with. Using both lepton and anti-lepton, measuring $\cos \theta_{\ell}$ and $\cos \theta_{\bar{\ell}}$, one determines $C_{\ell \bar{\ell}}$ etc. In measuring the asymmetry by using leptons, b-quarks and $W^{ \pm} \mathrm{s}$ from (anti-)top decays, it is possible to deduce the spin analyzing powers $\alpha_{i}$ s and the spin asymmetry trough Eqns. and .

An example for the distributions $\cos \theta_{i} \times \cos \theta_{\bar{i}}$ at $\mathrm{MC}$ truth level is shown in Fig. 7.5. Here, $i(\bar{i})$ can be either $\ell(\bar{\ell}), \bar{b}(b)$ or $W^{-}\left(W^{+}\right)$. The events have been reweighed as described in Sec. 7.2 to describe a spin correlation strength of one. Measuring the asymmetry in these plots as described above leads to $\left.C\right|_{\text {meas }}$. These measurements can be repeated for different values of the correlation coefficient $\left.C\right|_{\text {gen }}$ used to reweigh the events, and it is possible to determine calibration lines and thus correct the measured 


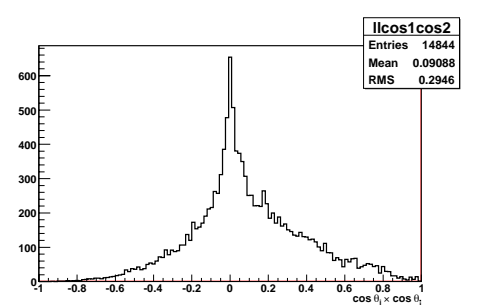

(a) $\cos \theta_{\ell} \times \cos \theta_{\bar{\ell}}$

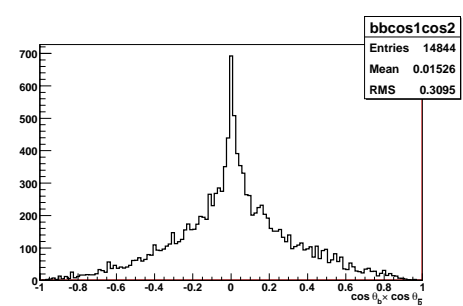

(d) $\cos \theta_{b} \times \cos \theta_{\bar{b}}$

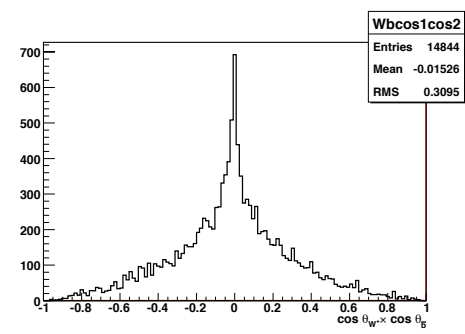

(g) $\cos \theta_{W^{+}} \times \cos \theta_{\bar{b}}$

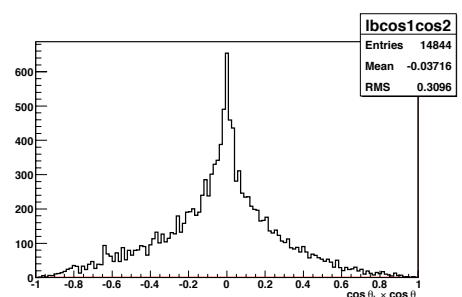

(b) $\cos \theta_{b} \times \cos \theta_{\ell}$

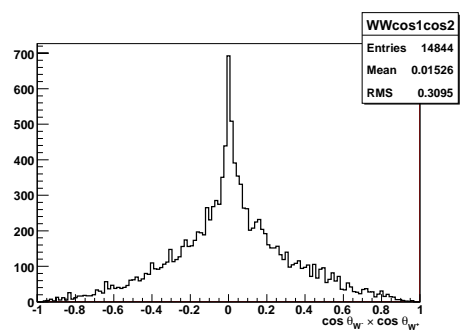

(e) $\cos \theta_{W^{+}} \times \cos \theta_{W^{-}}$

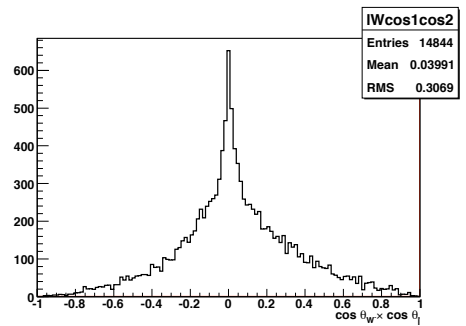

(h) $\cos \theta_{W-} \times \cos \theta_{\bar{\ell}}$

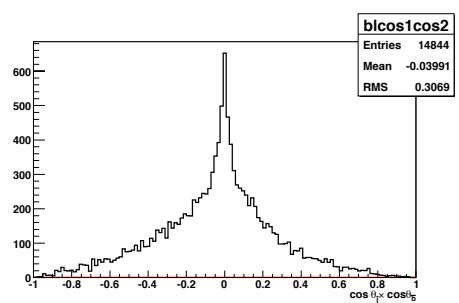

(c) $\cos \theta_{\bar{\ell}} \times \cos \theta_{\bar{b}}$

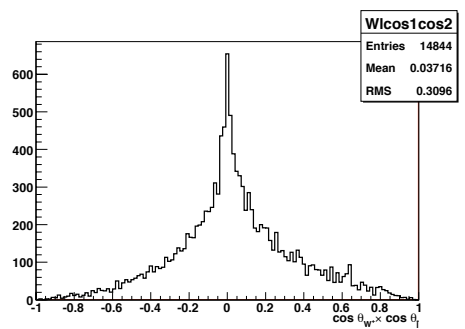

(f) $\cos \theta_{W^{+}} \times \cos \theta_{\ell}$

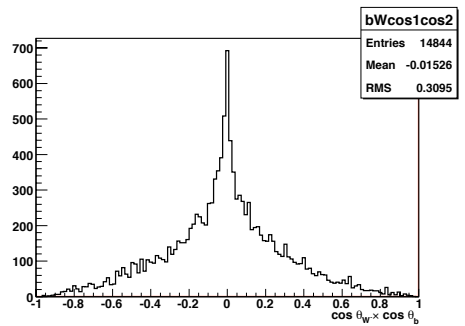

(i) $\cos \theta_{W}-\times \cos \theta_{b}$

Figure 7.5.: Various $\cos \theta_{i} \times \cos \theta_{\bar{i}}$ distributions when $C_{\ell \bar{\ell}}$ is reweighed to one. Similar to the reweighting procedure, generated 4-momenta were employed to create the distributions.

spin correlation for any offsets that might have been introduced by our method. Fig. 7.6 shows the calibration curves for $C_{i \bar{i}}$ as measured with the generated 4-vectors, where $i(\bar{i})$ is again either $\ell(\bar{\ell})$ or $b(\bar{b})$. The measured spin correlation strengths follow the reweighted correlation, although the slope is not equal to one for the $C_{\ell \bar{\ell}}$ calibration curve. This can be attributed to the event selection which cuts out regions of phase space thus distorting the asymmetry distribution. Similarly, the slope of the other calibration curves is different from the predicted $\alpha_{i} \alpha_{\bar{i}}$ value.

Next, we examine the effect of final state parton showering, event reconstruction and event selection on the asymmetry measurement through the calibration curves of the asymmetry in the $\cos \theta_{i} \times \cos \theta_{i}$ distribution. We compare different settings in an analogous manner to Sec. 7.1 .2 and show the results for the $\cos \theta_{\ell} \times \cos \theta_{\bar{\ell}}$ and $\cos \theta_{b} \times \cos \theta_{\bar{b}}$ distributions in Figs. 7.7 and 7.8. The other lepton, b jet and reconstructed $\mathrm{W}$ pairings are shown in Appendix C.1. Comparing the top left-hand plots of $\left.C_{i \bar{i}}\right|_{\text {meas }}$ vs. $\left.C_{i \bar{i}}\right|_{\text {gen }}$ with the calibration curves of the measurement at MC truth level, one notices that the slopes of the former are less dependent on the final state particles chosen to measure the spin correlation than the latter. Thus, it seems important to measure the spin correlation not only for the 


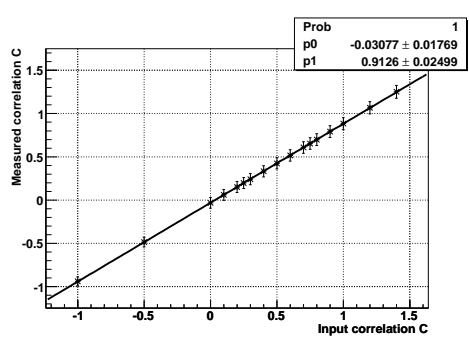

(a) $\cos \theta_{\ell} \times \cos \theta_{\bar{\ell}}$

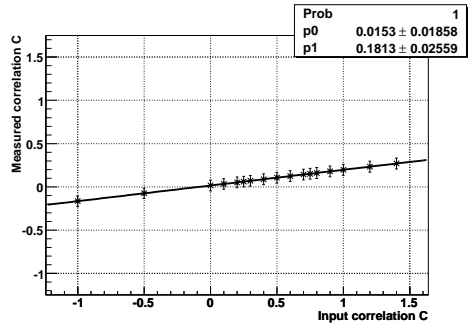

(d) $\cos \theta_{b} \times \cos \theta_{\bar{b}}$

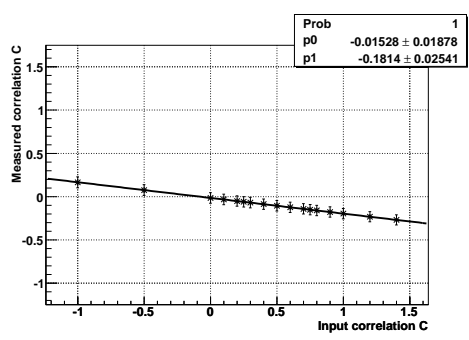

(g) $\cos \theta_{W^{+}} \times \cos \theta_{\bar{b}}$

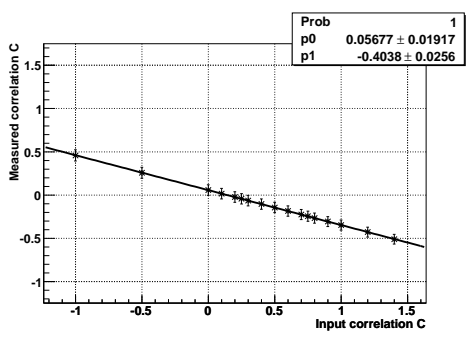

(b) $\cos \theta_{b} \times \cos \theta_{\ell}$

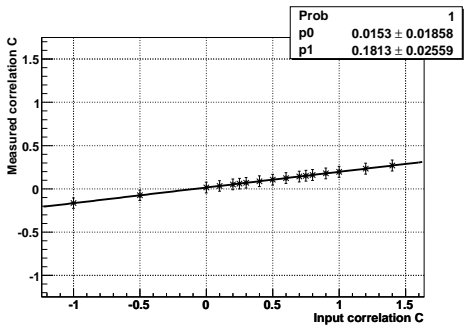

(e) $\cos \theta_{W^{+}} \times \cos \theta_{W^{-}}$

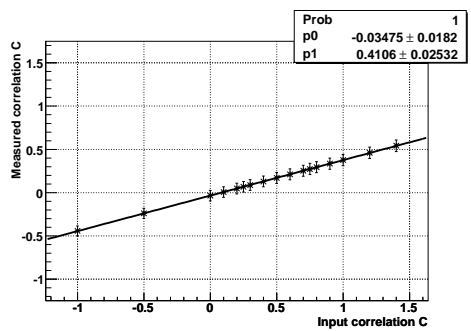

(h) $\cos \theta_{W^{-}} \times \cos \theta_{\bar{\ell}}$

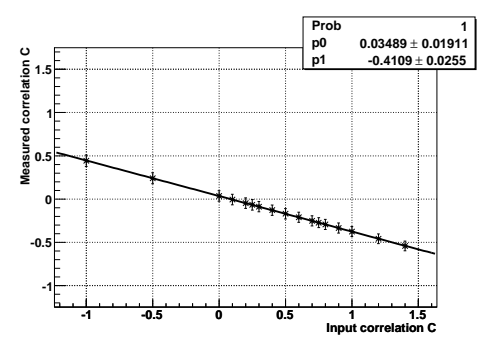

(c) $\cos \theta_{\bar{\ell}} \times \cos \theta_{\bar{b}}$

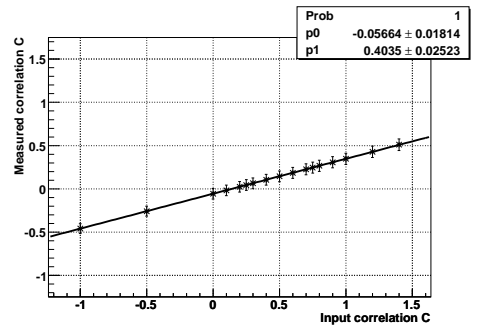

(f) $\cos \theta_{W^{+}} \times \cos \theta_{\ell}$

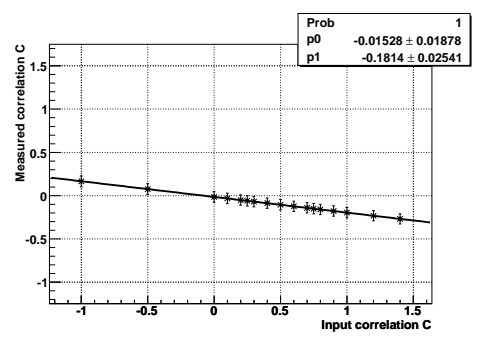

(i) $\cos \theta_{W}-\times \cos \theta_{b}$

Figure 7.6.: Calibration curves derived by measuring the spin asymmetry at MC truth level for various $\left.C_{\ell \bar{\ell}}\right|_{\text {gen }}$ values.

lepton- antilepton pair, but every paring of lepton, b-jet and $W$ boson available. Though the detector and additional jet effects are considerably smaller than the dilution induced by kinematic solving, the slope of spin correlation measurements involving leptons is affected most by these.

So far, all generated Monte Carlo events that pass the event selection have been used for these calibration plots. To examine the sensitivity of the measurement with the available data statistics and the expected number of signal events, ensemble tests have been performed. Alternatively, the sensitivity can be estimated using the slope $\mathrm{S}$ of the calibration curve and the RMS of the $\cos \theta_{i} \times \cos \theta_{\bar{i}}$ distribution,

$$
\text { Sensitivity }=\frac{S}{R M S_{\left.C\right|_{\text {meas }}}\left(C_{\text {gen }}=0\right)}
$$

Using the offset of the measurement, $\left.C\right|_{\text {meas }}$ at $\left(\left.C\right|_{\text {gen }}=0\right)$, and the measured correlation at $\left.C\right|_{\text {gen }}=1$, this can be expressed as

$$
\text { Sensitivity }=\frac{\left.C\right|_{\text {meas }}\left(\left.C\right|_{\text {gen }}=1\right)-\left.C\right|_{\text {meas }}\left(\left.C\right|_{\text {gen }}=0\right)}{R M S_{\left.C\right|_{\text {meas }}}\left(\left.C\right|_{\text {gen }}=0\right)}
$$


For ensemble testing, random events have been drawn from the pool of all selected signal events according to the distribution of $C_{\text {meas }}$ under consideration. The ensemble size was fixed to the number of expected signal events. $N^{\text {ensembles }}=100$ ensembles per calibration point and top - anti top quark decay product pairing were generated. In addition to the calibration curves, the mean and width of the pull

$$
\text { pull }=\frac{\left\langle\left. C^{i}\right|_{\text {meas }}-\left.C^{\text {all ensembles }}\right|_{\text {meas }}\right\rangle}{\sigma_{\left.C^{\text {all ensembles }}\right|_{\text {meas }}}}
$$

defined as

$$
\langle p u l l\rangle=\frac{1}{N} \sum_{i=1}^{N^{\text {ensembles }}} \frac{\left\langle\left. C^{i}\right|_{\text {meas }}-\left.C^{\text {all ensembles }}\right|_{\text {meas }}\right\rangle}{\left.\sigma_{C^{\text {all ensembles }}}\right|_{\text {meas }}}
$$

and

$$
\sigma_{\text {pull }}=\frac{1}{N^{2}} \sqrt{\sum_{i=1}^{N^{\text {ensembles }}}\left(\langle p u l l\rangle-\text { pull }^{i}\right)^{2}}
$$

have been determined to verify the ensemble tests. Here, $\mathrm{N}$ is the number of ensembles generated, $\left.C^{i}\right|_{\text {meas }}$ is the measured spin correlation in the $i$ th ensemble and pull $l^{i}$ is the pull value of the $i$ th ensemble. Finally, $\left.C^{\text {all ensembles }}\right|_{\text {meas }}$ and $\left.\sigma_{C^{\text {all ensembles }}}\right|_{\text {meas }}$ are the mean and width of the measured correlation distribution of all ensembles, respectively. Deviations of $\langle p u l l\rangle$ from zero and of $\sigma_{\text {pull }}$ from one would indicate an incorrect estimation of the errors derived by ensemble testing. As can be seen from Figs. 7.9 and 7.10 , this is not the case. As can be seen from Figs. 7.9 and 7.10 , the error on each individual point on the calibration curve is on the order of $\delta_{C}=1.5$. In Run I, the sensitivity of the asymmetry measurement was estimated to be on the order of 0.7 , which translates to an error of $\delta_{C} \approx$ 2. However, this analysis used the $e e, e \mu$ and $\mu \mu$ final states.

With a $\delta_{C}$ of 1.5, a measurement of the top-antitop spin correlation is not feasible with an integrated luminosity of $1 \mathrm{fb}^{-} 1$ in the dimuon final state only. One has either to include other final states or use the already available Run IIb data (or both). With the already recorded $4 \mathrm{fb}^{-} 1$, the statistical error would be reduced to $1 / 2$ of its current value. Including the dielectron and electron-muon final states in addition gives about four times the number of events, reducing the statistical error again by 0.5 . Thus, reducing the statistical uncertainty by a factor of $1 / 4$ is possible, which in turn renders a measurement of the spin correlation possible. 


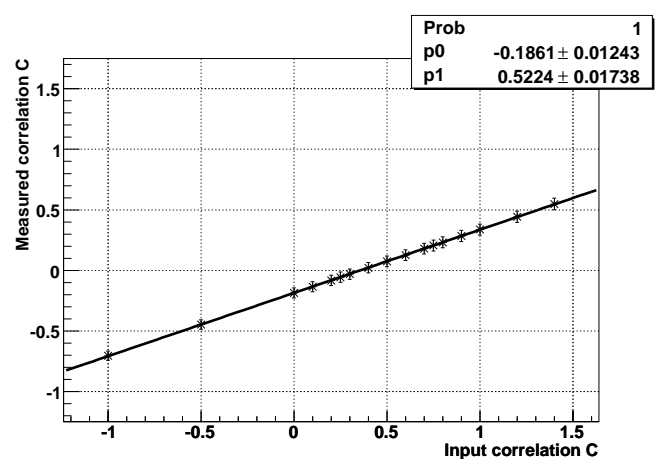

(a) t, W masses known exactly

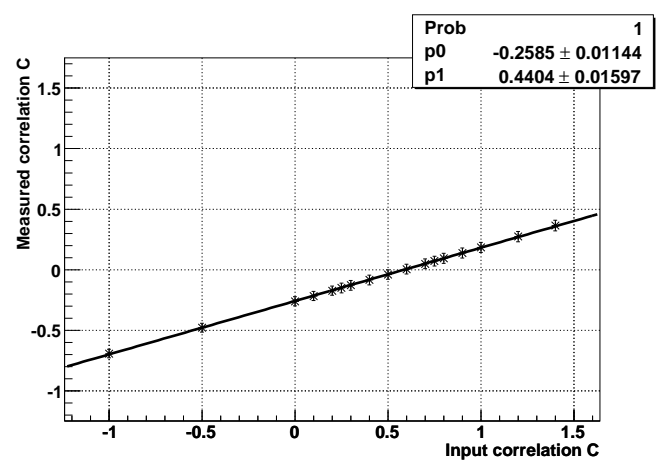

(c) t, W pole mass assumed, both $b \bar{b}$ permutations

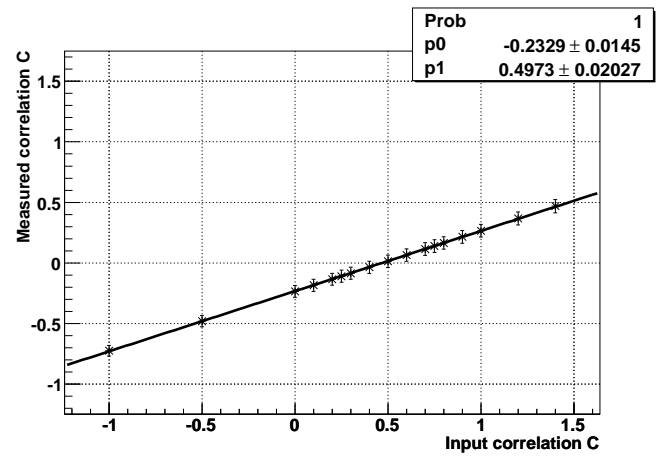

(e) Reconstructed $\not_{T}$

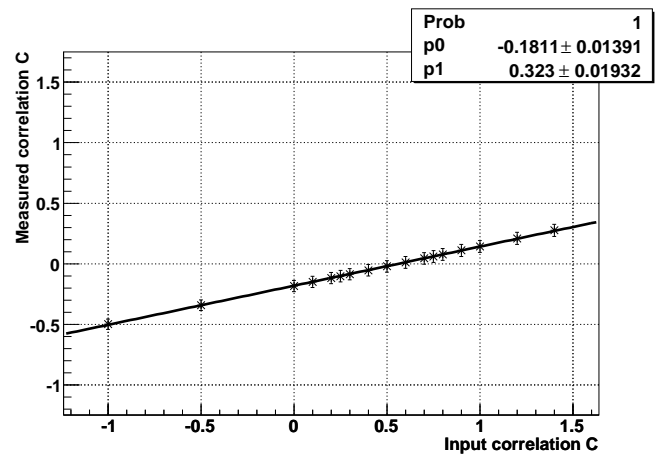

(g) Reconstructed b-jets (using leading \& ntl jet, both permutations)

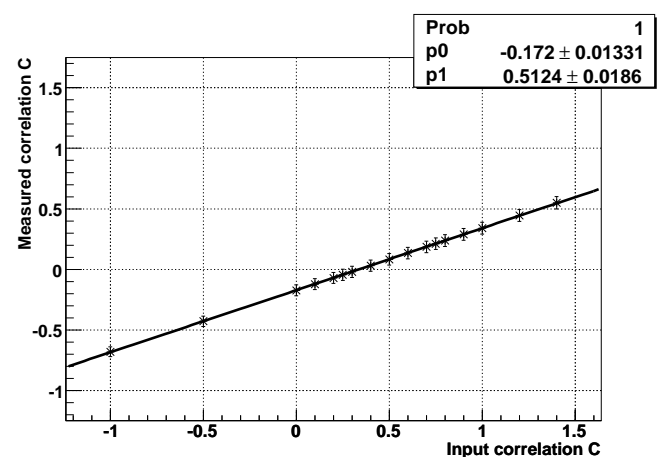

(b) t, W pole mass assumed

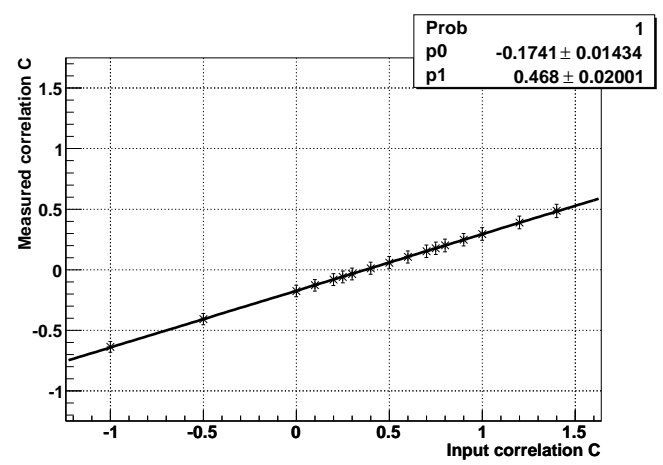

(d) Reconstructed leptons

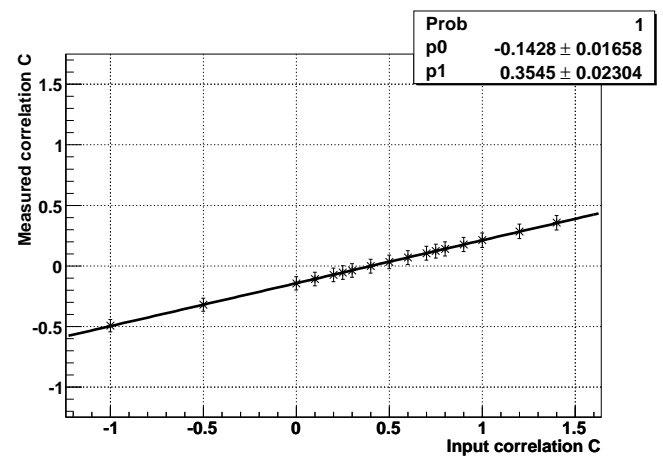

(f) Reconstructed b-jets (parton matched, PLC)

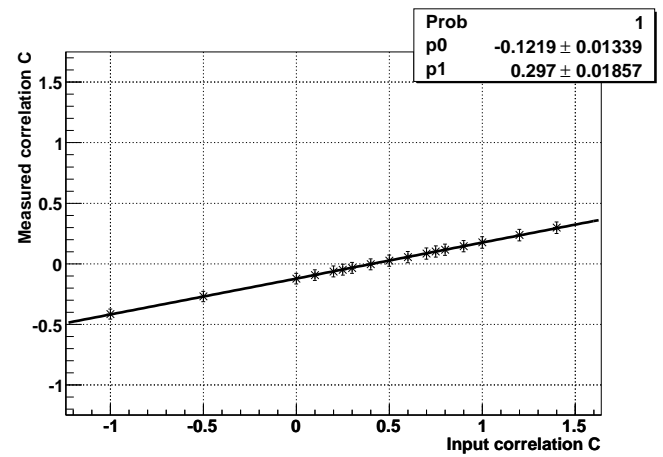

(h) No parton - reconstructed object matching required (both permutations)

Figure 7.7.: Calibration plots for the measurement of $C_{\ell \bar{\ell}}$. The letters correspond to Tab. 7.2 indicating the type of inputs to the kinematic solver. (h) uses no MC truth information and hence resembles the measurement in data. 


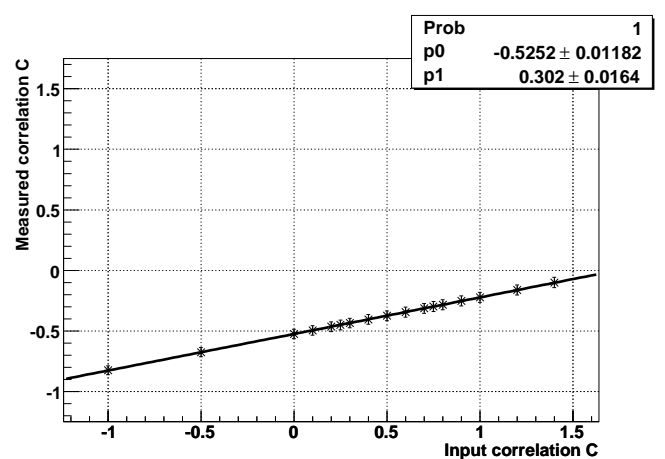

(a) t, W masses known exactly

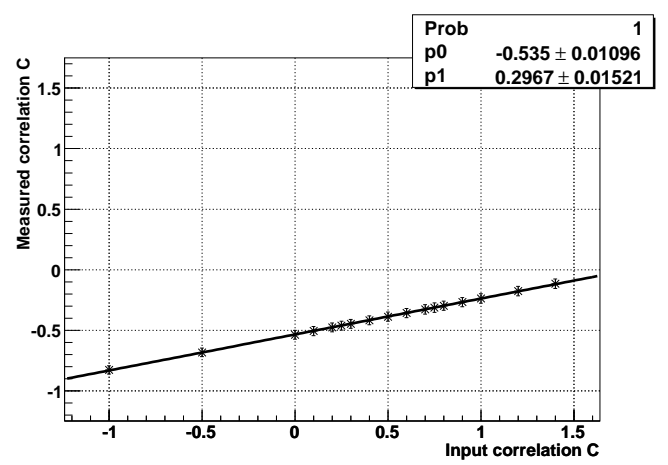

(c) t, W pole mass assumed, both $b \bar{b}$ permutations

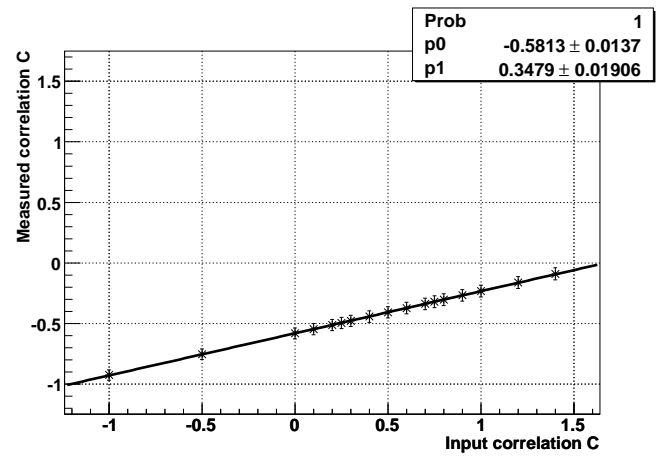

(e) Reconstructed $\not_{T}$

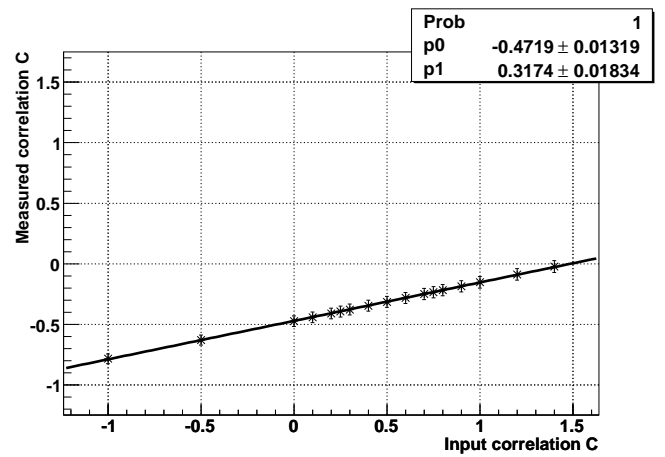

(g) Reconstructed b-jets (using leading \& ntl jet, both permutations)

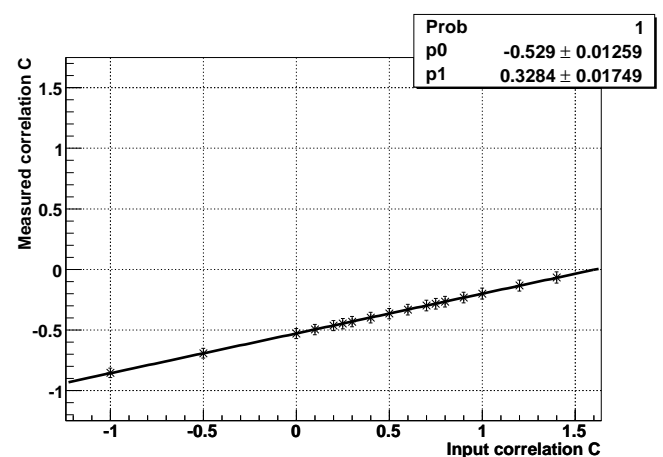

(b) t, W pole mass assumed

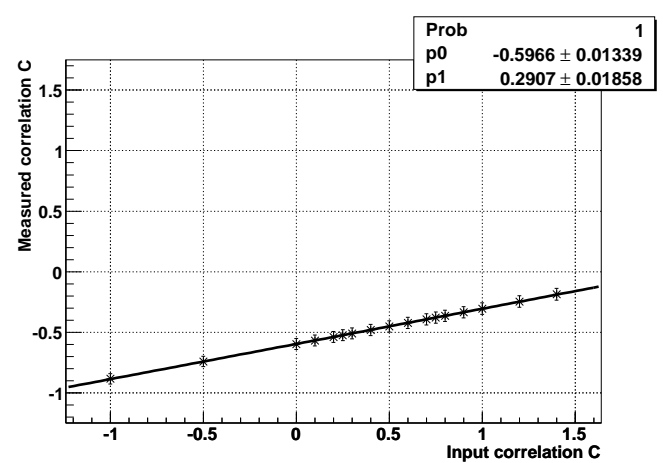

(d) Reconstructed leptons

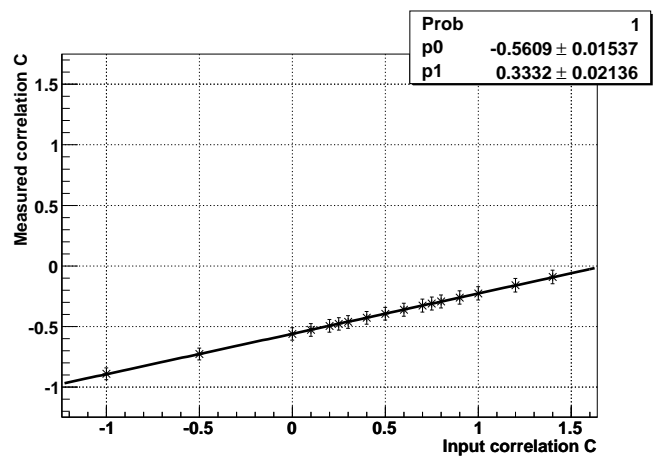

(f) Reconstructed b-jets (parton matched, PLC)

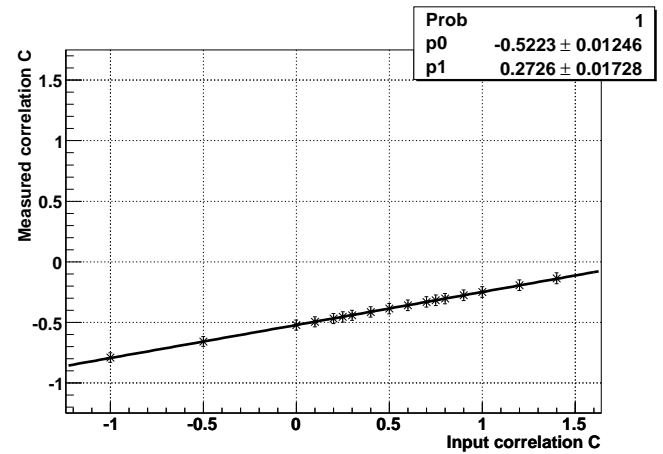

(h) No parton - reconstructed object matching required (both permutations)

Figure 7.8: Calibration plots for the measurement of $C_{b \bar{b}}$. The letters correspond to Tab. 7.2 indicating the type of inputs to the kinematic solver. (h) uses no MC truth information and hence resembles the measurement in data. 


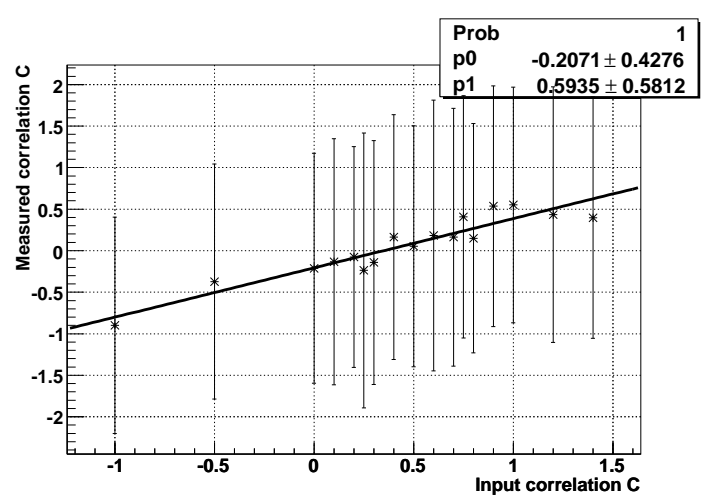

(a) t, W masses known exactly

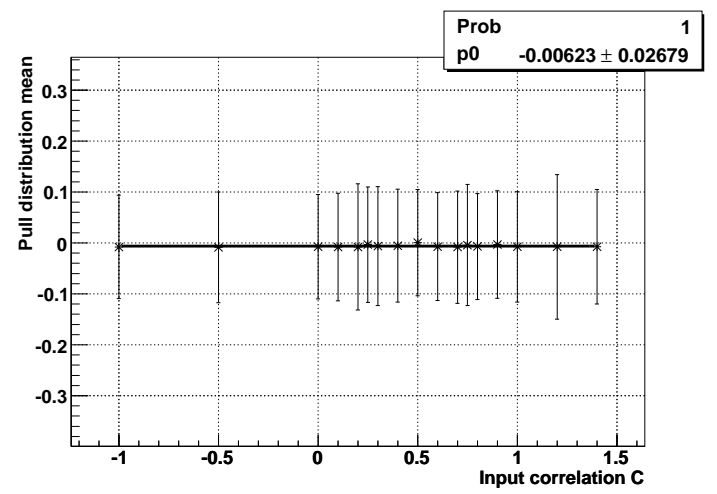

(c) Mean of the pull distribution for (b)

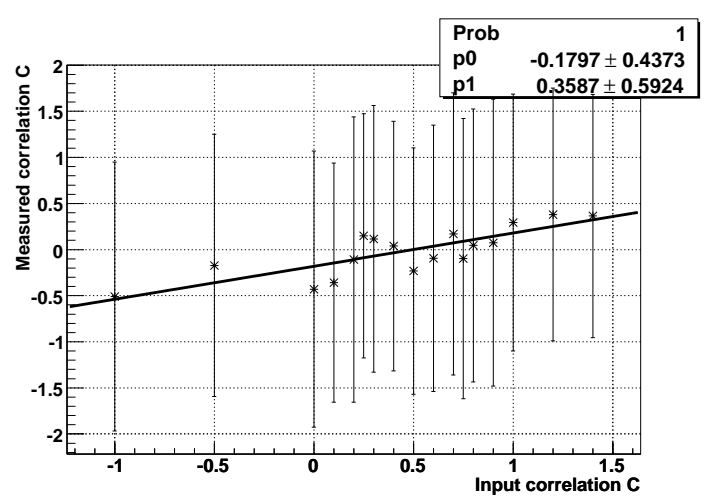

(b) Not parton - reconstructed object matching (both permutations)

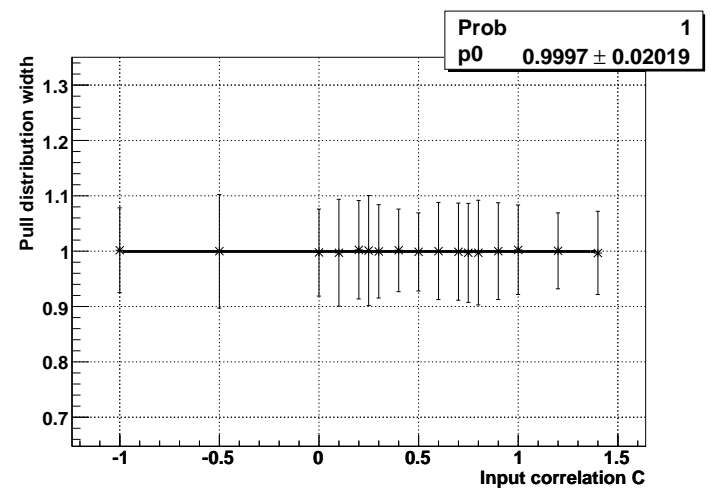

(d) Width of the pull distribution for (b)

Figure 7.9.: Ensemble tests for the measurement of $C_{\ell \bar{\ell}}$. 100 ensembles with 8 events each were generated. The error bars in the calibration curve plots indicate $\sigma_{C_{\text {meas }}^{\text {all ensembles }}}$ 


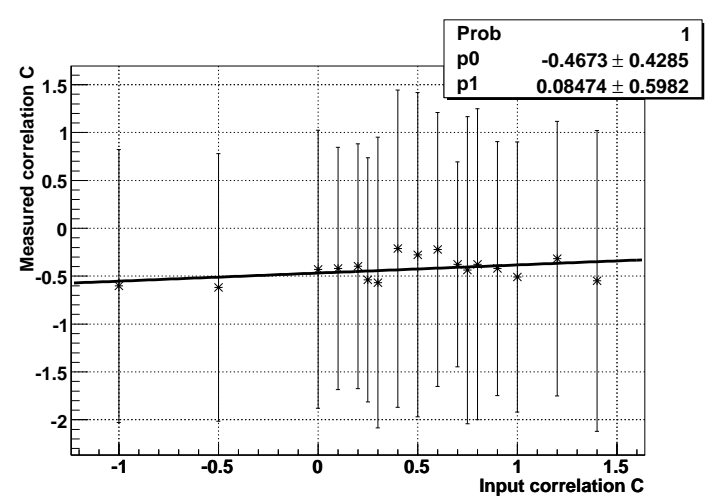

(a) t, W masses known exactly

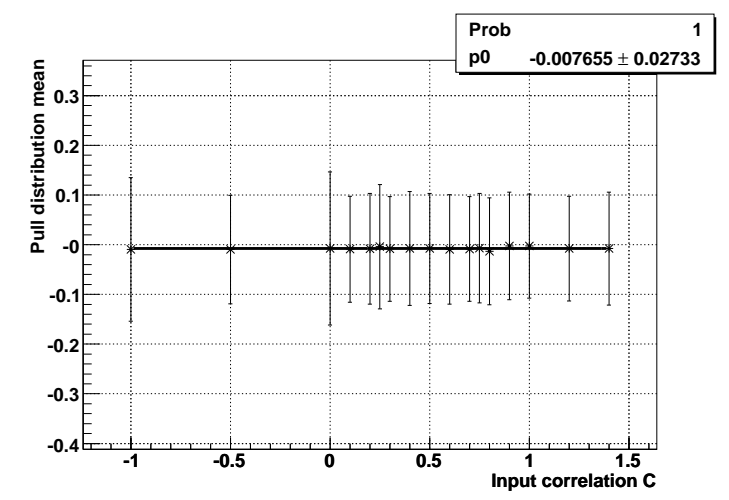

(c) Mean of the pull distribution for (b)

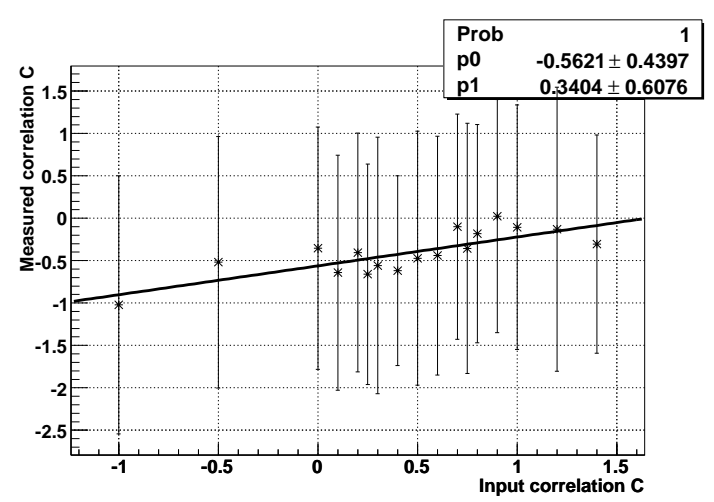

(b) Not parton - reconstructed object matching (both permutations)

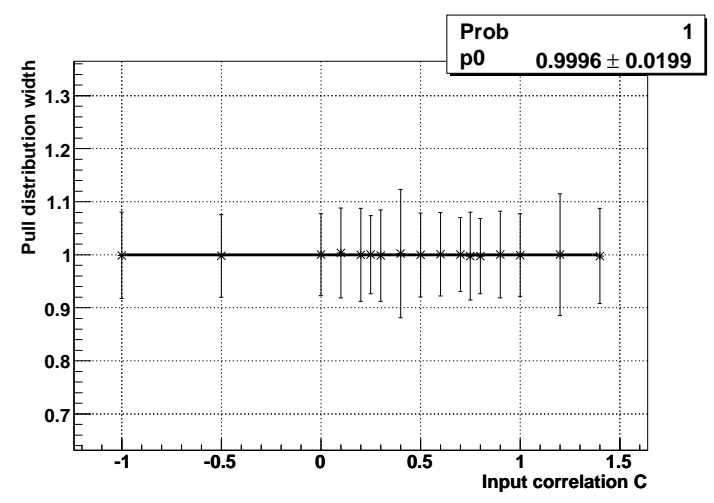

(d) Width of the pull distribution for (b)

Figure 7.10.: Ensemble tests for the measurement of $C_{b \bar{b}}$. 100 ensembles with 8 events each were generated. The error bars in the calibration curve plots indicate $\left.\sigma_{C^{a l l ~ e n s e m b l e s ~}}\right|_{\text {meas }}$ 
CHAPTER 7. MONTE CARLO STUDIES OF THE TOP - ANTI-TOP SPIN CORRELATION IN THE DIMUON FINAL STATE 


\section{Conclusion}

This thesis present: 1 a measurement of the $t \bar{t}$ production cross-section in the dimuon final state. The measurement utilizes the complete Run IIa dataset with an integrated luminosity of $1 \mathrm{fb}^{-1}$ that was recorded between June 2002 and February 2006. A series of cuts facilitating object and event selection was introduced to separate the $t \bar{t} \rightarrow b \bar{b}+\mu \bar{\mu}+E_{T}$ signature from the various backgrounds. The cuts have been optimized to reject $Z \rightarrow$ $\mu \bar{\mu}$ events, which is the most prominent background to this final state. To achieve a high rejection rate, the $\mathbb{E}_{T}$ significance variable has been introduced which is a powerful discriminator between events with missing transverse energy from neutrinos and $E_{T}$ from detector effects. Applying the event selection to the data set and carefully evaluating the backgrounds using simulated events and data, a cross section of

$$
\sigma_{t \bar{t} \rightarrow b \bar{b}+\mu \bar{\mu}+E_{T}}=5.08_{-2.83}^{+3.43} \text { (stat) }{ }_{-1.14}^{+1.31} \text { (syst) }{ }_{-0.61}^{+0.69} \text { (lumi) pb. }
$$

is measured. The systematic uncertainty is driven mainly by the uncertainty on the jet energy scale, the muon trigger efficiencies and the Monte Carlo statistics of the $\mathrm{Z} \gamma^{*}$ background. This cross section measurement is consistent with the most recent theoretical predictions. Having selected $t \bar{t}$ events from the Run IIa dataset recorded with the DØ detector is the basis for measuring the properties of the top quark such as mass, charge and spin correlations. Combining the cross section measurement presented in this thesis with the cross section measurements in other dilepton final states yields

$$
\text { dilepton : } \quad \sigma_{t t}=7.4_{-1.1}^{+1.2}(\text { stat })_{-0.9}^{+1.0} \text { (syst) } \mathrm{pb},
$$

Comparing the most recent theoretical predictions with the combined cross section, values for the top quark pole mass have been extracted, leading to

$$
m_{\text {top }}=172.80_{-8.38}^{+9.37}(\text { stat }+ \text { syst })_{-5.67}^{+4.47}(\text { theory }) \mathrm{GeV}(\mathrm{CTEQ} 6.5)
$$

and

$$
m_{\text {top }}=174.52_{-8.09}^{+9.03}(\text { stat }+ \text { syst })_{-4.37}^{+3.05}(\text { theory }) \mathrm{GeV}(\mathrm{MRST} 2006 \mathrm{NNLO})
$$

using the NLO+NLL predictions of Cacciari et. al. [46] and

$$
m_{\text {top }}=174.68_{-8.37}^{+9.25}(\text { stat }+ \text { syst })_{-4.29}^{+2.77}(\text { theory }) \mathrm{GeV}(\text { CTEQ6.5) }
$$

and

$$
m_{\text {top }}=176.17_{-8.08}^{+8.97}(\text { stat }+ \text { syst })_{-3.02}^{+1.58}(\text { theory }) \mathrm{GeV}(\mathrm{MRST} 2006 \mathrm{NNLO})
$$

\footnotetext{
${ }^{1}$ Preliminary results of this analysis have been reported in [1, [2], 3], 4], [5], [6], [7]. The final crosssection publication is in preparation.
} 
using the approximate NNLO prediction of Moch and Uwer [43]. As described in Sec. 6.5.2, these results are complementary to the mass measurements carried out using event kinematics. They have larger uncertainties associated with them as the latter due to the statistical and systematic uncertainty on the cross-section measurements in the dilepton final states.

As already indicated, the events selected by the cross section analyses are input to further measurements of top quark properties. One recent example is the search for charged Higgs bosons in the top quark decay chain. Selecting events from charged Higgs Monte Carlo samples with the existing dimuon, dielectron and electron-muon event selections yields the selection efficiency for $t \bar{t} \rightarrow H^{+} H^{-} b \bar{b}$ and $t \bar{t} \rightarrow W H b \bar{b}$. A maximum likelihood fit to the number of events observed in data was performed to extract the branching ratio of $t \rightarrow H^{+} b$. As no deviation from the Standard Model was observed, limits were set depending on the charged Higgs mass in the tauonic model [7], see Fig. 6.30. Including the lepton+jets final state to the charged Higgs search, the $t \bar{t}$ cross-section and $\operatorname{BR}\left(t \rightarrow H^{+} b\right)$ can be fit simultaneously. In addition, limits can be set for leptophobically decaying charged Higgs, as shown in Fig. 6.33 .

Spin correlations are another example of top quark properties measurements made possible by the growing statistics. Compared to the measurements carried out in Run I, that yielded a limit on the spin correlation $C_{\ell \bar{\ell}}$ of -0.25 at $95 \% \mathrm{CL}$, new methods were introduced to solve the kinematics of dileptonic $t \bar{t}$ decays, resulting in no, two or four neutrino momentum solutions per b quark - lepton permutation. A crucial input to this kinematic solver is the b parton momentum. So-called particle level corrections ( $P L C s$ ) have been derived to infer the b-parton momentum from the b-jet momentum by comparing b-jets and the quarks they originate from in Monte Carlo samples. Finally, the top quark pair spin correlation has been studied using not only the muon and anti-muon from the top quark pair decay, but including the b jets (corrected with PLCs) and the W bosons for both the top and anti-top. With ensemble tests, the sensitivity of the measurement was estimated. They clearly indicate the need to include final states other than the dimuon channel and to add the still growing Run IIb data set. 


\section{Acknowledgements}

On these last pages, I would like to thank the numerous people who made this thesis possible through their guidance and support.

First of all, I would like to thank Gregor Herten for giving me the opportunity to graduate in his group and his support during my time as a PhD student. Thank you very much for giving me the freedom to work with $\mathrm{D} \varnothing$ and always asking the right questions.

I am grateful to Sascha Caron for his patience and valuable help, ever since he came to Freiburg.

I am deeply indebted to Ursula Bassler and Christian "Beat in!" Schwanenberger for all their support, helpful discussions and guidance throughout the past years, especially during the hard time of conference rushes. Thanks for your advice and answering my countless questions.

I would like to continue with a big "thank you" to my D $\varnothing$ collegues which helped me with the analysis in various ways. First of all, Ulla and Carsten for sharing their wisdom and office space with an absolute newbie. Ingo, for the small and big problems we mastered and for making me laugh. It's a pitty you fled to the third floor. Henrik, for always keeping the right perspective. The "German Ghetto" at DØ made every stay at Fermilab a memorable experience. Thank you so much for your company and support! Yvonne and Alexander, I'm grateful for the (non-)physics discussions (Skype is great!), and cheering me up when things were going haywire.

Dan, for being a partner-in-crime not only at FNAL. Always eager for a coffee break, I enjoyed many not quite physics discussions with my fellow ATLAS collegues in Freiburg. Always prepared to rescue me, I'm in Florian and Asen's debt for their help with computing. Stephan, for the music. Up the irons! For useful physics advice, encouragement and fruitful discussions I am grateful to Lisa Shabalina, Frederic Deliot and Bertrand Martin dit Latour.

Once more I'd like to thank Ursula Bassler, Christian Schwanenberger, Sascha Caron and Ingo Torchiani for proof-reading this manuscript.

Zuletzt möchte ich mich ganz herzlich bei meiner Familie bedanken: bei meiner Frau Mahela für ihre Unterstützung und Geduld mit mir, ohne immer zu wissen, womit ich beschäftigt war, und meinen Töchtern, die mich mit der Physik teilen mußten. Besonderer Dank gebührt meinen Eltern, die mich meinen Weg gehen ließen und mich stets dabei unterstützt haben. Danke Euch allen für Eure Liebe und Euer Vertrauen! 
A. Data - Monte Carlo Comparison in
the Zero and Two Jet Inclusive Bin 


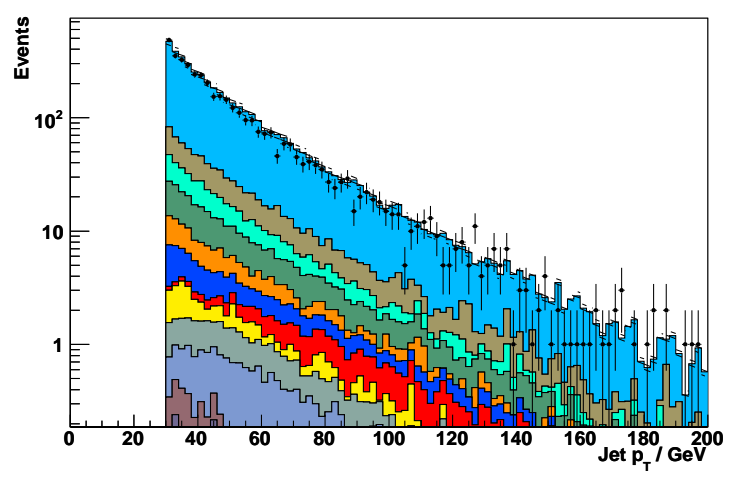

(a) 0 jet selection

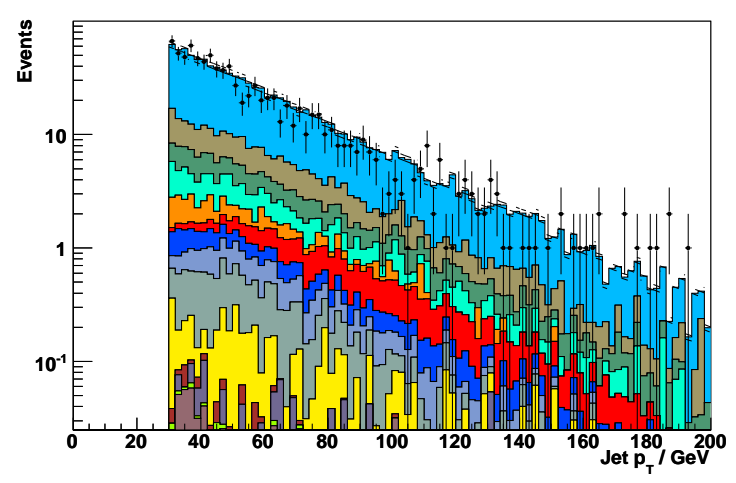

(b) 2 jet selection

Figure A.1.: Leading jet transverse momentum distribution.

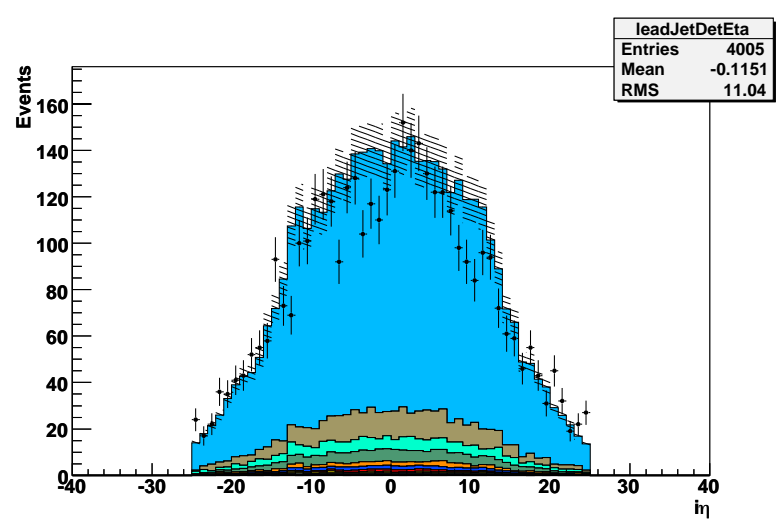

(a) 0 jet selection

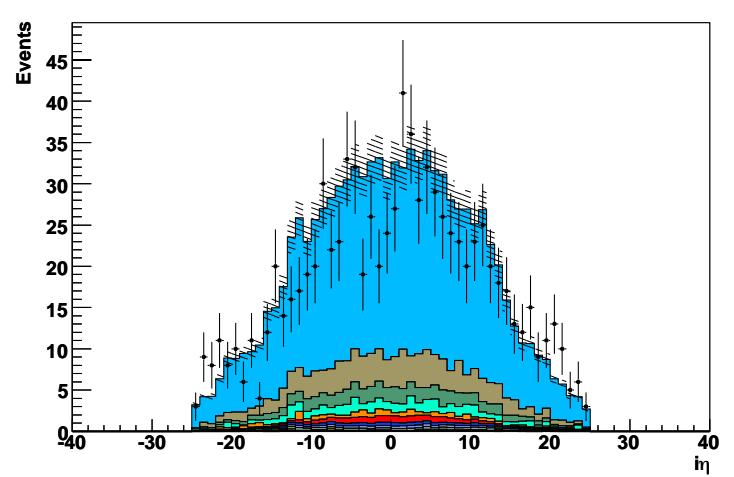

(b) 2 jet selection

Figure A.2.: Leading jet pseudo-rapidity distribution.

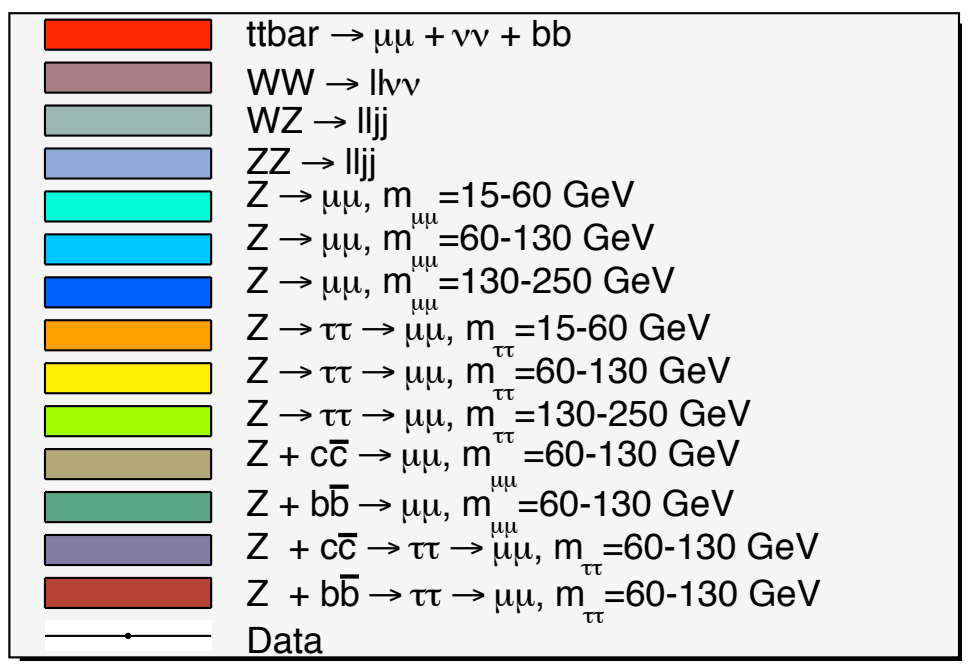

Figure A.3.: Color code used in the plots shown on this page 


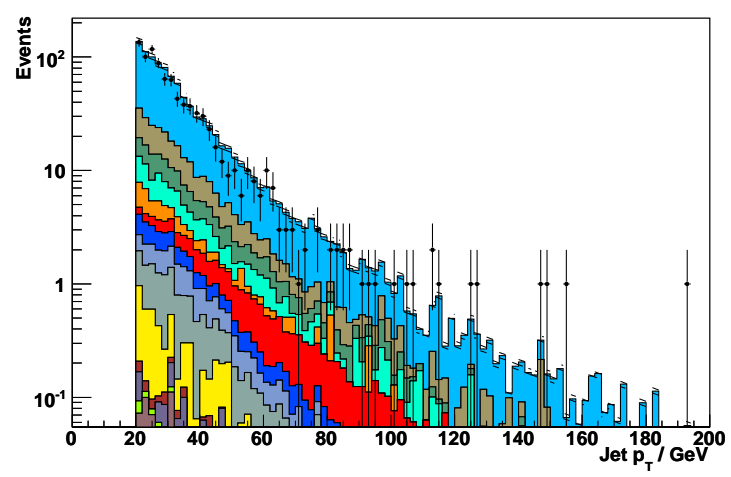

(a) 0 jet selection

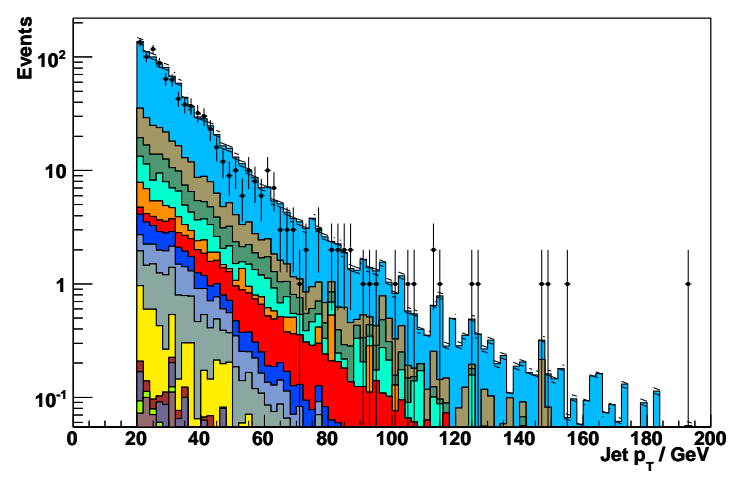

(b) 2 jet selection

Figure A.4.: Next to leading jet transverse momentum distribution.

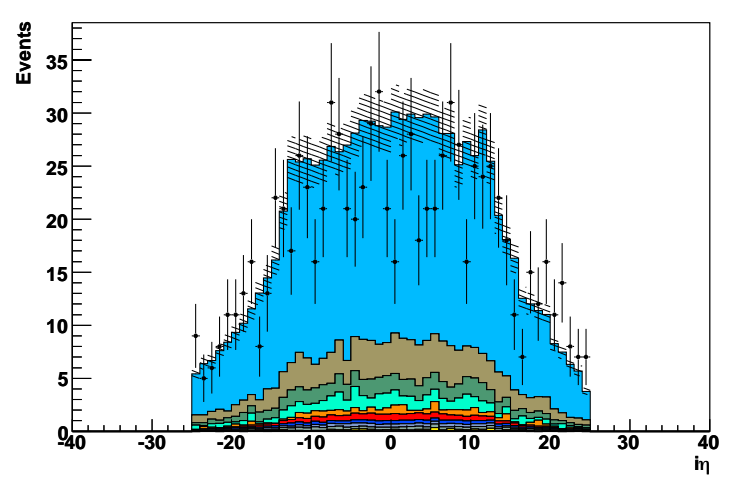

(a) 0 jet selection

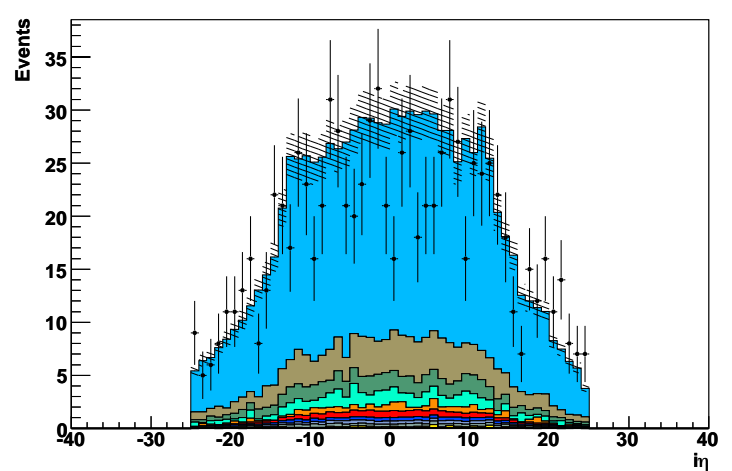

(b) 2 jet selection

Figure A.5.: Next to leading jet pseudo-rapidity distribution.

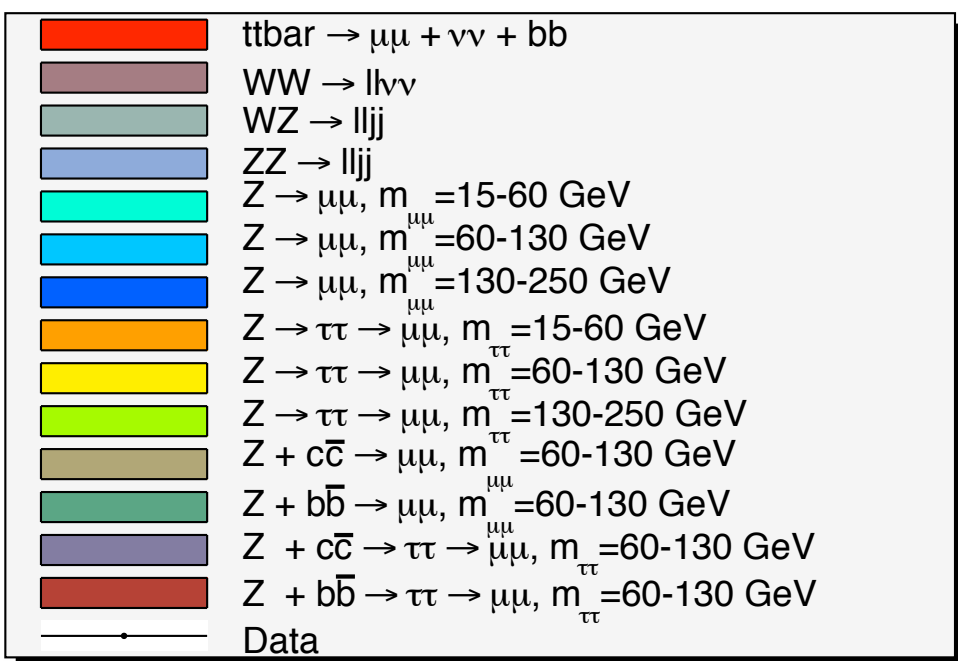

Figure A.6.: Color code used in the plots shown on this page 


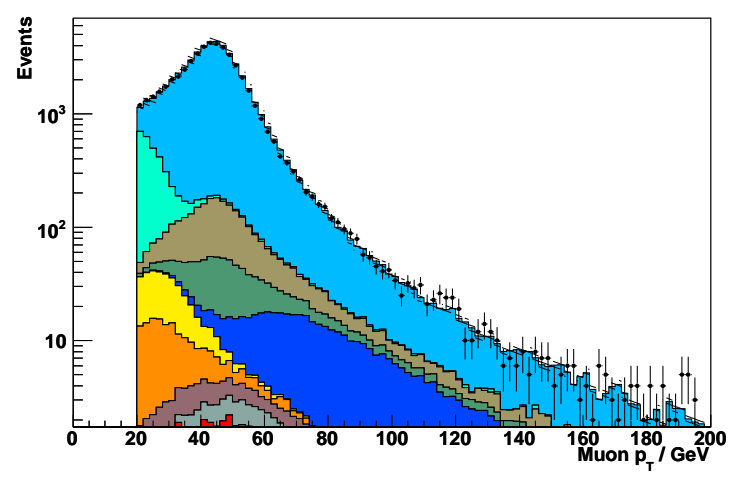

(a) 0 jet selection

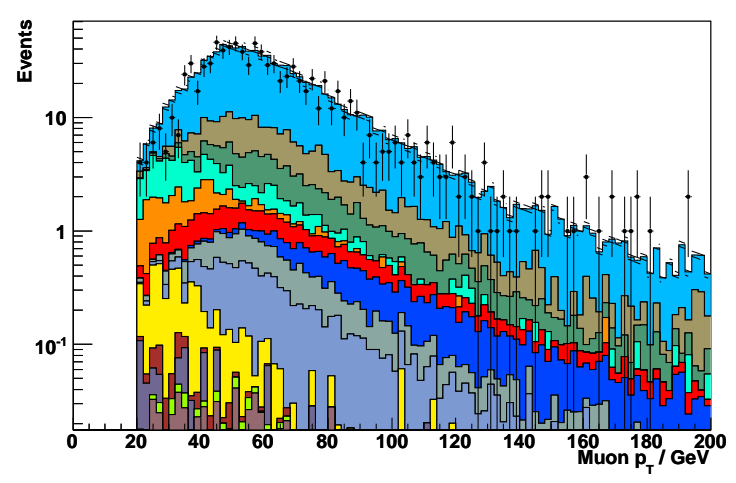

(b) 2 jet selection

Figure A.7.: Leading muon transverse momentum distributions.

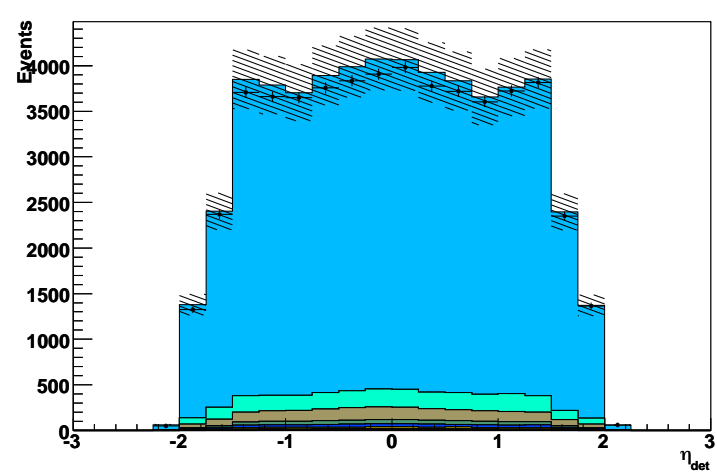

(a) 0 jet selection

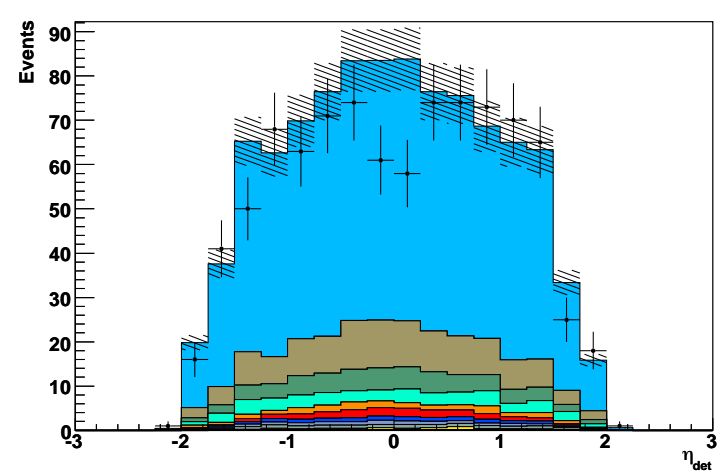

(b) 2 jet selection

Figure A.8.: Leading muon $\eta$ distributions.

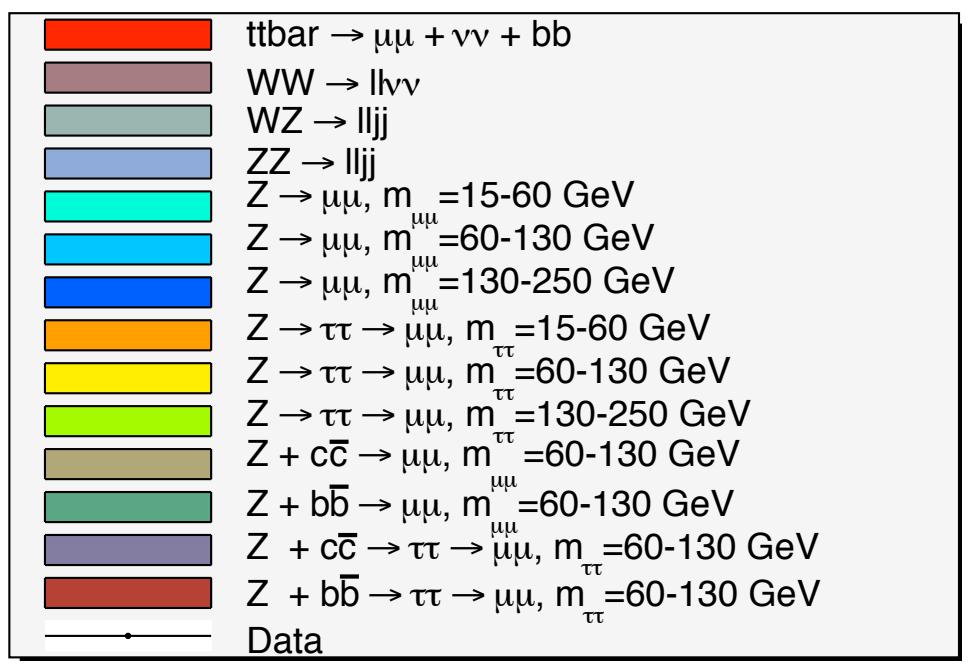

Figure A.9.: Color code used in the plots shown on this page 


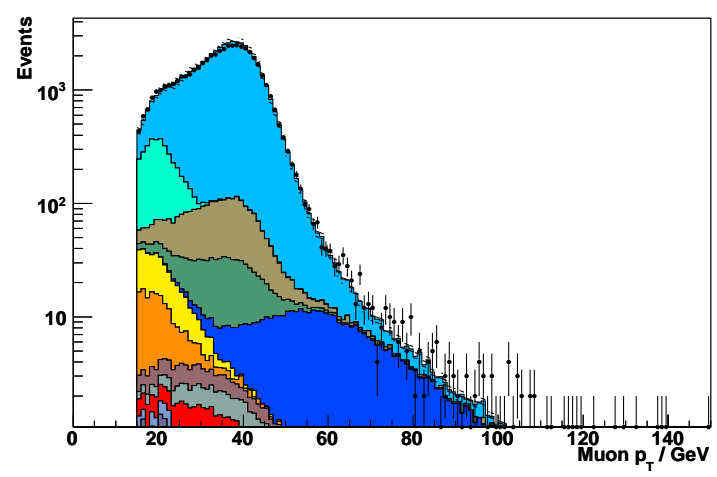

(a) 0 jet selection

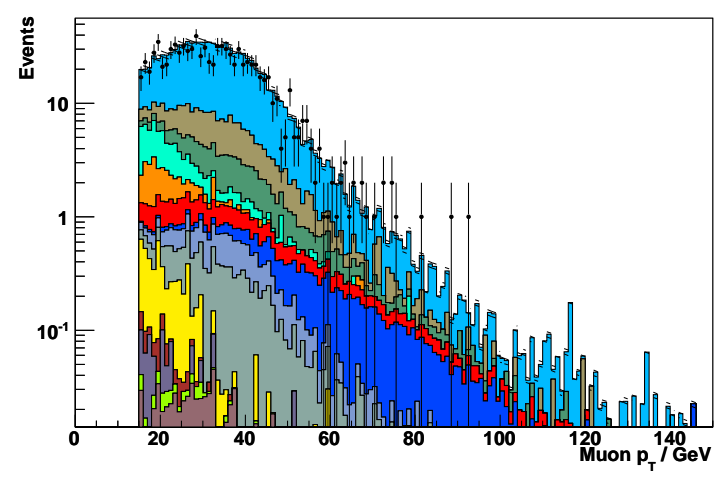

(b) 2 jet selection

Figure A.10.: Next to leading muon transverse momentum distributions.

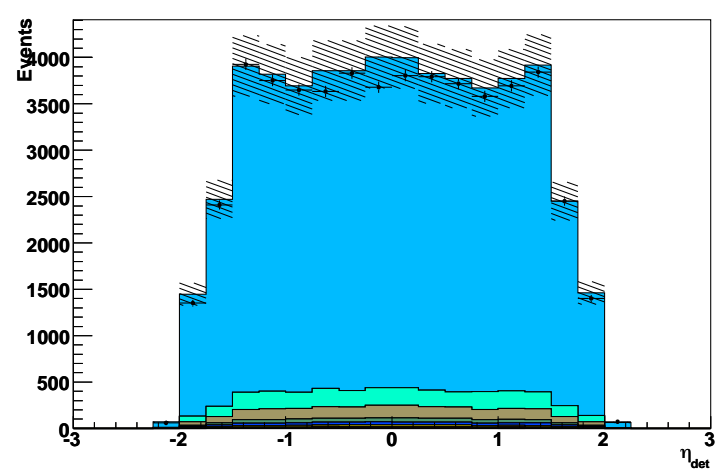

(a) 0 jet selection

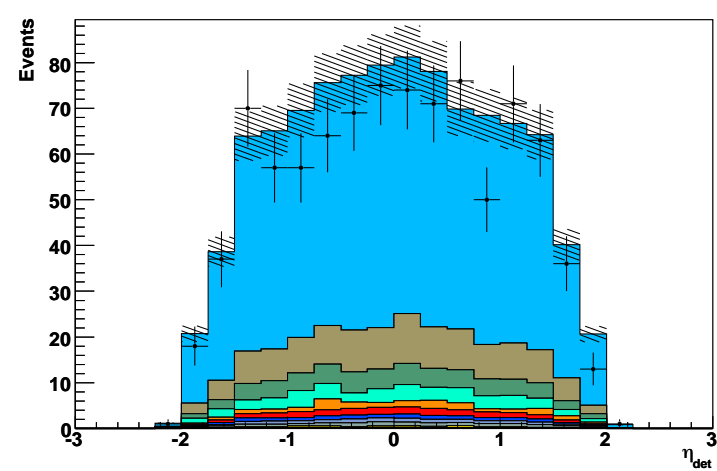

(b) 2 jet selection

Figure A.11.: Next to leading muon $\eta$ distributions.

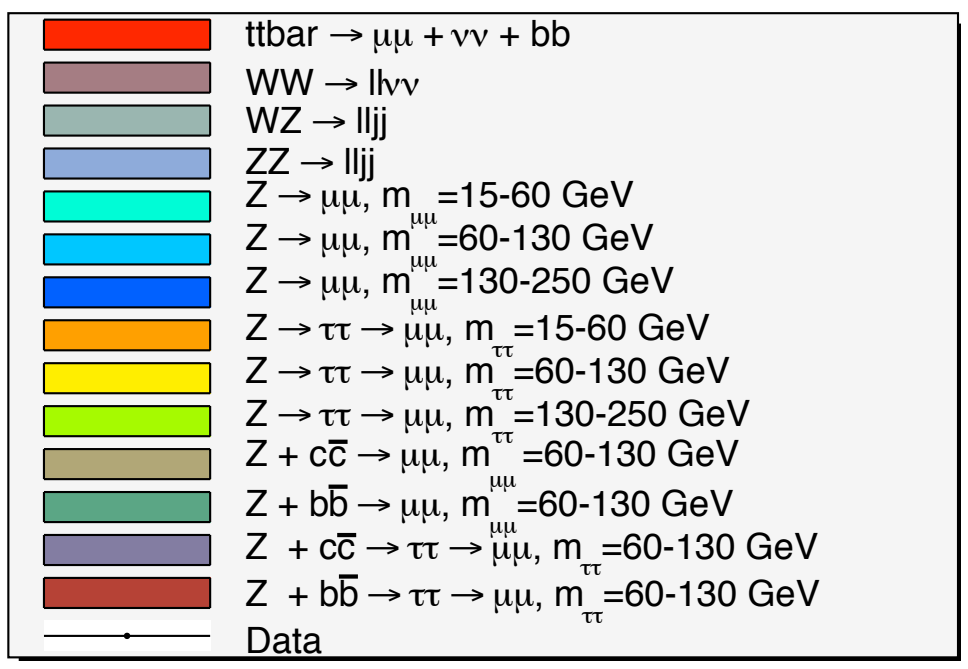

Figure A.12.: Color code used in the plots shown on this page 


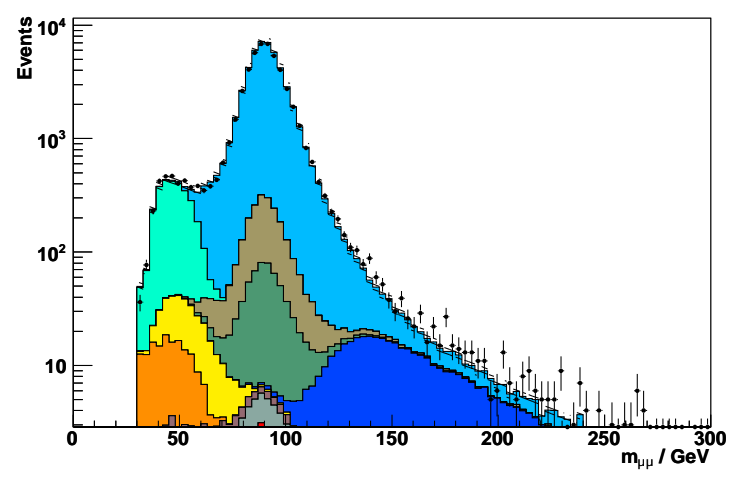

(a) 0 jet selection

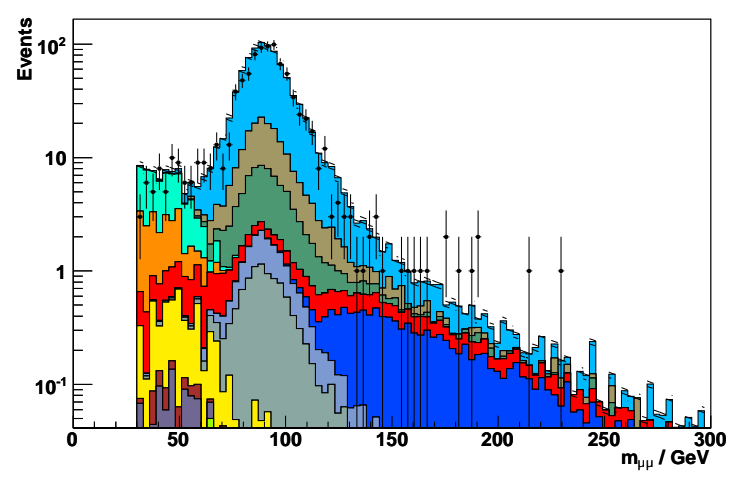

(b) 2 jet selection

Figure A.13.: Dimuon invariant mass distributions.

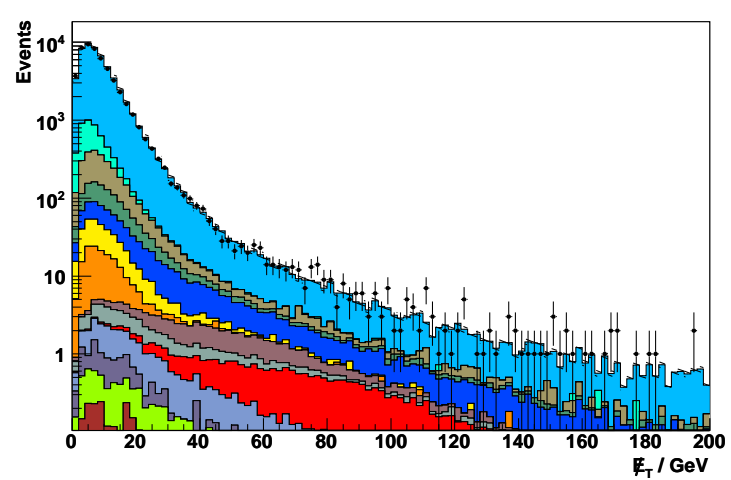

(a) 0 jet selection

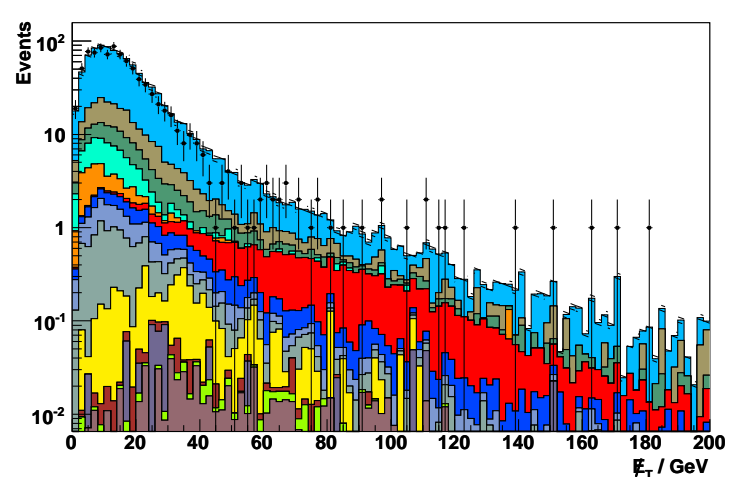

(b) 2 jet selection

Figure A.14.: $\not_{T}$ distributions.

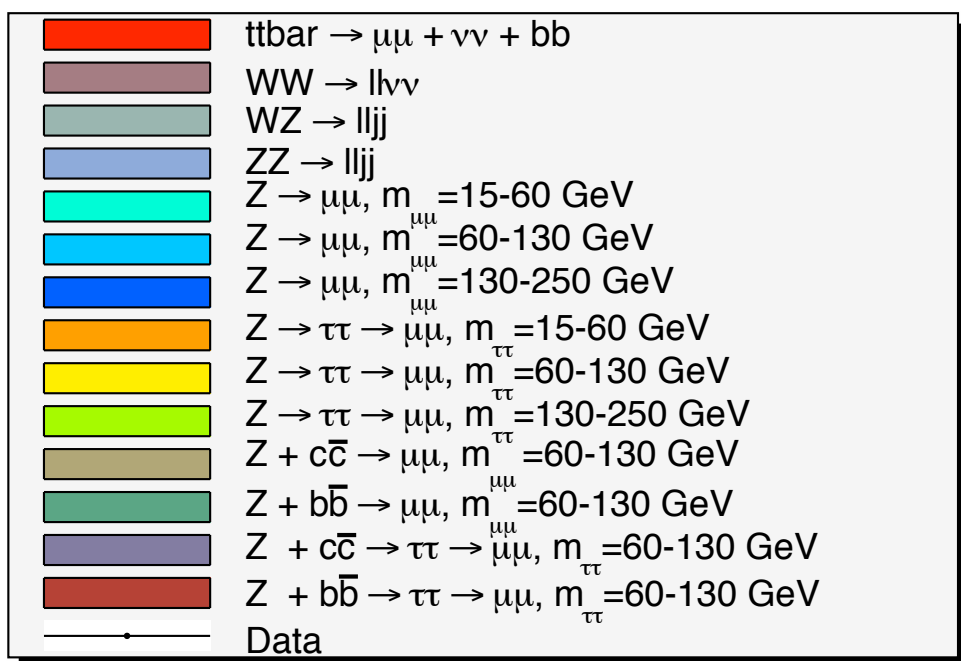

Figure A.15.: Color code used in the plots shown on this page 


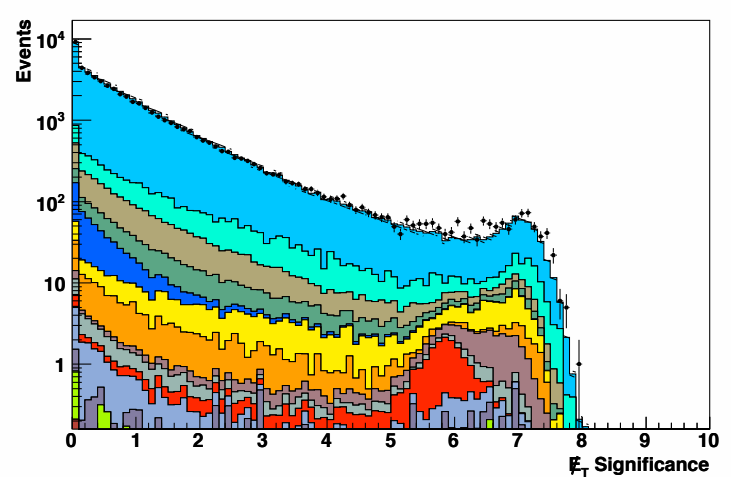

(a) 0 jet selection

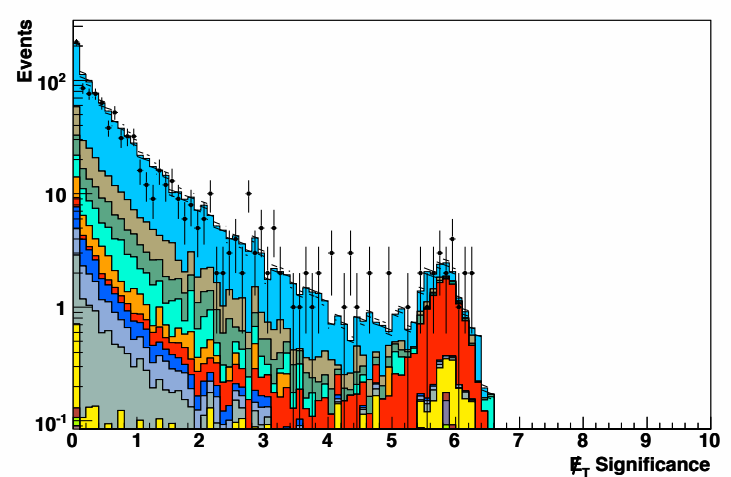

(b) 2 jet selection

Figure A.16.: $\#_{T}$ significance distributions.

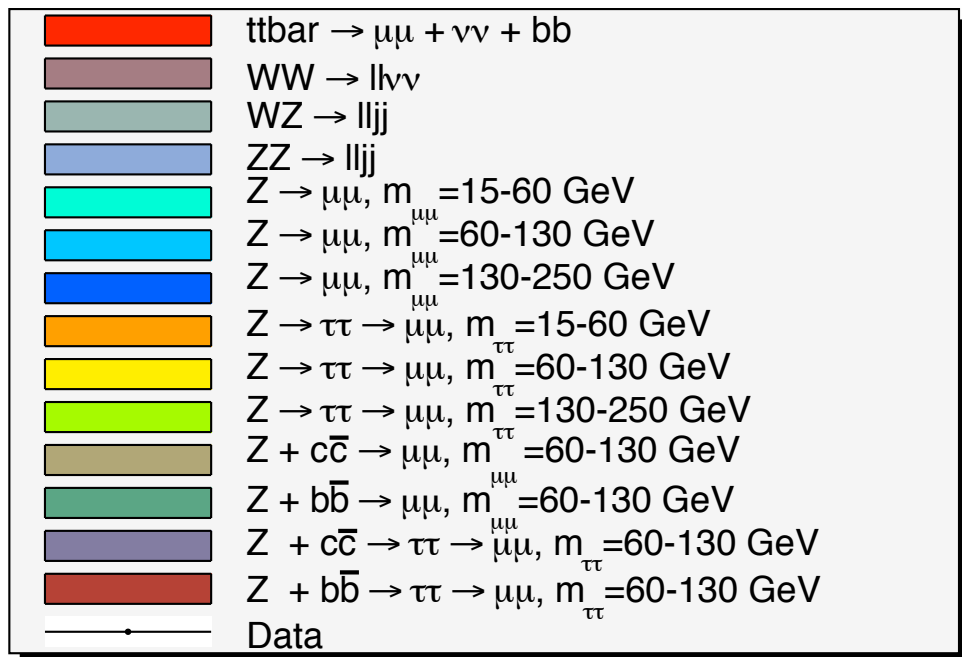

Figure A.17.: Color code used in the plots shown on this page 
APPENDIX A. DATA - MONTE CARLO COMPARISON IN THE ZERO AND TWO JET

INCLUSIVE BIN 


\section{B. $E_{\text {jet }}$ versus $E_{\text {parton }}$ Distributions and One Dimensional Profiles}




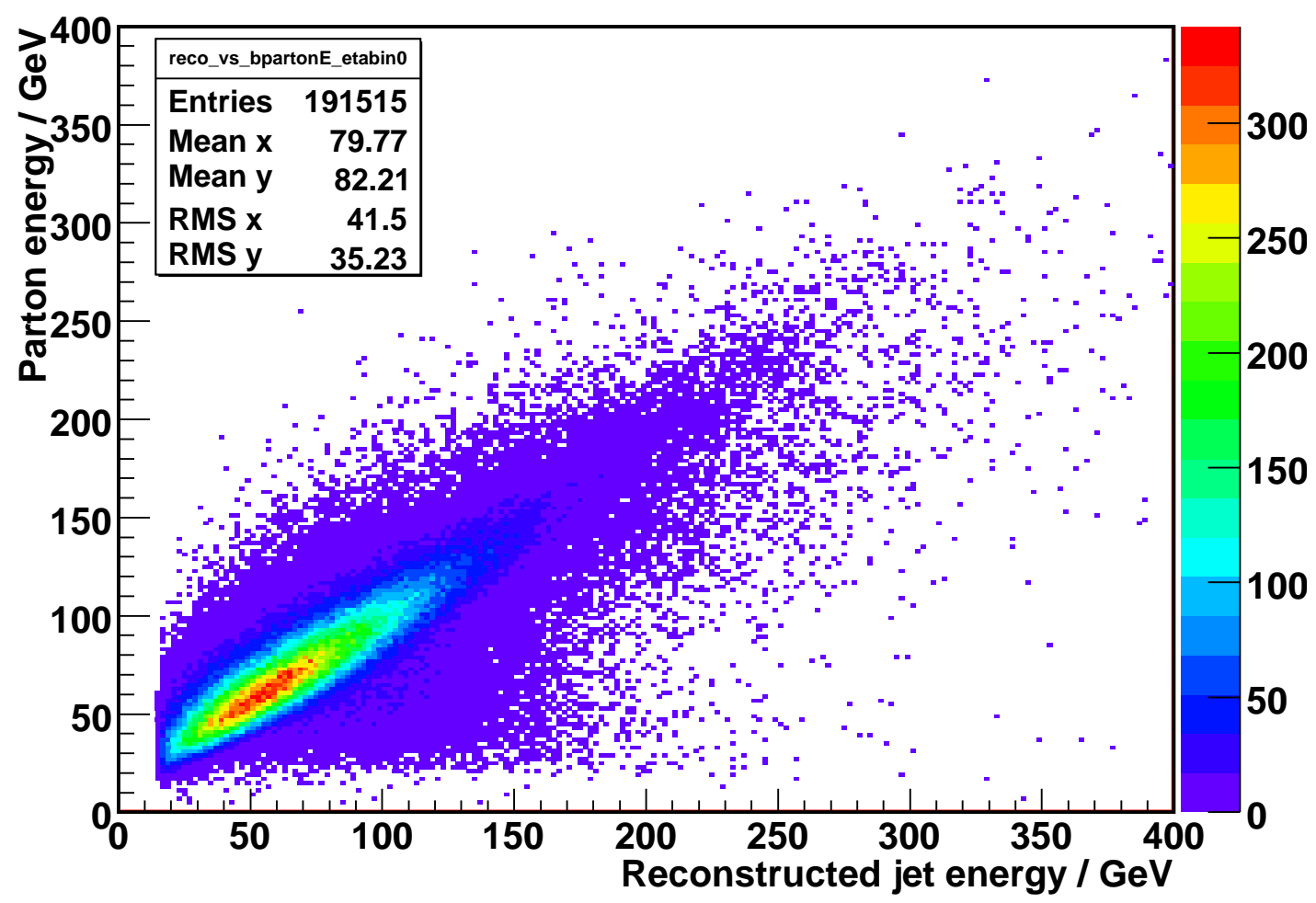

Figure B.1.: $E_{\text {jet }}$ versus $E_{\text {parton }}$ in the $0<|\eta|<0.5$ bin.

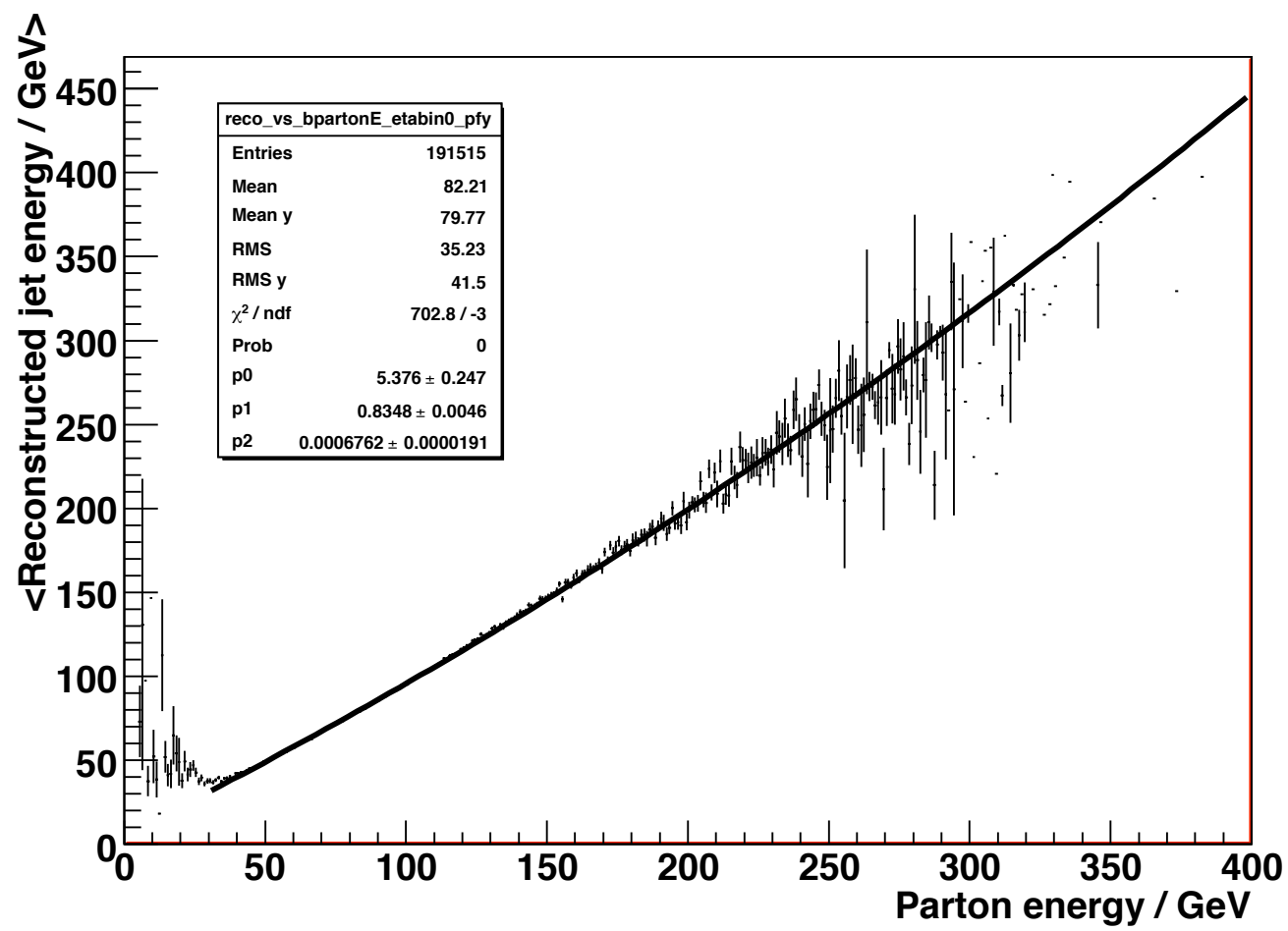

Figure B.2.: $\left\langle E_{\text {jet }}\right\rangle$ vs. $E_{\text {parton }}$ histogram derived from Fig. B.1. The solid line is the result of the fit in the shown region of $30 \mathrm{GeV}<\mathrm{E}_{\text {parton }}<400 \mathrm{GeV}$. 


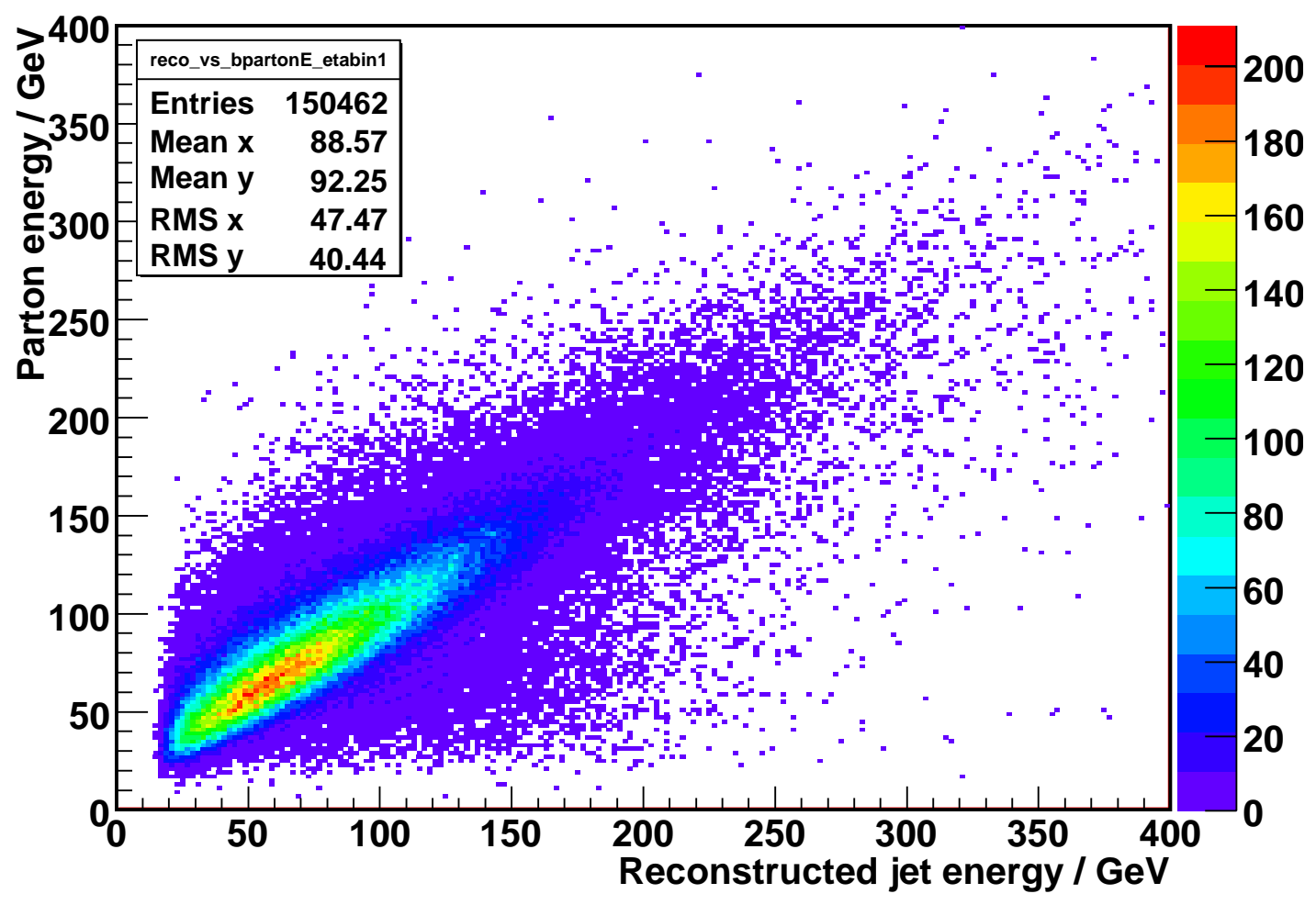

Figure B.3.: $E_{\text {jet }}$ versus $E_{\text {parton }}$ in the $0.5<|\eta|<1.0$ bin.

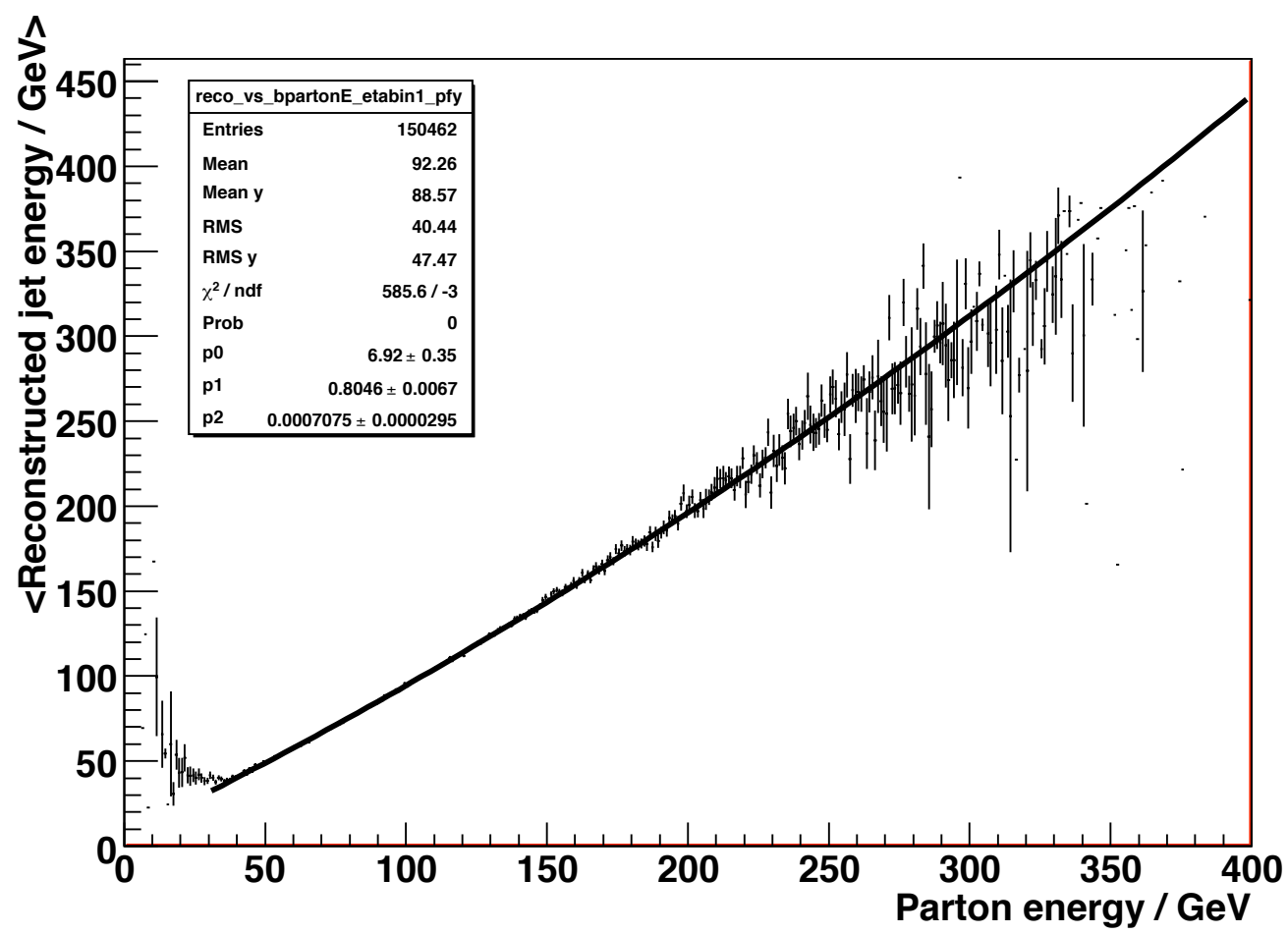

Figure B.4.: $\left\langle E_{\text {jet }}\right\rangle$ vs. $E_{\text {parton }}$ histogram derived from Fig. B.3. The solid line is the result of the fit in the shown region of $30 \mathrm{GeV}<\mathrm{E}_{\text {parton }}<400 \mathrm{GeV}$. 


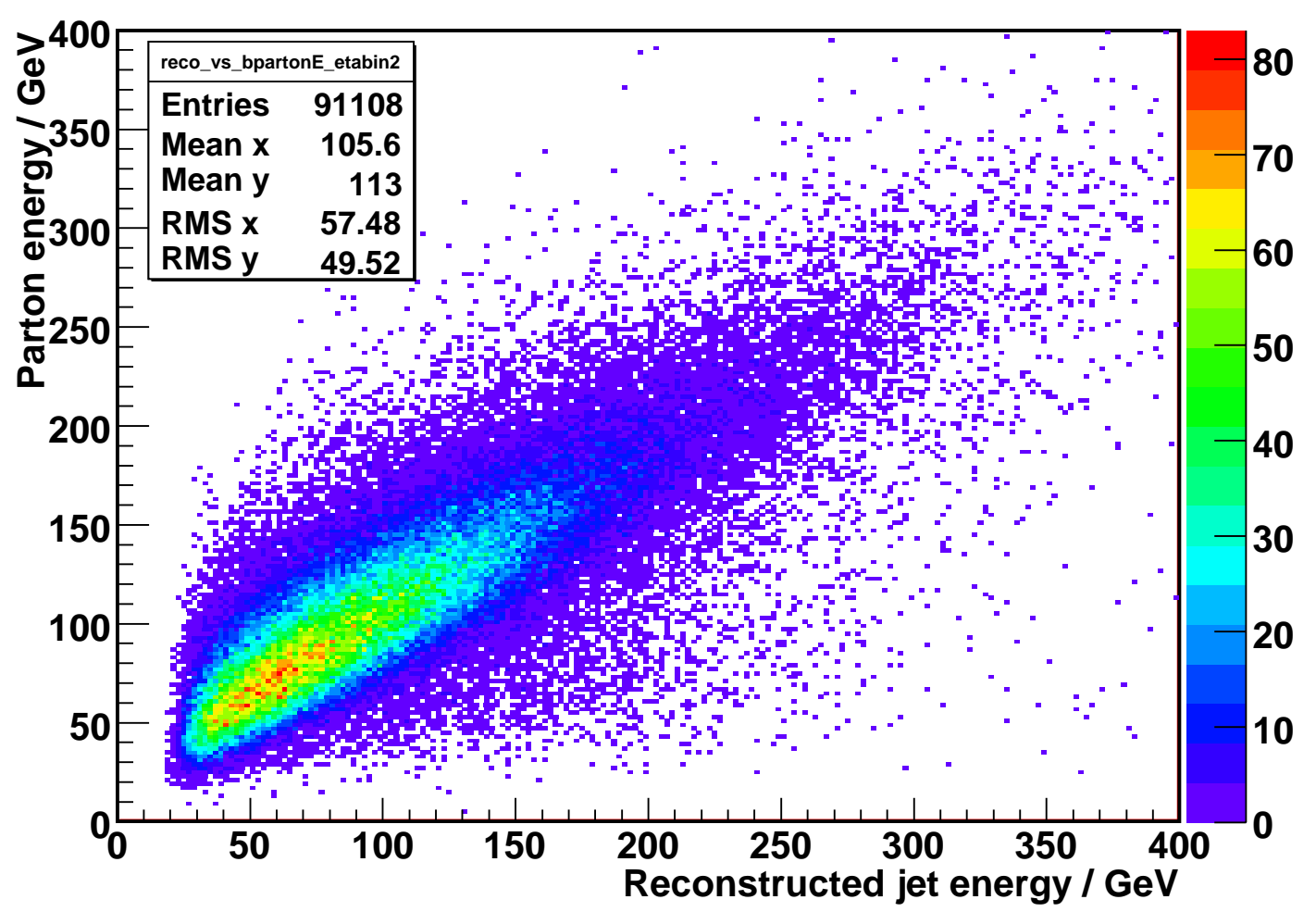

Figure B.5.: $E_{\text {jet }}$ versus $E_{\text {parton }}$ in the $1.0<|\eta|<1.5$ bin.

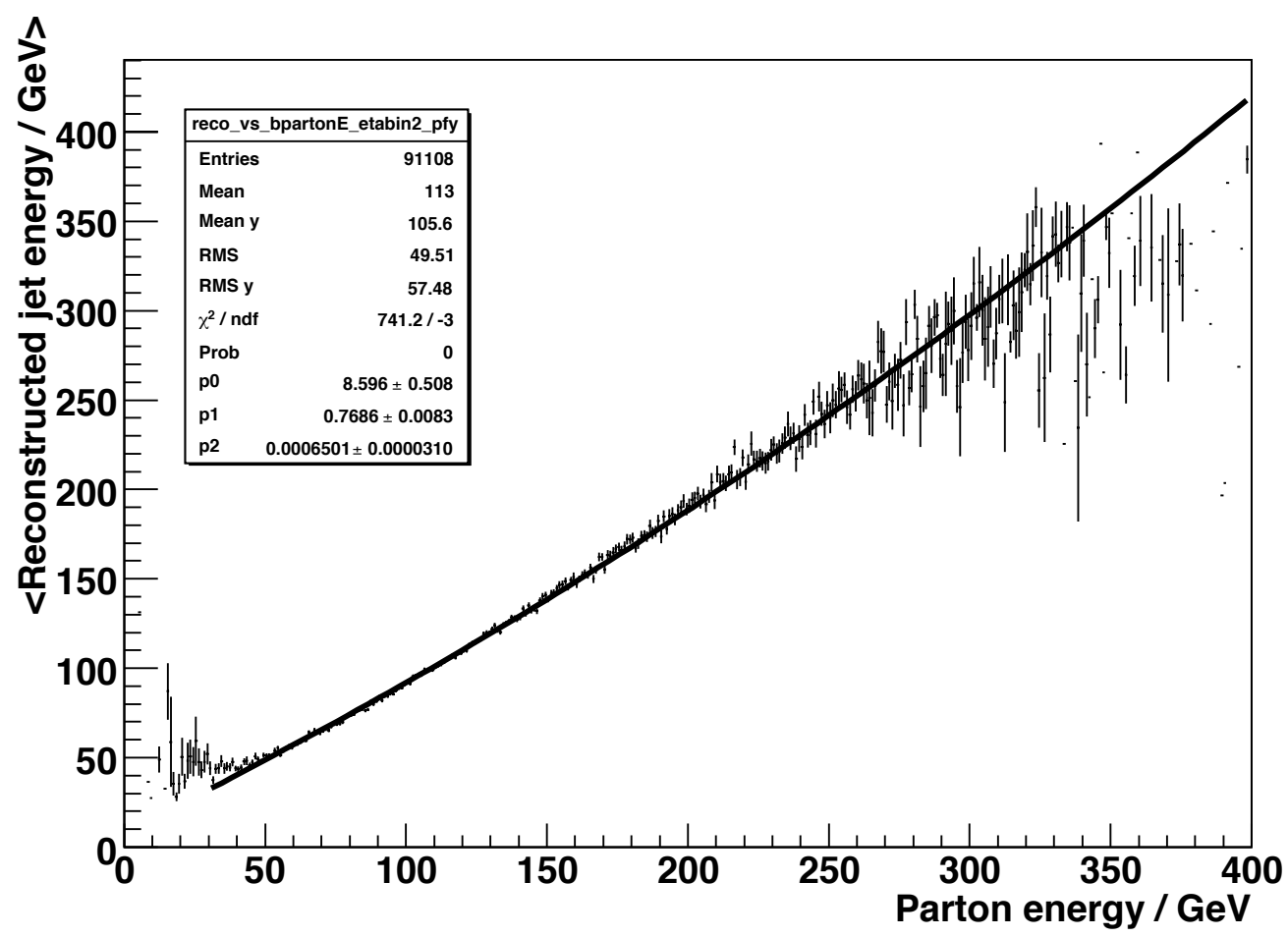

Figure B.6.: $\left\langle E_{\text {jet }}\right\rangle$ vs. $E_{\text {parton }}$ histogram derived from Fig. B.5. The solid line is the result of the fit in the shown region of $30 \mathrm{GeV}<\mathrm{E}_{\text {parton }}<400 \mathrm{GeV}$. 


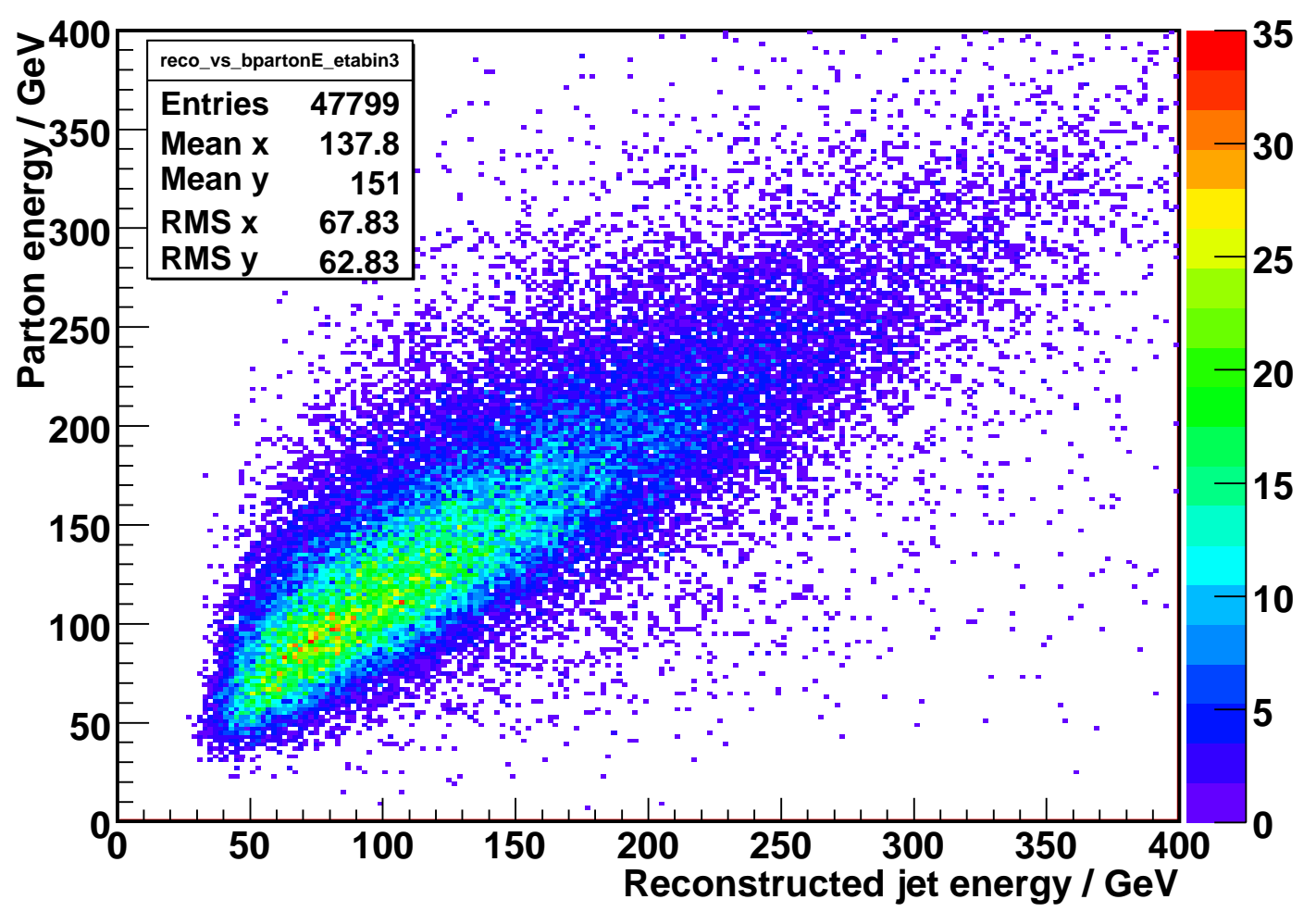

Figure B.7.: $E_{\text {jet }}$ versus $E_{\text {parton }}$ in the $1.5<|\eta|$ bin.

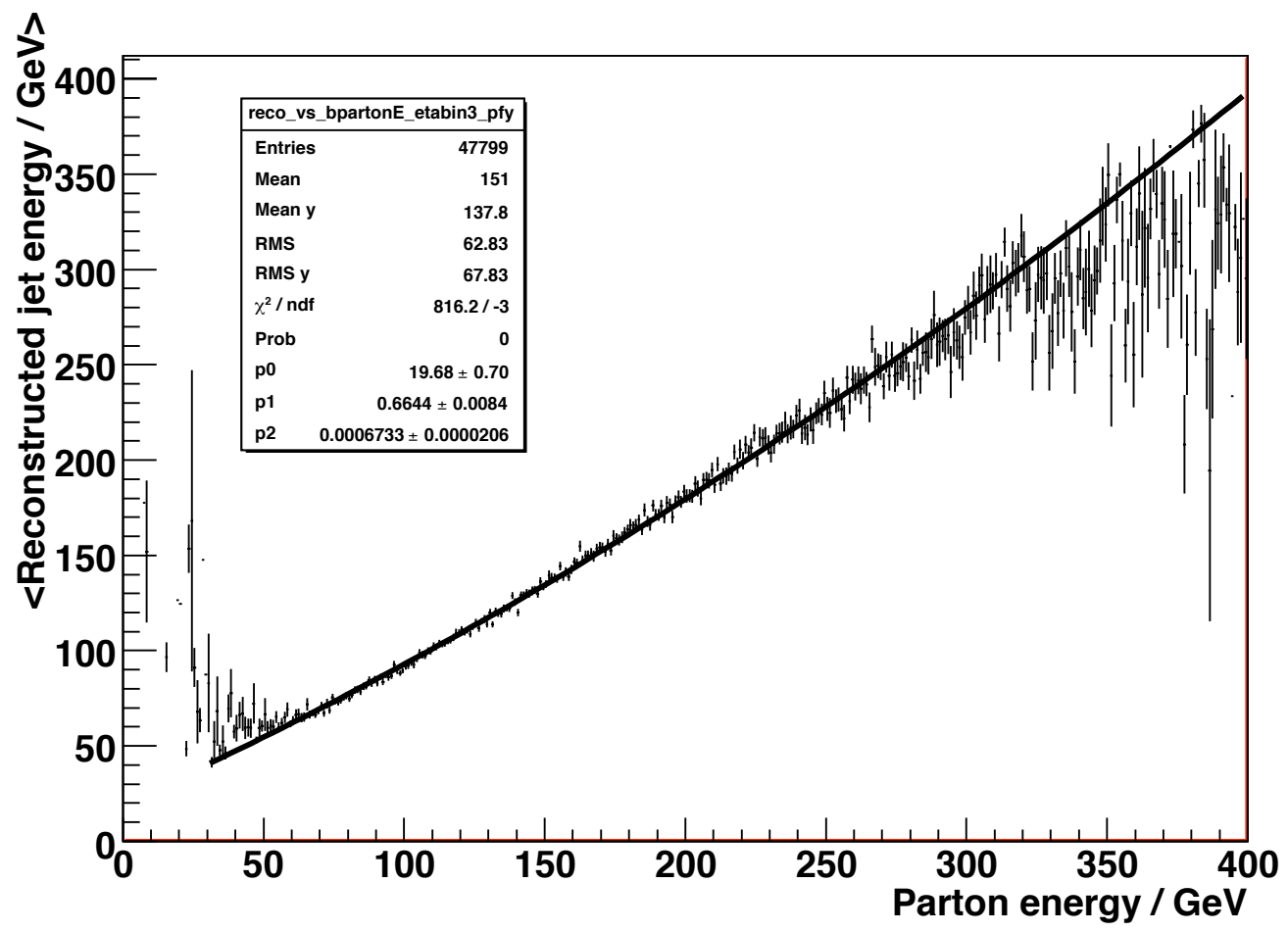

Figure B.8.: $\left\langle E_{\text {jet }}\right\rangle$ vs. $E_{\text {parton }}$ histogram derived from Fig. B.7. The solid line is the result of the fit in the shown region of $30 \mathrm{GeV}<\mathrm{E}_{\text {parton }}<400 \mathrm{GeV}$. 
APPENDIX B. $E_{J E T}$ VERSUS $E_{P A R T O N}$ DISTRIBUTIONS AND ONE DIMENSIONAL

PROFILES 
C. Calibration Plots of the Spin Correlation Measurement for Various Input Scenarios and Ensemble Tests

C.1. Calibration curves 


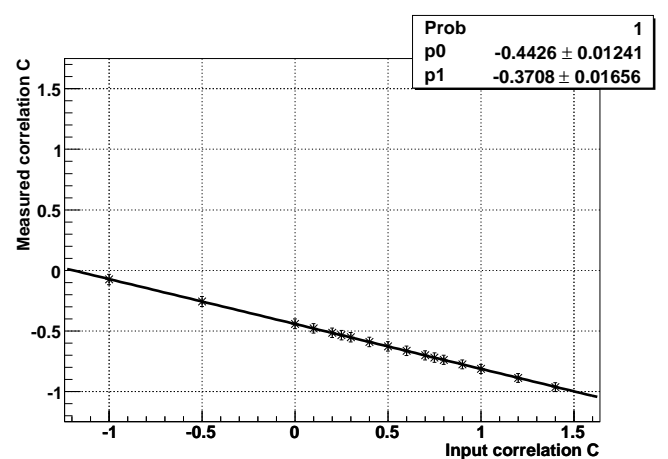

(a) t, W masses known exactly

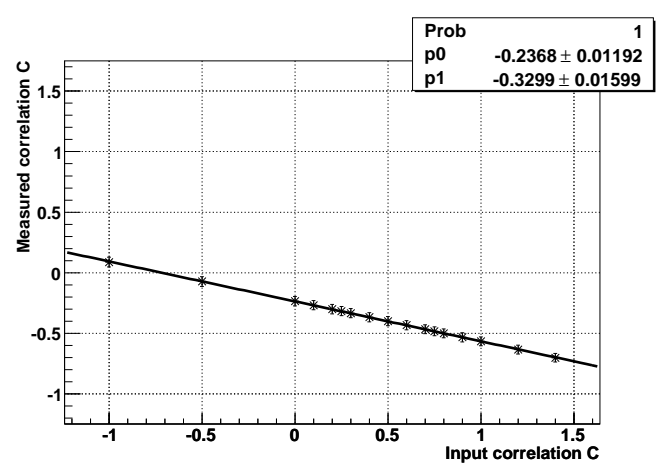

(c) t, W pole mass assumed, both $b \bar{b}$ permutations

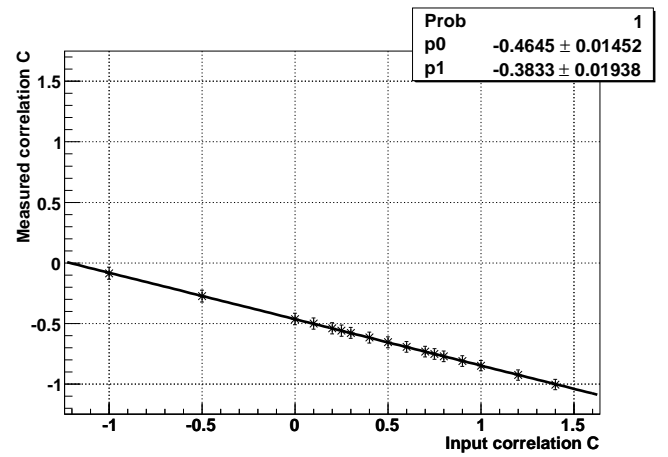

(e) Reconstructed $E_{T}$

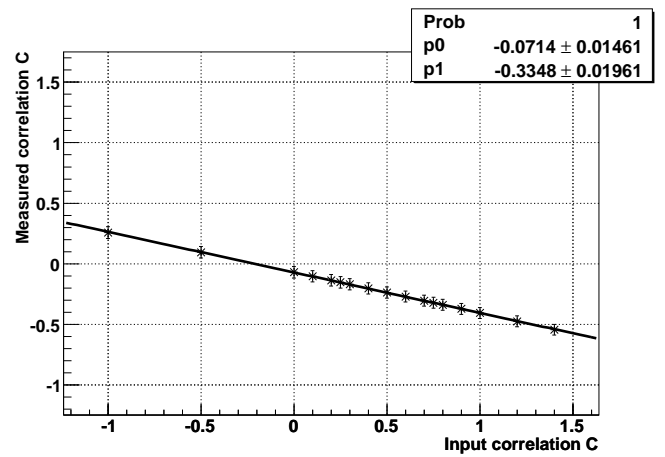

(g) Reconstructed b-jets (using leading \& ntl jet, both permutations)

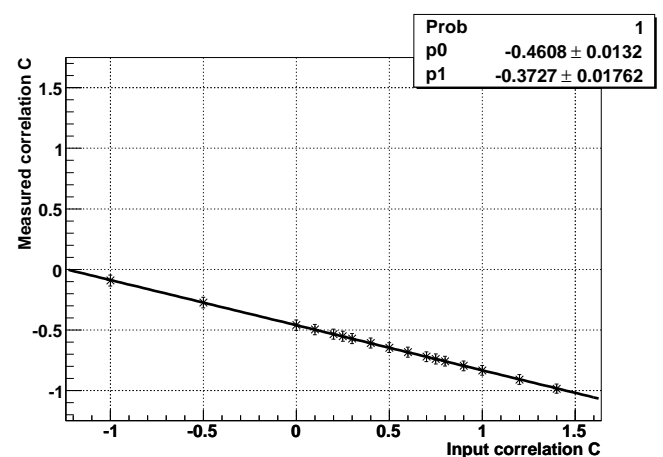

(b) t, W pole mass assumed

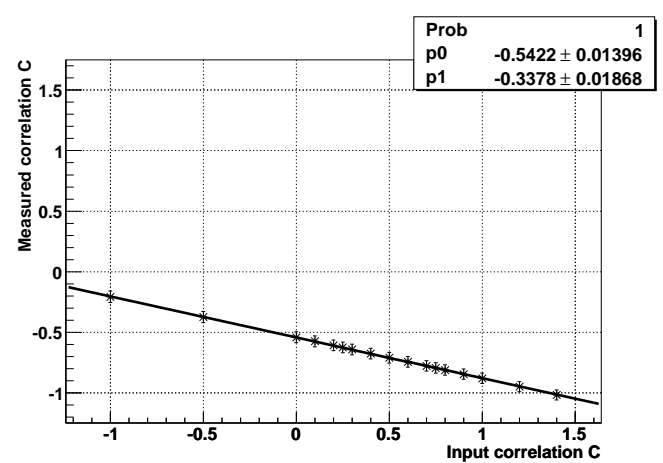

(d) Reconstructed leptons

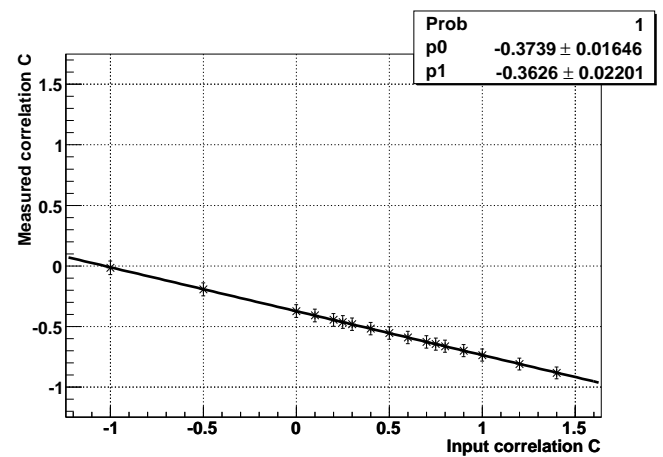

(f) Reconstructed b-jets (parton matched, PLC)

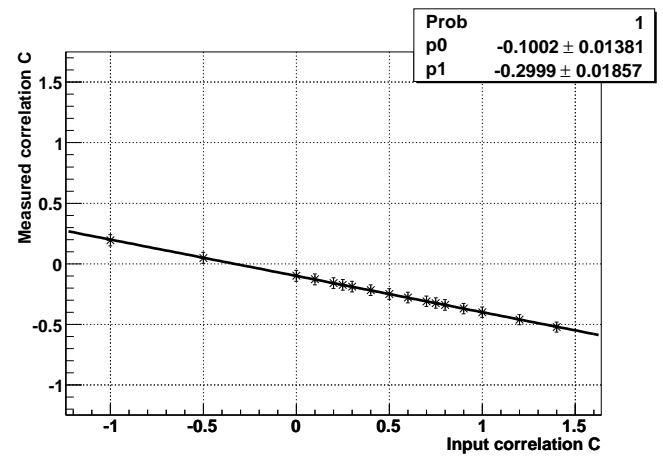

(h) Not parton - reconstructed object matching (both permutations)

Figure C.1.: Calibration plots for the measurement of $C_{\ell b}$. The letters correspond to Tab. 7.2 indicating the type of inputs to the kinematic solver. (h) uses no MC truth information and hence resembles the measurement in data. 


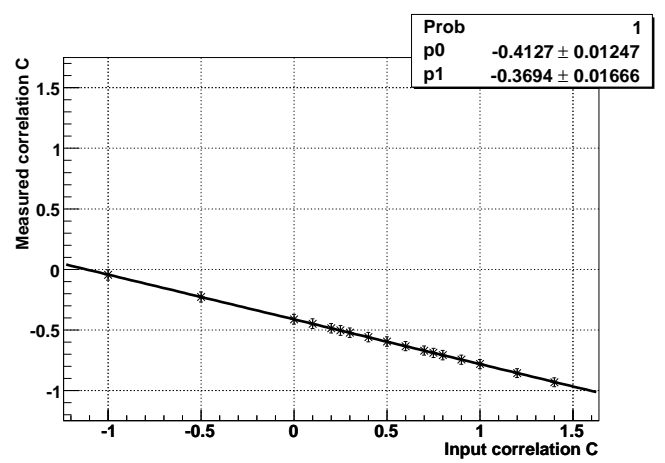

(a) t, W masses known exactly

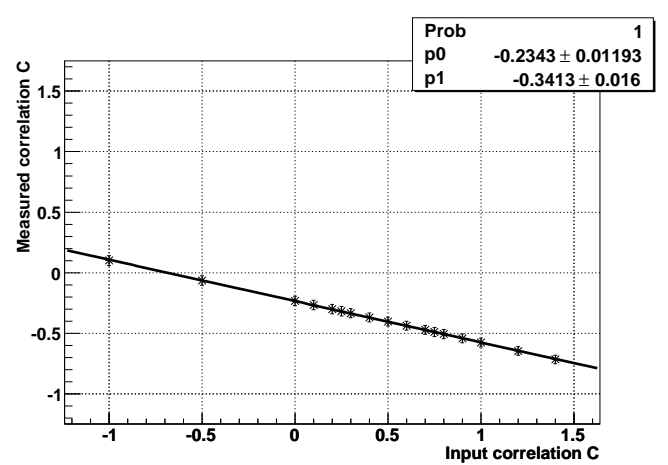

(c) t, W pole mass assumed, both $b \bar{b}$ permutations

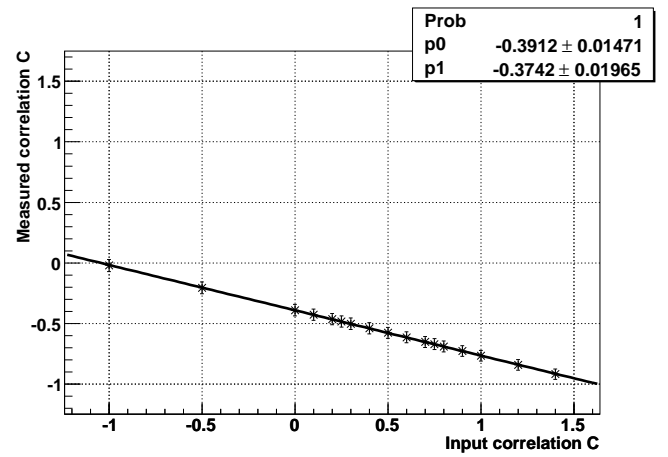

(e) Reconstructed $\not_{T}$

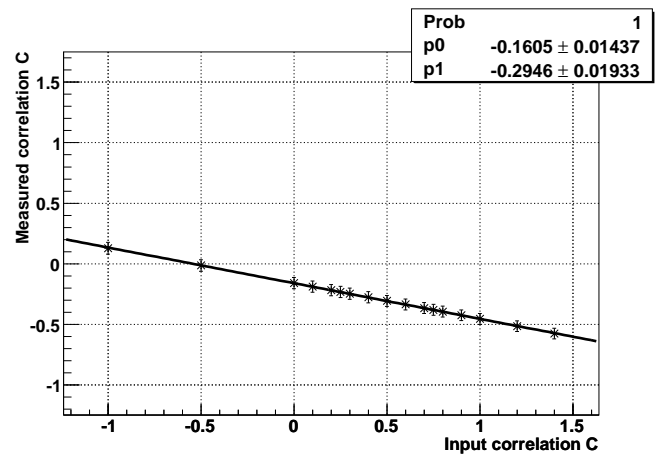

(g) Reconstructed b-jets (using leading \& ntl jet, both permutations)

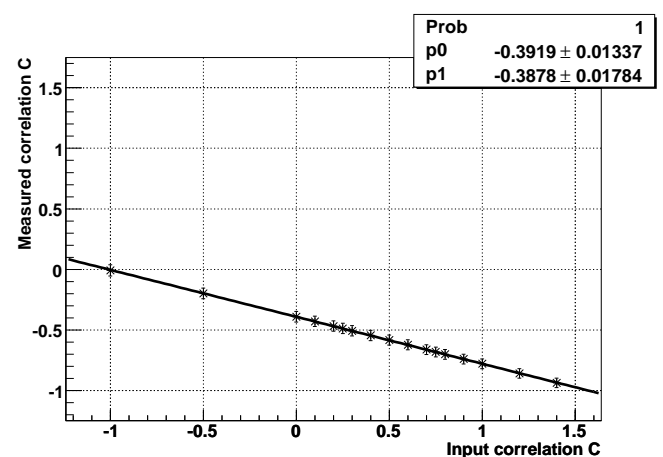

(b) t, W pole mass assumed

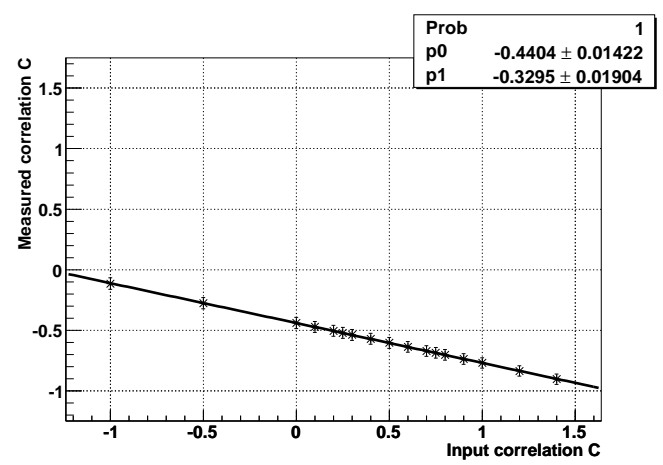

(d) Reconstructed leptons

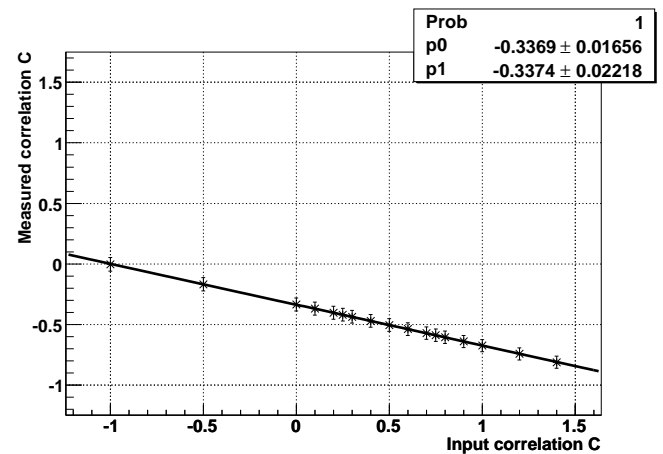

(f) Reconstructed b-jets (parton matched, PLC)

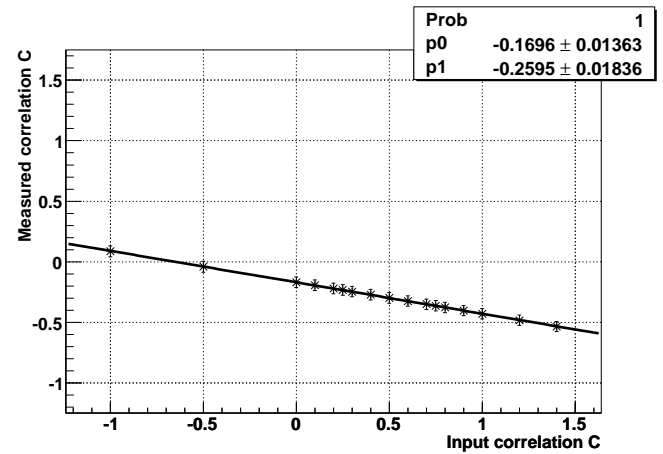

(h) Not parton - reconstructed object matching (both permutations)

Figure C.2.: Calibration plots for the measurement of $C_{\overline{\ell b}}$. The letters correspond to Tab. 7.2 indicating the type of inputs to the kinematic solver. (h) uses no MC truth information and hence resembles the measurement in data. 


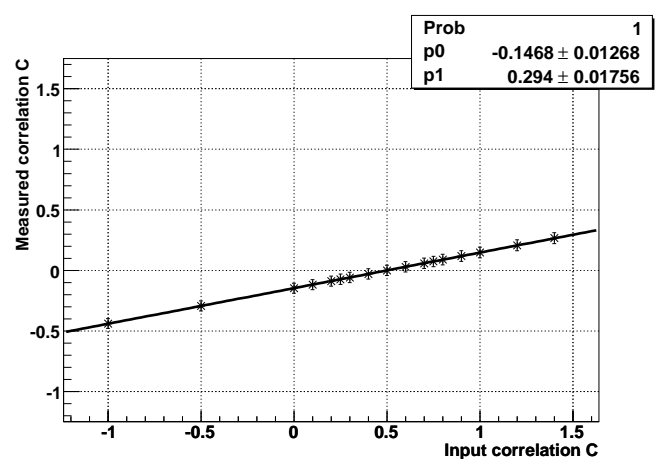

(a) t, W masses known exactly

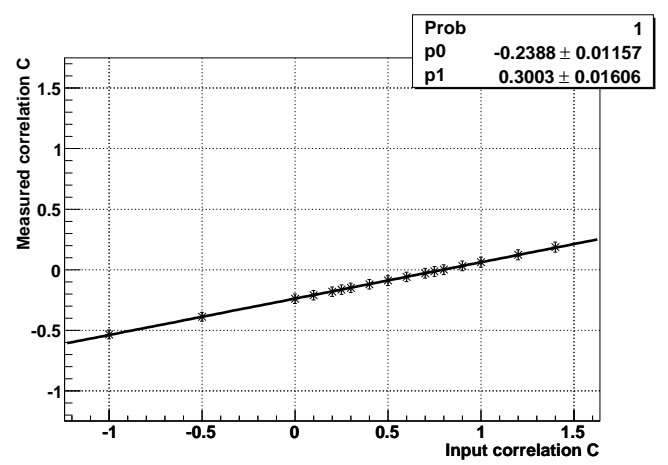

(c) t, W pole mass assumed, both $b \bar{b}$ permutations

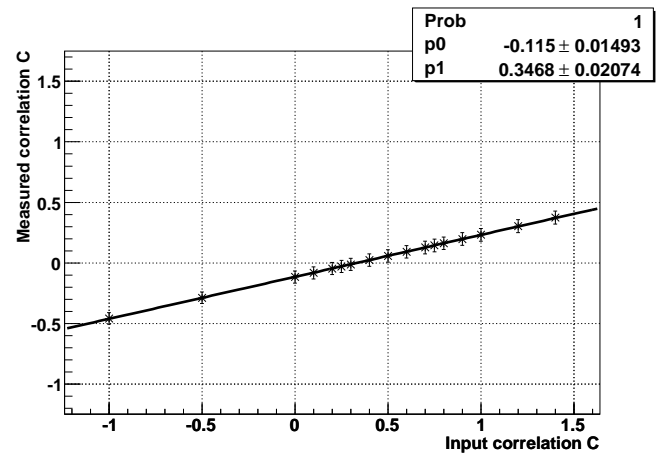

(e) Reconstructed $\not_{T}$

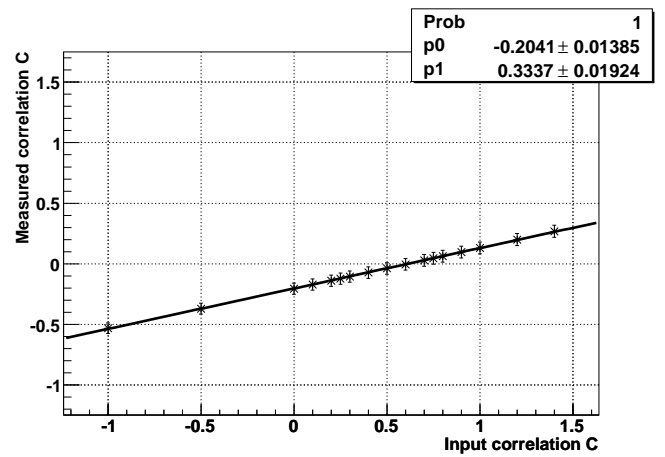

(g) Reconstructed b-jets (using leading \& ntl jet, both permutations)

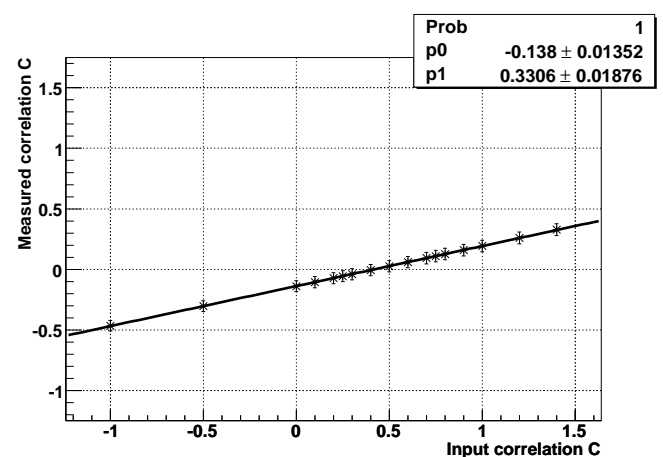

(b) t, W pole mass assumed

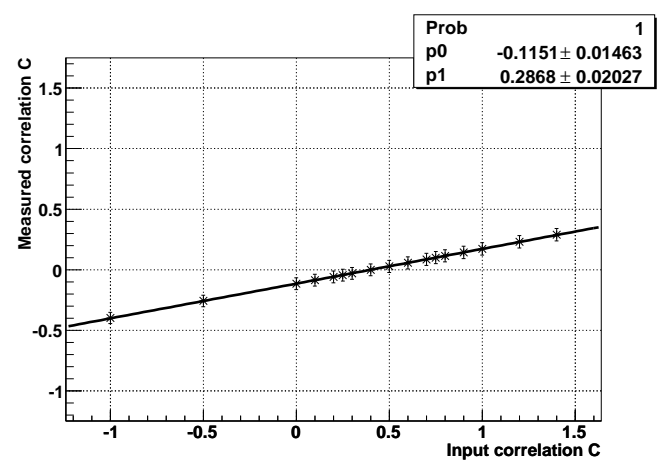

(d) Reconstructed leptons

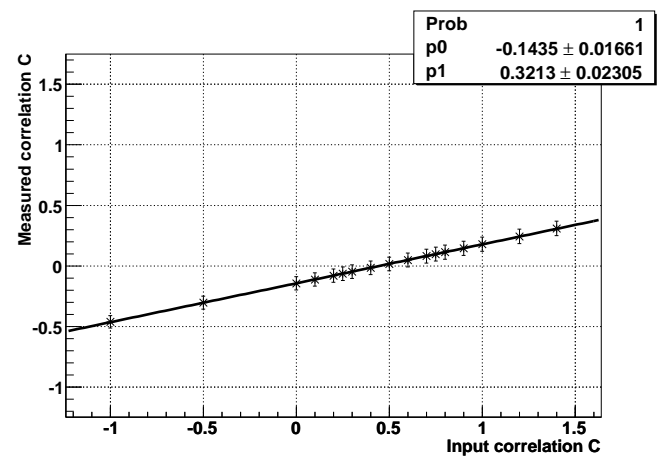

(f) Reconstructed b-jets (parton matched, PLC)

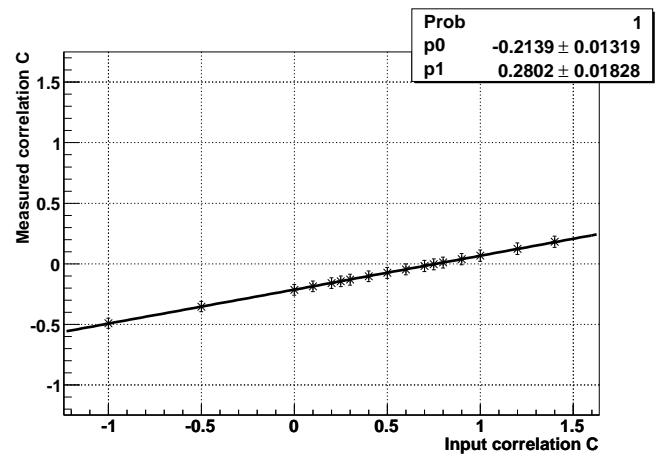

(h) Not parton - reconstructed object matching (both permutations)

Figure C.3.: Calibration plots for the measurement of $C_{W^{+} W^{-}}$. The letters correspond to Tab. 7.2 indicating the type of inputs to the kinematic solver. (h) uses no $\mathrm{MC}$ truth information and hence resembles the measurement in data. 


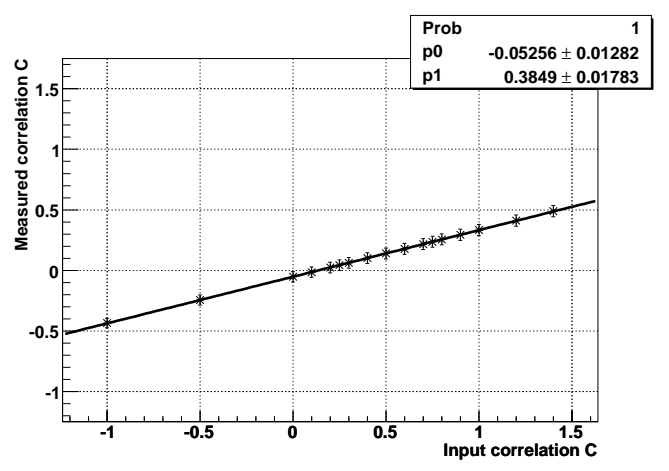

(a) t, W masses known exactly

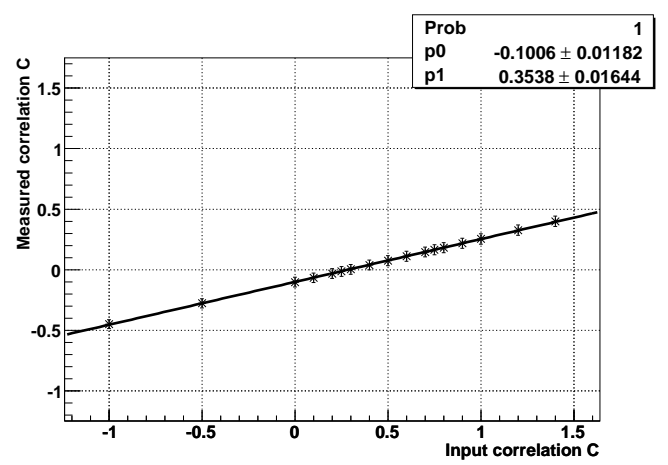

(c) t, W pole mass assumed, both $b \bar{b}$ permutations

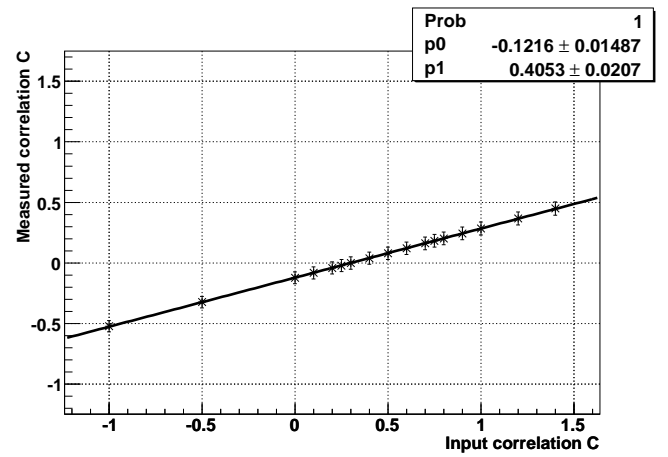

(e) Reconstructed $\#_{T}$

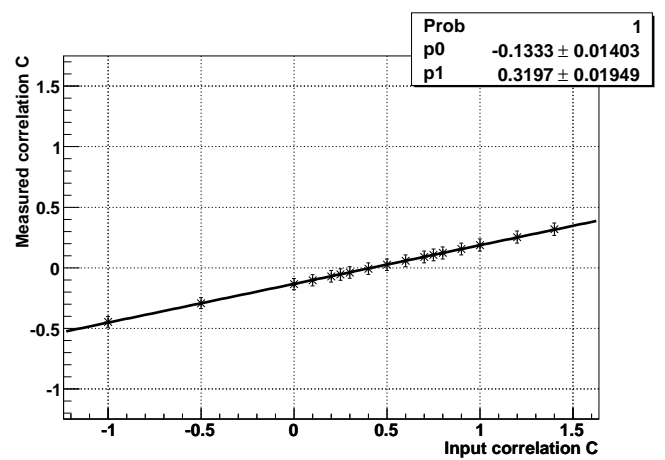

(g) Reconstructed b-jets (using leading \& ntl jet, both permutations)

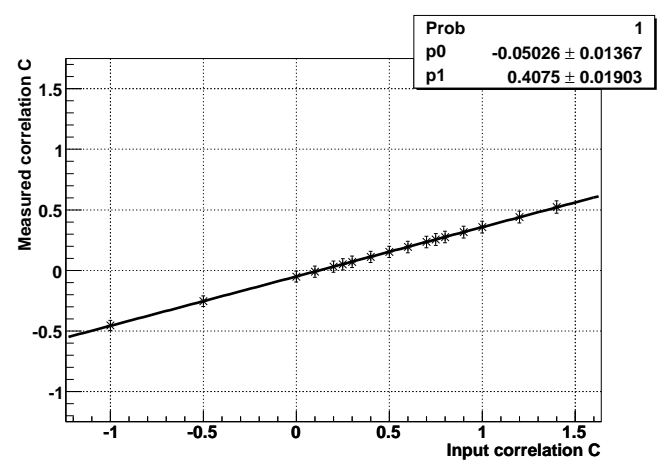

(b) t, W pole mass assumed

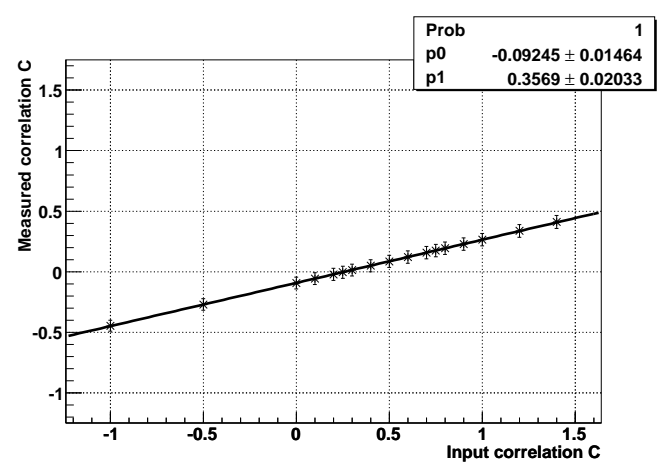

(d) Reconstructed leptons

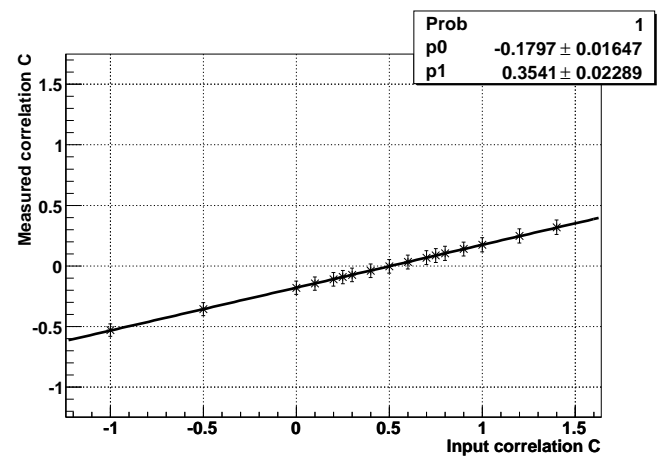

(f) Reconstructed b-jets (parton matched, PLC)

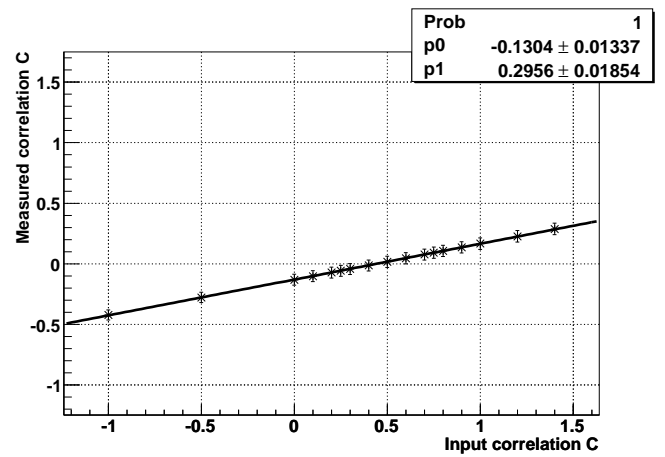

(h) Not parton - reconstructed object matching (both permutations)

Figure C.4.: Calibration plots for the measurement of $C_{W^{-} \bar{\ell}}$. The letters correspond to Tab. 7.2 indicating the type of inputs to the kinematic solver. (h) uses no $\mathrm{MC}$ truth information and hence resembles the measurement in data. 


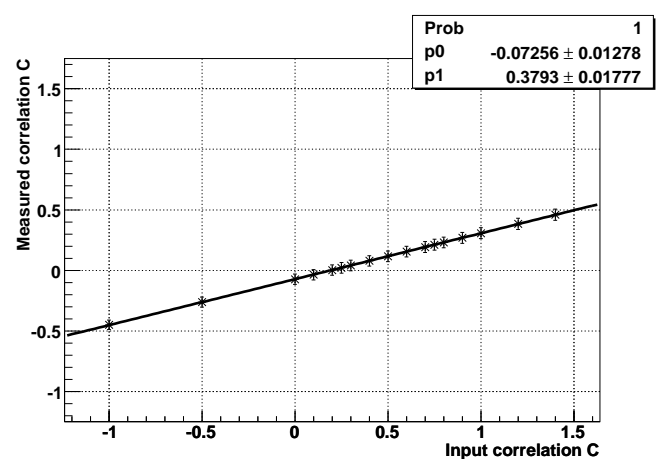

(a) t, W masses known exactly

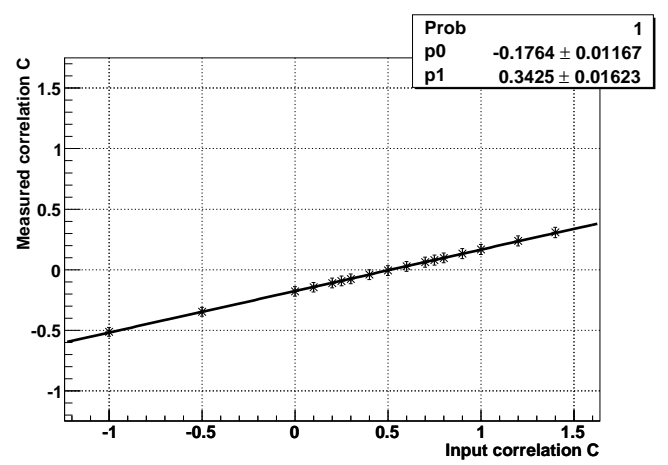

(c) t, W pole mass assumed, both $b \bar{b}$ permutations

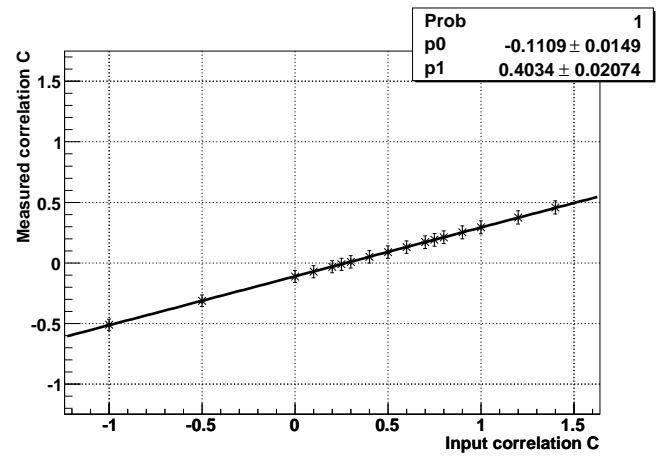

(e) Reconstructed $\not_{T}$

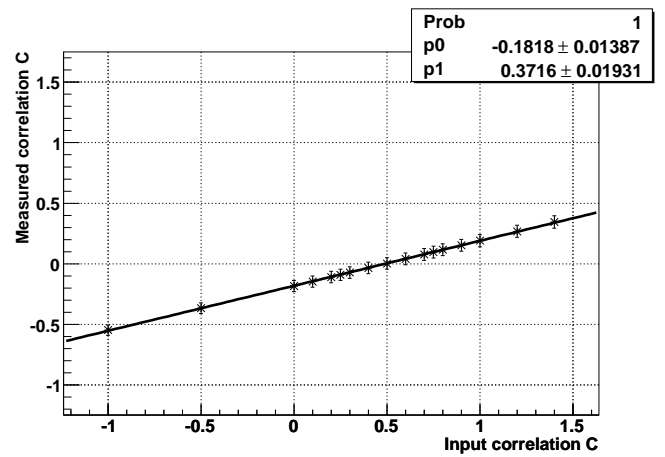

(g) Reconstructed b-jets (using leading \& ntl jet, both permutations)

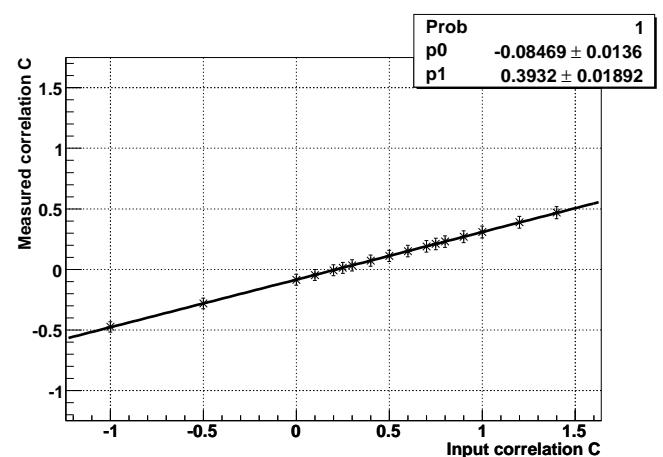

(b) t, W pole mass assumed

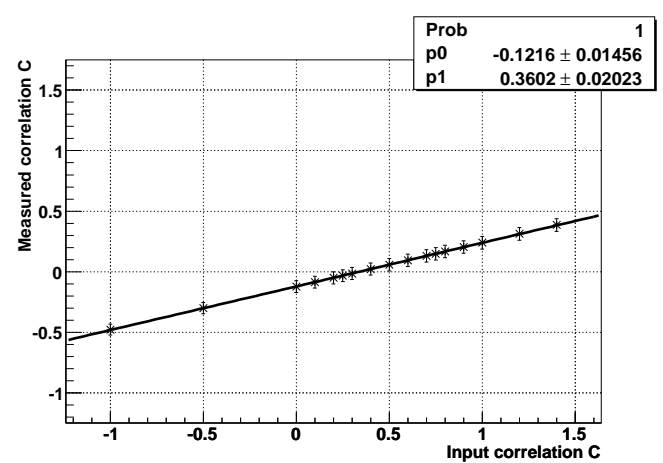

(d) Reconstructed leptons

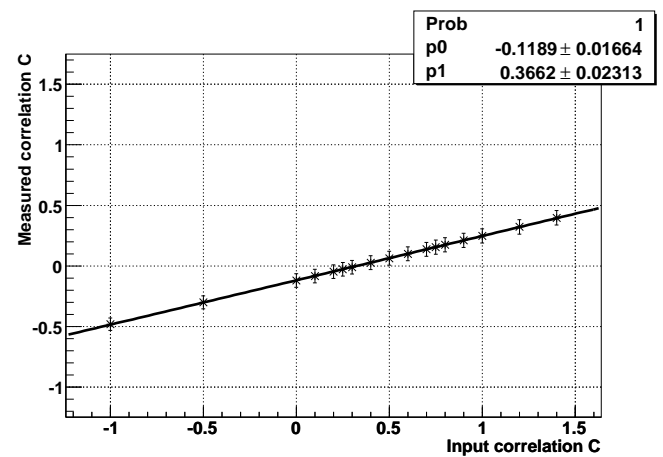

(f) Reconstructed b-jets (parton matched, PLC)

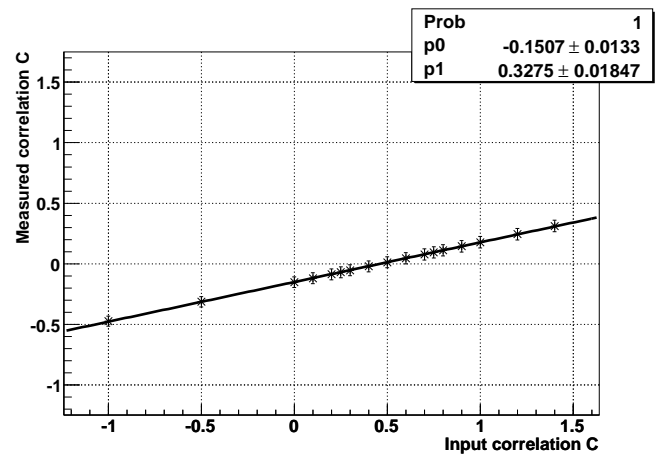

(h) Not parton - reconstructed object matching (both permutations)

Figure C.5.: Calibration plots for the measurement of $C_{W^{+} \ell}$. The letters correspond to Tab. 7.2 indicating the type of inputs to the kinematic solver. (h) uses no $\mathrm{MC}$ truth information and hence resembles the measurement in data. 


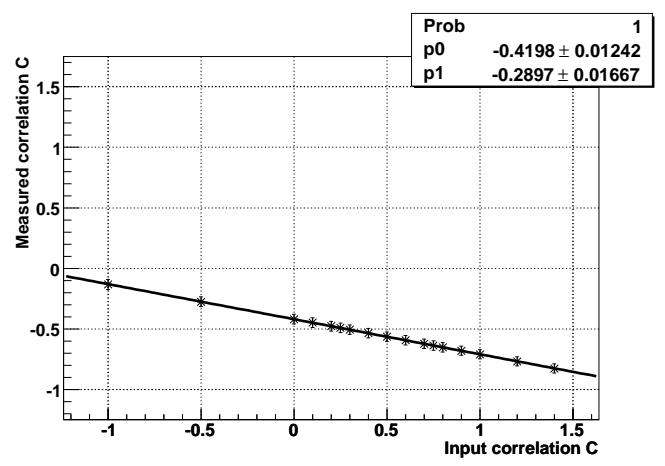

(a) t, W masses known exactly

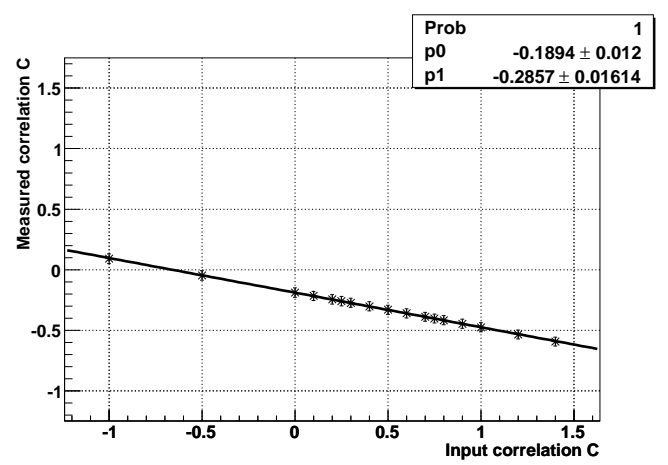

(c) t, W pole mass assumed, both $b \bar{b}$ permutations

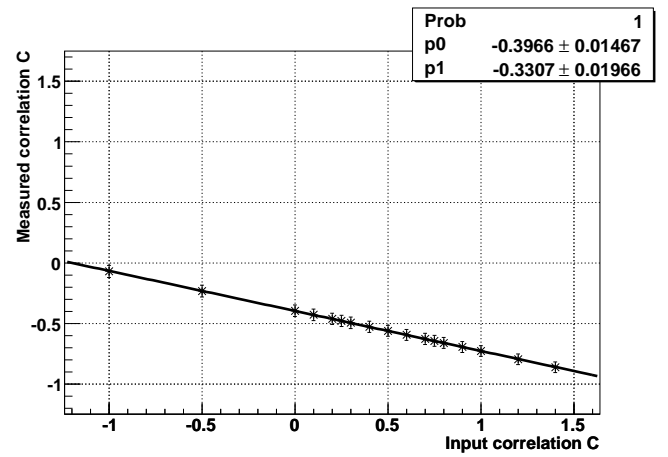

(e) Reconstructed $\not_{T}$

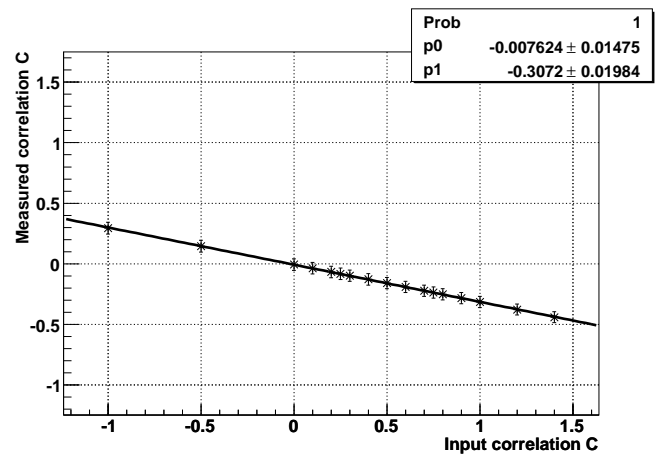

(g) Reconstructed b-jets (using leading \& ntl jet, both permutations)

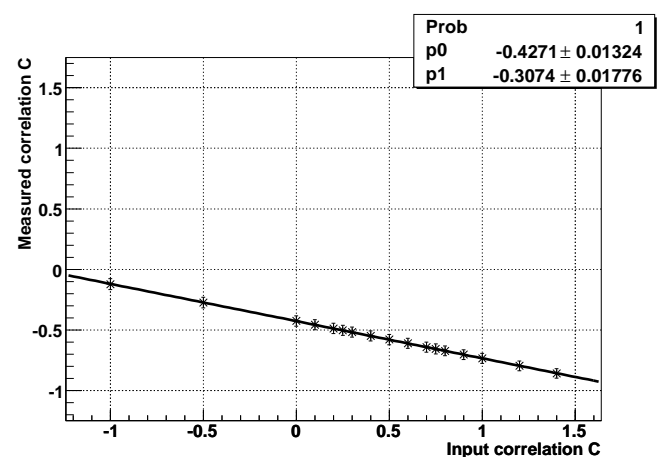

(b) t, W pole mass assumed

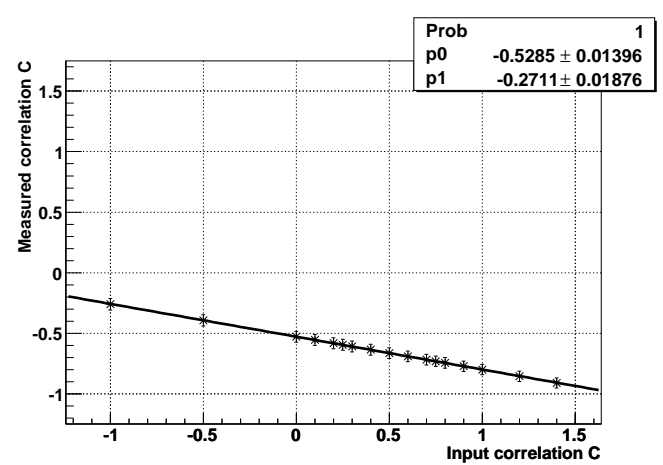

(d) Reconstructed leptons

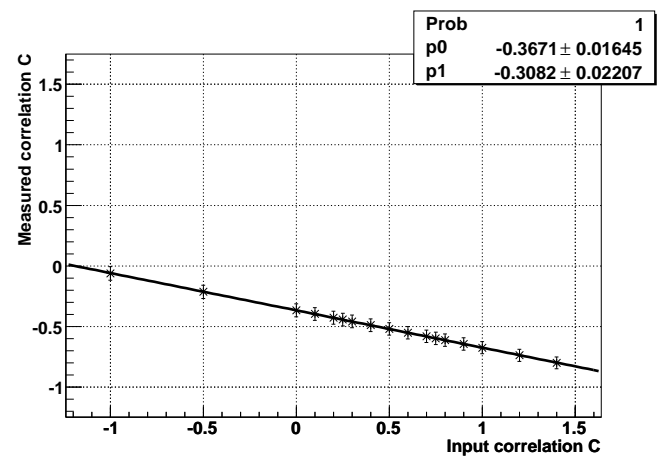

(f) Reconstructed b-jets (parton matched, PLC)

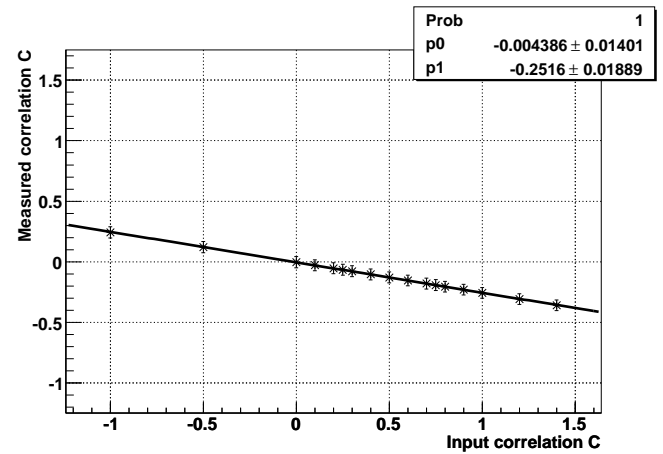

(h) Not parton - reconstructed object matching (both permutations)

Figure C.6.: Calibration plots for the measurement of $C_{W^{-} b}$. The letters correspond to Tab. 7.2 indicating the type of inputs to the kinematic solver. (h) uses no $\mathrm{MC}$ truth information and hence resembles the measurement in data. 


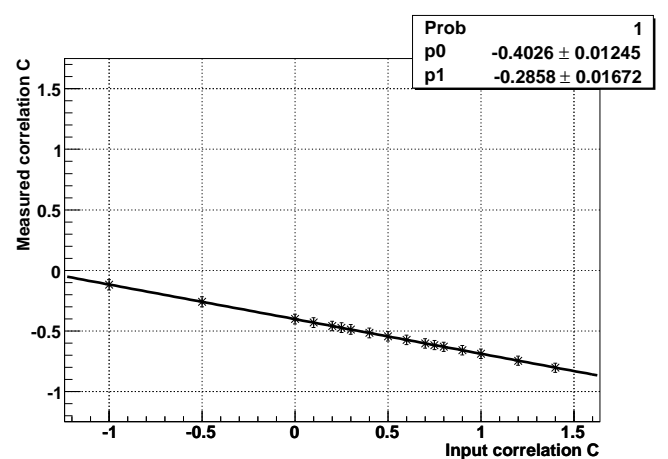

(a) t, W masses known exactly

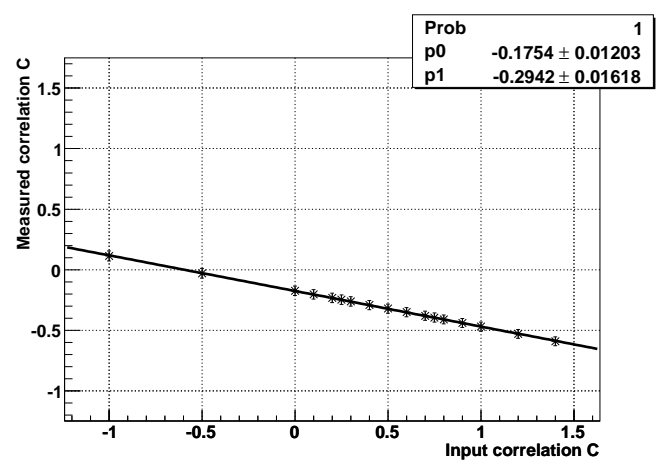

(c) t, W pole mass assumed, both $b \bar{b}$ permutations

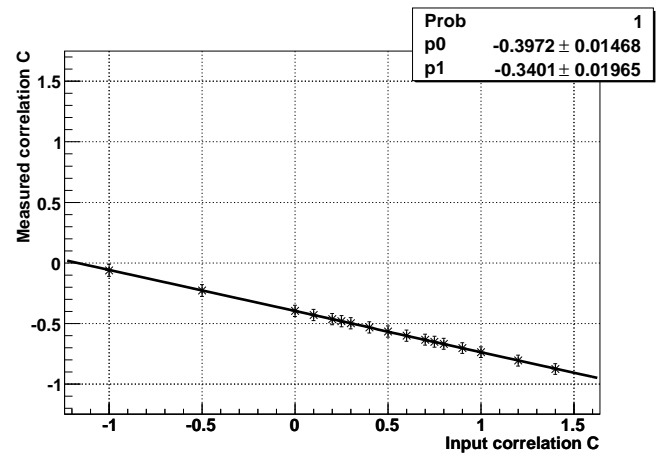

(e) Reconstructed $E_{T}$

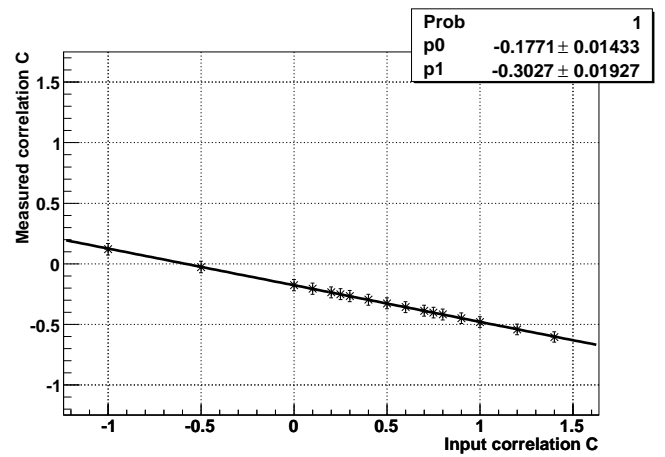

(g) Reconstructed b-jets (using leading \& ntl jet, both permutations)

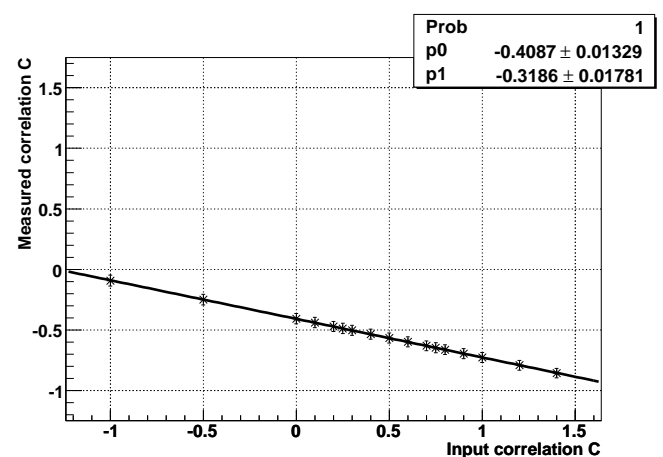

(b) t, W pole mass assumed

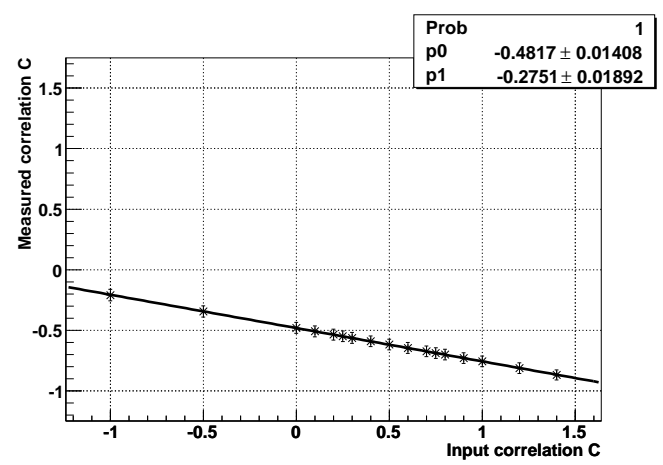

(d) Reconstructed leptons

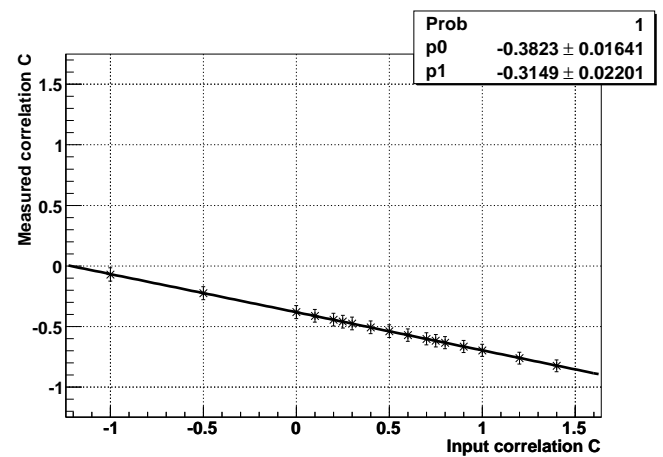

(f) Reconstructed b-jets (parton matched, PLC)

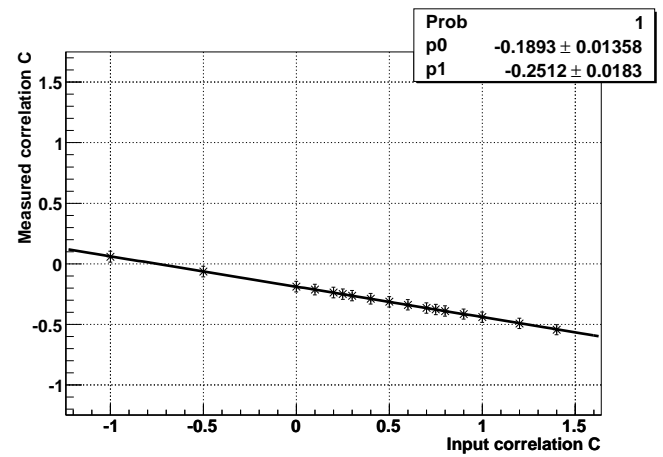

(h) Not parton - reconstructed object matching (both permutations)

Figure C.7.: Calibration plots for the measurement of $C_{W+\bar{b}}$. The letters correspond to Tab. 7.2 indicating the type of inputs to the kinematic solver. (h) uses no $\mathrm{MC}$ truth information and hence resembles the measurement in data. 


\section{C.2. Ensemble Tests}

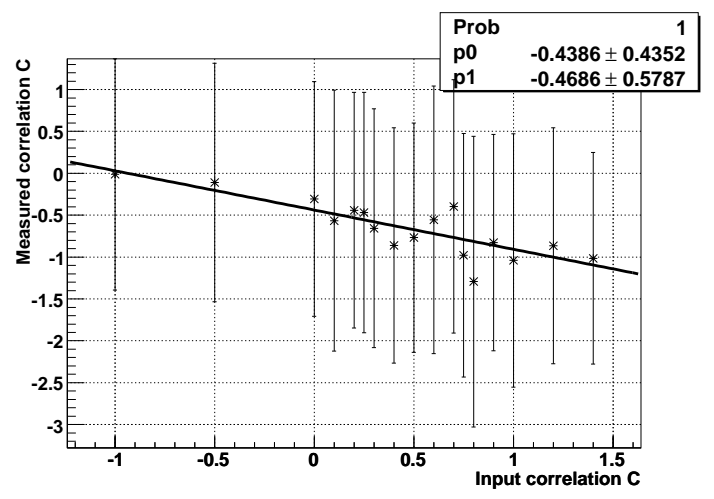

(a) t, W masses known exactly

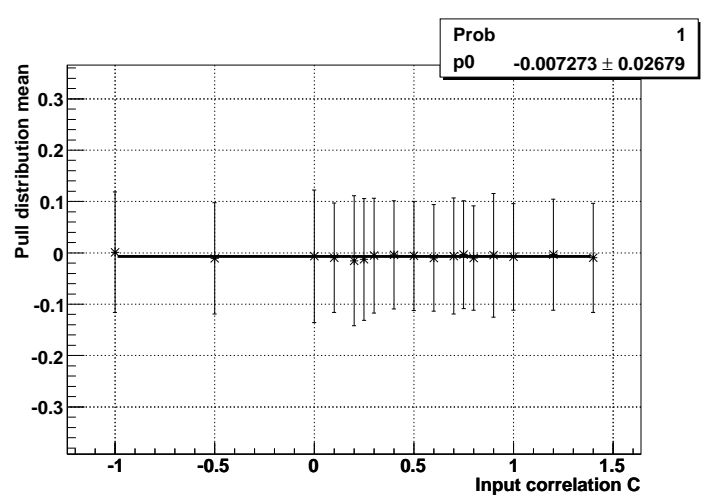

(c) Mean of the pull distribution for (b)

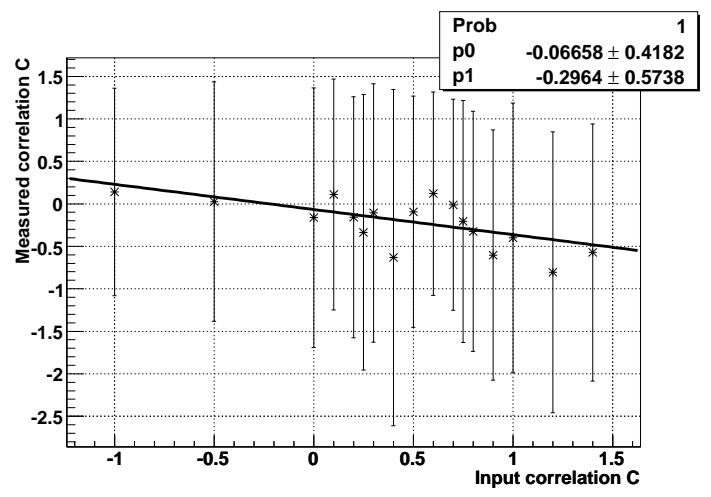

(b) Not parton - reconstructed object matching (both permutations)

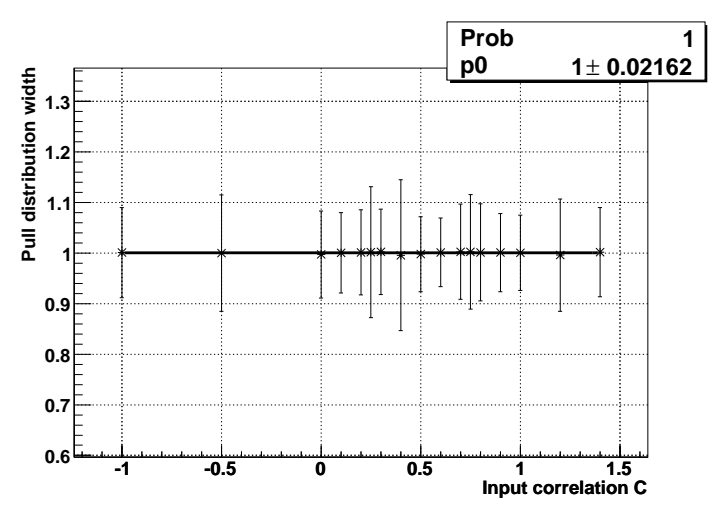

(d) Width of the pull distribution for (b)

Figure C.8.: Ensemble tests for the measurement of $C_{\ell b}$. 100 ensembles with 8 events each were generated. The error bars in the calibration curve plots indicate $\sigma_{C_{\text {meas }}^{\text {all ensembles }}}$ 


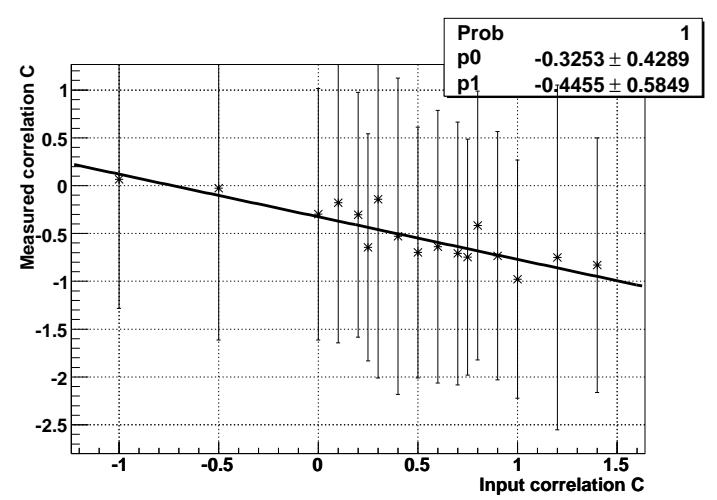

(a) t, W masses known exactly

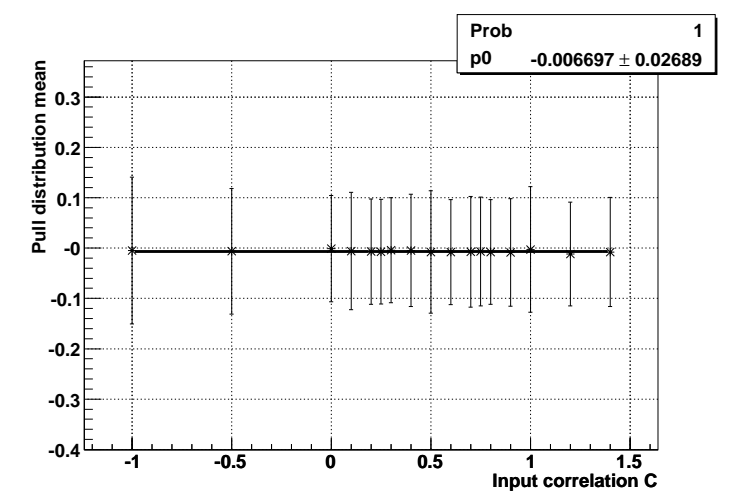

(c) Mean of the pull distribution for (b)

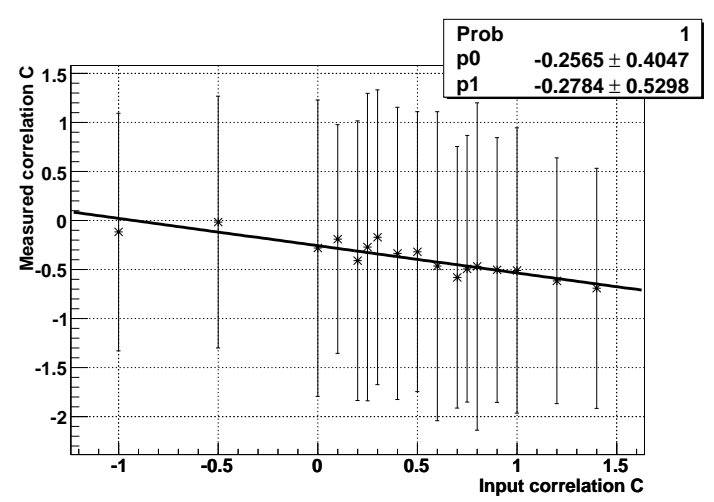

(b) Not parton - reconstructed object matching (both permutations)

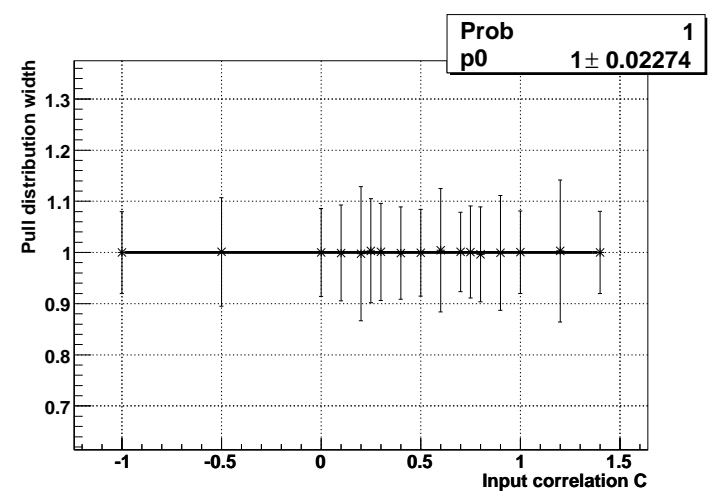

(d) Width of the pull distribution for (b)

Figure C.9.: Ensemble tests for the measurement of $C_{\bar{b} \bar{\ell}}$. 100 ensembles with 8 events each were generated. The error bars in the calibration curve plots indicate $\sigma_{C_{\text {meas }}^{\text {all ensembles }}}$ 


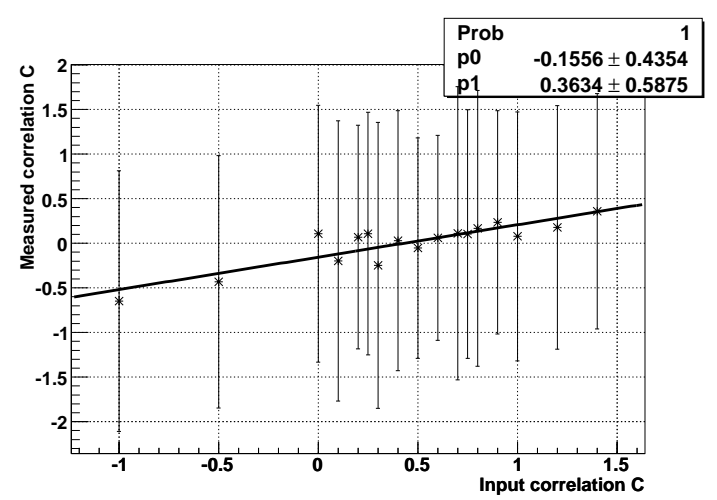

(a) t, W masses known exactly

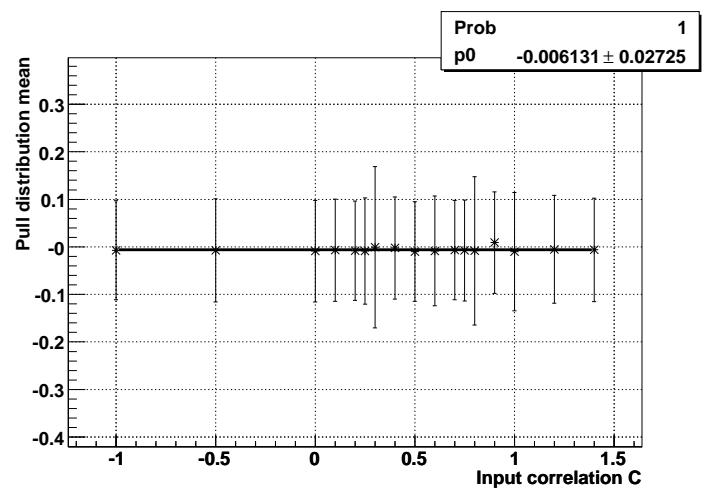

(c) Mean of the pull distribution for (b)

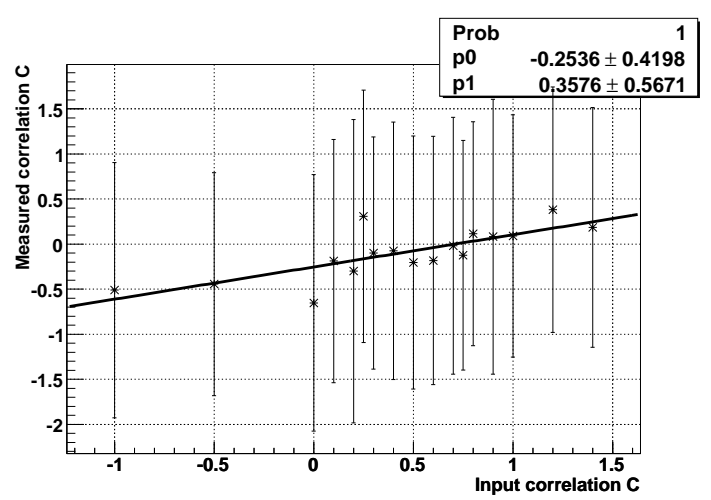

(b) Not parton - reconstructed object matching (both permutations)

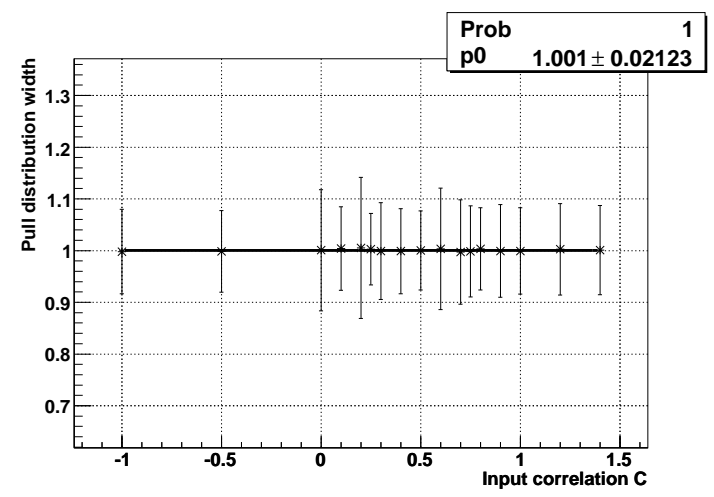

(d) Width of the pull distribution for (b)

Figure C.10.: Ensemble tests for the measurement of $C_{W^{-} W^{+}} .100$ ensembles with 8 events each were generated. The error bars in the calibration curve plots indicate $\sigma_{C_{\text {meas }}^{\text {all ensembles }}}$ 


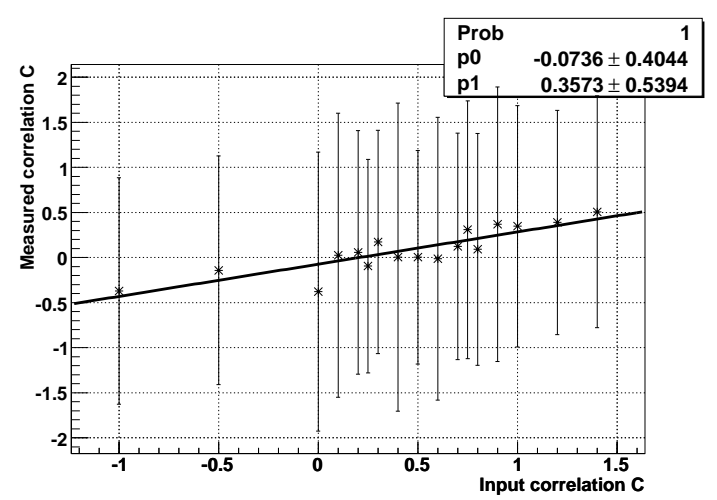

(a) t, W masses known exactly

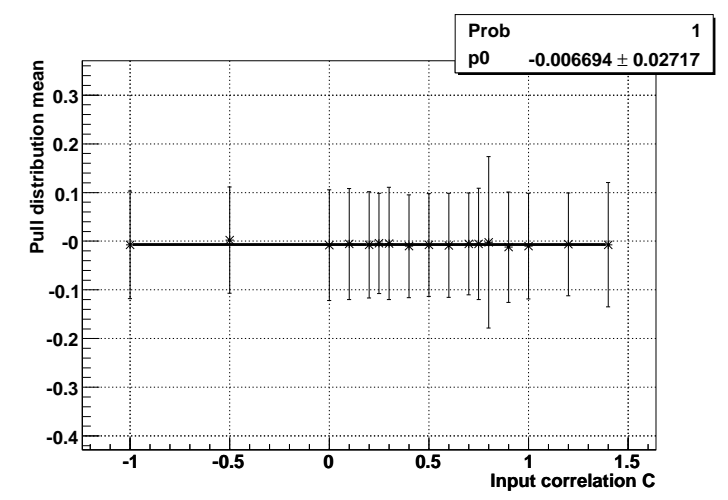

(c) Mean of the pull distribution for (b)

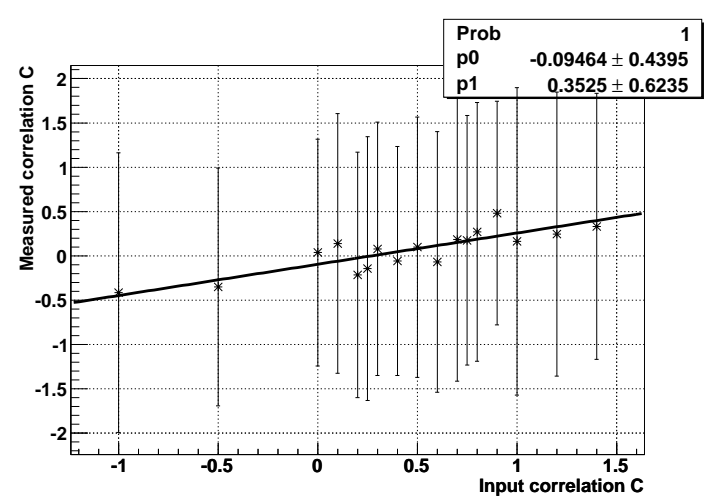

(b) Not parton - reconstructed object matching (both permutations)

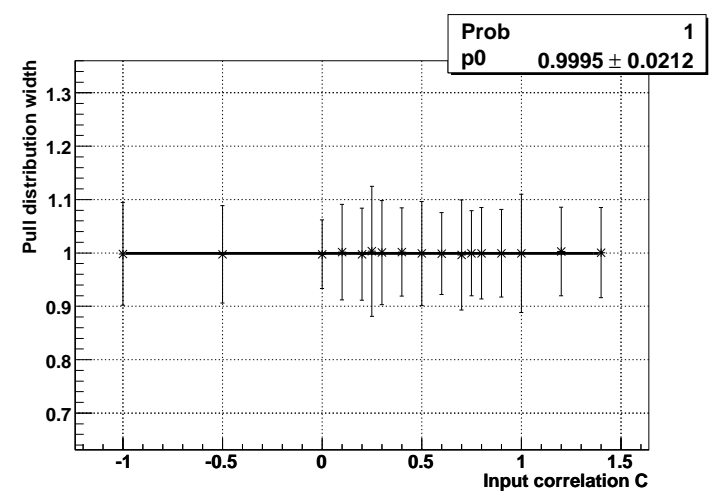

(d) Width of the pull distribution for (b)

Figure C.11.: Ensemble tests for the measurement of $C_{W-\bar{\ell} \cdot} \cdot 100$ ensembles with 8 events each were generated. The error bars in the calibration curve plots indicate $\sigma_{C_{\text {meas }}^{\text {all ensembles }}}$ 


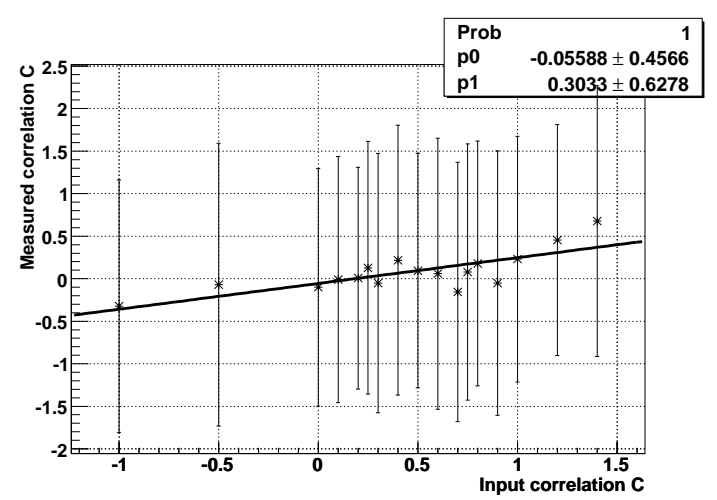

(a) t, W masses known exactly

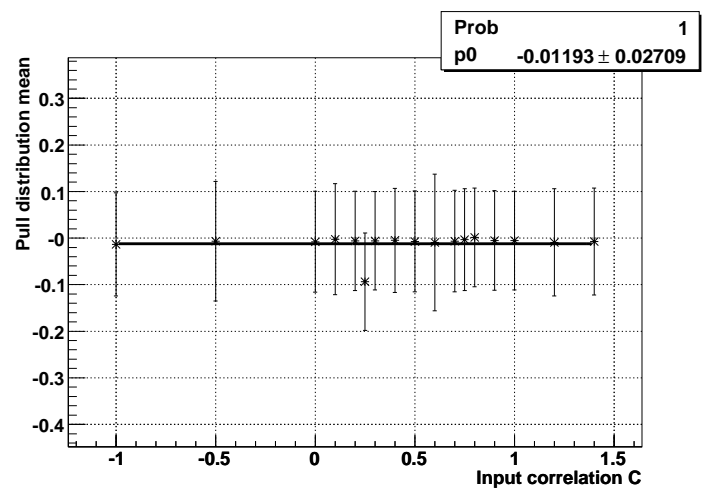

(c) Mean of the pull distribution for (b)

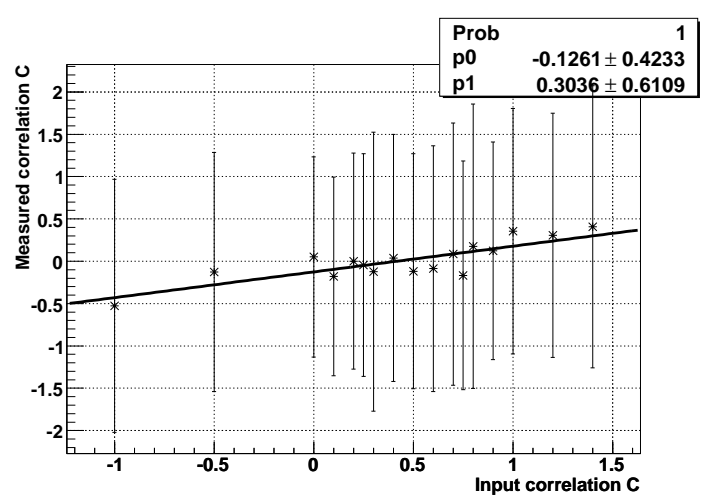

(b) Not parton - reconstructed object matching (both permutations)

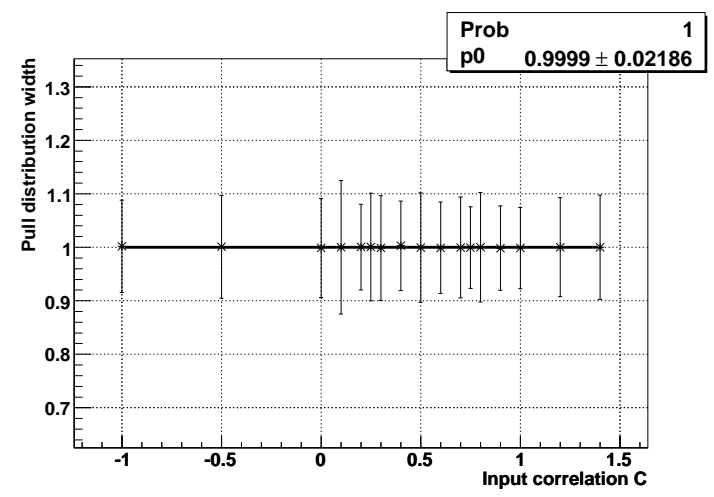

(d) Width of the pull distribution for (b)

Figure C.12.: Ensemble tests for the measurement of $C_{W^{+} \ell} .100$ ensembles with 8 events each were generated. The error bars in the calibration curve plots indicate $\sigma_{C_{\text {meas }}^{\text {all ensembles }}}$ 


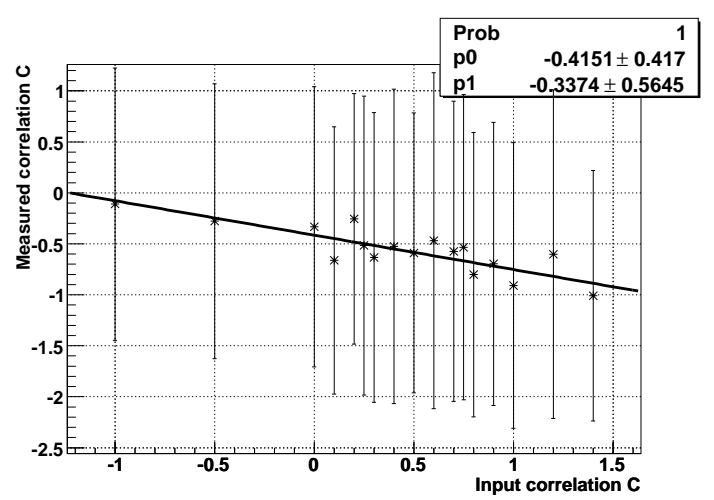

(a) t, W masses known exactly

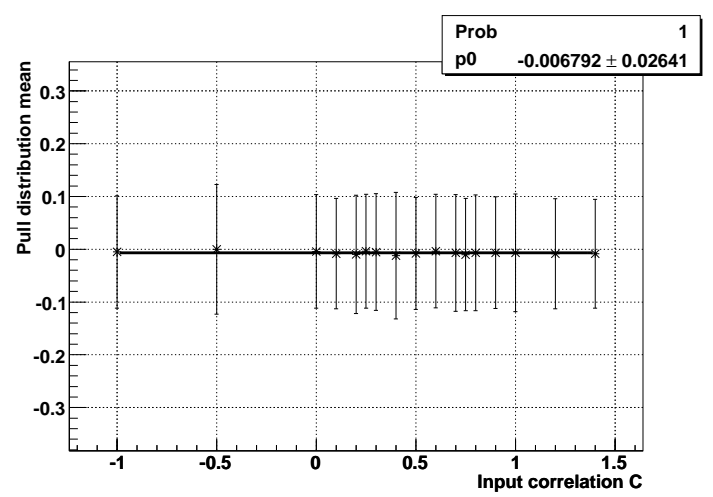

(c) Mean of the pull distribution for (b)

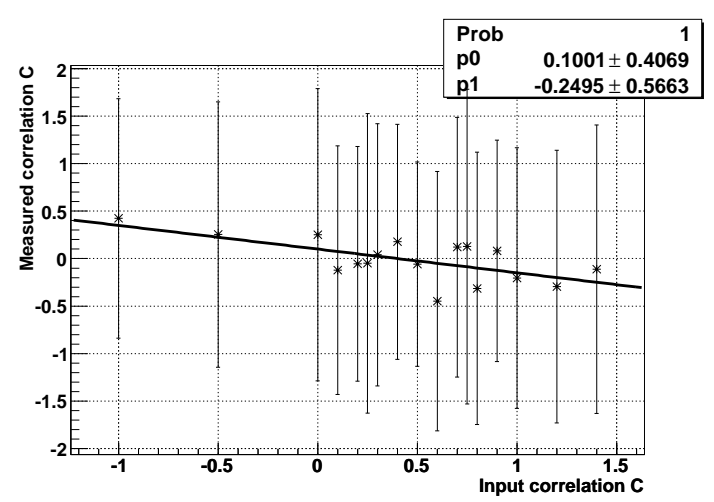

(b) Not parton - reconstructed object matching (both permutations)

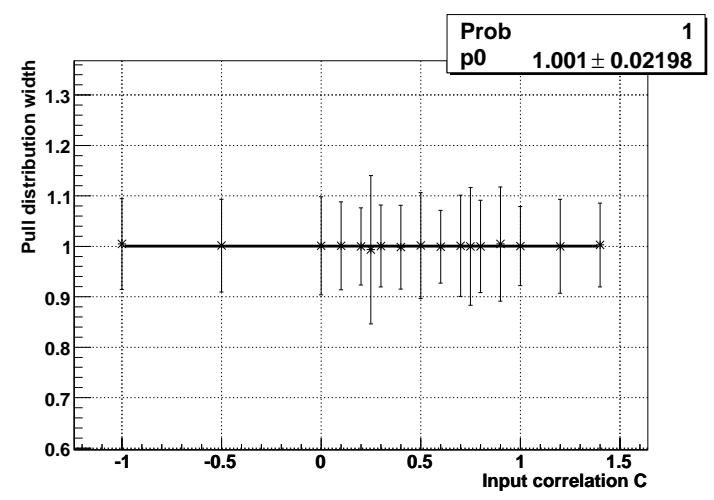

(d) Width of the pull distribution for (b)

Figure C.13.: Ensemble tests for the measurement of $C_{W^{-} b}$. 100 ensembles with 8 events each were generated. The error bars in the calibration curve plots indicate $\sigma_{C_{\text {meas }}^{\text {all ensembles }}}$ 


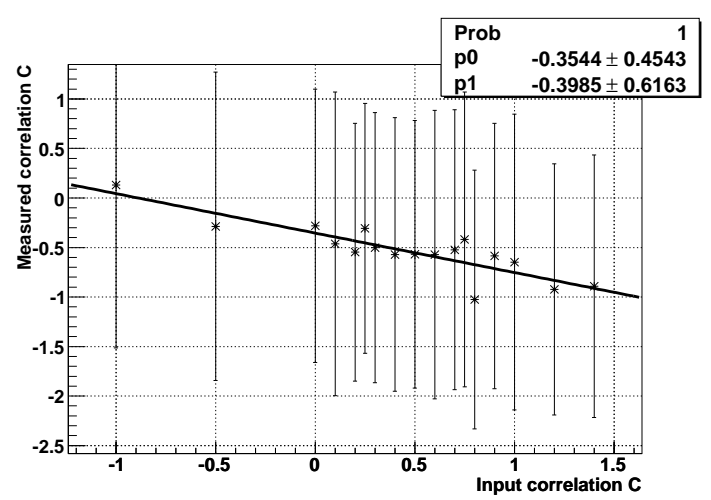

(a) t, W masses known exactly

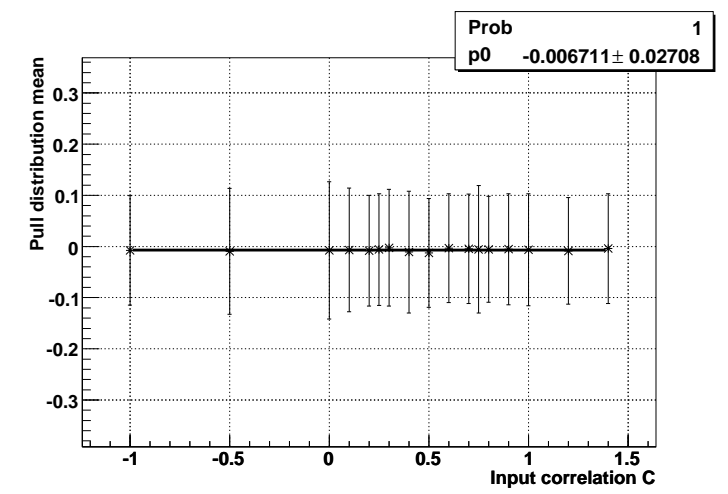

(c) Mean of the pull distribution for (b)

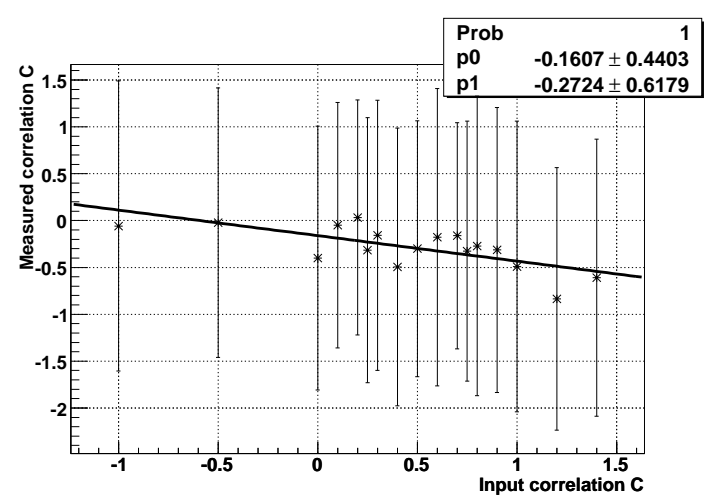

(b) Not parton - reconstructed object matching (both permutations)

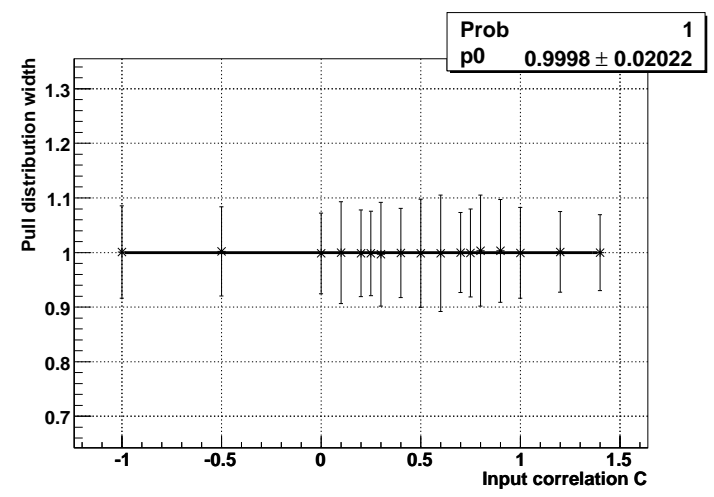

(d) Width of the pull distribution for (b)

Figure C.14.: Ensemble tests for the measurement of $C_{W+\bar{b}}$. 100 ensembles with 8 events each were generated. The error bars in the calibration curve plots indicate $\sigma_{C_{\text {meas }}^{\text {all ensembles }}}$ 
APPENDIX C. CALIBRATION PLOTS OF THE SPIN CORRELATION MEASUREMENT FOR VARIOUS INPUT SCENARIOS AND ENSEMBLE TESTS 


\section{Bibliography}

[1] U. Bassler, J. P. Konrath, C. Schwanenberger and E. Shabalina. Measurement of the $t \bar{t}$ Production Cross-section at $\sqrt{s}=1.96$ TeV in the Dimuon Final State using p17 data set. DØ Note 5350, February 2007.

[2] Y. Arnoud et al. Measurement of the $t \bar{t}$ Production Cross-Section at $\sqrt{s}=1.96$ TeV in the Dilepton Final States Using $1 \mathrm{fb}^{-1}$. DØ Note 5371-CONF, 2007.

[3] Y. Arnoud, M. Arthaud, U. Bassler, M. Besancon, S. Chakrabarti, F. Deliot, J. Konrath, B. Martin dit Latour, Y. Peters, C. Schwanenberger G. Sajot, E. Shabalina and V. Sharyy. Measurement of the Top Quark Mass using $\sigma(p \bar{p} \rightarrow t \bar{t})_{l+j e t s}$ and $\sigma(p \bar{p} \rightarrow$ $t \bar{t})_{l l}$ with the DØ Detector at $\sqrt{(s)}=1.96 \mathrm{TeV}$ in the Run II Data. DØ Note 5459, July 2007.

[4] Y. Arnoud, M. Arthaud, U. Bassler, M. Besançon, S. Chakrabarti, F. Déliot, J. Konrath, B. Martin dit Latour, G. Sajot, C. Schwanenberger, E. Shabalina, V. Sharyy, C. Tully and R. Wagner. Combined $t \bar{t}$ Production Cross Section in the Lepton+Track and Dilepton Final States using $1 \mathrm{fb}^{-1}$ of data. DØ Note 5477, 2007.

[5] U. Bassler, J. P. Konrath, C. Schwanenberger and E. Shabalina. Final Measurement

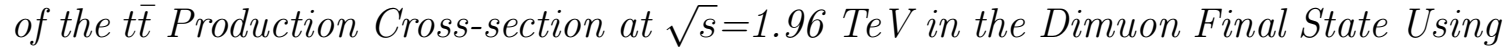
p17 Data Set. DØ Note 5581, 2008.

[6] Todd Adams, Jochen Cammin, Frederic Deliot, Amnon Harel, Jens-Peter Konrath, Bertrand Martin, Yvonne Peters, Christian Schwanenberger, Elizaveta Shabalina, Viatcheslav Sharyy, Haryo Sumowidagdo and Daniel Wicke. Combination of the Top Quark Pair Production Cross Sections and Search for Charged Higgs Boson. DØ Note 5597, February 2008.

[7] Todd Adams, Jochen Cammin, Frederic Deliot, Amnon Harel, Jens-Peter Konrath, Bertrand Martin, Yvonne Peters, Christian Schwanenberger, Elizaveta Shabalina, Viatcheslav Sharyy, Haryo Sumowidagdo and Daniel Wicke. A Search for Charged

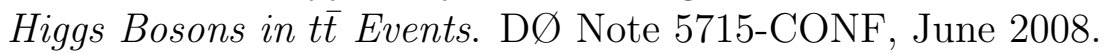

[8] David J. Griffiths, Introduction to Elementary Particles, John Wiley \& Sons New York Chichester Brisbane Toronto Singapore, 1987.

[9] F. Mandl and G. Shaw, Quantum Field Theory, John Wiley \& Sons New York Chichester Brisbane Toronto Singapore, 1993.

[10] J. Donoghue, B. Holstein and E. Golovich, Dynamics of the Standard Model, Cambridge University Press, 1994.

[11] Michael Peskin and Daniel Schroeder, An Introduction to Quantum Field Theory, Addison-Wesley Publishing Company, 1995. 
[12] Byron P. Roe, Particle Physics at the New Millennium, Springer-Verlag New York Berlin Heidelberg, 1996.

[13] Francis Halzen and Alan Martin, Quarks and Leptons. An Introductory Course in Modern Particle Physics, Wiley/VCH, 1998.

[14] Steven Weinberg, The Quantum Theory of Fields 1: Foundations, Cambridge University Press, 2005.

[15] Steven Weinberg, The Quantum Theory of Fields 2: Modern Applications, Cambridge University Press, 2005.

[16] W.N. Cottingham and D.A. Greenwood, An Introduction to the Standard Model of Particle Physics, Cambridge University Press, 2007.

[17] Brian R. Martin and Graham P. Shaw, Particle Physics, John Wiley and Sons Ltd, 2008.

[18] W.-M. Yao, C. Amsler, D. Asner, R.M. Barnett, J. Beringer, P.R. Burchat, C.D. Carone, C. Caso, O. Dahl, G. D'Ambrosio, A. DeGouvea, M. Doser, S. Eidelman, J.L. Feng, T. Gherghetta, M. Goodman, C. Grab, D.E. Groom, A. Gurtu, K. Hagiwara, K.G. Hayes, J.J. Hernández-Rey, K. Hikasa, H. Jawahery, C. Kolda, Kwon Y., M.L. Mangano, A.V. Manohar, A. Masoni, R. Miquel, K. Mönig, H. Murayama, K. Nakamura, S. Navas, K.A. Olive, L. Pape, C. Patrignani, A. Piepke, G. Punzi, G. Raffelt, J.G. Smith, M. Tanabashi, J. Terning, N.A. Törnqvist, T.G. Trippe, P. Vogel, T. Watari, C.G. Wohl, R.L. Workman, P.A. Zyla, B. Armstrong, G. Harper, V.S. Lugovsky, P. Schaffner, M. Artuso, K.S. Babu, H.R. Band, E. Barberio, M. Battaglia, H. Bichsel, O. Biebel, P. Bloch, E. Blucher, R.N. Cahn, D. Casper, A. Cattai, A. Ceccucci, D. Chakraborty, R.S. Chivukula, G. Cowan, T. Damour, T. DeGrand, K. Desler, M.A. Dobbs, M. Drees, A. Edwards, D.A. Edwards, V.D. Elvira, J. Erler, V.V. Ezhela, W. Fetscher, B.D. Fields, B. Foster, D. Froidevaux, T.K. Gaisser, L. Garren, H.-J. Gerber, G. Gerbier, L. Gibbons, F.J. Gilman, G.F. Giudice, A.V. Gritsan, M. Grünewald, H.E. Haber, C. Hagmann, I. Hinchliffe, A. Höcker, P. Igo-Kemenes, J.D. Jackson, K.F. Johnson, D. Karlen, B. Kayser, D. Kirkby, S.R. Klein, K. Kleinknecht, I.G. Knowles, R.V. Kowalewski, P. Kreitz, B. Krusche, Yu.V. Kuyanov, O. Lahav, P. Langacker, A. Liddle, Z. Ligeti, T.M. Liss, L. Littenberg, L. Liu, K.S. Lugovsky, S.B. Lugovsky, T. Mannel, D.M. Manley, W.J. Marciano, A.D. Martin, D. Milstead, M. Narain, P. Nason, Y. Nir, J.A. Peacock, S.A. Prell, A. Quadt, S. Raby, B.N. Ratcliff, E.A. Razuvaev, B. Renk, P. Richardson, S. Roesler, G. Rolandi, M.T. Ronan, L.J. Rosenberg, C.T. Sachrajda, S. Sarkar, M. Schmitt, O. Schneider, D. Scott, T. Sjöstrand, G.F. Smoot, P. Sokolsky, S. Spanier, H. Spieler, A. Stahl, T. Stanev, R.E. Streitmatter, T. Sumiyoshi, N.P. Tkachenko, G.H. Trilling, G. Valencia, K. van Bibber, M.G. Vincter, D.R. Ward, B.R. Webber, J.D. Wells, M. Whalley, L. Wolfenstein, J. Womersley, C.L. Woody, A. Yamamoto, O.V. Zenin, J. Zhang and R.-Y. Zhu, Review of Particle Physics, Journal of Physics G 33 (2006) 1+.

[19] S. L. Glashow, Nucl. Phys. 22 (1961) 579.

[20] S. Weinberg, Phys. Rev. Lett. 19 (1967) 1264. 
[21] A. Salam. In N. Svartholm, editor, Elementary Particle Theory. Almquist and Wiksell, 1968.

[22] J. Pumplin, D. R. Stump, J. Huston, H. L. Lai, P. Nadolsky and W. K. Tung, New Generation of Parton Distributions with Uncertainties from Global QCD Analysis, JHEP 0207 (2002) 012.

[23] Daniel Stump, Joey Huston, Jon Pumplin, Wu-Ki Tung, H. L. Lai, Steve Kuhlmann and J. F. Owens, Inclusive Jet Production, Parton Distributions, and the Search for New Physics, JHEP 0310 (2003) 046.

[24] J. Pumplin, A. Belyaev, J. Huston, D. Stump and W. K. Tung, Parton Distributions and the Strong Coupling Strength: CTEQ6AB PDFs, JHEP 0602 (2006) 032.

[25] W. K. Tung, H. L. Lai, A. Belyaev, J. Pumplin, D. Stump and C. P. Yuan, Heavy Quark Mass Effects in Deep Inelastic Scattering and Global QCD Analysis, JHEP 0702 (2007) 053.

[26] J. Pumplin, H. L. Lai and W. K. Tung, The Charm Parton Content of the Nucleon (2007).

[27] H. L. Lai, P. Nadolsky, J. Pumplin, D. Stump, W. K. Tung and C. P. Yuan, The Strange Parton Distribution of the Nucleon: Global Analysis and Applications (2007).

[28] A. D. Martin, R. G. Roberts, W. J. Stirling and R. S. Thorne, Uncertainties of Predictions from Parton Distributions. I: Experimental Errors, Eur.Phys.J. C 28 (2003) 455-473.

[29] A. D. Martin, R. G. Roberts, W. J. Stirling and R. S. Thorne, Physical Gluons and High $E_{T}$ Jets, Phys.Lett. B 604 (2004) 61-68.

[30] A. D. Martin, R. G. Roberts, W. J. Stirling and R. S. Thorne, Parton Distributions Incorporating QED Contributions, Eur.Phys.J. C 39 (2005) 155-161.

[31] A. D. Martin, W. J. Stirling and R. S. Thorne, MRST Partons Generated in a FixedFlavour Scheme, Phys.Lett. B 636 (2006) 259-264.

[32] R. S. Thorne, A Variable-Flavour Number Scheme for NNLO, Phys.Rev. D 73 (2006) 054019 .

[33] A. D. Martin, W. J. Stirling, R. S. Thorne and G. Watt, Update of Parton Distributions at NNLO, Phys.Lett. B 652 (2007) 292-299.

[34] Wolfgang Wagner, Top Quark Physics in Hadron Collisions, Rept.Prog.Phys. 68 (2005) 2409-2494.

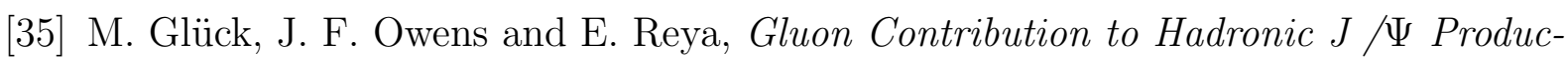
tion, Phys. Rev. D 17 (1978) 2324.

[36] John Babcock, Dennis Sivers and Stephen Wolfram, Quantum-Chromodynamic Estimates for Heavy-Particle Production, Phys. Rev. D 18 (1978) 162. 
[37] Kaoru Hagiwara and T. Yoshino, Hadroproduction of Heavy Quark Flavors in QCD, Phys.Lett. B 80 (1978) 282.

[38] P. Nason, S. Dawson and R. K. Ellis, The Total Cross Section for the Production of Heavy Quarks in Hadronic Collisions, Nucl. Phys. B 303 (1988) 607.

[39] Nikolaos Kidonakis and George Sterman, Resummation for QCD Hard Scattering, Nucl. Phys. B505 (1997) 321-348.

[40] R. Bonciani, S. Catani, M. L. Mangano and P. Nason, NLL Resummation of the Heavy-Quark Hadroproduction Cross-section, Nucl.Phys. B 529 (1998) 424-450.

[41] Nikolaos Kidonakis, Eric Laenen, Sven Moch and Ramona Vogt, Sudakov Resummation and Finite Order Expansions of Heavy Quark Hadroproduction Cross Sections, Phys. Rev. D64 (2001) 114001.

[42] Nikolaos Kidonakis and Ramona Vogt, Next-to-Next-to-Leading Order Soft-Gluon Corrections in Top Quark Hadroproduction, Phys.Rev. D 68 (2003) 114014.

[43] Sven Moch and Peter Uwer, Theoretical Status and Prospects for Top-Quark Pair Production at Hadron Colliders (2008).

[44] Nikolaos Kidonakis and Ramona Vogt, Theoretical Status of the Top Quark Cross Section, Int.J.Mod.Phys. A 20 (2005) 3171.

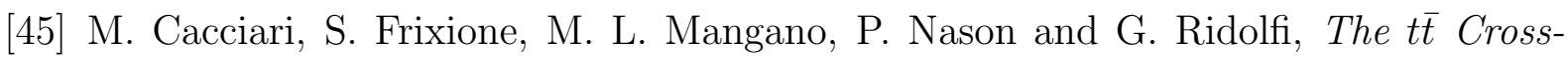
Section at 1.8 and $1.96 \mathrm{TeV}$ : A Study of the Systematics Due to Parton Densities and Scale Dependence, JHEP 0404 (2004) 068.

[46] Matteo Cacciari, Stefano Frixione, Michelangelo M. Mangano, Paolo Nason and Giovanni Ridolfi, Updated Predictions for the Total Production Cross Sections of Top and of Heavier Quark Pairs at the Tevatron and at the LHC (2008).

[47] S. Moch and P. Uwer, Heavy-Quark Pair Production at two Loops in QCD (2008).

[48] W. Bernreuther, A. Brandenburg, Z. G. Si and P. Uwer, Top Quark Pair Production and Decay at Hadron Colliders, Nucl.Phys. B 690 (2004) 81-137.

[49] T. Stelzer and S. Willenbrock, Spin Correlation in Top-Quark Production at Hadron Colliders, Phys.Lett. B 374 (1996) 169-172.

[50] G. L. Kane, G. A. Ladinsky and C.-P. Yuan, Using the Top Quark for Testing Standard-Model Polaraization and CP Predictions, Phys.Rev. D 45 (1992) 124-141.

[51] Gregory Mahlon and Stephen Parke, Angular Correlations in Top Quark Pair Production and Decay at Hadron Colliders, Phys.Rev. D 53 (1996) 4886-4896.

[52] Gregory Mahlon and Stephen Parke, Improved Spin Basis for Angular Correlation Studies in Single Top Quark Production at the Tevatron, Phys.Rev. D 55 (1997) 7249-7254.

[53] Brent Evanger, Cons Gattuso, Andrew Braun, Denton Morris, Robin Spayde and Bruce Worthel. Accelerator Concepts Rookie Book, Version 3, 2002. 
[54] Evanger. The Tavatron Rookie Book.

[55] D0 Collaboration, The Upgraded D0 Detector, Nucl.Instrum.Meth. A 565 (2006) 463-537.

[56] Alexander Khanov. HTF: Histogramming Method for Finding Tracks. The Algorithm Description. DØ Note 3778, September 2000.

[57] G. Borissov. Ordering a Chaos or... Technical Details of AA Tracking. http://wwwd0.fnal.gov/atwork/adm/d0_private/2003-02-28/adm_talk.ps, 2003.

[58] Ariel Schwartzman and Chris Tully. Primary Vertex Reconstruction by Means of Adaptive Vertex Fitting. DØ Note 4918, September 2005.

[59] Philippe Calfayan, Thomas Gadfort, Gavin Hesketh, Vincent Lesne, Mark Owen, Raimund Stroehmer, Viatcheslav Sharyy and Boris Tuchming. Muon Identification Certification for p17 data. DØ Note 5157, June 2006.

[60] http://www-d0.fnal.gov/computing/algorithms/muon/p17/muonId_quality_type.html.

[61] Laurent Duflot, Guy Le Meur and Francois Touze. The ConeClusterAlgo User Guide. http://www-d0.fnal.gov/d0dist/dist/releases/p17.09.06/calreco/doc/ConeAlgo.ps, June 1998.

[62] Serban Protopopescu. EMReco Algorithms. http://wwwd0.fnal.gov/d0dist/dist/releases/p17.09.06/emreco/doc/EMReco.ps, June 1999.

[63] R. A. Fisher, Annals of Eugenics 7 (1936) 179.

[64] P. C. Mahalanobis, On the Generalized Distance in Statistics, Proceedings of the National Institute of Science of India 12 (1936) 49.

[65] E. Busato and B. Andrieu. Jet Algorithms in D0 RunII Software: Description and User's Guide. DØ Note 4457, May 2004.

[66] Bernard Andrieu. Jet Finding Algorithms at Tevatron. DØ Note 5168, July 2006.

[67] Amnon Harel. Jet ID Optimization. DØ Note 4919, September 2005.

[68] DØ Jet Energy Scale Group. Jet Energy Scale Determination at DØ Run II (final p17 version). DØ Note 5382, 2007.

[69] M. L. Mangano, M. Moretti and R. Pittau, Multijet Matrix Elements and Shower Evolution in Hadronic Collisions: $W b \bar{b}+n$ jets as a Case Study, Nucl. Phys. B 632 (2002) 343.

[70] M. L. Mangano, M. Moretti, F. Piccinini, R. Pittau and A. D. Polosa, ALPGEN, a Generator for Hard Multiparton Processes in Hadronic Collisions, JHEP 0307 (2003) 001.

[71] F. Caravaglios, M. L. Mangano, , M. Moretti and R. Pittau, A New Approach to Multijet Calculations in Hadron Collisions, Nucl. Phys. B 539 (1999) 215. 
[72] Torbjörn Sjöstrand, Leif Lönnblad, Stephen Mrenna and Peter Skands, High Physics Event Generation with PYTHIA 6.1, Comput. Phys. Comm. 135 (2001) 238.

[73] Torbjörn Sjöstrand, Leif Lönnblad, Stephen Mrenna and Peter Skands, PYTHIA 6.3 Physics and Manual (2003).

[74] M. R. Whalley, D. Bourilkov and R. C. Group, The Les Houches Accord PDFs (LHAPDF) and Lhaglue (2007).

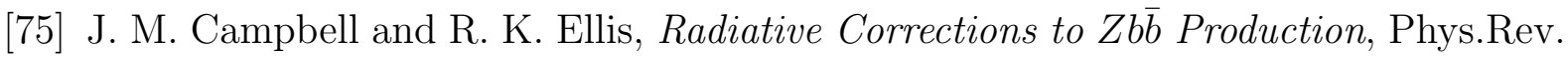
D 62 (2000) 114012.

[76] Thomas Nunnemann. NNLO Cross-Sections for Drell-Yan, $Z$ and $W$ Production using Modern Parton Distribution Functions. DØ Note 4476, 2004.

[77] Stefan Höche, Frank Krauss, Nils Lavesson, Leif Lönnblad, Michelangelo Mangano, Andreas Schälicke and Steffen Schumann, Matching Parton Showers and Matrix Elements (2006).

[78] Guido Altarelli and G. Parisi, Asymptotic Freedom in Parton Language, Nucl. Phys. B126 (1977) 298.

[79] R. Brun and F. Carminati. CERN Program Library Long Writeup. W5013, 1993.

[80] Heidi Schellman. The Longitudinal Shape of the Luminous Region at DØ. 5142.

[81] Avdhesh Chandra and Juan Estrada. Beam Width Measurement at DØ. DØ Note 4753 .

[82] Philippe Calfayan. ORing Single Muon Triggers in p17 Data. DØ Note 5329, January 2007.

[83] Nikola Makovec and Jean-Francois Grivaz. Shifting, Smearing and Removing Simulated Jets. DØ Note 4914, November 2005.

[84] Z. Was and P. Golonka, TAUOLA as $\tau$ Monte Carlo for Future Applications (2007).

[85] Dag Gillberg. Heavy Flavour Removal and Determination of Weighting Factors for ALPGEN W+jet Monte Carlo. DØ Note 5129, May 2006.

[86] J.D. Hobbs, T. Nunnemann and R. Van Kooten. Study of $p \bar{p} \rightarrow Z / \gamma^{*} \rightarrow e e, \mu \bar{\mu}$ Event Yields as a Luminosity Cross Check. DØ Note 5268, October 2006.

[87] T. Nunnemann. MCFM Cross Sections.

[88] Bertrand Martin. p17 Alpgen Z $p_{T}$ Reweighting. DØ Note 5571, January 2008.

[89] Shaohua Fu and Andy Haas. Search for ZH-illbb (p17 with NN). DØ Note 5482CONF, August 2007.

[90] Ariel Schwartzman. $E_{T}$ Significance Algorithm in RunII Data. DØ Note 4254, September 2003. 
[91] Bruce Knuteson, Mark Strovink, Bob Olivier, Ursula Bassler, Frederic Fleuret and Gregorio Bernardi. $E_{T}$ : The Missing Transverse Energy Resolution of an Event. DØ Note 3629, June 2000.

[92] Laurent Duflot, Viatcheslav Shary, Ingo Torchiani and Robert Zitoun. cal_event_quality Package. DØ Note 4614, September 2004.

[93] Su-Jung Park and Michael Begel. Efficiency of the Data Quality Calorimeter Flags. DØ Note 5324, January 2007.

[94] S. Anderson, S. Burke, B. Choudhary, C. Clement, R. Kehoe, J. Kozminski, A. Kumar, J. Leveque, K. Ranjan and E. Shabalina. Measurement of the $t \bar{t}$ Production Cross Section at $\sqrt{s}=1.96 \mathrm{TeV}$ in ee and $\mu \mu$ Final States Using $370 \mathrm{pb}^{-1}$ of Pass 2 Data. DØ Note 4827, May 2005.

[95] E. Aguilo, P. Baringer, A. Bean, C. Belanger-Champagne, J.A. Benitez, E.E. Boos, R. Brock, V. Bunichev, K. Chan, L. Christofek, Y. Coadou, L.V. Dudko, M. Erdmann, T. Gadfort, A. Garcia-Bellido, C. Gerber, D. Gillberg, G. Gutierrez, P. Gutierrez, A.P. Heinson, U. Heintz, S. Herrin, S. Jabeen, S. Jain, A. Juste, S. Kappler, D. Kau, G. Kertzscher, M. Kirsch, L. Li, J. Mitrevski, R. Moore, M. Narain, D. O’Neil, M. Pangilinan, J. Parsons, M. Perfilov, C. Potter, H.B. Prosper, R. Schwienhorst, E. Shabalina, J. Steggemann, T. Tim, C. Tully, M. Vetterli, B. Vachon, G. Watts and M. Weber. Search for Single Top Quark Production in 1 fb-1 of Data. DØ Note, November 2006.

[96] M. Arthaud, M. Besancon, S. Chakrabarti, F. Deliot and V. Sharyy. Final Measurement of the $t \bar{t}$ Production Cross-Section at $\sqrt{s}=1.96$ TeV in Electron Muon Final States Ssing p17 Data Set. DØ Note 5580, February 2008.

[97] B. Martin, Y. Arnoud, G. Sajot and E. Shabalina. Final Measurement of the $t \bar{t}$ Production Cross Section at $\sqrt{s}=1.96$ TeV in the ee Final State Using p17 Data Set. DØ Note 5579, 2008.

[98] O. Brandt, R. Kehoe, J. Meyer, A. Quadt, P. Renkel and C. Schwanenberger. Measurement of the Top Quark Mass in Dilepton Events with Neutrino Weighting. DØ Note 5346, February 2007.

[99] Daniel Boline and Ulrich Heintz. Measurement of the Top Quark Mass in the Dilepton Channel Using the Matrix Weighting Method at DØ. DØ Note 5462, July 2007.

[100] The Tevatron Electroweak Working Group, for the CDF and D0 Collaborations, Combination of CDF and DO Results on the Mass of the Top Quark (2008).

[101] S. Chevalier-Thery, U. Bassler, M. Cacciari, F. Deliot, U. Heintz, Y. Peters and C. Schwanenberger. Top Quark Mass Extraction from $t \bar{t}$ Cross Section Measurements. DØ Note 5742, July 2008.

[102] S. P. Martin, A Supersymmetry Primer (1999). hep-ph/9709356v4. 
[103] J. S. Lee, A. Pilaftsis, M. Carena, S. Y. Choi, M. Drees, J. Ellis and C. E. M. Wagner, CPsuperH: a Computational Tool for Higgs Phenomenology in the Minimal Supersymmetric Standard Model with Explicit CP Violation, Comput.Phys.Commun. 156 (2004) 283-317.

[104] Gary Feldman and Robert Cousins, Unified Approach to the Classical Statistical Analysis of Small Signals, Phys.Rev. D 57 (1998) 3873-3889.

[105] Marcel Demarteau, Pieter Houben, Marcel Vreeswijk and Michele Weber. p17 Parton Level Corrections and Resolutions for Hitfit. DØ Note 5418, June 2007.

[106] Sarosh N. Fatakia and Ulrich Heintz. Correcting the Reconstructed Jet's 4-Vector to the Parton Level. DØ Note 4247, September 2003.

[107] Lars Sonnenschein, Analytical Solution of $t \bar{t}$ Dilepton Equations, Phys.Rev. D 73 (2006) 054015. 